\title{
A prospective cohort study on diet and cancer in the Netherlands : design, conduct, analysis and first results after 3.3 years of follow-up
}

Citation for published version (APA):

van den Brandt, P. A. (1993). A prospective cohort study on diet and cancer in the Netherlands : design, conduct, analysis and first results after 3.3 years of follow-up. [Doctoral Thesis, Maastricht University]. Datawyse / Universitaire Pers Maastricht. https://doi.org/10.26481/dis.19930128pb

Document status and date:

Published: 01/01/1993

DOI:

10.26481/dis.19930128pb

Document Version:

Publisher's PDF, also known as Version of record

Please check the document version of this publication:

- A submitted manuscript is the version of the article upon submission and before peer-review. There can be important differences between the submitted version and the official published version of record.

People interested in the research are advised to contact the author for the final version of the publication, or visit the DOI to the publisher's website.

- The final author version and the galley proof are versions of the publication after peer review.

- The final published version features the final layout of the paper including the volume, issue and page numbers.

Link to publication

\footnotetext{
General rights rights.

- You may freely distribute the URL identifying the publication in the public portal. please follow below link for the End User Agreement:

www.umlib.nl/taverne-license

Take down policy

If you believe that this document breaches copyright please contact us at:

repository@maastrichtuniversity.nl

providing details and we will investigate your claim.
}

Copyright and moral rights for the publications made accessible in the public portal are retained by the authors and/or other copyright owners and it is a condition of accessing publications that users recognise and abide by the legal requirements associated with these

- Users may download and print one copy of any publication from the public portal for the purpose of private study or research.

- You may not further distribute the material or use it for any profit-making activity or commercial gain

If the publication is distributed under the terms of Article $25 \mathrm{fa}$ of the Dutch Copyright Act, indicated by the "Taverne" license above, 


\section{A PROSPECTIVE COHORT STUDY ON DIET AND CANCER IN THE NETHERLANDS}

DESIGN, CONDUCT, ANALYSIS AND

FIRST RESULTS AFTER 3.3 YEARS OF FOLLOW-UP 


\title{
A PROSPECTIVE COHORT STUDY ON DIET AND CANCER IN THE NETHERLANDS
}

\author{
DESIGN, CONDUCT, ANALYSIS AND \\ FIRST RESULTS AFTER 3.3 YEARS OF FOLLOW-UP
}

\section{PROEFSCHRIFT}

ter verkrijging van de graad van doctor aan de Rijksuniversiteit Limburg te Maastricht, op gezag van de Rector Magnificus, Prof. Mr. M.J. Cohen, volgens het besluit van het College van Decanen, in het openbaar te verdedigen op donderdag, 28 januari 1993 om 14.00 uur

door

Petrus Adrianus van den Brandt

en

Royle Alexandra Bausch-Goldbohm 
Promotores:

Beoordelingscommissie:
Prof. dr. F. Sturmans

Prof. dr. R.J.J. Hermus

Prof. dr. W.H.M. Saris, RL (voorzitter)

Prof. dr. J.A. Knottnerus, RL

Prof. dr. R. Kroes, RUU

Prof. dr. P.B. Soeters, RL

Prof. dr. W.C. Willett, Harvard University

\section{CIP-GEGEVENS KONINKL.IJKE BIBLIOTHEEK, DEN HAAG}

Brandt, Petrus Adrianus van den

A prospective cohort study on diet and cancer in the Netherlands : design, conduct, analysis and first results after 3.3 years of follow-up / Petrus Adrianus van den Brand, Royle Alexandra Bausch-Goldbohm. - Maastricht :

Universitaire Pers Maastricht. - Ill.

Thesis Maastricht. - With ref.

ISBN 90-5278-057-9

NUGI $741 / 755$

Subject headings: diet / neoplasms / epidemiology.

Lay-out: Thum Aarts, Epidemiologie RL, Maastricht Produktie: Datawyse | Universitaire Pers Maastricht

Het onderzoek waarop dit proefschrift is gebaseerd werd verricht vanuit de vakgroep Epidemiologie van de Rijksuniversiteit Limburg (hoofd: Prof. Dr. P. Knipschild) in Maastricht en de afdeling Voeding van het Instituut Toxicologie en Voeding TNO (hoofd: Dr. Th. Ockhuizen) in Zeist, met financiële steun van de Nederlandse Kankerbestrijding, de Europese Gemeenschap, het Ministerie van Welzijn, Volksgezondheid en Cultuur en het Produktischap Vee en Vlees.

In de drukkosten van dit proefschrift werd bijgedragen door de Nederlandse Kankerbestrijding, Stichting Dr. Ir. J.H.J. van de Laar, Pharmachemie B.V. Haarlem, Janssen Pharmaceutica B.V., Glaxo B.V., Nutricia B.V.. 


\section{Contents}

\section{PART I: Design and follow-up}

2 A large-scale prospective cohort study on diet and cancer in the Netherlands

3 Estimation of the coverage of municipalities by cancer registries and PALGA using hospital discharge data

4 Development of a record linkage protocol for use in the Dutch cancer registry for epidemiological research

\section{PART II: Exposure assessment}

5 Validation of a dietary questionnaire used in a large-scale prospective cohort study on diet and cancer

6 Reproducibility of a food frequency questionnaire and stability of dietary habits determined from five annually repeated measurements

7 Predictors of toenail selenium levels in men and women

8 Assessment of dietary nitrate intake by a self-administered questionnaire and by overnight urinary measurement

9 Cross-sectional versus longitudinal investigations of the diet-cancer relation

PART III: Statistical analysis

10 Stratified and simple regression methods for the analysis of case-cohort studies 


\section{PART IV: Results}

11 A prospective cohort study on dietary fat and the risk of postmenopausal breast cancer

12 Prospective study on alcohol consumption and the risk of cancer of the colon and rectum

13 A prospective cohort study on the relation between meat consumption and the risk of colon cancer

14 Cholecystectomy and colorectal cancer:

evidence from a cohort study on diet and cancer

15 A prospective cohort study on selenium status and the risk of lung cancer

16 A prospective cohort study on toenail selenium and the risk of gastrointestinal cancer

17 Toenail selenium and the subsequent risk of breast cancer

18 Epllogue

Summary

Samenvatting

Dankwoord

Appendix: Self-administered questionnaire

About the authors 
The following table shows which author is primarily responsible for each chapter.

Author primarily responsible

\begin{tabular}{lll} 
Chapter & P.A. van den Brandt & R.A. Bausch-Goldbohm \\
\hline 1 & X & X \\
2 & X & X \\
3 & & X \\
4 & X & \\
5 & & X \\
6 & & $X$ \\
7 & $X$ & \\
8 & $X$ & \\
9 & $X$ & \\
10 & $X$ & \\
11 & $X$ & $X$ \\
12 & & $X$ \\
13 & & $X$ \\
14 & $X$ & \\
15 & $X$ & \\
16 & $X$ & \\
17 & $X$ & \\
18 & $X$ & \\
\hline
\end{tabular}




\section{Chapter 1}

\section{Introduction}

\section{Background}

In the early 1980s the Dutch Cancer Society decided to stimulate the development of expertise in epidemiology, in particular cancer epidemiology, which was at that time pursued by a few scientists only. The Society put this decision into practice by taking the two following measures: (a) two-year fellowships were awarded to post-graduate scientists in order to receive a training in epidemiology and (b) epidemiological research projects were ranked high in priority for funding by the Society. Without this policy of the Dutch Cancer Society there would not have been a "Dutch prospective cohort study on diet and cancer".

The three epidemiologists who initiated the cohort study (P.A.v.d.B., R.A.B. together with Pieter van 't Veer of the TNO-Toxicology and Nutrition Institute) had many things in common: all had graduated in human nutrition, were very interested in epidemiology, were awarded an epidemiology fellowship by the Dutch Cancer Society, went to the Harvard School of Public Health (Boston, USA) for a formal training in epidemiology, and, last but not least, were supposed to write each a grant proposal for a cancer epidemiology project during the second year of their respective fellowships. Not surprisingly, the potential objectives of the grant proposals concerned the hypothesized (1) relation between dietary habits and specific tumors, to be investigated in separate case-control studies. It soon became clear, however, that here was the opportunity to combine efforts and write a joint grant proposal for a prospective colhort study in which the association of dietary habits with various types of cancer could be investigated simultaneously. Although prospective studies are also preferred to casecontrol studies in order to avoid biases - in particular information (recall) bias, which hampers inference from dietary case-control studies -, only very few of such studies on diet and cancer were being conducted at that time. The scarcity of prospective cohort studies is caused by the logistic and financial constraints resulting from the large size required for these studies and by the long follow-up period. The ongoing large-scale prospective studies on diet and cancer included five American studies: the Adventist Health Study (34,000 men and women) (2), the New York State Cohort $(58,000$ men and women) (3), the Nurses' Health Study (98,000 women) (4), the Canadian Breast Cancer Screening Study (57,000 women) (5), the Cancer Prevention Study II $(1,200,000$ men and women) (6) and one Japanese Study (265,000 men and women) (7). The Japanese study and the Cancer Prevention Study have used very brief questionnaires for the assessment of dietary habits at baseline, whereas the other studies have used more extensive methods. A prospective study in the Netherlands among a population different from that in the other studies, with different lifestyle and dietary habits, assessed by a more detailed instrument, would contribute substantially to the interpretation of the results originating from epidemiological diet and cancer studies.

\section{Scope}

This thesis describes the methodological and feasibility aspccts of the prospective cohort study on diet and cancer that we have designed and subsequently carried out. The purpose of this study is to test various existing hypotheses in the field of diet and cancer. Our initial interest is in cancers of the stomach, colon, rectum, brcast and lung 
because of their suspected relationship with diet and because of their relatively high incidence. The primary goal of the study is to investigate the associations between fats, vitamins (A, C, carotene), fiber, alcohol, selenium, nitrate, sodium and calcium on the development of gastric, colorectal, breast and lung tumors $(8,9)$. Besides nutrients we are also interested in associations with particular foods (e.g., meat, alcoholic beverages) and dietary patterns. While the scope of this thesis is to describe how the study was designed and conducted, we also present results on the first etiological analyses to illustrate what can and what cannot (yet) be done with the data accumulating from the study. For this purpose, we decided to test some prominent hypotheses. These relate to the intake of fat and meat and risk of breast and colorectal cancer, and to alcohol intake and colorectal cancer risk. An illustration of how the cohort study can be used to investigate whether detection or ascertainment bias can explain particular associations found in case-control studies is presented with the analysis of cholecystectomy and colorectal cancer. Finally, we present results regarding selenium status and the risk of lung. gastrointestinal and breast cancer. This is to illustrate a distinct advantage of a cohort study, namely that the association with a particular risk factor can be evaluated for several cancer sites simultaneously.

\section{Design and follow-up}

If it were to be a prospective study, we absolutely required it to be a large-scale study for two reasons: (a) the power should be large enough to detect moderately increased relative risks (e.g., between 1.5 and 2.0) and to study modification of the relative risks by other factors and (b) this power should be achieved in a relatively short follow-up time (i.e. five years) for the most common types of cancers (gastrointestinal, breast and lung). We considered the short follow-up period important because of the expected difficulties in raising funds for a study of very long duration and in view of the risk that hypotheses might be outdated by the end of the study. At the other hand, it was also very clear that it had to be a relatively low-cost study, since funds in the Netherlands are limited. We found the potential solution to this apparent paradox by designing an efficient study. For example, application of the case-cohort approach $(10,11)$, in which data are processed for cases and a random sample of the cohort only, would save approximately $90 \%$ of the variable processing costs, but lose only a limited amount of information. The most efficiency-enhancing features, however, were the use of existing, computerized data bases for cohort recruitment (municipal population registries) and follow-up (cancer registries). Sampling of potential cohort members from the population registries, which was permitted for scientific purposes, provided the opportunity to select the required age group (55-69 year) and receive their - correct identifying information in machine-readable form. The elaboration of the study design is described in Chapter 2.

After a grant had been awarded for a two-year pilot study (1984 and 1985), one of the major problems we faced was the follow-up by cancer registries. From the beginning of the 1980 s, the cancer registries were in the process of starting up. Although they were meant to cover the entire country in a few years' time, it was by no means certain that they would achieve their aim in time. We decided to rule out chance by (a) using PALGA, a computerized data base of pathology reports, as an additional source from which cancer cases arising in the cohort could be identified and (b) restricting cohort recruitment to those areas that were already covered by the cancer registries or PALGA near the end of 1985 . Chapter 3 deals with the method we devised to define the degree of coverage of each municipality by either a cancer registry or PALGA. Having decided to recruit the cohort in the municipalities that were sufficiently covered 
by PALGA and/or the cancer registries, the procedure how to actually link a cohort prospectively to these disease registers still needed to be worked out. Unlike the situation in, for example, Scandinavian countries where such a linkage can be conducted using a unique personal identification number, a linkage in the Netherlands bad to be based on other (non-unique) personal identifying information like family name and date of birth. In collaboration with the regional IK.L cancer registry, we developed a record linkage protocol which has consequently been adopted by the other cancer registries and PALGA. Chapter 4 describes the development of the protocol and the determination of the optimal linkage key.

\section{Assessment of exposure}

A prerequisite of a large-scale, low-cost study was, of course, an efficient method to measure exposure (dietary habits) and potential confounders. Guided by the example of the Nurses' Health Study in the USA, the most sophisticated large-scale prospective study on several types of exposure including dietary habits and a number of outcomes including cancer, we decided to use a mailed, self-administered questionnaire. A 'semiquantitative food frequency questionnaire' (12) was the only method of dietary assessment that promised to be both efficient and sufficiently valid. This type of questionnaire can be viewed as the synthesis of the concepts common among epidemiologists and those that used to prevail among nutritionists. The classic 'nutrition school' has long considered the many-day dietary record method and the comprehensive dietary history interview (13) as the only acceptable methods of individual dietary assessment, although the need for short-cut, but accurate methods was urgently felt (14). However, shorter methods were shown to be less accurate in both an absolute and a relative sense and thus often considered inadequate. Many epidemiologists, in the early studies on dietary exposure and disease, tried to assess dietary habits by asking a small number of questions about the consumption of specific foods without knowledge of the predictive value of those questions with respect to nutrient intake or other hypotheses of interest (e.g., 7). As a result, etiological epidemiological studies used either short methods of dubious or unknown validity or an elaborate assessment of dietary habits, such as the dietary history, which was consequently restricted to relatively small studies such as case-control studies (e.g., 15). The breakthrough came when epidemiologists adopted from nutritionists the notion that dietary assessment is a complicated matter that requires a methodological approach, while nutritionists started to realize that absolute accuracy of a measurement method is not a prerequisite in etiological research. A method that is sufficiently accurate in ranking study subjects with respect to the exposure of interest should be acceptable for epidemiological purposes (12), that is, when its accuracy can be estimated from a validation study. The (semiquantitative) food frequency questionnaires were (and are) developed and validated according to these acquired insights (e.g., 16-18). Chapter 5 describes how we developed and validated our food frequency questionnaire according to these principles.

In diet and cancer research, it is important that dietary assessment covers a long period preceding the clinical diagnosis of cancer (e.g., five to ten years). If dietary habits were very unstable within individuals, a single assessment would not suffice to cover such a long period. In Chapter 6 we investigated the stability of the dietary habits in our cohort over a five-year period. For this purpose, we annually repeated the dietary questionnaire in random samples from the cohort during the first five years of followup. The repeated measurements also give insight into the questionnaire's test-retest error, which, together with the results from the validation study, informs us about the 
size, type and possible consequences of the measurement error associated with the food frequency questionnaire.

An aspect that is related to the quality of the dietary assessment technique is the presence or absence of an actual exposure contrast between individuals in the study population. In particular, it has been suggested $(19,20)$ that for several dietary variables, such as fat intake, Western populations are too homogeneous to reveal associations with the risk of cancer. On the other hand, conducting a multinational prospective cohort study in both developed and developing countries simultaneously may pose severe logistic problems (e.g., exposure assessment, follow-up possibilities, standardization of methods) and consequently hamper interpretation of the results. We attempted to enlarge the exposure contrast in our cohort by studying the general population (as opposed to, for example, certain occupational groups), by recruiting the cohort from all parts of the Netherlands (as opposed to recruitment in, for example, one major city or region) and by recruitment of extra subjects with special dietary habits (vegetarians). The advantages and disadvantages of this approach are described in Chapter 2.

It has been suggested that the intake of particular nutrients is difficult to measure (e.g., selenium, of which the content in foods varies considerably, depending on the soil conditions (21-23)) and that therefore more use should be made of biological markers of dietary exposure $(20,24)$. What is often overlooked in this recommendation is that the appropriate choice and use of biomarkers in a large epidemiological study can be equally difficult. Biomarkers involving serum or urine measurements often reflect shortterm status rather than the etiologically more relevant long-term body status (12). This would necessitate multiple exposure measurements which may be difficult to accomplish in large-scale studies. Also, biomarkers that require invasive sampling techniques, such as blood sampling, may pose logistic and response problems in a healthy population. Other potential problems involve the requirement of specific transport and storage conditions. With the usual budgetary constraints this may result in relatively small cohorts being studied and consequently low power. A promising exception to this at the start of our cohort study were toenail specimens, which can be collected by participants, mailed, and stored under normal conditions $(12,25)$. In our pilot study, the mailed collection of toenail clippings turned out to be feasible in terms of sampling costs, effect on response rate, transport and storage. We found a correlation of 0.57 between selenium levels in toenails and erythrocytes, which is also a long-term marker of selenium status (26). Chapter 7 describes a study on predictors of toenail selenium levels and the association with dietary selenium intake.

A related study was carried out on the association between dietary nitrate intake and nitrate levels in urine specimens. This is an illustration of another use of biomarkers, namely for validating dietary intake measurements, if there is sufficient reason to assume that the biomarker is more valid than dietary assessment. An application with regard to nitrate is described in Chapter 8 . Because this validation study was conducted within an earlier cohort study, the relevant questions on nitrate intake could be incorporated in our baseline questionnaire.

While the assessment of dietary exposure thus forms a challenge in itself, a retrospective assessment may in addition be prone to information bias in case-control studies. Dietary habits before the diagnosis of cancer may be recalled in a biased way since cancer cases may be more aware of their diet than controls, thus influencing recall, or because dietary habits may be altered due to the disease. For the same reason, cross-sectional studies are generally considered to be inferior to longitudinal studies. However, very few data exist to confirm this presumption. In Chapter 9, we describe a study in which we compared results of a cross-sectional analysis of the association between meat consumption and cancer with a longitudinal analysis. 


\section{Statistical analysis}

A frequently used option to make efficient use of data collected in a cohort study is to analyze the study with a nested case-control approach. This involves sampling of control subjects out of the risk set whenever al case occurs. An alternative option is to use the case-cohort approach, in which a random subcohort is selected after the cahort baseline measurement, and which is used together with all emerging cases. An important advantage of this approach is that the subcohort can be used for multiple endpoints and that exposure data can be processed in advance. However, when we started the cohort study, there was no standard statistical software available for analyzing case-cohort studies. The theoretical approach to these analyses was published in 1988 (27). We developed methods for stratified and multivariate analyses of casecohort data. This is described in Chapter 10, where the methods are illustrated in the analysis of the smoking-lung cancer association in our cohort.

\section{Results}

In Chapters 11-17, results of the first analyses of the cohorit study after 3.3 years of follow-up (September 1986 - December 1989) are presented. As mentioned earlier, several hypotheses were tested to illustrate applications of the cohort data. These specific hypotheses were the following:

- The intake of total fat and various types of fat (saturated, monounsaturated and polyunsaturated fat) is positively associated with the risk of breast cancer.

- The intake of alcohol and alcoholic beverages is positively associated with the risk of colorectal cancer.

- The consumption of meat and/or fat (from meat) is positively associated with the risk of colorectal cancer.

- The association between cholecystectomy and colorectal cancer is not attributable to confounding by dietary habits or to other biases.

- Selenium status, as measured by toenail selenium levels, is inversely associated with the risk of lung, gastrointestinal and breast cancer.

\section{References}

1. Doll R, Pcto R. The causes of cancer. J Natl Cancer Inst 1981; 66: 1191-1308.

2. Mills PK, Beeson WL, Abbey DE, Fraser GE, Phillips RL. Dietary habits and past medical history as related to fatal pancreas cancer risk among adventists. Cancer 1988; 61: 2578-2585.

3. Graham S. Results of case-control studies on diet and cancer in Buffalo, New York. Cancer Res 1983; 43 (Suppl.): 2409s-2413s.

4. Willetı WC, Stampfer MJ, Colditz GA, Rosner BA, Hennekens CH, Spezer FE. Dietary fat and the risk of breast cancer. N Engl J Med 1987; 316: 22-28.

5. Miller $A B$, Howe GR, Wall $C$. The national study of breast cancer screening. Clin Invest Med 1982; 4: $227-258$.

6. Stellman SD, Garfinkel L. Smoking habits and tar levels in a new American Cancer Society Prospective Study of 1.2 million men and women. J Natl Cancer Inst 1986; 76: 1057-1063.

7. Hirayama T. A large-scale cohort study on the relationship between diet and selected cancers, of digestive organs. Gastrointestinal Cancer. Banbury Report nr. 7. Cold Spring Harbor Laboratory, 1981.

8. National Research Council. Diet, nutrition, and cancer. Washington: National Academy Press, 1982.

9. Willet WC, MacMahon B. Diet and cancer - an overview. N Engl J Med 1984; 310: 633-638 and 697-703.

10. Miettinen OS. Theoretical epidemiology. Principles of occurrence research in medicine. Netw York: Wiley; 1985. 
11. Prentice RL. A case-cohort design for epidemiologic cohort studies and disease prevention trials. Biometrika 1986; 73: 1-12.

12. Willett W. Nutritional epidemiology. New York: Oxford University Press, 1990.

13. Burke BS. The dietary history as a tool in research. J Am Diet Assoc 1947; 23: 1041-1046.

14. Marr JW. Individual dietary surveys: purposes and methods. World Rev Nutr Diet 1971;13:105-64.

15. Van ' $i$ Veer P, Kok FJ, Brants HAM, Ockhuizen T, Sturmans F, Hermus RJ. Dietary fat and the risk of breast cancer. Int J Epidemiol 1990; 19: 12-18.

16. Byers. T, Marshall $\mathbf{j}$, Fiedier $R$, Zielezny $M$, Graham $S$. Assessing nutrient intake with an abbreviated dietary interview. Am J Epidemiol 1985; 122: 41-50.

17. Block $G$, Hartman $A M$, Dresser $C$, et al. A data-based approach to diet questionnaire design and testing. Am I Epidemiol 1986; 124: 453-469.

18. Willett WC, Sampson is, Stampfer MJ, et al. Reproducibility and validity of a semiquantitative food frequency questionnaire. Am J Epidemiol 1985; 122: 51.65.

19. Higginson J. Etiologicai factors in gastrointestinal cancer in man. J Natl Cancer Inst 1966; 37: 527545.

20. Hebert JR, Miller DR. Methodologic considerations for investigating the diel-cancer link. Am J Clin Nutr 1988; 47: 1068-1077.

21. Levander OA. Considerations on the assessment of selenium status. Fed Proc 1985;44:2579-83.

22. Willett WC, Stampfer MJ. Selenium and cancer. Br Med J 1988; 297: 373-374.

23. Fan AM, Kizer K.W. Selenium. Nutritional, toxicologic and clinical aspects. West I Med 1990; 153: 160-167.

24. Hulka BS, Wilcosky TC, Griffith JD. Biological markers in epidemiology. New York: Oxford University Press, 1990.

25. Morris JS, Stampfer MJ, Willett W. Dietary selenium in humans; toenails as an indicator. Biol Trace Elem Res 1983; 5: 529-537.

26. Bausch-Goldbolm RA, van den Brandt PA, van ' 1 Veer P, van Faassen A, Hermus RJJ, Sturmans F. Diet and cancer of the breast, colon, rectum, stomach and lung: a pilot study for a prospective cohort study. Progress Report nr. 2. Zeist/Maastricht, 1985.

27. Self SG, Prentice RL. Asymptotic distribution theory and efficiency results for case-cohort studies. Ann Stat 1988; 16: 64-81. 


\title{
A LARGE-SCALE PROSPECTIVE COHORT STUDY ON DIET AND CANCER IN THE NETHERLANDS
}

\author{
Piet A. van den Brandt, ' R. Alexandra Goldbohm,2 Pueter van 't Veer,? \\ Alexander Volovics,' Rudolph J. J. Hermus' and Ferd Sturmans'
}

'Department of Epidemiology, University of Limburg. Mastricht. The Netherlands, 'Depariment of Nutrition. TNO-CIVO Toxicology and Nutntion Institute, Zeist. The Netherlands and 'Department of Medical Statistics, University of Limbure. Masstricht. The Netherlands

(Receirnd in renised form 29 hene 1989)

\begin{abstract}
In 1986, a prospective cohort study on diet and cancer was started in The Netherlands. The cohort $(n=120,852)$ of $55-69$ year old men $(48.2 \%)$ and women $(51.8 \%)$ originates from 204 computerized municipal population registries. At baseline, participants completed a self-administered questionnaire on diet and potential confounding variables. In addition, abous $67 \%$ of the parricipants provided toenail clippings. Cancer follow-up consists of record linkage to a pathology registry and to cancer registries. The initial interest is in stomach, colorectal. breast and lung tumors. A case-cohort approach is applied, in which detailed follow-up information of a random subcohort $(n=5000)$ provides an estimate of the person-time experience of the cohort. Exposure data of the subcohort will be combined with those of incident cases, yielding exposure-specific incidence rate ratios. The intraindividual variation in determinants is estimated by annually repeated measurements $(n=250)$ within the subcohort. The rationale, efficiency aspects and study characteristics are discussed.
\end{abstract}

Diet Neoplasms Epidemiologic methods Biometry Questionnaires Toenails

\section{INTRODUCTION}

The possible role of dietary factors in the etiology of human cancer continues to be a subject both of research and debate. Various estimates have been produced on the proportion of cancer cases attributable to diet and other factors [1-3]. It has been rather difficult, however, to identify specific elements of the diet as being causative or preventive. Analytical epidemiological studies on diet and cancer have been mostly of the case-control type; their results often șeem to lack consistency, which may be attributed partly to the potential for selection bias, and, particularly in dietary studies, recall bias

- Reprint requests should be addressed to: Piet A. van der Brandt. Department of Epidemiology. University of Limburg. P.O. Box 616, 6200 MD Maastricht. The Netherlands.
[4]. Considering that large-scaie randomized controlled dietary intervention trials are rarely feasible (because of financial, blinding, compliance and ethical reasons), prospective cohort studies are often proposed as the alternative method of choice. At the same time cohort studies are commonly regarded as prohibitively expensive, notably studies among the general population. The costs generally originate from recruitment of the study population, (baseline) exposure measurement and follow-up. Thus, there is a need for cost-efficient prospective cohort studies [4].

Various ongoing cohort studies on diet and cancer have been published, with widely differing characteristics. The following serves merely as a general description of the characteristics, supplemented with some examples of studies, without attempting to be complete. 
Most studies are conducted in the general population (e.g. $[5,6])$ or in a captive (occupational) subgroup of specific gender (e.g. [7]); other studies use groups with specific dietary habits (e.g. $[8-10])$. Studies may have, been started as such or have been attached to existing data collection structures as a census (e.g. [11]) or to a screening program (e.g. [12, 13]); others have made use of existing biological banks, usually without any dietary assessment (e.g. [14, 15]. Dietary assessment may include a limited (e.g. $[11,16])$ or more extensive questionnaire $[7,17]$. with the length usually inversely related to sample size. Still other studies have used a single $24 \mathrm{hr}$ dietary recall $[18,19]$ or cross-check dietary history interviews [20]. Because of their characteristics, some studies will yield data on overall dietary habits, which can hardly be translated into nutrient intakes. In addition, it might be difficult to generalize such results from studies when conducted in Japan, to Western Europe or the U.S. Studies that address the issue of nutrient intake or use biochemical markers generally take a relatively long time to yield a large number of cases because of the small study size. Indeed, a combination of dietary assessment and biological sampling on a large scale is rare [7], whereas both sources of information are likely to be complementary.

Our objective was to design an efficient, large-scale study among men and women that combines extensive dietary assessment with biological sampling, and that yields a sufficiently large number of cases within a reasonably short follow-up period. Efficiency in this respect refers to selection of the study population and area, determinant contrasts and their measurement, biological sampling. follow-up, data processing and statistical analyses. The cohort study was started in 1986 and was preceded by a pilot study in 1984 and 1985 to evaluate the feasibility of the project and develop the methods to be used. After presenting the generall outline of the study [21], the various design considerations and decisions will be discussed in detail.

\section{GENFRAL OUTLINE OF THE COHORT STUDY}

The primary purpose of the study is to investigate the effects of fats, vitamins, fiber, alcohol, selenium, nitrate, sodium and calcium on the development of gastric, colorectal, breast and lung tumors. Cancer risk associated with specific dietary patterns will also be evaluated.
These tumor sites chosen because of their suggested relationship with dietary factors (e.g. $[22,23])$ and their high incidence in The Netherlands [24].

The study is conducted among 55-69 year old men and women. Subjects originate from the general population sampled from municipal population registries. The pilot study indicated that a fairly large contrast in dietary intake exists in this population. To increase the contrast in the cohort still further, individuals with special dietary habits (e.g. vegetarians) are overrepresented. Information on determinants is obtained by a self-administered questionnaire and collection of toenail clippings. The 11-page questionnaire contains 6 pages on food habits. supplemented with questions on potential confounders and other independent risk factors. These include: smoking and occupational history, socioeconomic status, history of selected medical conditions, family history of cancer, chronic drug use, reproductive history, obesity and physical activity. The cohort is constituted by the 120,852 subjects who completed the baseline questionnaire that was sent to a total of 340,439 subjects.

Follow-up for cancer incidence will be performed by record linkage to PALGA (a data base on Dutch pathology reports) and to the cancer registries. During the first 5 years of follow-up, approximately 250 cases of stomach cancer, 450 colon, 300 rectal, 800 breast and 1200 lung cancer cases are expected to arise from this cohort, taking mortality into account $[25,26]$.

A case-cohort approach is applied, by selecting a random subcohort $(n=5000)$ from the large cohort immediately after identification of the cohort members. This subcohort is being followed up for migration and vital status by contacting the participants and the municipalities. As will be discussed, for testing the primary study hypotheses a subcohort size of 3500 is sufficiently large. Therefore, questionnaires and toenail specimens are initially processed only for a random subsample of 3500 out of the 5000 subcohort members. However, the person-time experience is also collected for the remaining 1500 subjects, whose covariate data will be processed when hypotheses regarding rare exposures responsible for a small proportion of specific tumors are of interest [27]. In the statistical analyses using the proportional hazards model [28]. stratification on year of follow-up will be employed to investigate the 


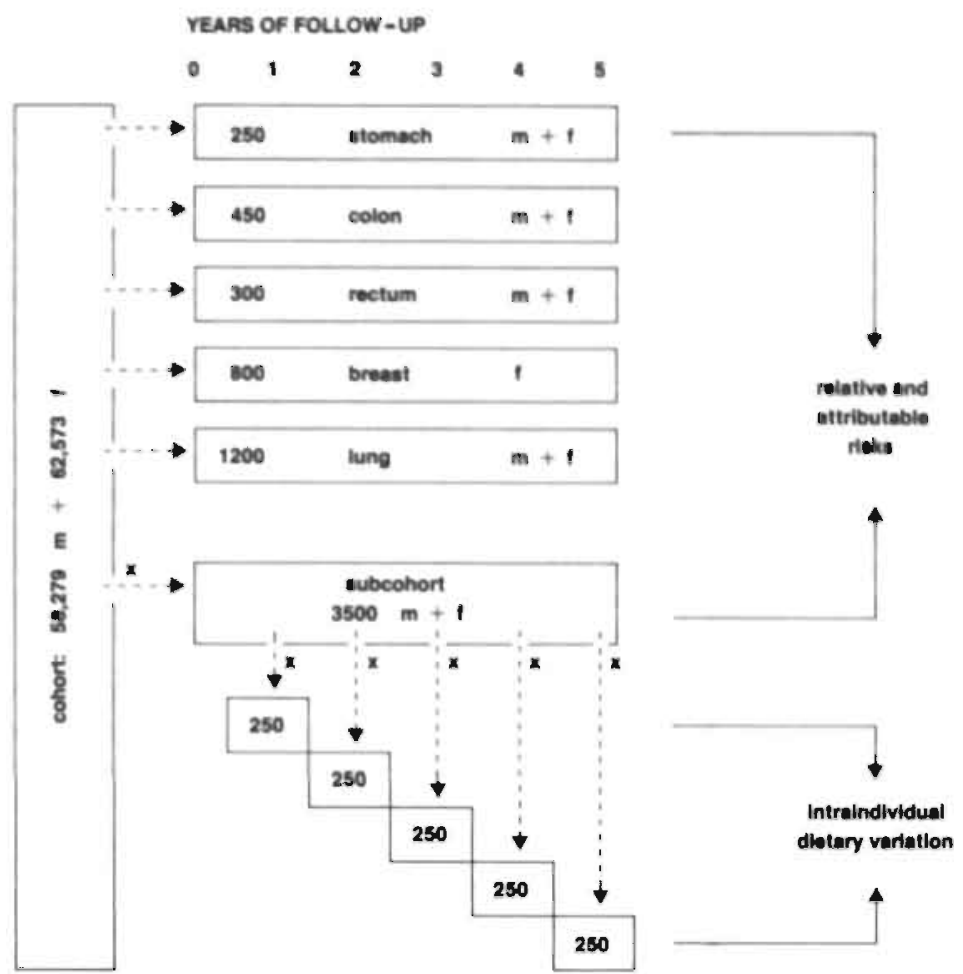

Fig. 1. Design aspects of the prospective cohort study, $x$, random sample; $m$, males; $f$, femates.

influence of possible preclinical disease at the start of the study. The intraindividual variation in determinants will be estimated by repeating the questionnaire añnually in subsamples ( $n=250$ each) of the subcohort. These design aspects are depicted in Fig. 1.

\section{SELECTION OF THE STUDY POPLLATION}

Age at entry

Because it has been suggested that diet predominantly exerts its role on the later stages rather than the early stages of tumorigenesis $[23,29]$, this cohort study is conducted among elderly people aged 55-69 years at entry. Also. younger individuals generally show less stable dietary habits, because they tend to consume more new foodstuffs [30]. In two longitudinal Dutch studies, changes in nutrient intake over a 3. year period have been. studied using interperiod correlation coefficients. The decrease in correlation among women around menopause [31] was much smaller than among adolescents [32]. Although adolescents are an extreme category in this respect, this comparison provides some evidence for higher stability at older ages.

In the age group well above 70 years, problems may occur with the dietary assessment, and there is a tendency for underreporting and less, histological verification of elderly cancer patients. Finally, a relatively short follow-up ( 5 years) of a large cohort from the selected age stratum will yield a sufficient number of cases to perform meaningful statistical analyses, i.e. minimally 300 cases per tumor site.

\section{Size and area}

In order to obtain this number of cancer cases, it was estimated that a cohort size of 150,000 subjects is required [33]. The choice of the study population is then determined largely by recruitment. efficiency and the required accuracy of identifying information in view of the proposed method of follow-up (i.e. record linkage). In The Netherlands, (computerized) municipal population registries contain highly accurate identifying information on every 
citizen, and constitute an efficient sampling frame for the general population. Since a mailed data collection procedure would be used (with a lengthy questionnaire), the aim was to start with an initial sample size of 350,000 subjects in order to establish a cohort of about 150,000 respondents.

The study area was defined in terms of municipalities satisfying the following eligibility criteria: (a) availability of a computerized population registry; (b) sufficient cancer follow-up coverage.

In 1985, 323 out of the 714 municipalities were computerized; $300(93 \%)$ of them agreed. to provide in 1986 a gender-stratified random sample of specified size, equivalent to $40 \%$ of each municipal 55-69 years age stratum. Cancer follow-up coverage was determined as follows. Recently, two sources of incident cancer cases have become available: PALGA and 9 cancer. registries. Since both PALGA and the cancer registries were not yet operating in the entire country, a list of collaborating hospitals (in
1986) was obtained. Together with data on the municipal origin of all patients admitted for cancer to these hospitals (obtained from the National Health Care Information Center), expected municipal follow-up coverage degrees were calculated per tumor site of interest. From the list of 300 computerized municipalities, 204 were selected with a coverage degree exceeding $75 \%$, yielding a tentative initial sample of almost 340,000 people. The estimated mean coverage degree for cases of any of the 5 tumor sites of initial interest was $93 \%$ in this case. Loss to follow-up due to migration out of the coverage area. (estimated at 1.9\% in 5 years) is taken into account in this estimate. The location of the 204 selected municipalities is displayed in Fig. 2. Municipal samples were selected in May-August 1986, accumulating to 339,733 subjects.

Recruitment of subjects with special dietary habits

Apart from sampling and follow-up considerations, the expected exposure contrast (and

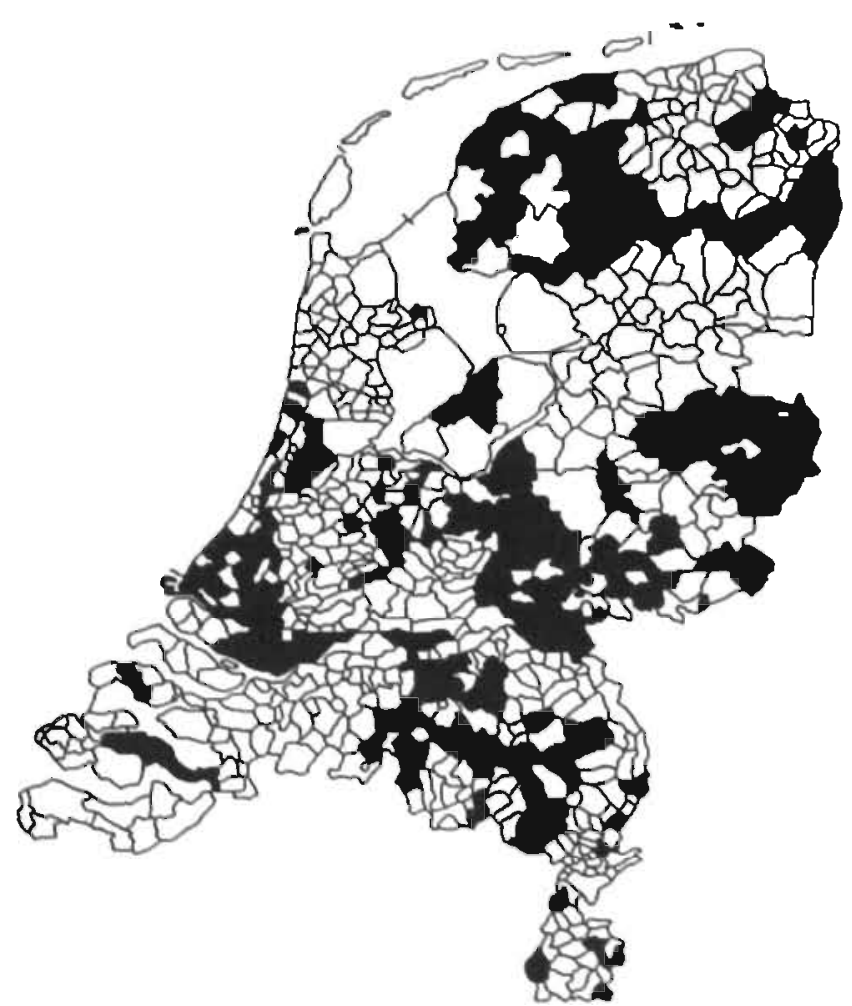

Fig. 2. The location of the participating municipalities in The Netherlands. 
its temporal stability) in the selected study population needs to be contemplated. It has been suggested that in Western populations the contrast in exposure may be small when studying individuals within one region or country [34-36]. In our pilot study we have evaluated the dietary intake in the proposed study population. which showed a rather large dietary contrast (c.g. mean $\pm \mathrm{SD}$ of dietary fat as percent of calories was $40.3 \pm 5.5 \%$ ). Within western societies, this variation can be regarded as sizeable. It can be further augmented by extending the study to other countries with different dietary habits or by overrepresentation of individuals with deviant food habits. With the former approach, problems may arise with confounders and standardization of dietary assessment, in view of different eating patterns. These problems are less serious when the second approach is used. Therefore, subjects of 55-69 years and eating meat less than twice a week were invited to participate by advertisements and leaflets in life-style magazines and health food stores located in areas covered by PALGA or the cancer registries. During the period of recruitment (April-July 1986), about 1000 persons applied; $30 \%$ of all applicants were living outside the covered areas or did not have the correct age at entry. For some of the remaining subjects, extra contacts were needed to obtain complete and correct identifying information, even though standard application forms were used. Altogether, 706 eligible "vegetarians" were recruited in this way.

\section{ASSFSSMENT OF DIETARY EXPOSURE}

\section{Choice of dietary assessment meshod}

Whereas a large inierindividual variation in exposure is desirable and variation within subjects should preferably be minimal for observational etiologic studies, it is the ratio of intrato-interindividual variation which determines whether meaningful contrasts in exposure can be studied. When this ratio is large, substantial random misclassification will result in attenuated measures of association between exposure and disease [37]. The variance ratio is in turn determined by the exposure characteristics of the study population, combined with the dietary assessment technique that, is being used. In a transitional study population with rapidly changing dietary patterns as in Japan [38] and consequently a large interindividual variation in food intake, compared to intraindividual variation, a relatively simple method may suffice [11]. Compared to the rapid and dramatic changes in Japan, changes in per capita food intake in The Netherlands are moderate [39]. Our study population will therefore show less heterogeneity (but also relatively stable dietary habits). In order to minimize misclassification, this requires an elaborate dietary assessment method, with a reference period of one year, covering seasonal variations. The dietary history can be used for this purpose, but the interview method is laborious and impractical in large-scale studies. Therefore, abbreviated methods like the (semiquantitative) food frequency questionnaire (SFFQ) have been developed, which can be self-administered. The validity and reproducibility of the SFFQ have been studied recently $[40-43]$. Combined with its feasibility, these results make it the method of choice for this cohort study. Furthermore, by repeating applications of the method annually to samples of the cohort, estimates can be made on the intraindividual variance in annual intakes. These estimates might also be used to improve estimates of the rate ratios and associated confidence intervals $[44,45]$.

\section{Construction of the dietary questionnaire}

A prerequisite for the development was that the questionnaire should be aimed at measuring the contrasts in dietary intake that exist in the cohort and it should be self-administrable. The construction of the questionnaire is described in more detail by Bausch-Goldbohm el al. [46]. Briefly, in 1984 and 1985 detailed dietary history interviews (cavering the preceding year) were conducted by trained dieticians in a gioup of 169 subjects (including 20 vegetarians) of similar age and gender structure as the cohort population. After calculation of the intakes of 15 nutrients of interest (related to the hypotheses), multiple regression analyses were employed together with residual analysis, to select those food items that predicted most of the interindividual variation in: the nutrient intakes of interest, as measured by the dietary history, Furthermore, the need for including questions on portion sizes was also evaluated by this method. Finally, the remaining list was supplemented with some items in order to maintain a. logical (dietary) structure in the questionnaire. The result was a 6-page dietary questionnaire of 175 food items, that explained the variance in nutrient intake as measured by the dietary history, ranging from $86 \%$ for vegetable fiber to 
$100 \%$ for alcohol. The validity of the final version, that was used in the cohort, is further being tested against the dietary record method and the dietary history method in ongoing substudies.

\section{Choice of biochemical markers}

Because of the potential probiems associated with the assessment of food intake, the use of biochemical markers of dietary exposures has been proposed as an objective, "hard-evidence" alternative. Although the use of biological specimens like plasma seems attractive in that the biochemical markers address the nutritional status more precisely, they nevertheless suffer from some inherent problems as well. The marker may not properly reflect long term nutritional status (c.g. [47]): large intraindividual variations in the marker content may result in a high ratio of intra-to-interindividual variation (c.g. serum cholesterol or urinary sodium). For retrospective etiologic studies. various markers may be of less value since the tumor may have altered the marker level, as has been shown for plasma Se, vitamin $\mathrm{E}$ and retinol [48-50]. In prospective sudies, the collection. storage and analyses of specimens may be prohibitively expensive, leading to smaller cohorts with decreased power and an increased risk of chance findings. A promising exception to this is toenail specimens. These reflect long term intake of several micronutrients (e.g. selenium or zinc $[51,52]$ ), and the specimens can easily be collected. transported by mail and stored at room temperature [5I]. Given these characteristics and the study size, we included the collection of toenail clippings in our study.

\section{BASEIINE EXPOSURE MEASUREMENT}

Conduct of baseline measurement and response

In September 1986, the 340,439 selected subjects were invited by mail to complete the questionnaire and collect toenail clippings. To return their completed questionnaire, respondents were offered the choice of using a business reply number (used by $33 \%$ of respondents) or (preferabiy) to provide their own stamp (used by $67 \%$ ). The acceptability of this approach had been tested in the pilot [53]. Severa! large municipalities had explicitly stated, for reasons of privacy protection, that the selected subjects could only be approached once, without use of reminders. To elevate the response rate, a nationwide publicity campaign accompanied the baseline survey. Completed questionnaires were returned by 120,852 subjects (response rate $35.5 \%$; men 34.5 , women $36.6 \%$ ). An estimated $67 \%$ of the respondents also provided toenail specimens. The first page of the questionnaire was optically scanned to define the cohort, to check specific identifying information needed for future linkage (c.g. date of birth, twinship). This page also contained questions on the presence of cancer and other conditions, overall smoking habits and special food habits (i.e. vegetarianism, veganism, etc.).

\section{Some baseline characteristics of the cohort}

The cohort is composed of 58.279 men $(48.2 \%)$ and 62.573 women $(51.8 \%)$. To examine whether the response in our study had affected the determinant distributions (e.g. did primarily non- or ex-smokers respond?), an analysis of response rates was carried out as far as the available sample data on nonrespondents permitted. Also, data from the first page of the questionnaire were used. Table I shows the response rate according to age and degree of urbanization of municipality of residence. Table 2 shows the distribution of marital status. smoking habits and overall frequency of meat consumption in the total cohort. No data on these variables are available for the nonrespondents, but for the first two variables national large-scale survey data do exist [54, 55].

Furthermore, after the cohort was identified. a random sample was selected in 1987 to validate the dietary questionnaire against the dietary record method, using 9 recording days evenly distributed over the year 1987/1988. Available data at this moment permit a comparison of the intake of several nutrients of cohort members with data from a recent national survey in which a 2-day dietary record was used [30]. Results for caloric intake and calorie providing nutrients are presented in

Tabie I. Response rate to bașelıne measurement among men and women according to age and degree of urbanization

\begin{tabular}{lcc}
\hline & \multicolumn{2}{c}{ Response rate $(\%)$} \\
\cline { 2 - 3 } Varnable & $\begin{array}{c}\text { Men, } \\
(n=58,279)\end{array}$ & $\begin{array}{c}\text { Women } \\
(n=62.573))\end{array}$ \\
\hline Age $(y r)$ & & \\
$55-69$ & 34.6 & 38.8 \\
$60-64$ & 35.1 & 36.8 \\
$65-69$ & 33.6 & 34.1 \\
Urbanization of mumcipalities & & \\
Rural & 34.5 & 39.9 \\
Semi-urbanized & 35.9 & 39.4 \\
Urbanized & 33.9 & 35.3 \\
\hline
\end{tabular}




\begin{tabular}{|c|c|c|c|c|}
\hline \multirow[b]{2}{*}{ Vanable } & \multicolumn{2}{|c|}{$\operatorname{Men}(*)$} & \multicolumn{2}{|c|}{ Women( $\%$ ) } \\
\hline & Cohort & Netherlands. & Cohort & Netherlands \\
\hline \multicolumn{5}{|l|}{ Marital status } \\
\hline Not married & 38 & $6.2^{\circ}$ & 8.4 & $77 *$ \\
\hline Divorced & 3.6 & 4.2 & 4.4 & 4.8 \\
\hline Married & 88.9 & 85.3 & 69.9 & 68.1 \\
\hline Widowed & 3.7 & 4.3 & 17.2 & 19.4 \\
\hline \multicolumn{5}{|l|}{ Smoking habits } \\
\hline Never & 9.3 & $40 t$ & 585 & 53.04 \\
\hline Ex & 488 & 510 & 20.7 & 27.0 \\
\hline Current & 41.9 & 45.0 & 208 & 20.0 \\
\hline \multicolumn{5}{|c|}{$\begin{array}{l}\text { Meat consumption } \\
\text { (freq per week) }\end{array}$} \\
\hline $0-1$ & $1.9 t$ & & $3.6 !$ & \\
\hline $2-3$ & 4.9 & & 7.7 & \\
\hline $4 \cdot 5$ & 244 & & 29.1 & \\
\hline $6-7$ & 68.8 & & 59.8 & \\
\hline
\end{tabular}

- Age category $55-69$ yr [54]

+Smoking habits in 1983, 51 + yr (55).

:No large-scale reference data available in The Netherlands

Table 3 , indicating comparable intake estimates in the two studies.

These data indicate that the response to the baseline measurement has not adversely affected determinant distributions, in the light of etiological analyses. Although of less importance, it can also be concluded that no large deviations from representativeness with respect to these variables are evident.

\section{FOLLOW-UP AND ANALYSIS ISSUES}

As mentioned earlier, follow-up for cancer in this cohori of 120,852 subjects will consist of record linkage to PALGA and the cancer registries. As an alternative to a classical cohort analysis, the covariate histories of incident cases could also be compared to those of a control group in a nested case-control study $[56,57]$. However, one would then need to wait until case occurrence for efficient matched sampling and subsequent standardized questionnaire processing for cases and control subjects. To over- come this problem, we employed a case-cohor (case base) approach, as proposed by Miettinen [58] and Prentice [59], which offers the possibility of data processing during rather than after case ascertainment. In this approach, the denominator information of the rates (i.e. the accumulated person years of the entire cohort) is estimated using a subcohort of sufficient size, while cases are enumerated for the entire cohort (numerator information).

\section{Required size of subcohort}

Determination of the required subcohort size (3500) for testing the primary hypotheses in the case-cohort study was initially based on asymp. totic relative efficiency comparisons for risk ratios. Efficiency results regarding rate ratios of Self and Prentice [60] had not yet been published at the time the decision on size had to be made. The asymptotic variances for the logarithm of the risk ratios estimated from the classical full cohort design (denoted by VCO) and from the case-cohort design (VCC) were calculated

Table 3. Mean caloric intake and its contributors among men and women in the cohort. as calculated from 9-day dietary records, and in The Netherlands (2-day dietary record)

\begin{tabular}{lccccc}
\hline & \multicolumn{2}{c}{ Men } & & \multicolumn{2}{c}{ Women } \\
\cline { 2 - 3 } \cline { 5 - 6 } Variable & $\begin{array}{c}\text { Cohort } \\
(n=60)\end{array}$ & $\begin{array}{c}\text { Netherlands" } \\
(n=431)\end{array}$ & & $\begin{array}{c}\text { Cohort } \\
(n=52)\end{array}$ & $\begin{array}{c}\text { Netherlands* } \\
(n=460)\end{array}$ \\
\hline Caloric intake (kcal) & 2408 & 2564 & & 1981 & 1946 \\
Fat $(\%$ energy) & 40.0 & 41.2 & & 40.7 & 41.1 \\
Protein (\% energy) & 14.1 & 13.7 & & 14.4 & 14.9 \\
Carbohydrates (\% energy) & 40.9 & 40.2 & & 42.1 & 41.2 \\
Alcohol (\% energy) & 5.0 & 5.0 & & 2.8 & 2.7 \\
\hline
\end{tabular}

- Ministry of Welfare, Public Health and Culture [30] 
under simplifying assumptions: no competing risks, negligible loss to follow-up, and for a single dichotomous exposure variable. With $S$ being the ratio of the subcohort size to the expected number of cases, VCO and VCC were calculated for a range of values of relative risk (RR; $0.1-10)$; control exposure probability ( $\alpha$; 2-90\%), expected 5-year cumulative incidence (CI; $0.2-2 \%)$ and $S(1-25)$. As an example of a typical situation for a dietary exposure, Fig. 3 shows a plot of VCC against values of $S$ for $\mathrm{RR}=2, \alpha=33 \%, \mathrm{Cl}=0.2 \%$ (female rectum cancer), $\mathrm{CI}=0.4 \%$ (male stomach $)$ and $\mathrm{CI}=2 \%$ (male lung), respectively. This figure illustrates that the variance estimate (or confidence interval) for the less common cancers will never be as small as that for lung cancer. The graph further indicates that for female rectum cancer the decrease in variance is minimal when $S$ is increased over 16, while for male stomach and lung cancer this value of $S$ is approximately 8 and 2, respectively.

For the various tumor sites, the relative efficiency VCO/VCC $[27,61]$ was then considered. Figure 4 shows VCO/VCC as a function of RR for $\alpha=33 \%, C I=0.4 \%$ and $S=1,2,4,8,16$ and 25 . Figure 4 indicates that $\boldsymbol{S}$-values of 8 or higher are clearly sufficient over the entire range of RR-values. Similar results were obtained for the other tumor sites.

After considering the relative efficiencies under various conditions for various subcohort sizes and the added cost of processing additiona! ỵuestionnaires, we decided to choose a random subcohort of 3500 subjects. For most tumor sites, $S$-values or more are attained with this subcohort (e.g. 9 for male stomach and 16 for

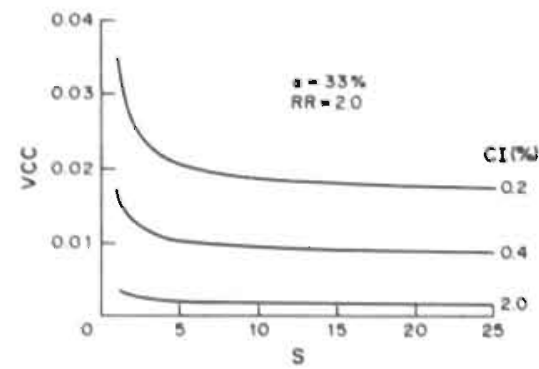

Fig. 3. The variance of $\log R R$ as a function of the ratic (subcohort:cases) for 3 cumulative incidence rates, using the case cohort method. VCC, variance of logRR with the case cohort method: $S$, ratio of subcohort:cases, $\mathrm{CI}$. cumulative incidence; $x$, control exposure probability, RR. relative nsk.

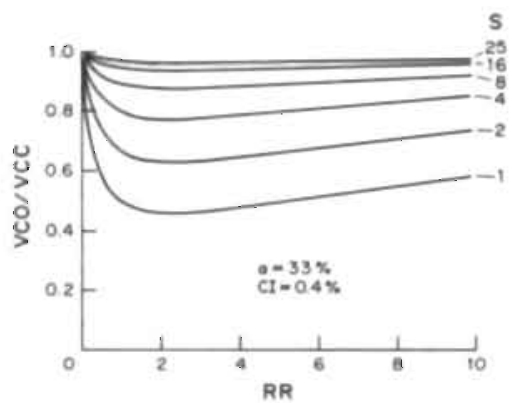

Fig. 4. The relative efficiency of the case-cohort vs full cohort analysis as a function of RR, for various subcohort sizes. VCO, varuance of $\log R R$ with the full cohort method. VCC, variance of $\log R R$ with the case-cohort method; $S$. ratio of subcohort:cases; $\mathrm{Cl}$, cumulative incidence; $a$. control exposure probability; RR, relative risk.

female rectum cancer): for male lung and female breast cancer the value of $S$ would be 1.5 and 2 , respectively. While the associated efficiencies for the latter tumor sites would be $50-60 \%$ for various combinations of $\alpha$ and $\mathbf{R} \mathbf{R}$, it should be kept in mind that the confidence intervals would still be much smaller than for less frequent cancer sites (see Fig. 3).

To check the efficiency of the chosen subcohort size with regard to rate ratios and more realistic assumptions, parametric relative rate regression models for case-cohort. studies were formulated. Based on these models, simulation studies were performed; the results were in accordance with those concerning the risk ratio.

\section{DISCESSION}

We have started a prospective study on diet and cancer in a general population cohort of 120,852 men and women, in which determinant information from questionnaires and from toenail clippings is analyzed together with cancer incidence, using the case-cohort method. Conducting a study among the general population has the disadvantage of possible incomplete control for confounding of e.g. occupation as opposed to cohorts that are restricted in this sense (e.g. [7]). On the other hand, when these confounders are measured accurately, it also provides an opportunity to evaluate their effect modification (e.g. of occupation). The choice is usually determined, however, by the availability of specific population rosters and the possibilities for follow-up. The presence of both municipal population registries and 
cancer registries in The Netherlands offered the opportunity for efficient recruitment and follow-up of the present cohort.

Another aspect that contributed to the efficiency was the increase in determinant contrast in the cohort by the intentional overrepresentation of vegetarians, albeit to a small extent. The somewhat disappointing experience in the recruitment of these individuals illustrates the inefficiency of obtaining large samples with accurate data through advertisements, as opposed to sampling from computerized population rosters with high quality data, needed when cancer follow-up is based on record linkage.

Loss to follow-up is the primary source of potential selection bias in prospective cohort studies (provided, it is differential across determinant strata $[62,63])$. Therefore it should be minimized, like in experimental studies. Hence, the study area and population in the present cohort study were chosen in a way to ensure sufficient follow-up coverage. Recruitment of a large general population cohort in the way described, implies an incomplete response to the baseline measurement. Bias in determinant distributions due to nonresponse has no serious implications for ratio estimates, even though respondents generally show lower mortality or disease experience during follow-up than nonrespondents (e.g. [64, 65]). In studies that have addressed the issue of nonresponse, odds ratio estimates were not significantly different between participants and non-participants, although both groups exhibited (largely independent) differences in determinant distributions and disease experience $[65,66]$. In fact, the distribution of risk factors may even become more favorable for etiologic studies due to response at baseline. This potentially increased efficiency is also why intentional overrepresentation of vegetarians, was pursued in the present study, and why in an experimental situation subjects are allocated equally to determinant strata. Data on demographic variables, smoking and dietary habits were presented indicating that the response did not adversely affect determinant distributions in the present cohort. To evaluate whether differential loss to followup occurs, we will compare the determinant profile of those lost to follow-up with other participants.

An elderly cohort was selected because dietary habits (and their contrasts) are stabilized, and such a cohort will yieid sufficient cases for meaningful analyses within a reasonable time period. It can be argued that evaluation of nutritional determinants of cancer acting early in life $[67]$ cannot be evaluated. with this approach. Since it has been suggested that various dietary factors act in later stages of carcinogenesis and a large-scale study among a cohort of e.g. adolescents would be timeconsuming with the need to consider intermediate (dietary) events also, this potential drawback was accepted. Together with other ongoing studies and studies that will investigate the role of diet in the earlier stages of carcinogenesis, this study will contribute to a better understanding of the type, timing and weight of the influence diet may have on human cancer development

Acknowiedgements - This work was supported by grants from The Netheriands Cancer Foundation and the Ministry of Welfare. Public Health and Cultural Affair. The author want to thank M. Bethlehem, H Brants, C. van Deursen. G. Franssen, H. Hofhuis, A. Huldij. M de Leeuw, H. van Montfort, A. Schiphorst, R. Schmeity, H Smit and $G$. Snijders for their assistance in the conduct of this study, and F. J. Kok for useful comments on this manuscript.

\section{REFERENTES}

1. Wynder EL, Gori GB. Contribution of the environ ment to cancer incidence: an epidemiologic exercise J Nat Cancer Inst 1977, $58: 825-832$

2 Higginson I. Muir CS. Environmental carcinogenesis misconceptions and limitations to cancer control J Natl Caneer Inst 1979;63:1291 1298

3. Doll R. Peto R. The causes of cancer I Nall Cancer tast 1981: 66: 1191-1308

4. Zaridze. DG. Muir CS. McMichael AJ Diet and cancer. value of different types of epidemiological studies. Nutr Cancer 1985; 7: 155-166.

5. Graham S. Results of case-control studies on diet and cancer in Buffalo, New York. Cancer Res 1983; 43 (Suppl.): $2409 \mathrm{~s}-2413 \mathrm{~s}$.

6. Kvale G. Bjelke E, Gart JJ Dietary habits and lung cancer risk. Int I Cancer 1983; 31: 397-405

7. Willett WC, Stampfer MJ. Colditz GA, Rosner BA. Hennekens CH. Speizer FE. Dietary fat and the risk of breast cancer. N Engl J Med 1987: 316: 22-28

8. Phillips, RL Snowdoni DA Association of meat and coffee use with cancers of the large bowel, breasr and prostate among Seven-Day Adventists preliminary results. Cancer Res 1983; 43 (Suppl.): 2403s-2408s

9. Enstrom JE. Cancer mortality among Mormons in California during 1968-75. J Natl Cancer Inst 1980; 65: 1073-1082

10. Kolonel LN, Nomura AMY, Hinds MW, Hirohata T, Hankin JH. Lee J. Role of diet in cancer incidence in Hawaii Cancer Res 1983, 43(Suppl.): 2397s-2402s.

11. Hirayama T. Diet and cancer. Nutr Cancer 1979; 1 : 67-81

12. Miller' AB. Howe GR, Wall C. The national study of breast cancer screening. Clin Invest Med 1982; 4 : 227-258. 
13. Van Noord PAH, Collette HJA, Maas MJ, De Waard F. Selenium levels in nails of premenopausal breast cancer patients assessed prediagnostically in a cohortnested case-referent study among women screened in the DOM project. Int J Epidemiol 1987; 16 (Suppl.) 318-322

14. Salonen JT, Salonen R, Lappetefainen R, Maenpaa PH. Alfthan G. Puska P. Risk of cancer in relation to serum concentrations of selenium and vitamins $A$ and E. matched case-control analysis of prospective data. Bir Med J 1985; 290: 417-420.

15. Kok FJ, De Bruin AM, Hofman A, Vermeeren R: Valkenburs HA. Is serum selenium a risk factor for cancer in men only? Am J Epldemiol 1987, 125: 12-16.

16. Stellman SD, Garfinkel L. Artificial sweetener use and one-year weight change among women. Prer Med 1986; 15: 195-202

17. Paganini-Hill A, Chao A, Ross RK, Henderson BE Vitamin $A$, beta-carotene, and the risk of cancer: a prospective study, I Nat Cancer Insil 1987; 79 443-448.

18. Jones DY, Schatzkin A, Green SB, Block G, Brinton 1.A. Ziegler RG. Hoovet. R. Taylor PR. Dietary fat and breast cancer in the National Health and Nutrition Examination Survey - Epidemiologic Follow-up Study J Natl Cancer Insf 1987; 79, 465-471

19 Nomura A. Heilbrun LK. Stemmermann GN Prospective study of coffee consumption and the risk of cancer. I Natl Cancer Inst 1986; 76; $587-590$

20. Kromhout D, Bosschieter EB, De Lexenne Coulander C. Dietary fibre and 10-year mortality from coronary heart discase, cancer, and isll causes; the Zutphen Study. Lancet 1982; u; 518-521.

21. Van den Brandt PA, Goldbohm RA, van 't Veer P. Hermus RJJ. Sturmans F. Dietary habits and the aetiology of cancer. Int J Epidemiol 1988; 17: 472.

22. Palmer S. Dier, nutrition and cancer. Prog Food Putr Sei 1985; 9: 283-341.

23. Willet! WC, MacMahon B Die! and cancer-an overview (1wo parts). N Ent J Med 1984; 310;633-639 and $697-703$.

24. Muir C, Waterhouse J, Mack T, Powell J, Whelan S, Eds Coneer lincidence in Fire Continents, Vol. V. Lyan. JARC Soi. Publ.: 1987: 88

25. Central Blureau of Statistics. Kankermorbiditeit en

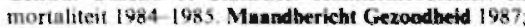
(i.) $5-21$

26. Central Bureau of Statistics. Sterfte, 1982-1986. Masalsentistied Bevolking 1987; 9: 36-39

27. Bresiow NE, Day NE. Siathical Mertods in Cance Research. 11. Th Destro and Anulysis, of Cobor Studies. Lyon: IARC Si. Publ.; 1987: 82.

28. Cox DR Regression models and life tables fwith discussion). J Et Stat Soc B 1972; 34: 187-220.

29. National Academy of Sciences, National Research Council, Committee on Diet, Nutrition, and Cancer: Diet, Netrition, end Cencer, Washington: National Academy Press, 1982

30. Ministry of Welfare, Public Health and Culture, Ministry of Agriculture and Fisheries. Wat aet Nederlagd? Rijswijk. The Netherlands, 1988.

3. Van Beresteyn ECH, Van't Hof MA, De Waard H. Dekker PR, Neeter R, Winkeldermaat Hi, Visser RM. Schaafsma G. Van Schiak M. Duursma SA. Design and data quality of mixed longitudinal study to clucidate the role of dietary calcium and phosphorus in bone mineralization in pre-, peri- and post-menopausal women. An J Clin Nutr 1986; 4: 538-548.

32. Post GB Nutrition in adolescence. Dissertation. Wageningen, 1989

33. Central Bureau of Statistics. Kanker 1978-1979; morboditeit en mortaliteit. Maandstatistiek Bevolking Volksgerondheid 1981; 29: 46-61.
34. Hacnszel W, Kurihara M, Locke FB, Shimuru K. Segi M. Stomach cancer in Japan. J Natl Cancer Inst 1976; 56: 265-274

35. Higginson J. Etiological factors in gastrointestinal cancer in man. I Natll Cancer Inst 1966; 37: 527-545.

36. Hebert JR, Miller DR. Methodologic considerations for investigating the diet-cancer link. Am J Clin Nutr 1988; 47: 1068-1077

37. Liu K, Stamler J, Dyer A. MeKeever P, McKeever J. Statistical methods to assess and minimize the role of intraindividual variability in obscuring the relationship between dietary lipids and serum cholesterol. J Chron Dis 1978; 31 : $399-418$.

38. Oiso $T$. Incidence of stomach cancer and its relation to dietary habits and nutrition in Japan between. 1900 and 1975. Cancer Res 1975: 35: 3254-3258

39. Netherlands Nutrition Council. Richtijnen goede vooding. Voeding 1986; 47: 159-181'

40. Rohan TE, Potter JD Retrospective assessment of dietary intake: Am A Epidemiol 1984; 120: 876-887

41. Willett WC, Sampson L. Stampfer MJ, Rosner B, Bain C. Witschi J, Hennekens CH. Speizer FE. Reproducibility and validity of a semiquantitative food frequency questionnaire. Am J Epidemiol 1985; 122: 51-65.

42. Willett WC, Reynolds RD, Cottrell-Hochner. S. Sampson L. Browne ML. Validation of a semi-quantitative food frequency questionnaire: comparison with a i-year dier record. JADA 1987; 87: 43-47.

43. Jain MG, Harrison L. Howe GR, Miller AB. Evalua. tion of a self-administered dietary questionnaire for use in a cohort study. Am J Clin Nutr 1982; 36 931-935

44. Clayton DG. using test-retest reliability data to improve relative risk: estimates: an application of latent class analysis. Stat Med 1985; 4: 445-455.

45. Marshafl JR, Graham S. Use of dual responses to increase validity of case-control studies. J Chron Dis 1984: 37: 125-136.

46. Brusch-Goldbohm RA, van den Brandt PA, van 't Veer P. Sturmans F. Hermus RJI. Results of the methodological study for the design of a simplified. self-administered. questionnaire, In Riboli $E_{1}$. Sarace R., Eds. Dikt, Hormones and Cancer: Mithodological Issues, for Prospective Studies. Lyon: IARC Technical Report: 1988: 4: 79.89

47. Hodges RE, Hood J. Canham JE, Sauberlich HE Baker. EM. Clinical manifestations of ascorbic acid deficiency in man. Am $J$ Clin Nutr 19.71: 24: 432-443.

48. Robinson MF, Godfrey PJ, Thomson CD, Rea HM Van Ri AM. Blood selenium and glusathione peroxidase activity in normal subjects and in surgical patients with and without cancer in New Zealand. An .I Clin Nutr 1979; 32: 1477-1485.

49. Wald N, Boreham i, Bailey A. Serum retinof and subsequent risk of cancer. B $\boldsymbol{J}$ J Cancer 1986; 54 957-961.

50. Wald NJ. Thompson SG, Densem JW, Boreham J. Bailey A. Serum vitamin $E$ and subsequent risk of cancer. Br J Coeser 1987: 56: 69-72

51. Morris JS, Stampfer MJ, Willett W. Dietary selenium in humans. Toenails as an, indicator. Bioll Trace Elem Res 1983; 5: 529-537

52. Schrauzer GN. White DA. Schneider Cl. Cancer mortality correlation studies IV: Associations with dietary intakes and bloodlevels of certaint trace elements, notably Se-antagonists. Bioinor Chem 1977; 7 ; 35- 54

53. Van den Brandt PA. Bausch-Goldbohm RA, van. 't Veer P. Hermus RJJ, Sturmans F. The Dutch prospective cohort study on diet and cancer In: Riboli $E$ Saracci $R$, Eds. Diet, Hormones and Cancer: Methodological Issues for Prospective Studies. Lyon: IARC Technical Report; 1988: 4: 18-27. 
54. Central Bureau of Statistics. Statistisch Zakboek 1986 The Hague: Staatsuitgeveri, 1986.

55. Van Reek J, MJ Drop. H Adriaanse. Stoppen met roken sinds 1958. Tydschriff Alcohol Drugs 1985: 11. $168-173$

56. Mantel N. Synthetic retrospective studies and related topics. Blometrics 1973; 29, 479-486

57. Thomas DC. Addendum to a paper by Liddell FDK. McDonald JC \& Thomas DC. J R Stat Soc A 1977. 140: $483-485$

58. Miettinen OS. Theoretical Epidemiology. Principles of Occurrence Research in Medicine. New York: Wiley, 1985.

59. Prentice RL. A case-cohort design for epidemiologic cohort studies and disease prevention trials. Biometrika 1986; 73: 1-12.

60. Self SG. Prentice RL. Asymptotic distribution theory and efficiency results for case-cohort studies. Ann Ștat 1988: $16: 64-81$

61. Whittemore AS, McMillan A. Analysing occupational cohort data: application to US uranium miners.
In: Prentice RL, Whittemore AS, Eds. Environmeatal Epidemiology: Rish Arsewement. Philadelphia: SIAM: 1982: 65-81.

62. Greenland S. Response and follow-up bias in cohort studies. Am J Epidemiel 1977; 106: 184-187.

63. Kleinbaum DG, Kupper L. Morgenstern H. Epidemiologic Research: Principles and Quantitative Methods, Belmont, California: Lifetime Learning: 1982.

64. Doll R. Hill AB. Mortality in relation to smoking: ten years' observations of British doctors. Br Med $\mathbf{I}$ 1964. 1: $1399-1410$

65. Heilbrun LK, Nomura A, Stemmermann GN. The effects of nonresponse in a prospective study of cancer. A.m. J Ypidemiel 1982; 116:353-363.

66. Austin MA, Criqui MH, Barrett-Connor E, Holdbrook MJ. The effect of response bias on the odds ratio. Am fi Epldemiol 1981; 114: 137-143.

67. Ross MH. Lustbader ED. Bras G. Dietary practices of early life and spontaneous tumon of the rat. Nutr Cancer 1982; 3: 150-167. 



\title{
Chapter 3
}

\section{Estimation of the coverage of municipalities by cancer registries and PALGA using hospital discharge data*}

\author{
R. Alexandra Goldbohm ${ }^{1,2}$, Piet A. van den Brandt', Elisabeth Dorant ${ }^{2}$.
}

1) TNO Toxicology and Nutrition Institute, Zeiss, the Netherlands; 2) University of Limburg, Department of Epidemiology, Maastricht, the Netherlands

\begin{abstract}
In a large-scale prospective cohort study on diet and cancer, which has been Inltiated in September 1986, follow-up for cancer is being conducted through record linkage with the regional cancer reglsirtes (CR) and with PALGA, a data base of pathology reports. During the first few years of the study, however, neither the CR nor PALGA operated nation wide. Since the cohort was to be recruited from samples drawn from a large number of municipal population registries, selection of municipalities according to the degree in which their inhabltants were "covered" by CR and PALGA would minimize loss to follow-up.

Hospital discharge data, which include diagnosis, age, sex and residence of each discharged (or deceased) patient, were used to estlmate for each municlpallty the proportion of hospitalized patients admitted to those hospitals that were expected to participate in either the CR or PALGA at the date the cohort study was planned to start A minimum coverage of $75 \%$ was used as criterion for selection of a munlcipality. Of the 204 municipalities selected, 188 attained more than $90 \%$ coverage; the mean coverage of the sampled cohort at the start of the study was estimated at $94.3 \%$. The analysis was repeated several years after the start of the cohort study to assess retrospectively the actual coverage of the cohort over time. The mean coverage appeared to increase from $98.5 \%$ at the start of the study to $100 \%$ in 1988 .
\end{abstract}




\section{Introduction}

In 1986, a large-scale prospective cohort study was initiated in the Netherlands, which investigates the association between dietary habits and the risk for (stomach, colorectal, lung and breast) cancer among more than 120,000 men and women aged 55-69. After the baseline administration of a mailed questionnaire in September 1986, follow-up for cancer was to be accomplished by the nine regional, population-based cancer registries (IKN, IKO, IKMN, IKA, IKST, IKW, IKR, IKZ and IKL) and PALGA (Pathologisch Anatomisch Landelijk Geautomatiseerd Archief), a Dutch data base of pathology reports (1).

The majority of cancer registries functioning in the Netherlands started to register in a limited number of hospitals in the period between 1982 and 1986 and gradually extended their registration activities to all hospitals in their own region by 1989 (2). One registry (IKZ-SOOZ) dates from 1953. In January 1985, the PALGA data base included 28 of 70 pathology laboratories in the Netherlands, accounting for approximately $50 \%$ of all pathology reports (3). In June 1990, all laboratories had joined PALGA.

Considering the incomplete coverage of the Netherlands in 1986 as described above, it was essential for sufficient follow-up to recruit the cohort from geographic areas in which either one of the cancer registries (CR) or PALGA was operational from the start of the study. Since it was decided for practical reasons to sample the cohort from the municipal population registries, the question arose how to determine the degree of coverage of each Dutch municipality by the CR and PALGA. The availability of a nation wide data base of hospital discharge data enabled us to answer that question. This paper describes the methods to estimate the coverage of the municipalities, the subsequent selection of municipalities for cohort recruitment, and the actual coverage of the cohort sample as assessed retrospectively from the hospital discharge data.

\section{Methods}

In 1985, during the planning stage of the cohort study, a list of hospitals was composed that were either participating in one of the registries already or very likely would do so in 1986. The information concerning participation of hospitals was obtained from each of the cancer registries. Since most of the pathology laboratories are connected to and working for one or more hospitals, a list of hospitals linked with PALGA was also drawn up.

The Dutch Center for Health Care Information (SIG) maintains the National Medical Registry (LMR), a data base of hospital discharge data (3). The data base contains (anonymous) data on each patient discharged from or deceased in the hospital. The following data were relevant to our study: hospital code, diagnosis (ICD-9 code), sex, age and municipality of residence, and, in case of malignancy, whether it concerned a first or a repeated admission for that specific diagnosis. For privacy reasons, we could not obtain the original data. Therefore, we provided the SIG with the two lists of registering hospitals and received in return the data, aggregated by municipality of residence and restricted to the age group 55 to 69 and to diagnoses of digestive tract, lung and breast cancer (ICD-9 codes 151, 153, 154; 162; and 174-175 respectively). For each municipality, diagnosis and sex, the following data were provided: total number of discharges in (a) CR hospitals, (b) PALGA hospitals, (c) CR and/or PALGA hospitals and (d) all hospitals in the country. Similar data for first admission for the disease were also available. 
From these data we calculated for each municipality the diagnosis- and sex-specific proportion of patients discharged from "registering" hospitals, i.e. a, b, or c divided by d. These proportions can be considered as the coverage of a municipality by the respective registries. The proportion pertaining to coverage by either a CR or PALGA (c/d) was used as a selection criterion for cohort recruitment. Besides degree of coverage, also other criteria for eligibility of a municipality for cohort recruitment were applied. These criteria were: (a) availability of a computerized population registry, administered by one of nine regional computer centers and (b) permission to draw a $40 \%$ sample from the men and women aged 55 to 69 in their population registry. A higher sampling fraction was thought to jeopardize the participation of municipalities. Of the 323 (from a total of 714 ) municipalities that met criterion a, $23(7 \%)$ refused participation, leaving 300 municipalities eligible for selection according to coverage. Based on the number of cancer cases to be expected in the cohort study after five years of follow-up, the required sample size was set at 350,000 . The aim of the analysis was to select municipalities in descending order of coverage until the required sample size was achieved, taking into account that the average coverage of the sample should not be lower than $90 \%$. The calculations were performed for all data as well as for those pertaining to first admission only.

The selection of municipalities for the cohort study was based on the LMR data base of 1983, the most recent one available in the planning stage of the cohort study. After the start of the study, the analysis was repeated using the combined LMR data base of 1987/1988 and hospital lists retrospectively updated according to their actual registration status on January 1, 1987 and January 1, 1988. The situation at the start of the study (October 1, 1986) differed from that in 1987 for three hospitals. Coverage of the municipalities involved was corrected according to these hospitals' contribution to the total number of hospital beds in the region.

\section{Results}

Table 1 displays the number of municipalities and the achievable sample size according to different cut-off points of anticipated coverage. Complete coverage appeared to be attained for 219 municipalities, corresponding to a sample size of 256,000 . A sample size of 350,000 could be achieved at a cut-off point between 70 and $80 \%$. The ultimately chosen cut-off point of $75 \%$ corresponded to a sample size of 342,000 and comprised 249 municipalities. As a result of consolidation of small municipalities into larger ones during the period between planning and sampling, the actual sample was drawn from 204 municipalities. The mean anticipated coverage of the sample amounted to $94.3 \%$. When it was taken into account that part of the participants in the cohort study would move to municipalities that were not yet sufficiently covered, the anticipated coverage decreased to $93.0 \%$.

The coverage of the municipalities participating in the cohort study as determined from the actual registration situation on January 1, 1987 (three months after the start of the cohort study) is presented in Figures 1 and 2. Figure 1 shows the coverage by the $\mathrm{CR}$ and PALGA combined, while Figure 2 displays the coverage by the CR alone. The actual coverage of the cohort appeared to be $99.5 \%$, much higher than the value of 94.3\% anticipated before the start of the study. The coverage of three municipalities, however, did not attain the initial cut-off point of $75 \%$. Neither the CR nor PALGA attained sufficient coverage alone ( $88.6 \%$ and $82.5 \%$, respectively). 
Table 1. Number of municipalities and sample size according to cut-off point of anticipated coverage by the cancer registries and PALGA.

Cut-off point (\%)

Number of municipalities*

Sample size†

$\begin{array}{ccc}\text { No cut-off } & 300 & 491000 \\ 70 & 251 & 376000 \\ 80 & 244 & 339000 \\ 90 & 237 & 328000 \\ 100 & 219 & 256000\end{array}$

- 300 of the 714 municipalities were eligible for the analysis.

$\uparrow$ Assuming a sampling fraction of $40 \%$ (age group 55-69), except for one large municipality, which only permitted a $20 \%$ sample.

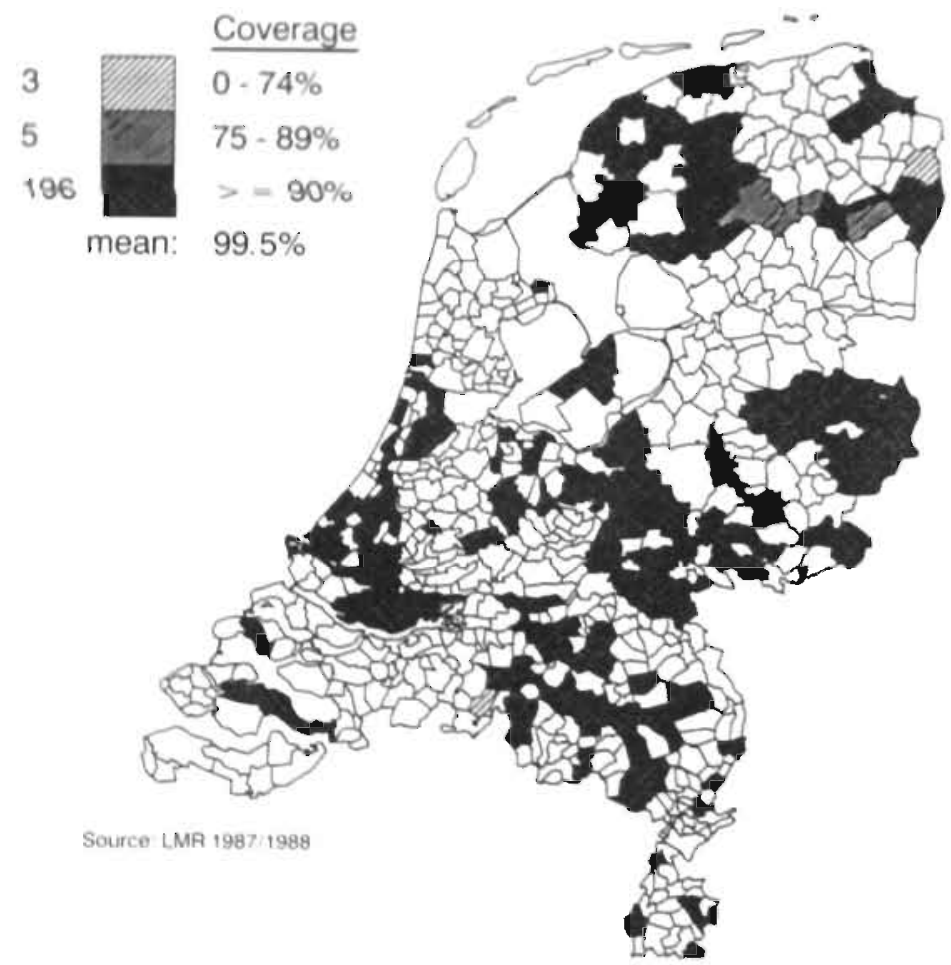

Figure 1. Actual coverage of the cohort municipalities by cancer registries and PALGA, January 1, 1987. (Blank municipalities are not participating in the study.) 


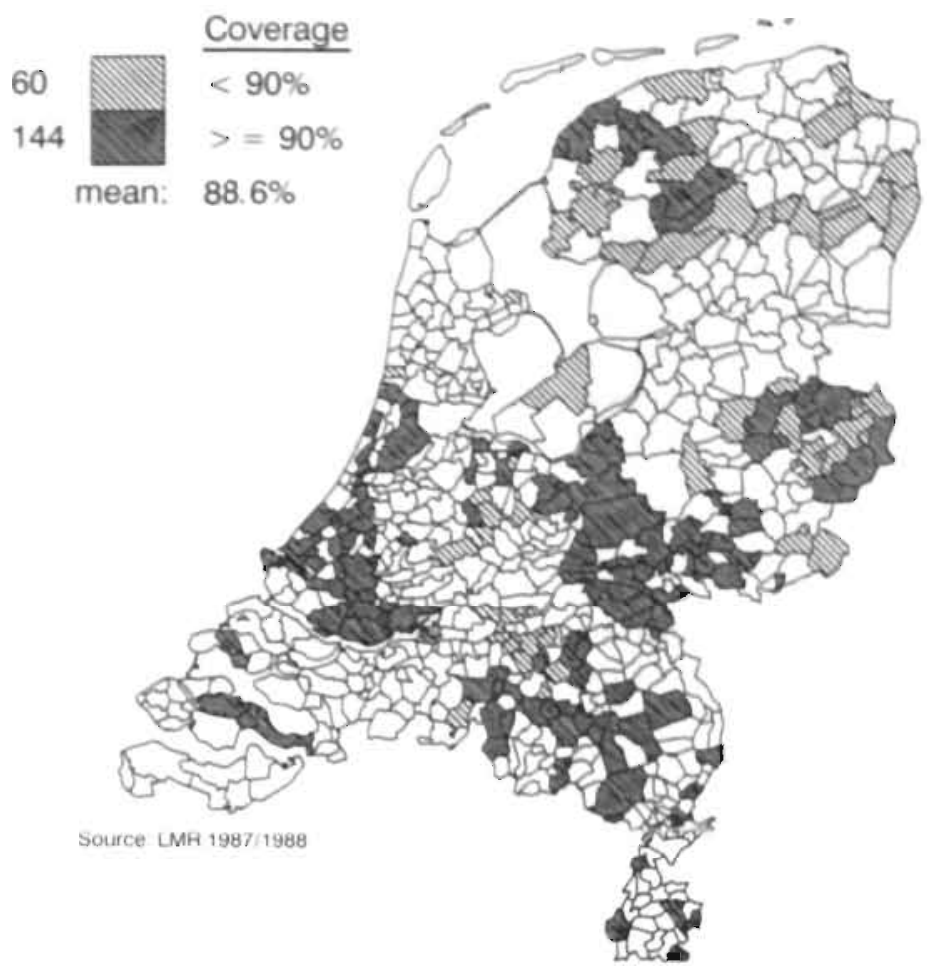

Figure 2. Actual coverage of the cohort municipalities by the cancer registries, January 1,1987. (Blank municipalities are not participating in the study.)

Tablle 2 gives the site- and sex-specific coverage on January 1, 1987. No large differences were detected between any of the cancer sites nor between men and women. Furthermore, the mean coverage was similar whether all admission data or those pertaining to first admissions were used.

Table 2. Actual coverage (\%) by cancer registries and PALGA on January 1, 1987 of the cohort sampling population specified for cancer site and sex, calculated for all admissions and first admissions only.

\section{Men}

Site

ICD code

All admissions

First admissions

99.5

99.5

99.2

99.2

99.1

Lung

154

Breast

$174+175$

All sites (both sexes)
995

99.4

Women

All admissions First admissions.

$\begin{array}{rr}99.3 & 99.3 \\ 99.7 & 99.7 \\ 100.0 & 100.0 \\ 99.3 & 99.2 \\ 99.4 & 99.5\end{array}$


Table 3 shows, however, minor differences within municipalities between coverage degrees calculated from all admissions and those calculated from first admissions only.

Table 3. Classification of cohort municipalities ( $n=204$ ) according, to coverage on January 1, 1987: all admissions versus first admissions only.

\section{All admissions}

First admissions

$0-74 \%$

$75-89 \%$

$\geq 90 \%$

$0.74 \%$

$75-89 \%$

$\geq 90 \%$
3

0

0
0.

3

2
0

4.

192

Figure 3 summarizes the anticipated coverage of the cohort and the development of the actual coverage from the start of the study up to January 1, 1988, when complete coverage was reached.

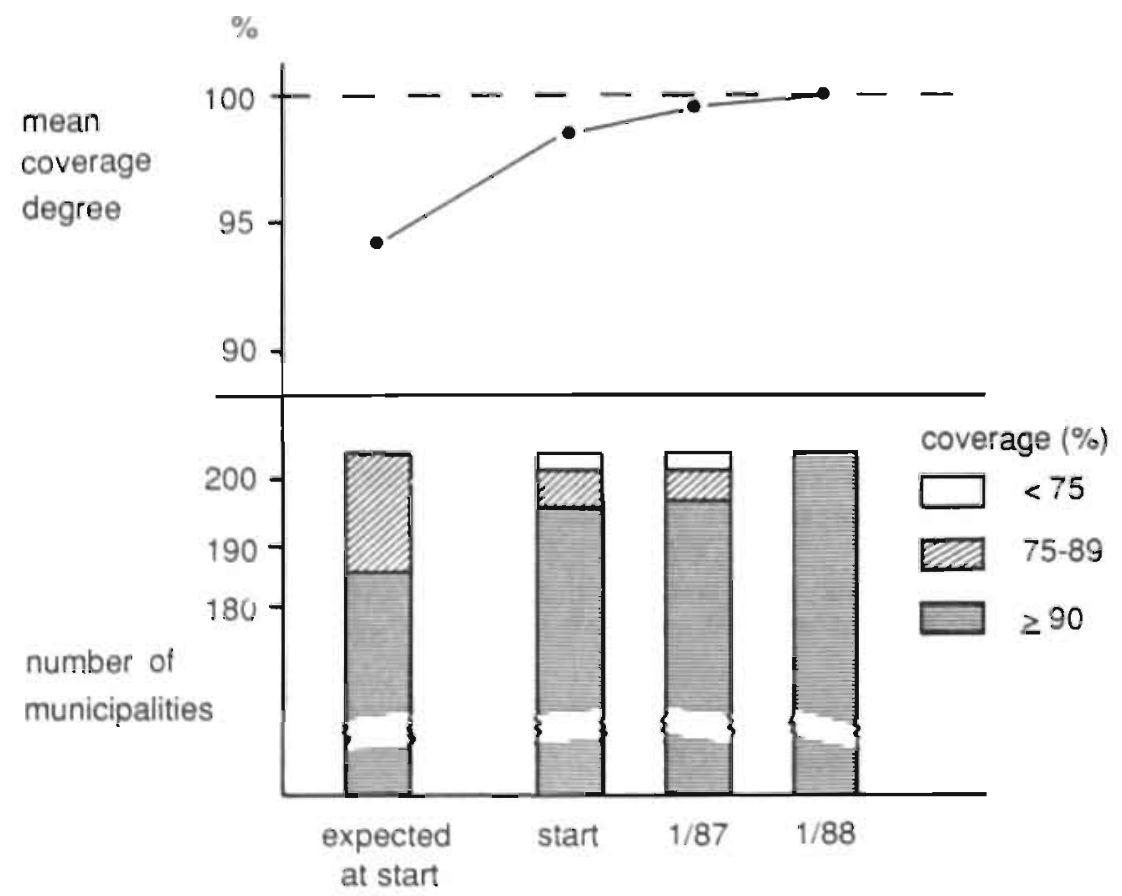

Figure 3. Anticipatedi and actual coverage of the cohort sampling population by the cancer registries and PALGA. 


\section{Discussion}

The value of the presented analysis for the evaluation of the follow-up for cancer in the cohort study depends on the reliability of the data and the underlying assumptions.

From 1986 onwards, all general and university hospitals in the Netherlands supply the required registration data to the LMR data base. As for the 1983 data base, a few hospitals were lacking. Their share in the hospital discharges was $2.5 \%$. An important hospital that was neither contributing to the 1983 nor to the 1987/1988 data base was the Daniel den Hoed Hospital, an oncology hospital at Rotterdam. For the missing hospitals, however, the most crucial data, i.e. the number of admitted patients and their residence, was known from the Enquête Jaareijfers Ziekenhuizen (EJZ, 4). The distribution of diagnoses in the missing general hospitals was estimated from that in other general hospitals. The diagnoses in the Daniel den Hoed Hospital were assumed to have the same distribution as those in the Antoni van Leeuwenhoek Hospital, the oncology hospital in Amsterdam. Thus, the overall error due to missing hospitals in the LMR data base could not have been very large.

Comparison of the LMR with the EJZ data has shown that the LMR data base is also virtually complete within each hospital (S\Gr, personal communication). However, even if incompleteness were substantial, it would not invalidate the present analysis, which is based on proportional and not absolute coverage. Some information is available on the accuracy of the recorded data: the place of residence appeared to be satisfactory recorded, but the diagnosis was not quite correct in $7 \%$ of the cases (5). Although it should have been recorded whether a patient was admitted for that specific (malignant) disease for the first time, this item is presumed to be inaccurate, in particular for university hospitals (SIG, personal communication). The analysis based on all admissions and that based on first admissions only produced similar results, however.

A substantial change over time in referral patterns, for example as a result of closing and merging of hospitals, may threaten the representativeness of the data base for the population in the cohort study. This argument is valid for the 1983 data base, which was used to estimate coverage in 1986, but not for the $1987 / 1988$ data base, which referred to exactly the same period as the follow-up of the cohort and which must actually include the cohort members diagnosed with cancer during these years of follow-up.

The results of the presented analysis are useful only, if all eligible: cases who were admitted to a hospital affiliated with a cancer registry or PALGA, were really included in these registries. A study conducted in the IKMN cancer registry, in which the 1986 cancer registry data were compared to the LMR data, has shown that $11 \%$ of the cases were initially missed by the cancer registry (6). In $52 \%$ of these missing cases, however, the diagnosis was not histologically confirmed. The percentage of cases missed was much lower for cancer of the breast (2.5) and digestive tract (6.8) than for lung cancer (12.9). These percentages reflect the proportion of cases not confirmed by histologic examination. From 1986 onwards, LMR is also introduced in most cancer registries as a check for completeness and as, additional source on cancer cases.

Another potential source of loss to follow-up would be imperfect linkage of the cohort to the records in the CR and PALGA. It has been shown that linkage with the cancer registries attains a sensitivity of $98 \%$ (7). This figure did not include the (now routinely used) adaptation to the linkage procedure which accounts for frequently occurring spelling errors in names; true sensitivity is thus likely to be somewhat higher than $98 \%$.

We conclude from the data presented here that in the cohort study loss to followup is likely to be very small. An opportunity to corroborate this conclusion was 
provided by the subcohort, a random sample of 5000 subjects from the entire cohort. Subcohort members have been followed up for vital status and have reported biennially whether they had been diagnosed with cancer after the start of the study in 1986. Of the subjects in the subcohort who reported to have a cancer (145 by the end of 1989) 115 had also been matched independently in the record linkage with the CR and PALGA. Almosit all of the missing cases (29) had reported skin cancer. This can be explained, since basal cell carcinoma of the skin is not recorded routinely by the CR. One self-reported case with another cancer type, however, was not matched to a record in the PALGA data base because of disagreement as to place of residence. After exclusion of the missed subjects reporting skin cancer, the proportion of cancer cases retrieved thus amounts to $115 / 116=99 \%$ with a $95 \%$ lower confidence bound of $96 \%$. We conclude from all evidence combined that follow-up of the cohort for cancer must be very complete.

A second conclusion concerns the use of LMR data for this type of problem. These data provided a quick, efficient and apparently reliable way to solve an important problem in the planning stage of the cohort study, i.e. how to minimize loss to followup for cancer. Although it will not be necessary to repeat this type of analysis for other (prospective) epidemiologic studies on cancer, since the CR and PALGA have attained national coverage, it may be used to check coverage by other local disease registries.

\section{References}

1. Van den Brandt PA, Goldbohm RA, van 't Veer P, Volovics A, Hermus RJ, Sturmaus F. A largescale prospective cohort study on diet and cancer in the Netherlands. J Clin Epidemiol 1990; 43: 285-295.

2. L.OK. Progress report Dutch Cancer Registry 1988. Landelijk Overieg-orgaan Kankercentra, Utrecht, 1991.

3. SIG. Onderzoek en medische registraties: een bruikbaarheidsbeoordeling in het kader van sociaaleconomische status en gezondheidsverschillen. SIG, Utrecht, 1991.

4. Enquête Jaarcijfers Ziekenhuizen. Nationaal Ziekenhuisinstituut in opdracht van de Geneeskundige Hoofdinspectie, Utrecht.

5. Hoogendoorn D. Stichting Informatiecentrum voor de gezondheidszorg (SIG). In: Epidemiologie en gezondheidsbeleid. Samson Stafleu, Aphen aan den Rijn, 1989.

6. Berkel J. Volledigheid van de kankerregistratic [Completeness of the cancer registry]. Ned Tijdschr Geneesk 1989: 133: 2027-2030.

7. Van den Brandt PA, Schouten IJ, Goldbohm RA, Dorant E, Hunen PMH. Development of a record linkage protocol for use in the Dutch Cancer Registry for epidemiological research. Int J Epidemiol 1990b: 19: 553.558. 


\title{
Development of a Record Linkage Protocol for Use in the Dutch Cancer Registry for Epidemiological Research
}

\author{
PIET A. VAN DEN BRANDT***, LEO J SCHOUTEN**, R ALEXANDRA GOLDBOHMT, ELISABETH \\ DORANT* AND PERRY M H HUNEN**
}

\begin{abstract}
Van den Brandt PA (Department of Epidemiology, University of Limburg. PO Box 616, 6200 MD Maastricht, The Netheriands), L.J Schouten, R. A Goldbohm, E Dorant and P. M. H Hunen. Development of a record linkage protocol for use by the Dutch cancet registry for epidemiological research. International Journal of Epidemiology 1990; 19: 553-558. A method has been developed to determine the optimal linkage key for record linkage between the cancer registry and a large-scale prospective cohort study in the Netherlands. The proposed linkage procedure is a two-stage process in which the initial computerized. linkage using a particular linkage key is followed by visual inspection with additional information to separate the computer matches into true and false positives. In the determination of the optimal key, both informativeness and susceptibility to ecror of personal identifiers were taken into account. The performance of the various keys in the linkage was expressed in terms of sensitivity and predictive value of a reported computer match. The key, consisting of date of birth, first four characters of the family name and gender was the optimal choice. with a sensitivity of $98 \%$ and an initial predictive value of a computer match of $98 \%$. When additional information on migration, place of birth and first initial was collected in the second stage, it was possible to eliminate the false posi. tives from the reported computer matches without loss of true positives. Thus, the sensitivity remained constant whereas the secondary predictive value of accepted matches was maximized.
\end{abstract}

In epidemiological research, the use of record linkage to disease registers for follow-up purposes is increasing. ${ }^{1.5}$ An important aspect of this kind of follow-up is the development of the linkage procedure. A general method for (medical) record linkage has been proposed $^{6}$ and then developed further. ${ }^{7.9}$ This method is based on the calculation of the odds in favour of a correct match associoted with the particular linkage key. The linkage key is the combination of personal identifiers that is used as matching variable in the computer linkage. The calculation of the: odds can be: refined in various respects to accommodate weights associated with identifier values and coding (transcription) errors. However, the method requires detailed prior knowledge about the frequency of specific identifier values. in both files that are to be matched. Usually investigators. do not have this degree of access to the disease register to which they want to link, due to confidentiality regulations. The desired frequency distributions can therefore not be determined. Also, with manual disease

-Department of Epidemiology. University of Limburg. PO Box 616. 6200 MD. Maastricht. The Netherlands

" "IKL-Cancer Registry. Comprehensive Cancer Centre Limburg. Maastricht, The Netherlands.

tDepartment of Human Nutrition. TNO-CIVO Toxicology and Nutrition Institute. Zeist, The Netherlands. registers, it is generally not feasible to determine these irequencies. ${ }^{10}$

In either of these instances, one needs to perform recond linkage based on an optimal linkage key. When a unique personal identification number is being used throughout in a country such as in Scandinavian countries, linkage with such a number is appropriate.' In other situations (which applies to many other countries, the linkage procedure should be based on a combination of identifiers serving as a key. The procedure should then be optimized with respect to the choice of identifiers in terms of their informativeness (discriminating power) versus the likelihood that they contain coding errors. This paper describes the procedure for determining the optimal linkage key to use for follow-up of a large general population cohort in the Netherlands, using cancer registries.

The uniqueness of (combinations of) identifiers has been studied in the Netherlands. "1-13 These studies were, however, theoretical in the sense that they were conducted within one dataset and did not take into account possible coding errors in identifiers that may lead to false disagreements. when two datasets are actually linked. To take account of both the informativeness and the susceptibility to error of identifiers. 
we empirically evaluated the usefulness of several keys in a linkage procedure between the cohort study and the Regional Cancer Registry Limburg (IKL-registry). Ideally, one would like to have an independent source of diagnosed cancer cases in the cohort, to check whether the matched records from the cancer registry did indeed represent all the truly diagnosed cancer cases in that cohort. There was, however, no 'gold standard" registry available in the region to which reporting of cancer patients was unrelated to reporting of cases to the IKL-registry. Instead, we evaluated the usefulness of the keys. By linkage with tolerant criteria and visual inspection of the reported computer matches, we first detected all existing correct matches between the cohort and cancer registry. After that. more strict linkage criteria were applied and the performance of various keys was tested.

\section{METHODS}

The Cohort

In 1986, a prospective cohort study on diet and cancer began in the Netherlands. The cohort $(n=120852)$ of 55-69 year old men and women originated from 204 municipal population registries. In view of the size of the cohort and the method of follow-up (record linkage to cancer registries), only computerized population registries were used. Since the identifying information from the municipalities was the most accurate information available on Dutch citizens, the recruitment procedure ensured that this quality was maintained for the cohort members. At baseline (September 1986), cohort members completed a questionnaire on diet and potential confounders. ${ }^{4}$ Relevant questions with respect to record linkage included place of birth, twinship and history of cancer. Data from the 8081 cohort members who live in the area covered by the IKLregistry were used for the present study.

\section{The IKL Cancer Registry}

The IKL eancer registry is one of the nine Dutch regional cancer registries; it has been operating since 1982 in the middle and southern part of the province of Limburg. In the period 1982-1986, cooperation was obtained from all hospitals in the area, resulting in a presumably complete coverage in 1986. "Tumour data are abstracted from pathology reports and medical records and coded according to ICD-Oncology. The identifying information is read from the patients' identity card, produced in the hospital when a patient seeks medical care. The cancer registry then converts names to a standard format (apostrophes and hyphens are replaced by spaces, the Dutch letter combination 'if' is converted into ' $y$ ' and a list of allowed prefixes is used).
After conversion, the data are enerypted before storage in the IKL-datahase. Completeness, datar consistency and the possibility of duplicate records are extensively checked by computer programs. The identifiers available for linkage in the cohort file and in the IKL-registry file are presented in Table 1.

\section{Record' Linkage}

The record linkage between the cohort $(\mathrm{n}=8081)$ and the IKL-registry was started in November 1988, after the names of the cohort members had been converted and encrypted to the cancer registry format. Malignancies registered by the IKL until 21 October 1988 $(\mathrm{n}=8917)$ were available for linkage. While for the actual follow-up of the cohort only incident cases are of interest, for the development of the linkage protocol both incident and prevalent cases were allowed to match. Prevalent cases were defined as cases diagnosed before September 1986 (ie start of the cohort study).

\section{Determination of All Existing Correct Matches}

To detect all existing correct matches between the two files, a computer linkage was carried out with very tolerant criteria for reporting a match. For this computer linkage, a program was used that had been developed earlier by the IKL to detect duplicate records in its registry. It assigns (arbitrary) scores for agreements on particular identifiers. The tolerance is adjusted by varying the total score that is needed for a match to be reported. Table 2 shows the scores that were assigned to agreements on other identifiers in this linkage exercise. No scores were assigned to agreements on other identifiers in the computer linkage. The agreement or Tamis 1 Arailable identifiers for linkage in the cohor file and in the IKL-negistry file.

\begin{tabular}{|c|c|c|}
\hline \multirow[b]{2}{*}{ Ideatifier } & \multicolumn{2}{|c|}{ Percentage availability } \\
\hline & Cohort & $\begin{array}{l}\text { IKL. } \\
\text { registry }\end{array}$ \\
\hline Date of birth & 100 & 100 \\
\hline Gender & 100 & 100 \\
\hline Family name (encrypted) & 100 & 100 \\
\hline Prefix of family name & 13 & 12 \\
\hline $\begin{array}{l}\text { Married name (surname of husband. } \\
\text { encrypted) }\end{array}$ & $87^{\circ}$ & $85^{\circ}$ \\
\hline Fint initial & 100 & 99 \\
\hline Place of birth & $100 *$ & 421 \\
\hline Residential postal code & 100 & 100 \\
\hline
\end{tabular}

*Percentage for women.

*Place of hirth is available from the cohort questionnaires, but not on file It can therefore only te used for additional visual imspection of reported computer matches. Percentage avalability is based on a random sample of 1000 questionnaires.

oDue to hosputal registration practice in the Netherlands. 
disagreement on other identifiers was, however. measured by the computer but only used for additional visual evaluation of the computer matches.

To allow reporting of a match even in the case of a considerable number of coding errors, a low threshold value for the total score was used. Various combinations of identifiers that agreed could therefore lead to a match. All matches with identical year of birth or identical first four characters of family name (F4) and a minimum score of 90 were reported. The reported computer matches were then ordered according to their scores. A match was accepted when the computer indicated agreement on: date of birth (DOB), complete family name (F), first initial (I), gender (G), postal code (P4), prefix, and married name (ie a score of 180 and agreement on the latter two identifiers). When the score was lower or other disagreements were apparent, a visual inspection of the match was performed using data on the original IKL coding forms. This was done to determine whether less than maximal agreement was due to coding errors or norn-availability of the item in one or both data files, or because it represented different subjects. Additional information on migration, birthplace, tumour site and year of diagnosis (for prevalent cases) and date of death was also used for this determination. In this way presumabiy all existing correct matches between the two data files were detected using as much information as possible.

\section{Selection of Optimal Linkage Key}

This number of correct matches was then considered the maximum number that could be obtained in subsequent linkages using other, less tolerant criteria. In these linkage exercises, (dis)agreement on identifiers

TAnte 2 Scores for agreement per identifier, used in the computer linkage.

\begin{tabular}{|c|c|c|}
\hline Identifier & Abbreviation & $\begin{array}{l}\text { Score for } \\
\text { agrecement }\end{array}$ \\
\hline \multicolumn{3}{|l|}{ Related to date of birth (DOB) } \\
\hline Year of birth & $\mathbf{Y}$ & 20 \\
\hline Month of birth & M & 20 \\
\hline Day of birth & D & 20 \\
\hline \multicolumn{3}{|l|}{ Related to name } \\
\hline Family name & $F$ & 60 \\
\hline First 4 characters of family name" & $\mathrm{F} 4$ & 40 \\
\hline First initial & 1 & 20 \\
\hline \multicolumn{3}{|l|}{ Related to address } \\
\hline Postat code (all 4 digits) & P4 & 30 \\
\hline Postal code (first 3 dipits only) & $\mathrm{P}_{3}$ & 10 \\
\hline Gender & $G$ & 10 \\
\hline
\end{tabular}

"In the Dutch cancer registry, names are first divided into two segments (F4 and the remaining characters) which are then encrypted separately. was assessed without assigning scores to it: The linkages were performed using the original datasets (ie coding errors found after the extensive linkage described above were not corrected). For each key, the number of true and false positives was calculated and the usefulness of the key was then expressed in the sensitivity (ie proportion of all correct matches obtained) and in the positive predictive value $(\mathrm{PV}+)$ of a reported match (ie proportion of reported matches that represent correct matches). The optimal key was defined as the key which shows the best combination of sensitivity and $\mathrm{PV}+$. To minimize the number of false positive matches any further, the initial computer linkage with the optimal key was followed by a second, manual stage. In this stage, matches were separated as far as possible into true and false positives, by visual checking, using additional information that was not on file (eg birthplace and migration data of cohort members). Manual collection and evaluation of these data is only feasible when the computer linkage shows a high initial PV+

\section{RESULTS}

\section{Determination of All Correct Matches}

The initial very tolerant linkage between the cohort and IKL-registry, with a threshoid score of 90 , resulted in 8499 computer matches. (In the linkage cohort members can match to more than one cancer registry record, especially with this low threshold; the maximum number of possible pairs of records is $8081 \times$ $8917=72058277$ ). Table 3 (second column) shows the distribution of computer matches according to their total scores. The maximum score that could be obtained was 180 . Computer matches with scores of $120-180$ were visuạily checked using all available identifiers, and potential migrations of cases were evaluated. All 179 computer matches with scores 160-180 were found to be correct, whereas the nine reported matches with scores of 140-150 included only one correct match. In the category with a score of 120-130, eight correct matches were found out of 185 computer matches. Al! eight records represented cases whose F4-part of the family name had not been converted by the IKL-registrars according to the standard format (ie conversion of ' $\mathrm{ij}$ ' into ' $y$ ').

With regard to computer matches with scores of 110 or less, it was anticipated that for a number of matches, the additional information would be incomplete, thereby leaving uncertainty about acceptance or rejection of the match. To overcome this problem, only matches representing prevalent cancer cases were considered first, whose diagnoses could be checked against the self-reported information on the cohort question- 


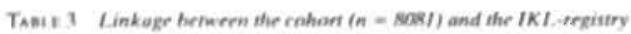
$(n=6 V 17)$, dismibution of eported computer matches according to total score, before and afier visual inspection (threshold score 90 ).

\begin{tabular}{lrrr}
\hline & \multicolumn{3}{c}{ Number of computer matches } \\
\cline { 2 - 4 } Score & Total & Fahe positives: & True positives* \\
\hline $90-110$ & 8126 & 8126 & 0 \\
$120-130$ & 185 & 177 & 8 \\
$140-150$ & 9 & 8 & 1 \\
$160-180$ & 179 & 0 & 179 \\
Total & 8490 & 8311 & 188 \\
\hline
\end{tabular}

-As determined by visual inspection of computer matches.

naire. From the computer matches with scores of $90-$ 110 , cases $(n=1059$ ) with clearly distinguishable cancer sites (eg. lung, breast, stomach, kidney), diagnosed in the year preceding September 1986 and still alive at baseline were selected from the IKL-registry. Visual assessment of the agreement between IKL-registry and cohort regarding tumour site and year of diagnosis revealed no correct matches in this group. It was therefore assumed that among the total group of matches with scores of $90-110$ no additional correct matches would be detected. This was based on the observation that among the category with scores of 120-180, all 94 correct matches representing prevalent cases with these tumour sites diagnosed in 1985 or 1986 had been self-reported by cohort members. Thus, the 8499 computef matches were separated into 188 true positives and 8311 false positives.

\section{Selection of Optimal Linkage Key}

Following the determination of presumably all correct matches, linkage with specific keys was conducted on the original datasets. The following keys were used: all identifiers iogether, individual identifiers separately and identifiers in various combinations. The results are presented in Table 4, together with the sensitivity and $\mathrm{PV}+$ (the denominator used to calculate sensitivity is 188). When all identifiers (listed in Table 1), except birthplace were used as key in the computer linkage, only 167 true positives were matched (sensitivity $88.8 \%$ ), but PV+ was $100 \%$. This clearly shows that the use of many identifiers in a computer linkage yields a considerable number of false negatives, because of an increased chance of a coding error in one or more identifiers. The 21 false negatives occurred because of errors in the IKL-file regarding $F 4(n=2)$, remainder of F (3), M (1), Y (1), I (9), married name (2), prefix (3) and $\mathbf{P} 4$ (1, ie migration). One false negative was the result of simultaneous errors in 1 and in the married name. Excluding DOB from this key resulted in a substantial decrease of $\mathrm{PV}+$ with only a minor improvement of sensitivity.
To identify the optimal linkage key while striving for parsimony, we started with identifiers that have been indicated in the literature to be relevant: ${ }^{12,13} \mathrm{DOB}, \mathrm{F}$ or F4, G. Using DOB as the only identifier in the linkage key resulted in a very high sensitivity $(98.9 \%)$, accompanied' by many false positives (PV $+3.5 \%$ ). Using only F or F4 resulted in many more computer matches, indicating that the family name is far less informative than DOB. With F4, an increased sensitivity was noted compared to $\mathrm{F}$. Combining $\mathrm{DOB}$ and $\mathrm{F} 4$ resulted in a $\mathrm{PV}+$ of $94.8 \%$ with a sensitivity of $97.9 \%$. The combination DOB/F $4 / \mathrm{G}$ showed a high sensitivity $(97.9 \%)$ as well as a high $\mathrm{PV}+(97.9 \%)$. Extending this key with $\mathrm{I}$, P4 or P3 elevated PV + further, but reduced sensitivity at the same time. Moreover, the use of postal codes in any key is not feasible because theit value is timedependent (migration is-not assessed continuously for all cohort members). The keys. DOB/G and $F 4 / G$ are not attractive options: $\mathrm{PV}+$ is very low, although sensitivity is high $(98.9 \%)$. The key DOB/F $4 / G$ was therefore considered optimal, given the combination of its sensitivity and its PV + . With the use of this key, 188 computer matches were reported. Using additional informacion on $\mathrm{P} 4$ and birthplace, it was possible to separate these 188 matches correctly into true and false positives, and maintaining the same level of sensitivity. (When place of birth was unknown, agreemen? on other identifiers had to exist to accept a match). Also,

TAse 4 Linkage berween the cohort $(n=808 I)$ and the IKL-registry $(n=8917)$; number of reported computer matches, true positives; false negatives, sensitiviry and PV+ obtained' with various linkage keys, consisting of all identifiers together, individual identifiers separately or combinations of identifiers.

\begin{tabular}{|c|c|c|c|c|c|}
\hline Key & Matches & $\begin{array}{l}\text { True } \\
\text { positives }\end{array}$ & $\begin{array}{c}\text { False } \\
\text { negatives }\end{array}$ & $\begin{array}{c}\text { Sensitivity } \\
(\%)\end{array}$ & $\begin{array}{l}\mathrm{PV}+ \\
(\%)\end{array}$ \\
\hline All identifiers & 167 & $16 ?$ & 21 & 88.8 & 100 \\
\hline Ali, except DOB & 197 & 169 & 19 & 89.9 & 85.8 \\
\hline $\mathrm{DOB}$ & $\$ 276$ & 186 & 2 & 98.9 & 3.5 \\
\hline $\mathrm{F}_{4}$ & 102.070 & 186 & 2 & 98.9 & 0.2 \\
\hline F & 49808 & 183 & 5 & 97.3 & 0.4 \\
\hline $\mathrm{DOB} / \mathrm{G}$ & 2664 & 186 & 2 & 98.9 & 7.0 \\
\hline $\mathrm{DOB} / \mathrm{F}^{4}$ & 194 & 184 & 4 & 97.9 & 94.8 \\
\hline $\mathrm{F} 4 / \mathrm{G}$ & 50970 & 186 & 2 & 98.9 & 0.4 \\
\hline DOB/FA/G & 188 & 184 & 4 & 97.9 & 97.9 \\
\hline DOB/F/G & 184 & 181 & 7 & 96.3 & 98.4 \\
\hline $\mathrm{DOB} / \mathrm{G} / \mathrm{P} 4$ & 203 & 185 & 3 & 98.4 & 91.1 \\
\hline DOB/G/P3 & 321 & 185 & 3 & 98.4 & $\$ 7.6$ \\
\hline $\mathrm{DOB} / \mathrm{F} 4 / \mathrm{G} / 1$ & 175 & 175 & 13 & 93.1 & 100.0 \\
\hline DOB/F4/F/P: & 183 & 183 & 5 & 97.3 & 100.0 \\
\hline DOB/F4/F3 & 184 & 183 & 5 & 97.3 & 99.5 \\
\hline DOB/F4/G/L3 & 174 & 174 & 14 & 92.6 & 100.0 \\
\hline DOB/F/G/1 & 172 & 172 & 16 & 91.5 & 100.0 \\
\hline
\end{tabular}

- Multiple matches possible per cohort member, especially when the linkage key consists of few identifiers. 
linkage with the key $\mathrm{DOB} / \mathrm{F} 4 / \mathrm{G}$ resulted in four false negatives. This was due to misspellings in $F 4(n=2)$ and errors in M (month of birth) (1) and $\mathrm{Y}$ (year of birth) (I).

\section{DISCUSSION}

We evaluated the usefulness of various keys for record linkage between two datasets. In general, one would prefer to use as many identifiers as possible for accurate discrimination between individuals, especially when very large files are being used. This, does not imply that one should use all identifiers in a linkage key, because identifiers may also contain errors. The optimal choice of identifiers to be included in the key depends on their informativeness versus susceptibility to error and on the time-dependency of identifier values. Thus, a record linkage using all identifiers is not a very sensitive method, although the predictive value of such a reported computer match may be maximal. The sensitivity is increased by using less identifiers in the key, but this produces more false positive matches. The problem can be dealt with when the available identifiers are used in two steps. Firstly, one uses a relatively small number of accurate identifiers in the initial computer linkage. Secondly, true and false positives are separated in a visual check using other information which is highly informative and accurate, but may require: additional collection. Given the extremely high specificity values for all reported keys, the optimal key is then the key with the highest sensitivity given a reasonably high $\mathrm{PV}+$ (ie a number of false positives that can reasonably be identified manually).

The key DOB/F $4 / G$ behaved optimally in this respect. Extending the number of characters of $F$ beyond four adds only a small amount of iniormation. ${ }^{3}$ Also, errors tended to occur more towards the end of the name. The resuits indicate that the first initial is especially prone to coding errors. This is partly due to discrepancies between given and (municipal) Christian names that can exist for Dutch subjects. For example, frequently occurring given names as 'Hans' or 'Kees' have Christian names 'Johannes' and 'Cornelis', respectively. In separating true and false positives, information on birthplace and (for migration corrected) postal code was very important. Unfortunately, the informative ${ }^{13}$ and accurateiy recorded" birthplace is not commonly registered in Dutch hospitals.

The score values used in the initial determination of all correct matches are somewhat arbitrary, although they roughly reflect the informativeness of the items. ${ }^{13}$ However, the score values per se are not important in this respect, as long as one is able to separate matches correctly. The use of weights in record linkage com bined with manual verification has been described before, 16,17

Due to coding errors, the key DOB/F4/G was associated with a false negative rate of $2 \%$. By anticipating certain potential errors in $\mathrm{F} 4$ and $\mathrm{DOB}$, one can potentially increase the sensitivity to some extent. Wich regard to names, various phonetic coding systems have been developed in England, Canada and the US to match variant spellings of names. ${ }^{7.18 .10}$ Such a sysiem is not available for the Netherlands and it cannot be applied to the cancer registries because these contain encrypted names. Instead, the data of cohort members are now systematically scrutinized for name types that can casily be misspelled when the subject is registered in a hospital. In future linkages, an additional (flagged) record will be used, containing the misspelled version of the name. When a match occurs on this additional record, it will be carefully evaluated in the second stage of the linkage process in order to avoid an increase in the number of accepted false-positive matches. The two errors in DOB-items were of the type $\mathrm{M} \pm \mathrm{I}$ and $\mathrm{Y} \pm 1$. Analyses of duplicate records in the IKL-registry indicated that more than half of the coding errors in DOB-items consisted of two sorts: value \pm 1 and \pm 10 . Such error patterns can also be anticipated on in future linkages. ${ }^{19}$

For the cohort study, separate linkages with all regional cancer registries are planned, in which the specific regional cohorts will be selected from the large cohort and linked to the respective registries. In this way, the prevalence of correct matches in these linkages will be comparable to the present study, as will be PV+.

\section{APPENDIX}

DOB $=$ date of birth; $D=$ day of birth $\bar{F}=$ family name (excluding prefix); $\mathrm{F} 4 \mathrm{f}=$ first four characters of family name; $G=$ gender $I=$ first initial; $M=$ month of birth; $\mathbf{P} 4=$ postal code (all four digits); $\mathbf{P} 3=$ postal code (first three digits only); $\mathrm{PV}+=$ prediceive value of a positive match; $\mathrm{Y}=$ year of birth.

\section{ACKNGWLEDGEMENTS}

This study was supported in part by the Netheriands Cancer Foundation and the Ministry of Welfare, Public Health and Cultural Affairs.

The authors thank Gregor Franssen, Ruud Schmeitz, Marielle Bethlehem and. Patricia Florax for their assistance in the conduct of this study.

\section{REFERENCES}

'Storm H H. Completeness of cancer registration in Denmark 19431966 and efficacy of record linkage procedures. Int I E pidemiol 1988; 17: 44-9 
'Wesierholm P. Ahlmark A. Maasing R, Segelberg I Silicosis and nok of lang cancer or lung tuberculonis; a cohort stady Emi.

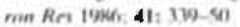

'Stampfer M J. Willen W C. Speizer F E. Dysent D C, Lipnick R. Resnef B, Hennekens CH. Test of the National Death Index Am J Epidemiol 19m4; 119: 837-9.

'Howe G R. Lindsay J' A generalized iterative record linkage computer system for use in medical follow-up studies. Comp Bio med Res 1981: I4: $327-40$

'Goldacre M, Hawion $\mathrm{X}$. Repetition of self-poisoning and subsequent death in adolescents who take overdoses. Br J Psychi. airy 1985 ; 146 i $395-8$

"Newcombe HA B, Kennedy J M., Axford S J, James A P. Automatic linkage of vital records. Science 1959; 130: 954-9.

'Acheson E D. Medical Recond Linkage. London: Oxford University Press, 1967

'Baldwin J A. Acheson E D, Graham W J, eds. Textbook of medical mord linkage. Oxford Oxford Univenuty Press, 1987.

"Newcombe H Bi. Handbook of Recond Linkage: merhods for health and statisical studies, administration and busimess. Oxford: Oxford University Press, 1988

=Fett M J. The development of matching criteria for epidemiological studies using record linkage techniques. Int / Epidemiol 1984; 13: $351-5$

"Hoogendoorn D. Medical record linkage; de identificatie van de patient. Ned Tjuduchr Generste 1973; 117: 1416-23. s Sciverling R. Hoedemacker Ph J. Administratieve identificatie van paticaten in grote bevolkingseroepen. Ned Tijdschr Genceskd 1975: 119: 1272-7?

"Van 't Hol-Grootenboer A E. Verbeek A L. M. Mogelijkheden tot administratieve identificatic in bevolkingsonderzock. Tijd. schriff Sociale Gezondheidszorg 1986; 64: 586-90.

" Van den Brandi P. A. Goldbohm. R A. Van ' Veer P. Hermus R J J. Sturmans F. Dietary habits and the aetiology of cancer. Int J Epidemiol 1988; 17: 472 .

"Schouten L. J, Van den Brandt P. A. Jager J J. Regional Cancer Regis. tration IKL. Cancer Incidence /986. Maastricht, the Nether. lands: Comprehensive Cancer Centre Limburg. 1989.

" Goldacre M J. Clarke J A. Heasman M A. Vessey M P. Follow-up of vasectomy using medical record. linkage. Am J Epidemiol 1978: 108: 176-80

"Baldwin I A. Gill L. E. The district number: a comparative test of some record matching methods. Comm Medicine 1982; 4: 265-75.

"Baldwin J A. Medical Record Linkage. Medicine 1974; $35: 2048-50$.

"Patterson B H. Bilgrad R. Use of the National Death Index in cancer studies: JNCI 1986; $77: 877-81$.

(Revised version received February I990), 


\title{
Chapter 5
}

\section{Validation of a dietary questionnaire used in a large-scale prospective cohort study on diet and cancer*}

\author{
R. Alexandra Goldbohm ${ }^{1.2}$, Piet A. van den Brandt', Henny A.M. Brants ${ }^{1}$, \\ Pieter van 't Veer', Monique $\mathrm{Al}^{3}$, Ferd Sturmans', Rudolph J.J. Hermus'. \\ 1) TNO Toxicology and Nutrition Institute, Zeist, the Netherlands; 2) University of Limburg, Department of \\ Epidemiology, Maastricht, the Netherlands; 3) Universiry of Limburg, Department of Human Biology, \\ Maastricht, the Netherlands
}

\section{Abstract}

The validity of a self-administered malled dietary questlonnaire (150 food items), used in a cohort study on diet and cancer (120,852 men and women, aged 55*69), was investigated in a subgroup of the cohort ( 59 men and 50 women) two years after the baseline questionnaire was completed. A dletary record, kept over three 3-day periods, four to five months apart, served as reference method. Pearson correlation coemelents between nutrient intakes assessed by the record and the questlonnalre that was completed afterwards ranged from 0.40 for vitamin B-1 to 0.86 for alcohol Intake, with correlations for most nutrients between 0.6 and 0.8 . Adjustment for energy Intake and sex did not materially affect these correlations, except the correlation for fat Intake, which changed from 0.72 to 0.52 .

To evaluate the representativeness of the study population for the entIre cohort, a comparison was made with the baseline questlonnaire of a random sample of the cohort. Correlation coefficients were only slightly modifled when the results were extrapolated to the cohort at large. Correction of correlation coefnclents for attenuation by day-to-day variance in the record data improved them by 0.07 on average. It is concluded that the questlonnalre is able to rank subjects according to intake of food groups and nutrients. 


\section{Introduction}

A self-administered dietary questionnaire is often the method of choice in a largescale epidemiologic study, such as a prospective cohort study, into dietary habits and disease. The validity of such a questionnaire is not self-evident, since it is limited with respect to the foods included and the degree to which portion sizes are quantified. Moreover, each questionnaire needs to be tuned to the specific dietary habits of the study population. Validation studies of a number of self-administered dietary questionnaires have been published (e.g. 1-9).

We developed a self-administered, mailed dietary questionnaire for use in a largescale prospective cohort study on dietary habits and cancer in the Netherlands (10). The cohort, consisting of 120,852 men and women aged 55-69, was recruited from the: general population and completed the baseline questionnaire in 1986 (11). The: questionnaire is repeated each year in random samples of the cohort $(n=400)$ to assess its reproducibility and the stability of dietary habits over time.

This paper describes the validity of the dietary questionnaire as compared to a nineday diet record. Considering, the etiologic purpose of the cohort study, validity of the questionnaire is primarily defined as its ability to rank study subjects according to nutrient intake and food (group) consumption. Since the performance of a questionnaire also depends on the actual study population, the validation study was conducted within the cohort. Assessment of selection bias, potentially introduced by incomplete participation in the validation study, was included in the study design.

\section{Materials and methods}

\section{Study design}

The diet record method was used as reference method, since its errors are assumed to be independent of the errors in a food-frequency type questionnaire (12). Dietary intake wass recorded over three periods (of three consecutive days each, Figure 1), representing three seasons in the Netherlands differing with respect to consumption patterns for (specific) vegetables, fruits and meat $(13,14)$.

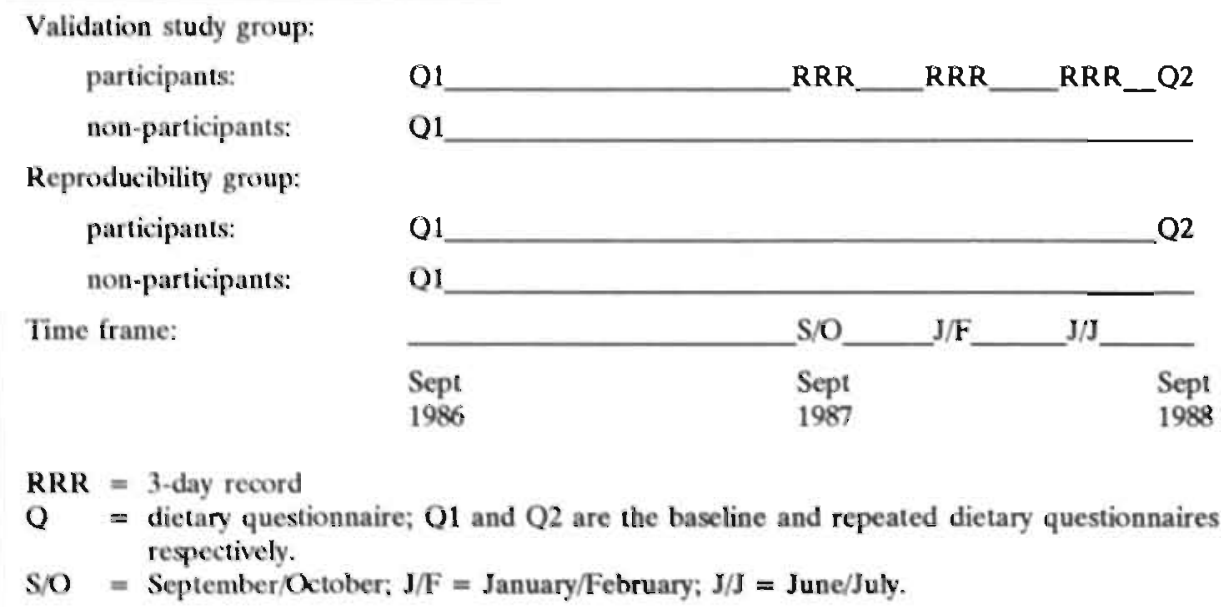

Figure 1. Design of the validation study of the dietary questionnaire used in the Cohort Study on Diet and Cancer in the Netherlands, 1987/1988. 
The nine recording days were balanced across the days of the week for each subject and for the study group as a whole. The diet record was compared to the questionnaire that was completed approximately three months after the last recording period.

To investigate a possible learning effect of recording of intake, the questionnaire application coincided with the repeated questionnaire completed annually in the cohort by participants of the aforementioned reproducibility study (Figure 1). Furthermore, to assess the representativeness of the validation study group (participants as well as nonparticipants) with regard to the cohort, the baseline questionnaires of this group were compared to those of the 1988 reproducibility study group, which constituted a random sample of the cohort.

Unless specified otherwise, all results pertain to the questionnaire completed by the study subjects at the end of the year in which recording took place.

\section{Subjects}

Since the participants had to be visited at home during each recording period, recruitment was confined to 12 municipalities, located in an eastern and a western region of the Netherlands. As far as degree of urbanization was concerned, these municipalities were representative of the 204 from which the cohort was recruited.

Of a total of 212 randomly selected cohort members (107 men and 105 women), 109 subjects ( 59 men and 50 women) completted the validation study ( 51 percent); 92 did not participate from the start, and 11 dropped out during the study. Reasons for non-participation and drop-out could be attributed to refusal (two thirds) and unavailability (death, no contact, absence during one or more recording periods Among the non-participants, six subjects were excluded because they did not manage to keep the record or did not eat at home most of the time and were hence not expected to keep a good record.

\section{Dietary questionnaire}

The purpose of the dietary questionnaire was to assess habitual consumption of approximately 150 foods during the past year. The foods included in the questionnaire were originally selected according to their contribution to the between-person variance of the intake of energy and of the following nutrients: protein (vegetable as well as animal), fat (saturated, monounsaturated and polyunsaturated), cholesterol, carbohydrates (mono- and disaccharides, polysaccharides), dietary fiber (of cerea! as well as vegetable and fruit origin), alcohol, calcium, vitamin $A$, B-carotene and vitamin $C$ (10). The contribution to the variance was calculated from a data set previously collected by means of a dietary history method in a population of men and women of the same age category as the cohort.

For each item, the questionnaire asked for frequency of use on a scale of seven frequency categories: never/less than once per month, once per month, 2-3 times per month, once per week, 2-3 times per week, 4-5 times per week, 6-7 times per week. The number of servings per consumption frequency was asked in natural (e.g. apple, slice of bread) or household units (e.g. glass, spoon). For cooked vegetables and meat, the typical individual serving size in grams was asked. For several items, the frequency categories were replaced with the number of serving units taken daily (coffee, tea, bread), weekly (eggs, onions, tomatoes) or monthly (mushrooms, sweet peppers). Ouestions on vegetables were specified with respect to season (summer and winter). Margarine used on bread and cooking fats and oils were specified as to type and brand in open questions. An open-ended question also asked to list any foods eaten regularly (once a week or more) but not included in the questionnaire. 
Questionnaires were double-keyed and automatically coded by the data-entry program. Data were checked for completeness, consistency, range, and other response errors and corrected whenever feasible by means of an SPSS computer program, which had been developed using the data from the first 3000 cohort questionnaires entered and from an earlier small validation study (15). The resulting program ensures identical cleaning procedures for all questionnaires.

To determine the completeness and the quality of the questionnaires, they were evaluated by means of the number of blank items and by means of an error index, which was calculated as the sum of the scores of 15 variables that indicated each the presence of a specific response error (see footnote to Table 4). Visual inspection of the cohort questionnaires had revealed that part of the subjects had consistently skipped items that they never ate, instead of checking the frequency category "never/less than once per month". Questionnaires were considered unacceptably incomplete when either: (a) more than 60 items (out of 150) were left blank and less than 35 items were eaten at least once a month; or (b) one or more item blocks (i.e. groupings of items in the questionnaire, e.g. beverages) were left blank. According to these criteria 6.0 percent of the cohort (6.5 and 5.5 percent for men and women respectively), among whom 1 percent had mistakenly skipped a page, has to be excluded from etiologic analyses relating dietary habits to cancer. In addition, 1.0 percent of the cohort members were excluded because the error index of their questionnaires exceeded 10 . This criterion was based on the subjective verdict of inconsistency after visual inspection of the questionnaires.

\section{Diet records}

The diet records were collected and coded by nine (student) dietitians (three for each recording period), who were trained and supervised by one experienced dietitian (H.A.M.B.), who also checked the coding of each record. The participants were asked to write down all foods and beverages taken and to specify type and brand. The amount had to be specified in their own household measures (glass, etc.) and/or weight as purchased. We did not use a weighed record method since, in our experience with untrained subjects, it is liable to mistakes. Moreover, it has been found that weighing could influence eating habits (16).

One day before the beginning of the recording period, the participant was instructed at home by the dietitian and received the diary, including written instructions and examples. The day after the last recording day, the same dietitian checked the diary with the subject and, if necessary, with the subject's partner. During the same visit, the dietitian measured the capacity of the household utensils (glasses, cups, etc.) specified in the diary and weighed the amount of butter or margarine used on bread and the amount of sugar used in tea and coffee. For the second and third recording periods, the instruction visit, but not the check visit, was skipped and diaries were mailed to those participants who appeared to have properly understood the record-keeping procedure.

\section{Calculation of intake of nutrients and food groups}

Mean individual nutrient intake per day was calculated from the record as the average of the nine recording days. Questionnaire data were converted to mean daily intake by multiplying consumption frequency, number of serving units and weight of a unit (either standard or individual). The weight of a standard serving was either derived from pilot study data or from common Dutch household measures. If the number of serving units was omitted, the median number found among other questionnaires was taken instead. Since the serving sizes of potatoes and other bulk foods, such as rice and pasta, appeared to be proportionally related within subjects, the substituted number of 
serving units for these bulk foods was derived from the serving size of potatoes for the same subject. Season was taken into account when applicable.

Record and questionnaire data (mean daily item intake) were both converted to nutrient intake using the computerized Dutch food composition table (17). Although validation of supplement use was included in the study design, nutrient intake through supplements is not taken into account in this paper. Results indicated that vitamin supplements (A, C or multivitamin supplements) were used by 3 to 9 percent of the validation study population and correctly reported by 67 percent of the users; calcium supplements were correctly reported by 53 percent of the fifteen (14 percent) users (Dorant et al., submitted for publication).

The items in the questionnaire were also aggregated into 27 food groups according to their shared properties and origin (e.g. bread, vegetables). For each food group mean daily weight consumed was calculated. The purpose of classification was to evaluate the validity of the questionnaire with respect to food group-related properties other than the nutrients studied and to facilitate interpretation of the strengths and limitations of the questionnaire.

\section{Data analysis}

Nutrient intakes calculated from the record and the questionnaire were $\log _{e}$-transformed to improve their distribution towards normality. Results were, however, similar for untransformed data. An alcohol intake of 0 gram per day was replaced with 0.1 gram per day before transformation. Energy-adjusted nutrient intakes were calculated as residuals from regression of each loge (nutrient) on loge (energy) and sex (12). Pearson correlation coefficients between record and questionnaire were calculated for unadjusted and adjusted nutrient intakes,

Furthermore, men and women were divided into quintiles according to nutrient intake (unadjusted and energy-adjusted) assessed by the questionnaire. For each quintile, the corresponding mean (untransformed) nutrient intake as assessed from the record was calculated (12). For this procedure, energy-adjusted residuals, to which mean nutrient intake was added, were calculated from untransformed energy and nutrient intakes.

For the comparison regarding the 27 food groups, most of which had a skewed distribution, a Spearman correlation coefficient was used. The specific food items within each food group were not analyzed individually, because estimation of their usual consumption frequency on the basis of a nine-day record was expected to be imprecise.

Analysis of variance was applied to both the number of blank items and the error index of the baseline questionnaire ( $\log _{e}$-transformed), assessing the effects of group (validation study group versus reproducibility study group) and participation status for the repeated questionnaire (participants versus non-participants). The presence of a learning effect with respect to the number of blank items and the error index (repeated versus baseline questionnaire) was investigated in both study groups with a paired $\mathrm{t}$-test.

To account for possible differences in the error index among the validation subgroup and the cohort, Pearson correlation coefficients were adjusted to the distribution of the error index in the baseline questionnaires of the reproducibility sample. Calculations were performed using the error index dichotomized at the highest tertile (scores five and over) in the cohort. Regression analyses of nutrient intake assessed by record on that assessed by questionnaire were conducted within each of the two groups that differed with respect to error index. In the usual formula for a squared correlation coefficient the residual sum of squares in the numerator was replaced with the residual sum of squares within both groups together with the sum of squares of 
regression over both groups. Thus, correlation coefficients can be calculated according to:

$$
\mathrm{B}^{2}=\mathbf{1}-\left[\sum_{\mathrm{i}=1}^{2}\left(\mathrm{df}_{\mathrm{i}} \times \mathrm{RMS}_{\mathrm{i}}\right)+\mathrm{SS}\right] / \mathrm{SSY}
$$

In this formula, $\underline{i}$ denotes the group, df the degrees of freedom, RMS the residual mean square of regression within group i, SS the sum of squares of the regression over groups and SSY the variance of the dependent variable (i.e. nutrient intake assessed by record). The actual adjustment for the error index was conducted by substituting the degrees of freedom in both groups for those derived from the distribution of the error index in the reproducibility sample.

Finally, because day-to-day variation will still have influenced the observed mean individual intake based on nine days (18), correlation coefficients were adjusted for this source of variation according to Beaton et al. (19) with 95 percent confidence intervals according to Rosner and Willett (20). For this purpose "the ratios of within-subject to between-subject variance of nutrient intake were calculated from the nine recording days, ignoring day-of-the-week and period effects.

\section{Results}

Out of a total of 109 questionnaires completed by the validation study subjects, 2 (1.8 percent) were incomplete according to the formal criteria, leaving 107 questionnaires (59 from men and 48 from women) for analysis. The corresponding percentage for the reproducibility study group was 4.7 . No questionnaires needed to be excluded for an error index exceeding 10.

Table 1 presents the mean daily nutrient intake for both dietary methods as well as unadjusted and adjusted (for energy and sex) Pearson correlation coefficients. Data for men and women were pooled since none of the correlations differed significantly between men and women. For most nutrients mean intake according to the questionnaire was lower than according to the record; only for polyunsaturated fat, dietary fiber, niacin and vitamin $\mathrm{C}$ the questionnaire gave a higher intake. On average, the questionnaire covered 91 percent of the record intake. Unadjusted correlation coefficients ranged from 0.40 ( 95 percent confidence interval (CI) $0.22-0.54$ ) for vitamin B-1 to 0.86 (CI 0.80-0.90) for alcohol, with a median of 0.69 .

The only substantial (though statistically non-significant) differences in correlations between men and women were found for dietary fiber ( 0.79 and 0.63 respectively). vitamin $A(0.58$ and 0.46$)$ and vitamin B-2 (0.66 and 0.55$)$. For fiber the sex difference was attributable to the higher range in intake of bread for men, for vitamin B-2 one woman had an outlying residual that was responsible for the lower correlation. Correlation coefficients adjusted for energy intake and sex ranged from 0.33 (CI $0.15-0.49$ ) for vitamin B-1 to 0.86 (Cl 0.80-0.90) for alcohol, with a median of 0.67 . Spearman correlation coefficients, calculated from untransformed nutrient intakes, are included in Table 1 for the purpose of comparison. They were slightly lower than the corresponding Pearson correlation coefficients.

To assess the relation between record and questionnaire data for different reference periods, the baseline questionnaire was also compared to the record (Table 1). Energyand sex-adjusted correlation coefficients for the baseline questionnaire ranged from 0.25 (CI 0.06-0.43) for vitamin A to 0.85 (CI 0.79-0.89) for alcohol, with a median of 0.64. No systematic differences were found between the baseline and repeated questionnaires with respect to absolute intake of energy and nutrients (data not shown). 
Table 1. Mean daily energy and nutrient intake as assessed by 9-day record and by the questionnaire, and Pearson correlation coefficients" between the two methods ( 59 men, 48 women): Cohort Study on Diet and Cancer in the Netherlands, 1987/1988.

\begin{tabular}{lllll} 
Nutrient & Record & Questionnaire & $\begin{array}{l}\text { Record with } \\
\text { questionnaire }\end{array}$ & $\begin{array}{l}\text { Record with } \\
\text { baseline } \\
\text { questionnaire }\end{array}$ \\
\hline Mean SD & Mean (\%)† SD & Unadjusted $\mathrm{r}$ Adjusted $\mathrm{r}$ Adjusted $\mathrm{r}$
\end{tabular}

\begin{tabular}{|c|c|c|c|c|c|c|c|c|c|}
\hline Energy (kcal) & 2219 & 445 & 1898 & ( 86$)$ & 477 & 0.74 & $(0.70)$ & & \\
\hline Total protein (g) & 77.5 & 15.2 & 68.6 & ( 88$)$ & 13.9 & 0.61 & $(0.54)$ & $0.59(0.52)$ & 0.61 \\
\hline Vegetable protein (g) & 24.5 & 6.0 & 24.6 & (100) & 7.2 & 0.77 & $(0.73)$ & $0.68(0.67)$ & 0.71 \\
\hline Animal protein (g) & 53.1 & 14.0 & 44.0 & ( 83$)$ & 10.7 & 0.61 & & $0.64(0.58)$ & 0.69 \\
\hline Total fat $(\mathrm{g})$ & 100.2 & 27.1 & 82.5 & ( 82$)$ & 26.5 & 0.72 & & $52(0.50)$ & 0.47 \\
\hline Saturated (S) fat (g) & 42.2 & 12.5 & 32.4 & ( 76$)$ & 10.9 & 0.73 & $(0.73)$ & $0.58(0.53)$ & 0.59 \\
\hline Polyunsaturated $(\mathrm{P})$ fat $(\mathrm{g})$ & 17.0 & 7.1 & 17.8 & (105) & 9.1 & 0.73 & $(0.70)$ & $0.75(0.73)$ & 0.63 \\
\hline P/S ratio & 0.42 & 0.19 & 0.58 & & 0.28 & 0.76 & $(0.79)$ & $0.76(0.77)$ & 0.66 \\
\hline Cholesterol (mg) & 330 & 99 & 243 & ( 74$)$ & 76 & 0.66 & $(0.71)$ & $0.62(0.65)$ & 0.64 \\
\hline Total carbohydrates (g) & 227.5 & 51.2 & 200.8 & ( 88$)$ & 57.9 & 0.77 & $(0.72)$ & $0.71(0.65)$ & 0.71 \\
\hline $\operatorname{des}(\mathrm{g})$ & 111.9 & 32.6 & 92.5 & ( 83$)$ & 37.0 & 0.78 & $(0.7)$ & $0.79(0.77)$ & 0.68 \\
\hline Polysa & 115.6 & 32.4 & 108.3 & ( 94$)$ & 34.1 & 0.83 & $(0$. & $0.79(0.7$ & 0.79 \\
\hline Dieta & 25.7 & 6.8 & 27.3 & (106) & 7.7 & 0.74 & $(0.68)$ & $0.74(0.68)$ & 0.70 \\
\hline \multicolumn{10}{|l|}{ Alcohol (g } \\
\hline ticipants & 13.2 & 14.7 & 10.7 & ( 81$)$ & 12.1 & 0.86 & $(0.89)$ & $0.86(0.88)$ & 0.85 \\
\hline only§ & 16.3 & 14.8 & 133 & $(86)$ & 12.1 & 0.78 & $(0.85)$ & $0.76(0.82)$ & 0.74 \\
\hline Wate & 2281 & 508 & 2140 & ( 94 ) & 484 & 0.73 & $(0.73)$ & $0.75(0.74)$ & 0.67 \\
\hline Calcium (mg) & 1076 & 332 & 908 & ( 84$)$ & 268 & 0.60 & $(0.55)$ & $0.62(0.54)$ & 0.62 \\
\hline Phosphorus (mg) & 1545 & 345 & 1402 & (91) & 317 & 0.66 & $(0.58)$ & $0.69(0.67)$ & 0.60 \\
\hline Potassil & 3654 & 637 & 3551 & ( 97$)$ & 695 & 0.66 & $(0.63)$ & $0.71(0.67)$ & 0.62 \\
\hline (mg eq. 1) & 0.95 & 0.32 & 0.87 & ( 92$)$ & 0.29 & 0.52 & $(0.49)$ & $0.48(0.44)$ & 0.25 \\
\hline Vitamin $\mathrm{I}$ & 1.13 & 0.24 & 1.09 & $(96)$ & 0.25 & 0.40 & $(0.42)$ & $0.33(0.37)$ & 0.57 \\
\hline Vitamin B-2 (mg) & 1.69 & 0.46 & 1.47 & ( 87$)$ & 0.38 & 0.62 & & $0.67(0.62)$ & 0.72 \\
\hline 8 & 1447 & 294 & 1416 & (98) & 322 & 0.67 & $(0.65)$ & $0.67(0.62)$ & 0.61 \\
\hline Niac & 13.3 & 4.3 & 13.6 & (102) & 3.9 & 0.62 & $(0.61)$ & $0.61(0.64)$ & 0.67 \\
\hline$C(\mathrm{mg})$ & 96.7 & 42.4 & 104.4 & (108) & 39.4 & 0.58 & $(0.52)$ & $0.55(0.51)$ & 0.42 \\
\hline Iron (mg) & 12.8 & 2.7 & 12.4 & ( 97$)$ & 2.7 & 0.58 & $(0.61)$ & $0.53(0.54)$ & 0.48 \\
\hline Protein, \% of energy intake & 14.2 & 2.5 & 14.7 & & 2.4 & 0.67 & $(0.62)$ & $0.59(0.58)$ & $0.6]$ \\
\hline Fat, \% of energy intake & 40.3 & 5.0 & 38.7 & & 5.6 & 0.57 & $(0.56)$ & $0.52(0.50)$ & 0.47 \\
\hline $\begin{array}{c}\text { Carbohydrates, } \% \text { of energy } \\
\text { intake }\end{array}$ & 41.3 & 5.5 & $\$ 2.4$ & & 5.9 & 0.72 & $(0.68)$ & $0.71(0.68)$ & 0.71 \\
\hline
\end{tabular}

\footnotetext{
- Based on $\log _{\text {- }}$-transformed values. In parenthesis: Spearman correlation coefficients for untransformed data.

$\uparrow \%$ of record mean.

\$: Adjusted for energy intake and sex.

$\S \mathrm{n}=86$, based on alcohol users according to questionnaire.

I Includes water in beverages and foods.

Img equivalents: retinol (mg) $+B$-carotene $(\mathrm{mg}) / 6$.
}

Table 2 shows the intake of food groups and the correlation between the two methods. As for the nutrients, the questionnaire generally resulted in lower intakes than the record; exceptions were vegetables, citrus fruits, bread and added fats. On average, the mean of the intakes of all food groups as assessed by questionnaire accounted for 
85 percent of the record assessment. The Spearman correlation coefficients ranged from 0.38 for vegetables to 0.89 for alcoholic beverages, with a median of 0.60 .

Table 2. Mean daily intake of food groups (g) as assessed by 9-day record and by the questionnaire, including correlation coefficients (59 men, 48. women): Cohorl Study on Diet and Cancer in the Netherlands, 1987/1988.

\begin{tabular}{|c|c|c|c|c|c|c|}
\hline \multirow[t]{2}{*}{ Food group } & \multicolumn{2}{|c|}{ Record } & \multicolumn{3}{|c|}{ Questionnaire } & \multirow[b]{2}{*}{ Spearman's $r$} \\
\hline & Mean & $\mathrm{SD}$ & Mean & $(\%) \dagger$ & SD & \\
\hline Potatoes & 162 & 83 & 136 & ( 84$)$ & 73 & 0.74 \\
\hline Rice & 19 & 36 & 17 & $(88)$ & 30 & 0.39 \\
\hline Vegetables & 160 & 83 & 189 & $(118)$ & 69 & 0.38 \\
\hline Fruits & 207 & 107 & 189 & (91) & 114 & 0.60 \\
\hline Citrus íruits & 67 & 59 & 72 & $(107)$ & 70 & 0.68 \\
\hline Other fruits & 140 & 81 & 117 & $(84)$ & 91 & 0.60 \\
\hline Bread & 134 & 54 & 159 & (119) & 70 & 0.80 \\
\hline Milk and milk products & 363 & 220 & 311 & $(86)$ & 192 & 0.60 \\
\hline Cheese & 33 & 20 & 21 & $(64)$ & 15 & 0.61 \\
\hline Eggs & 20 & 13 & 15 & $(75)$ & 10 & 0.61 \\
\hline Meat & 99 & 38 & $9 ?$ & (98) & 36 & 0.46 \\
\hline Meat products & 20 & 16 & 12 & $(57)$ & 11 & 0.54 \\
\hline Fish & 19 & 23 & 11 & $(58)$ & 12 & 0.53 \\
\hline Other sandwich filling $\ddagger$ & 15 & 12 & 11 & $(71)$ & 11 & 0.68 \\
\hline Added fats & 45 & 21 & 47 & (103) & 25 & 0.57 \\
\hline Added sugar & 19 & 22 & 16 & $(82)$ & 22 & 0.84 \\
\hline Cakes, cookies & 51 & 30 & 28 & $(56)$ & 23 & 0.65 \\
\hline Soup & 72 & 70 & 67 & $(94)$ & 87 & 0.54 \\
\hline Non-alcoholic beverages & 1131 & 420 & 1102 & $(97)$ & 383 & 0.63 \\
\hline Alcoholic beverages & 139 & 222 & 99 & $(71)$ & 138 & 0.89 \\
\hline
\end{tabular}

- Food groups with mean intake less than $10 \mathrm{~g}$ per day are not listed in this table: pulses, cereals, mixed dishes, nuts, snacks, candy and soy products.

$+\%$ of record mean.

$\ddagger$ Including peanut butter, jam and other sweet fillings.

Table 3 visualizes the actual level of and the heterogeneity in nutrient intake that could be discriminated by quintiles derived from the questionnaire. Energy adjustment decreased the range for some nutrients, and for some nutrients the results suggest nonlinear relationships. For example, the questionnaire was not able to separate the two highest quintiles of vitamin $\mathrm{C}$ intake, but could nevertheless discriminate a twofold range. The anomaly in the two highest quintiles of vitamin $C$ intake was likely to be attributed to subjects consuming fresh orange juice who checked both the item on (pressed) oranges and that on orange juice.

Table 4 shows the mean number of blank items and the mean error index of the questionnaire according to study group and participation. The validation group had a lower number of blank items and a slightly lower mean error index at baseline than the reproducibility group. Furthermore, among both study groups, the baseline questionnaires of the participants had significantly less blank items and response errors than those of the non-participants; in both study groups combined, the mean number of blank items for participants was 18.6 versus 22.3 for non-participants, while the mean error index was 3.2 and 3.8 respectively. There was no evidence for an interaction effect 
between participation status and study group. Compared to the baseline questionnaire, the mean error index of the repeated questionnaire was not significantly lowered, indicating the absence of a learning effect attributable to recording of intake. The number of blank items, however, was significantly lower for the repeated questionnaire, particularly in the reproducibility group.

Table 3. Mean nutrient intake assessed by 9-day record according to quintile categories of nutrient intake assessed by questionnaire ( 59 men, 48 women): Cohort Study on Diet and Cancer in the Netherlands, 1987/1988.

\begin{tabular}{|c|c|c|c|c|c|c|c|c|c|c|}
\hline \multirow[t]{2}{*}{ Nutrient } & \multicolumn{5}{|c|}{ Unadjusted } & \multicolumn{5}{|c|}{ Adjusted for energy ${ }^{*}$} \\
\hline & Q & $\mathrm{Q}_{2}$ & Q, & Q. & Q, & $\mathrm{Q}_{1}$ & $\mathrm{O}_{2}$ & Q, & Q. & Q, \\
\hline \multicolumn{11}{|c|}{ Energy (kcal) } \\
\hline Men & 1981 & 2318 & 2395 & 2511 & 2840 & & & & & \\
\hline Women & 1636 & 2047 & 1826 & 2168 & 2274 & & & & & \\
\hline \multicolumn{11}{|c|}{ Protein $(\mathrm{g})$} \\
\hline Men & 78 & 74 & 83 & 83 & 99 & 80 & 75 & 82 & 88 & 93 \\
\hline Women & 58 & 71 & 71 & 76 & 76 & 60 & 69 & 72 & 73 & 78 \\
\hline \multicolumn{11}{|c|}{ Total fat (g) } \\
\hline Men & 84 & 97 & 109 & 113 & 138 & 100 & 104 & 108 & 111 & 117 \\
\hline Women & 71 & 87 & 89 & 93 & 111 & 85 & 84 & 91 & 93 & 95 \\
\hline \multicolumn{11}{|c|}{ Polyunsaturated fat (g) } \\
\hline Men & 12 & 15 & 20 & 21 & 26 & 12 & 17 & 19 & 22 & 23 \\
\hline Women & 10 & 14 & 13 & 18 & 20 & 11 & 10 & 15 & 17 & 21 \\
\hline \multicolumn{11}{|c|}{ Cholesterol (mg) } \\
\hline Men & 253 & 284 & 343 & 414 & 455 & 272 & 318 & 343 & 352 & 465 \\
\hline Women & 219 & 287 & 285 & 338 & 396 & 257 & 272 & 308 & 335 & 351 \\
\hline \multicolumn{11}{|c|}{ Mono-, disaccharides (g) } \\
\hline Men & 75 & 103 & 126 & 124 & 155 & 82 & 103 & 122 & 128 & 147 \\
\hline Women & 77 & 103 & 101 & 115 & 138 & 88 & 98 & 102 & 116 & 130 \\
\hline \multicolumn{11}{|c|}{ Polysaccharides (g) } \\
\hline Men & 94 & 111 & 127 & 139 & 169 & 99 & 116 & 127 & 144 & 154 \\
\hline Women & 77 & 100 & 99 & 116 & 112 & 87 & 95 & 100 & 102 & 118 \\
\hline \multicolumn{11}{|c|}{ Dietary fiber $(\mathrm{g})$} \\
\hline Men & 20 & 24 & 26 & 29 & 37 & 20 & 24 & 25 & 29 & 37 \\
\hline Women & 19 & 23. & 26 & 24 & 27 & 20 & 23 & 24 & 24 & 29 \\
\hline \multicolumn{11}{|c|}{ Alcohol (g) } \\
\hline Men & 1 & 6 & 14 & 27 & 36 & 1 & 7 & 14 & 28 & 35 \\
\hline Women & 0 & 3 & 4 & 11 & 24 & 1 & 3 & 4 & 12 & 24 \\
\hline \multicolumn{11}{|c|}{ Calcium (mg) } \\
\hline Men & 874 & 1065 & 1012 & 1084 & 1481 & 934 & 925 & 1192 & 1018 & 1463 \\
\hline Women & 756 & 1026 & 983 & 1146 & 1297 & 799 & 945 & 1015 & 1106 & 1332 \\
\hline \multicolumn{11}{|c|}{ Vitamin A. $(\mathrm{mg}$ eq. $) \dagger$} \\
\hline Men & 0.76 & 0.97 & 0.89 & 1.03 & 1.32 & 0.87 & 0.86 & 0.97 & 1.07 & 1.21 \\
\hline Women & 0.76 & 0.81 & 0.90 & 0.87 & 1.16 & 0.75 & 0.87 & 0.93 & 0.93 & 1.02 \\
\hline \multicolumn{11}{|c|}{ Vitamin C (mg) } \\
\hline Men & 59 & 81 & 87 & 103 & 94 & 53 & 86 & 92 & 100 & 93 \\
\hline Women & 75 & 102 & 101 & 138 & 141 & 75 & 93 & 108 & 139 & 142 \\
\hline \multicolumn{11}{|c|}{ Fat, $\%$ of energy intake } \\
\hline Men & 34 & 40 & 39 & 43 & 44 & 38 & 37 & 39 & 42 & 43 \\
\hline Women & 38 & 39 & 39 & 43 & 45 & 38 & 40 & 40 & 42 & 44 \\
\hline
\end{tabular}

- Adjusted intakes for record and questionnaire nutrients were calculated' as residuals from regression analyses of nutrient on energy intake, to which mean nutrient intake was added.

$\dagger \mathrm{mg}$ equivalents: retinol $(\mathrm{mg})+\mathrm{B}$-carotene $(\mathrm{mg}) / 6$. 
Table 4. Mean number of blank items and mean error index* of baseline questionnaire and (repeated) questionnaire by study group and participation status: Cohort Study on Diet and Cancer in the Netherlands, $1987 / 1988$.

Study group/

participation status
Number of blank items

Baseline

$-$

Mean

SD
Repeated

Mean SD
Error index

Baseline Repeated

Mean SD Mean

Validation study group

\begin{tabular}{|c|c|c|c|c|c|c|c|c|}
\hline All subjects $(n=199) \dagger$ & 18.1 & 25.8 & & & 3.3 & 2.3 & & \\
\hline Participanis $(\mathrm{n}=104)$ & 16.3 & 25.5 & 14.58 & 23.9 & 3.0 & 1.9 & 2.9 & 1.9 \\
\hline Non-participants $(\mathrm{n}=95)$ & $20.0 \ddagger$ & 26.2 & & & $3.5 \ddagger$ & 2.6 & & \\
\hline \multicolumn{9}{|c|}{ Reproducibility study group } \\
\hline All subjects $(\mathrm{n}=373)$ & 20.8 & 27.5 & & & 3.4 & 2.4 & & \\
\hline Participants $(\mathrm{n}=281$ ) & 19.4 & 26.2 & 15.78 & 24.2 & 3.2 & 2.4 & 3.5 & 2.2 \\
\hline Non-participants $(\mathrm{n}=92)$ & $25.1 \neq$ & 30.7 & & & $4.1 \neq$ & 2.4 & & \\
\hline
\end{tabular}

- Error index is the sum of 15 scores each representing an inconsistency or other response error in the completed questionnaire $(0=$ no error, $1=$ moderate error, $2=$ serious error $)$. Since the value of 2 was not assigned for 7 out of 15 variables, the maximum score that could theoretically be attained was 23; actually, the highest score encountered in the cohort was 14 . The error index did not include the number of blank items. Its exact composition is available on request.

+ Data refer to accepted questionnaires.

\# Significant difference $\left(p<0.05, \log _{e}\right.$-transformed data) between participants and non-participants, both study groups combined. There was no evidence for an interaction effect between participation status and study group.

$\$$ Significant difference $(p=0.001$, paired t-test) between baseline and repeated questionnaire, both study groups combined.

Table 5 shows some implications of the validation study results for the cohort as a whole. The Pearson correlation coefficients for intake of the major nutrients, adjusted for energy and sex as presented in Table 1, were adjusted for the distribution of the error index in the questionnaires of the cohort, i.e. the baseline questionnaires of the random reproducibility sample. Based on the dichotomized error index, 21 percent of the questionnaires of the validation study participants appeared to fall within the high error group, compared to 32 percent of the baseline questionnaires of the random reproducibility sample. The anticipated decrease in correlation coefficients was small and appeared to be mainly restricted to cholesterol and vitamin $\mathrm{C}$.

There was no need to adjust for the difference in number of blank items between the two groups, since it resulted in minor (less than 1 percent), non-significant differences in nutrient intake, which was considered as a measure of underreporting.

The attenuation of the correlation coefficients due to the relatively low number of nine recording days is demonstrated by the effect of correction for day-to-day variation in the record data (Table 5). Although the 95 percent confidence intervals became somewhat wider, correlation coefficients increased on average by 0.07 . Due to their relatively large day-to-day variation, the effects of de-attenuation were most pronounced for cholesterol and vitamins $\mathrm{A}$ and $\mathrm{C}$. 
Table 5. Pearson correlation coefficients between nine-day diet record and questionnaire for nutrient intake (adjusted for energy and sex), after correction for error index of the questionnaire and day-to-day variation in the record ( 59 men and 48 women): Cohort Study on Diet and Cancer in the Netherlands, $1987 / 1988$.

\begin{tabular}{|c|c|c|c|c|c|}
\hline \multirow[t]{2}{*}{ Nutrient } & \multicolumn{2}{|c|}{$\begin{array}{l}\text { Correlation } \\
\text { between record } \\
\text { and questionnaire" }\end{array}$} & \multirow{2}{*}{$\begin{array}{l}\text { Adjusted } \\
\text { for error } \\
\text { indext } \\
\text { r }\end{array}$} & \multicolumn{2}{|c|}{$\begin{array}{l}\text { Adjusted for error } \\
\text { inder and day-to-day } \\
\text { variation }\end{array}$} \\
\hline & $\mathrm{r}$ & $(95 \% \mathrm{CI})$ & & $r \neq$ & $(95 \% \mathrm{CI}) \S$ \\
\hline Protein & 0.59 & $(0.45-0.70)$ & 0.58 & 0.64 & $(0.48-0.75)$ \\
\hline Total fat & 0.52 & $(0.37-0.65)$ & 0.49 & 0.53 & (0.36-0.67) \\
\hline Polyunsaturated fat & 0.75 & $(0.65-0.82)$ & 0.76 & 0.80 & $(0.70-0.87)$ \\
\hline Cholesterol & 0.62 & $(0.49-0.72)$ & 0.56 & 0.67 & $(0.48-0.80)$ \\
\hline Mono-, disaccharides. & 0.79 & $(0.71-0.85)$ & 0.80 & 0.83 & $(0.75-0.89)$ \\
\hline Polysaccharides & 0.79 & $(0.71-0.85)$ & 0.80 & 0.84 & $(0.75-0.89)$ \\
\hline Dietary fiber & 0.74 & $(0.64-0.82)$ & 0.73 & 0.79 & $(0.67-0.87)$ \\
\hline Alcohol & 0.86 & $(0.80-0.90)$ & 0.83 & 0.86 & $(0.79-0.91)$ \\
\hline Calcium & 0.62 & $(0.49-0.72)$ & 0.62 & 0.66 & $(0.51-0.76)$ \\
\hline Vitamin A & 0.48 & $(0.32-0.61)$ & 0.52 & 0.76 & $(0.41-0.91)$ \\
\hline Vitamin C & 0.55 & $(0.40-0.67)$ & 0.50 & 0.58 & $(0.39-0.72)$ \\
\hline Mean & 0.66 & & 0.65 & 0.72 & \\
\hline
\end{tabular}

* Derived from Table 1.

$\dagger$ See methods section (data analysis) for adjusiment procedure.

$\ddagger$ According to Beaton et al. (19).

$\S$ According to Rosner and Willell (20).

\section{Discussion}

We have validated a self-administered dietary questionnaire for use in a large-scale prospective cohort study on diet and cancer in the Netherlands. A number of parameters are available to evaluate the validity of an instrument or method relative to another method (e.g. 12). We have chosen two of them: the (Pearson and Spearman) correlation coefficient and the distribution of mean nutrient intakes assessed by the record according to quintile categories of intake assessed by the questionnaire. Although the use of a correlation coefficient for validation purposes in general is criticized by some (21), it has some attractive properties relevant to the etiologic purpose of the cohort study: the correlation coefficient reflects the questionnaire's capacity to rank subjects according to exposure (more important than absolute agreement), taking into account the true variation in exposure in the population studied (12). Thus, it is an adequate measure of the performance of the questionnaire in the cohort population to which it is actually applied. Furthermore, it facilitates comparison with other validation studies of self-administered dietary questionnaires.

Compared to a number of other self-administered questionnaires developed for a similar purpose (Table 6), our questionnaire, comprising approximately 150 food items, is comprehensive. This is mainly due to our requirement to rank subjects with respect to both nutrient and energy intake. In our study, the Pearson correlation coefficients, for unadjusted as well as sex- and energy-adjusted intakes, were generally higher than for questionnaires with fewer items, but similar to the Finnish questionnaire. with 276 
items (4). Inspection of Table 6 may lead to the tentative conclusion that the validity of a questionnaire is proportional to its length, although not all questionnaires match this rule $(5,9)$. Of course, also other properties of the questionnaire, such as lay-out, data editing procedures (22) and characteristics of the population (dietary pattern, range in intake, motivation and ability to complete the questionnaire (22)) influence the validity of the questionnaire.

Table 6. Comparison of validation studies of self-administered questionnaires using the diet record as reference method with respect to the intake of some nutrients important in diet and cancer studies (Pearson correlation coefficients):

\begin{tabular}{|c|c|c|c|c|c|c|c|c|}
\hline First author & Willell & Willett & Pietinen & Pietinen & Block & Ṭønneland & Rimm & This study \\
\hline Reference & 2. & 3 & 5 & 4 & 7 & 8 & 9 & \\
\hline Year & 1985 & 1987 & 1988 & 1988 & 1990 & 1991 & 1992 & \\
\hline Sex of subjects & F & $\mathrm{M} \div \mathrm{F}^{*}$ & M & $\mathrm{M}$ & $\mathrm{F}$ & $\mathrm{M}+\mathrm{F}^{*}$ & M & $\mathrm{M}+\mathrm{F}^{*}$ \\
\hline Number of items & 61 & 116 & 44 & 276 & 94 & 92 & 131 & 150 \\
\hline Energy & + & 0.37 & 0.43 & 0.59 & 0.51 & 0.32 & 0.40 & 0.69 \\
\hline \multicolumn{9}{|l|}{ Fat } \\
\hline Unadjusted & 0.39 & 0.57 & 0.42 & 0.60 & 0.60 & 0.41 & 0.52 & 0.69 \\
\hline Energy-adjustedt & 0.53 & 0.59 & 0.47 & 0.52 & & 0.58 & 0.61 & 0.52 \\
\hline \multicolumn{9}{|l|}{ Polyunsaturated fat } \\
\hline Unadjusted & 0.40 & 0.50 & 0.68 & 0.73 & 0.48 & 0.41 & 0.33 & 0.71 \\
\hline Energy-adjusted & 0.48 & 0.28 & 0.77 & 0.76 & & 0.46 & 0.29 & 0.75 \\
\hline \multicolumn{9}{|l|}{ Fiber } \\
\hline Unadjusted & 0.46 & 0.37 & 0.67 & 0.70 & & 0.34 & 0.49 & 0.74 \\
\hline Energy-adjusted‡ & 0.58 & 0.65 & 0.61 & 0.73 & & 0.46 & 0.64 & 0.74 \\
\hline \multicolumn{9}{|l|}{ Calcium } \\
\hline Unadjusted & & 0.42 & & 0.62 & 0.56 & 0.38 & 0.52 & 0.60 \\
\hline Energy-adjusted & & 0.57 & & 0.66 & & 0.55 & 0.53 & 0.62 \\
\hline \multicolumn{9}{|l|}{ Vitamin A } \\
\hline Unadjusted & 0.26 & 0.62 & 0.38 & 0.51 & 0.47 & 0.27 & 0.35 & 0.53 \\
\hline Energy-adjusted: & 0.36 & 0.70 & 0.36 & 0.49 & & 0.36 & 0.41 & 0.48 \\
\hline \multicolumn{9}{|l|}{ Vitamiñ C } \\
\hline Unadjusted & 0.63 & 0.34 & 0.40 & 0.59 & 0.56 & 0.55 & 0.64 & 0.54 \\
\hline Energy-adjusted & 0.66 & 0.49 & 0.53 & 0.60 & & 0.58 & 0.68 & 0.55 \\
\hline
\end{tabular}

- Studies with both sexes are adjusted for sex (study 8 based on mean for men and women); study 3 also adjusted for age because of the large age range of the study population (20-54 years).

† Emply entries: no data published.

¥ Adjusted for energy and sex, if applicable; study 3 also adjusted for age.

The correlation coefficients for food groups appeared to be somewhat lower than those for nutrients. Differences in coding of foods between the two methods are partly responsible for this: many of the record data were coded as ingredients from recipes or mixed dishes as opposed to the questionnaire data, which were coded as food product. Consequently, the division between food groups was not always clear, resulting in lower correlations. For some food groups, such as vegetables, the relatively low correlation $(0.38)$ was due to a lack of variation in consumption frequency combined with, in our experience, imprecise estimation of portion size. We have evidence, however, that correlations for specific vegetables will be higher due to larger variation in consumption frequency (15).

The general underestimation by our questionnaire of absolute mean nutrient intake. is more pronounced than for other questionnaires $(2-5,7,9)$. Underreporting, caused by 
an incomplete list of foods and items erroneously left blank, counteracts the effect of overreporting caused by long lists of the same sort of items (22). In this study, overreporting due to long enumerations was likely to have occurred for vegetables, citrus fruits and meat. The consumption frequencies for specific meat types, however, were adjusted to the reported weekly frequency of meat consumption, because the adjustment appeared to increase correlation coefficients for the meat types (15). The overreporting of bread may be due to occasional substitution of bread for other foods, such as crackers.

Like in other studies $(2,4,5,9)$, comparison of the baseline questionnaire with the dietary record revealed that it performed almost as well as the repeated questionnaire, which was actually to be validated. It shows that synchronization of the period of reference for the diet record and the questionnaire was not very important, which is indicative of stable dietary habits over time. This result is reassuring when it is considered that a single measurement has to characterize a subject's long-term dietary intake to link it to cancer risk. It may also indicate the absence of a training effect of the diet record keeping which has been suggested by some.

A criticism of validation studies is that the participants are highly motivated and will do better than the population in which the method has been applied at large. This is a particular problem when response to the validation study is relatively low such as for this and other study populations that were not selected for high motivation from the very start $(9,23)$. Indeed, the percentage of questionnaires rejected for incompleteness was 6.0 for the baseline questionnaires of the cohort and 4.7 and 1.8 for the questionnaires repeated in the reproducibility study and the validation study respectively, whereas the number of blank items in accepted questionnaires was also lower for the participants. Similarly, subjects who were willing to participate in the validation study or the reproducibility study had less errors in their baseline questionnaires already. Apparently, subjects who have more problems with the questionnaire or have completed it somewhat carelessly, are less inclined to participate for a second time, in particular in a demanding method like a diet record.

Lack of comparability of the study groups with respect to completeness of the questionnaires is largely solved by exclusion of incomplete questionnaires from all analyses according to identical criteria. Mareover, the difference regarding the number of blank items within accepted questionnaires did not resuit in differential underreporting. Adjustment for the impact of the difference between the two groups in the error index, which is conceptually more directly related to the questionnaire's performance than, for example, nutrient intake and level of education, has shown that selection of the validation study group did not appear to influence the generalizability of the results to the cohort at large.

The adjustment for intraindividual variation in nutrient intake as determined by the record shows that some of the observed correlation coefficients were attenuated by the relatively small number of nine recording days. Vitamins $A$ and $C$ have both relatively low observed correlations. However, the low correlation of vitamin A apparently has been caused by the high day-to-day variation in the record data, while vitamin $C$ assessment depends more on questionnaire performance as was also suggested by the quintile analysis.

In conclusion, we have shown that the questionnaire is able to rank subjects adequately according to intake of the food groups and nutrients investigated. Although the validation study group differed from the cohort with respect to completeness and quality of their questionnaires, this appeared to be no major threat to the generalizability of the validation study's results to the cohort. 


\section{References}

1. Jain $\mathrm{MG}_{\mathrm{w}}$, Harrison $\mathrm{L}$, Howe GR, Miller AB. Evaluation of a self-administered dietary questionnaire for use in a cohort study. Am j Clin Nutr 1982; 36: 931-935.

2. Willett WC, Sampson L, Stampfer MJ, Rosner B, Bain C, Witschi J, Hennekens CH, Speizer FE. Reproducibility and validity of a semiquantitative food frequency questionnaire. Am J Epidemiol 1985: 122: 51-65.

3. Willett WC, Reynolds RD, Cottrell-Hoehner S, Sampson L. Browne ML. Validation of a semiquantitative food frequency questionnaire: comparison with a l-year diet record. J Am Diet Ass 1987; 87: 43-47.

4. Pietinẹn P, Hartman AM, Haapa E, Rãsănen L, Haapakoski J, Palmgren J, Albanes D, Virtamo J, Hutrunen IK. Reproducibility and validity of dietary assessment instruments: a self-administered food use questionnaire with a portion size picture booklet. Am J Epidemiol 1988; 128: 655-666.

5. Pietinen $\overline{\mathrm{P}}$, Harıman $\mathrm{AM}$, Haapa $\mathrm{E}$, Räsinen $\mathbf{L}$, Haapakoski J, Palmgren J, Albanes D, Virtamo $\mathrm{J}_{\mathrm{a}}$ Huttunen JK. Reproducibility and validity of dietary assessment instruments: a qualitative food frequency questionnaire. Am J Epidemiol 1988; 128: 667-676.

6. Salvini S, IIunter DJ, Sampson L, Stampfer MJ, Colditz GA, Rosner B, Willett WC. Food-based validation of a dietary questionnaire: the effects of week-to-week variation in food consumption. Int J Épidemiol 1989; 18: 858-867.

7. Block G, Woods M, Potosky A, Clifford C. Validation of a self-administered diel history questionnaire using multiple diet records. J Clin Epidemioll 1990; 43: 1327-1335.

8. Tjanneland A, Overvad KIM, Haraldsdótir J, Bang S, Eweriz M, Jensen OM. Validation of a semiquantitative food frequency questionnaire developed in Denmark. Int J Epidemiol 1991; 20: 906-912

9. Rimm EB, Giovannucci EL, Stampfer MJ, Colditz GA, Litin LB, Willet WC. Reproducibility and validity of a expanded self-administered semiquantitative food frequency questionnaire among male health professionals. Am J Epidemiol 1992; 135: 1114-1126.

10. Bausch-Goldbohm RA, van den Brandt PA, van ' $t$ Veer $P$, Sturmans F, Hermus RJJ. Results of the methodological study for the design of a simplified, self-administered questionnaire. In: Riboli $\mathrm{E}_{\text {, }}$ Saracci R, eds. Diet, hormones and cancer: methodological issues for prospective studies. Lyon, France: International Agency for Research on Cancer 1988; 79-89, IARC Technical Report No. 4.

11. Van den Brandt PA, Goldbohm RA, van 'I Veer P, Volovics A, Hermus RJJ, Sturmans F. A largescale prospective cohort study on diet and cancer in the Netherlands. J Clin Epidemiol 1990; 43: 285-295.

12. Willett WC. Reproducibility and validity of food-frequency questionnaires. In: Willett WC, ed. Nutritional Epidemiology. New York/Oxford: Oxford University Press 1990; 92-126.

13. Results of a panel survey: fresh vegetables and fruits [in Dutch]. The Hague, the Netherlands: Produktschap voor Groenten en Fruit, Market Research Department, 1986.

14. Results of a panel survey by NIAM on the consumption of meat and meat products [in Dutch] Rijswijk, Netherlands: Voorlichtingsbureau Vlees, 1986.

15. Den Breeijen H. Validation of the draft version of a self-administered questionnaire used in a cohort study on diet and cancer [in Dutch]. TNO report V 90.019. Zeist, Netherlands: TNO Toxicology and Nutrition Institute, 1990.

16. Livingstone MBE. Prentice AM, Strain JJ, Coward WA, Black AE, Barker ME, McKenna PG, Whitehead RG. Accuracy of weighed dietary records in studies of diet and health. Br Med J 1990; 300: $708-712$

17. NEVO tabel. Duich food composition table 1986-1987. The Haguc, the Netherlands: Voorlichtingsbureau voor de Voeding, 1986.

18. Nelson $\mathrm{M}$, Black $\mathrm{AE}$, Morris JA, Cole TJ. Between- and within-subject variation in nutrient intake. from infancy to old age: estimating the number of days required to rank dietary intakes with desired precision. Am J Clin Nutr 1989; 50: 155-167.

19. Beaton GH, Milner J, Corey P, McGuire V, Cousins M. Stewart E, Ramos M de, Hewitt D, Grambsch PV, Kassin N, Little JA. Sources of variance in 24-hour dietary recall data: implications for nutrition study design and interpretation. Am J Clin Nutr 1979; 32: 2546-2559.

20. Rosner B. Willelt WC Intervall estimates for correlation coefficients corrected for within-person variation: implications for study design and hypothesis testing. Am. J Epidemio! 1988; 127: 377-386.

21. Bland JM, Altiman. DG. Statistical methods for assessing agreement between two methods of clinical measurement. Lancet 1986; 307-310.

22. Block G. Hartman AM. Issues in reproducibility and validity of dietary studies. Am J Clin Nutr 1989; 50: 1133-1138.

23. Hankin JH, Wilkens LR, Kolonel LN, Yoshizawa CN. Validation of a quantitative diet history method in Hawaii. Am J Epidemiol 1991; 133: 616-628. 


\title{
Chapter 6
}

\section{Reproducibility of a food frequency questionnaire and stability of dietary habits determined from five annually repeated measurements*}

\section{R. Alexandra Goldbohm ${ }^{1,2}$, Pieter van ' $t$ Veer $^{1}$, Piet A. van den Brandt ${ }^{2}$,} Martin A. van 't Hof ${ }^{3}$, Henny A.M. Brants', Ferd Sturmans ${ }^{2}$, Rudolph J.J. Hermus'.

1) TNO Toxicology and Nutrition Institute, Zeist, the Netherlands; 2) University of Limburg Department of Epidemiology, Maastricht, the Netherlands; 3) University of Nijmegen, Department of Medical Statistics, Nijmegen, the Netherlands

\begin{abstract}
The reproduclbility of a $\mathbf{1 5 0 - i t e m ~ f o o d ~ f r e q u e n c y ~ q u e s t i o n n a l r e ~ ( F F Q ) , ~ w h i c h ~ h a s ~ b e e n ~}$ used to assess dietary habits in a cohort study on diet and cancer among 120,000 subjects, was determined from five annually repeated questionnaire administrations in independent random samples from the cohort. Pearson correlation coeficlents between the baseline and the repeated measurement of nutrient intake were calculated for each time interval, l.e. ranging from one to five years. Linear regression of the correlation coefricients on time interval provided estimates of the test-retest correlation of the FFQ (intercept of the regression line) and of the decline in correlation over time (slope).

The test-retest correlation averaged over all nutrients was 0.66 . The average decllne In correlation amounted to $\mathbf{0 . 0 7}$ after nve years, indicating that the potentlal of the FFQ measurement to rank subjects according to nutrient intake Is maintalned relatively well over time. It is concluded that a single baseline measurement of the FFQ is a good indicator of nutrient intake over a period of at least five years.
\end{abstract}

- Submitted for publication 


\section{Introduction}

Food frequency questionnaires are often the method of choice for assessing dietary habits in large-scale epidemiological studies, such as a prospective cohort study. It is generally recognized, however, that it has to be validated against a standard reference method of dietary assessment, such as a diet record $(1,2)$. It is furthermore considered desirable to assess the reproducibility of the instrument by repeating the questionnaire administration, although such information is much less needed in the presence of data from a validation study. In the latter situation repeated measurements are mainly useful to assess changes in dietary habits over time $(1,2)$.

Most studies on reproducibility of food frequency questionnaires or dietary histories have repeated the measurement once (3-7) or, occasionally, twice (8). From these studies, it is difficult to deduce whether the imperfect reproducibility is caused by the measurement ептог of the instrument, changes in dietary habits between the administrations, or both. A further complicating problem is that part of the measurement error may not be random, but correlated between two measurements by the same instrument resulting in spuriously high reproducibility. This happens when an error associated with the questionnaire recurs systematically for a subject (9).

We evaluated the reproducibility of a self-administered food frequency questionnaire (FFQ) that has been used for baseline assessment of exposure in a large prospective cohort study on diet and cancer (10). The questionnaire has been validated against a 9-day diet record (Goldbohm et al., submitted for publication). While the validation study provided insight into the measurement error of the questionnaire, we also wished to know how well one measurement was able to characterize the long-term dietary habits of the participants in the cohort study. Therefore, we repeated the questionnaire administration annually during the first five years of follow-up in random independent samples of the cohort. The relatively large number of repetitions allows us to separate the effect of changes in dietary habits over time from the (pure) test-retest error of the questionnaire. This type of information is relevant for the interpretation (or correction for attenuation) of the associations between dietary habits as assessed by the FFO and the outcome, i.e. the risk of cancer.

A second purpose of this study was to combine the results from the validation and the reproducibility study to elucidate to what extent the measurement error is correlated between two repeated measurements. Although this information is less relevant to the interpretation of the diet-cancer relation, it may provide more insight into the composition of the measurement error associated with a food frequency; questionnaire, and thus open ways to improve methods in future studies.

\section{Subjects and methods}

\section{Subjects and design}

The prospective cohort study on diet and cancer has been initiated in the Netherlands in Septembei 1986. The cohort included 58,279 men and 62,573 women aged 55-69 at the start of the study. At baseline, the cohort members completed a mailed, self-administered questionnaire, which included the food frequency questionnaire. A subcohort of 3500 subjects, randomly sampled from the cohort after baseline measurement, was followed up for vital status biennially by means of short mailed questionnaires. The subjects for the reproducibility study were randomly sampled from the subcohort and comprised five independent samples of 400 subjects (200 men and 200 women), one for each year in which the food frequency 
questionnaire was repeated. The repeated questionnaires were mailed in the same month (September) as the baseline questionnaire in each following year (1987 to 1991).

\section{Food frequency questionnaire}

The dietary questionnaire and its processing have been described in detail by Goldbohm et al. (submitted for publication). The questionnaire's most important characteristics are summarized here. It assessed habitual consumption of 150 food items during the past year. Respondents could choose one of seven frequency categories ranging from never/less than once per month to 6-7 times per week. The number of servings per consumption frequency was asked in natural or household units (e.g., glass). Questions on vegetables were specified with respect to season (summer and winter). Margarine used on bread and cooking fats and oils were specified as to type and brand in open questions. An open-ended question also asked to list any foods eaten regularly (at least once a week) but not included in the questionnaire. The repeated questionnaires included an additional question on the changes in dietary habits perceived by the respondent since the baseline administration.

Questionnaires were double-keyed and checked for completeness, consistency, range and other response errors. In the subcohort, 7.0 percent of the baseline questionnaires were considered unacceptable according to formal criteria for incompleteness or inconsistency (Goldbohm et al., submitted for publication). Nutrient intake was calculated using the computerized Dutch food composition table (11).

\section{Data analysis}

Participants of the reproducibility study were excluded from data analysis when their baseline or repeated questionnaire was considered unacceptable for reasons described above. Nutrient intakes were log-transformed to improve their distribution towards normality. An alcohol intake of 0 gram per day was replaced with 0.1 gram before transformation. Energy adjustment of nutrient intakes was done by means of regression analysis according to Willett and Stampfer (12).

The analyses referred to the intake of a number of nutrients considered relevant for diet and cancer studies (see Results). Pearson correlation coefficients were calculated between the baseline and each repeated measurement. The five resulting data points (correlation coefficients), i.e. one for each time interval, were regressed on time interval since baseline. The slope of the resulting regression line is an indicator of the intraindividual difference in change in nutrient intake over time, while the intercept $\left(\mathbf{r}_{0}\right)$ provides an estimate of the reproducibility of the measurement as if no time had elapsed between these measurements (13). For comparison of the measurement error between subgroups of the study population, we calculated the standard deviations of the differences between baseline and repeated measurements.

Finally, the variance of nutrient intakes as assessed by the FFQ was divided into between-subject and within-subject variance. The latter is to be considered as error variance of the FFQ. The ratio of within- to between-subject variance of the FFQ was estimated from the Pearson correlation coefficient between the FFQ and reference method used in the validation study (9-day diet record), adjusted for the within-subject (day-to-day) variation in the diet record (14). Subsequently, the error (within-subject) variance of the FFQ was divided into random and correlated error variance (14). The error correlated between two FFQ measurements was calculated from the data of the validation and the reproducibility study combined. First, the expected (in the absence of correlated error) test-retest correlation was calculated from the validation study as the square of the correlation coefficient between FFQ and record, corrected for day-to-day variation in the record. Then, the expected correlation was subtracted from the 
correlation observed in the reproducibility study; the resulting difference represents the proportion of correlated error variance of the total variance observed by the FFQ.

\section{Results}

Table 1 shows the response to the repeated questionnaire administrations after correcting the samples for uneligible subjects (i.e. deceased and moved to an unknown address). The overall response during the five years was $82 \%$. Neither a trend with increasing time interval nor relevant differences between men and women were detected. After exclusion of subjects with unacceptable (i.e. incomplete or inconsistent) baseline and/or repeated questionnaires, $74.9 \%$ of the eligible subjects remained for data analysis. Among the responders, the mean percentage of unacceptable baseline questionnaires was $5.3 \%$. The proportion of responders with acceptable baseline, but unacceptable repeated questionnaires increased from $1.5 \%$ in 1987 to $5.6 \%$ in 1991.

Table 1. Response to the annually repeated questionnaire administrations.

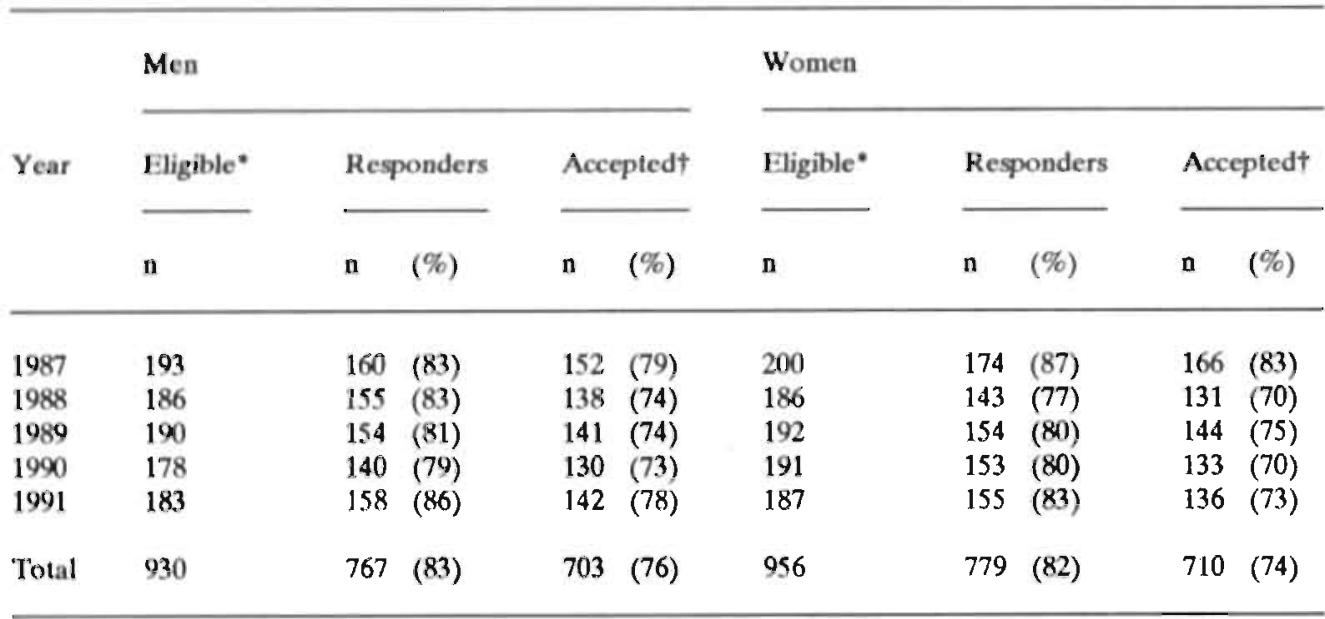

- Sample sizes at baseline: 200; deceased subjects and subjects with unknown address were considered ineligible.

† Inçomplete and inconsistent questionnaires cxcluded.

Table 2 presents descriptive data on nutrient intake in the study population as assessed by the baseline FFQ. Evaluation of the mean nutrient intakes over time revealed very weak trends, which were compatible with differences between age groups, i.e. among both sexes there was a tendency to decrease energy and energy-adjusted fat intake (with the exception of polyunsaturated fat), which was compensated with protein (men) or carbohydrates (women).

The separation of the intraindividual change over time from the reproducibility of the FFQ is illustrated in Figure 1 for calcium intake in women. The mean correlation coefficient over five time intervals is 0.63 . Linear regression of the interval-specific correlation coefficients on time interval resulted in a regression line that had a slightly negative slope and its intercept $\left(r_{0}\right)$ at 0.69 . The slope indicates a decline in the correlation coefficient of 0.02 per year. 
Table 2. Mean and standard deviation of nutrient intake as assessed by the baseline FFQ and trend (\% of baseline mean in five years) ${ }^{*}$ for men and women.

\begin{tabular}{|c|c|c|c|c|c|c|}
\hline \multirow[b]{2}{*}{ Nutrient } & \multicolumn{3}{|c|}{ Men $(n=703)$} & \multicolumn{3}{|c|}{ Women $(\mathrm{n}=710)$} \\
\hline & Mean & SD & Trend $(\%)$ & Mean & SD & Trend (\%) \\
\hline Energy (kcal) & 2134 & 470 & -4 & 1670 & 395 & -1 \\
\hline Protein (g) & 75.0 & 16.5 & 3 & 65.3 & 15.3 & -2 \\
\hline Animal & 47.6 & 133 & 5 & 43.7 & 12.8 & -2 \\
\hline Vegetable & 28.0 & 83 & 0 & 22.0 & 6.5 & -2 \\
\hline Fat (g) & 92.7 & 27.4 & -1 & 73.6 & 23.6 & -3 \\
\hline Saturated & 36.4 & 11.4 & -3 & 29.5 & 9.9 & -5 \\
\hline Monounsaturated & 34.9 & 11.1 & -3 & 27.6 & 9.6 & -5 \\
\hline Polyunsaturated & 19.5 & 9.5 & 6 & 14.9 & 7.5 & 8 \\
\hline Cholesterol (mg) & 275 & 88 & -5 & 234 & 71 & -10 \\
\hline Carbohydrates $(\mathrm{g})$ & 222.7 & 62.4 & 0 & 176.5 & 47.2 & 2 \\
\hline Mono/disaccharides & 101.2 & 41.4 & -1 & 84.0 & 32.0 & 3 \\
\hline Polysaccharides & 120.7 & 36.1 & 0 & 92.3 & 25.6 & 2 \\
\hline Alcohol (g) & 14.7 & 15.4 & 1 & 5.4 & 8.5 & 23 \\
\hline Dietary fiber $(\mathrm{g})$ & 28.8 & 8.7 & -2 & 25.1 & 7.1 & -5 \\
\hline Vitamin A (mg eq)t & 1.02: & 0.41 & -7 & 0.89 & 0.38 & -19 \\
\hline B-carotene (mg eq) & 0.41 & 0.22 & 0 & 0.42 & 0.23 & -12 \\
\hline Retinol (mg) & 0.61 & 0.34 & -12 & 0.47 & 0.27 & -26 \\
\hline Vitamin $C(\mathrm{mg})$ & 98.9 & 41.4 & -6 & 107.6 & 45.1 & -1 \\
\hline Calcium (mg) & 937 & 317 & 7 & 895 & 312 & 0 \\
\hline Selenium ( $\boldsymbol{\mu g}$ ) & 61.1 & 15.9 & 1 & 53.6 & 14.9 & -5 \\
\hline
\end{tabular}

- Trend: regression coefficient of the (energy-adjusted) difference between baseline and repeated measurement regressed on time interval (5 years), expressed as \% of the baseline mean. A negative value denotes a decreasing trend.

$\dagger$ retinol equivalents: $B$-carotene $(\mathrm{mg}) / 6+$ retinol $(\mathrm{mg})$.

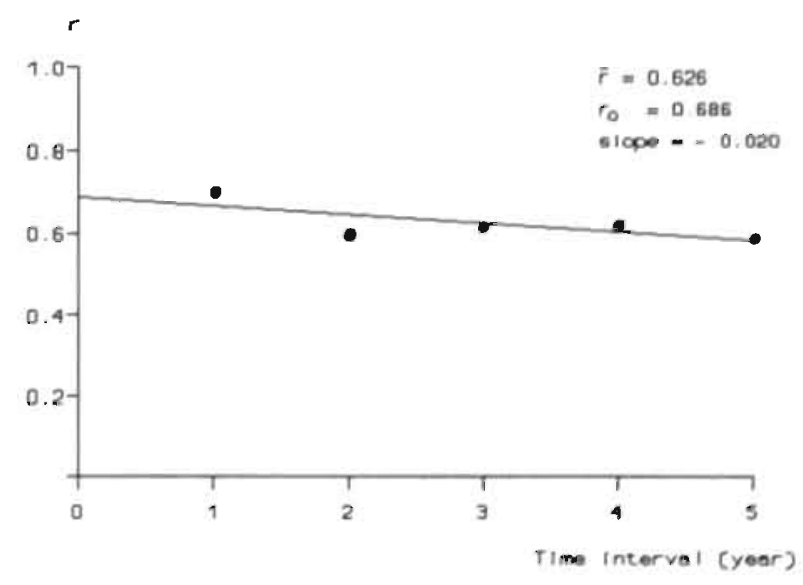

Figure 1. Example of the regression of the Pearson correlation coefficients between two measurements of the FFQ on time interval between the measurements. This example pertains to calcium intake in women. 
Table 3. Mean Pearson correlation coefficients $\left(\mathrm{r}_{\mathrm{m}}\right)$ between paired measurements of nutrient intake by FFQ and parameters of their regression on time interval.

\begin{tabular}{|c|c|c|c|c|c|c|}
\hline \multirow[b]{2}{*}{ Nutrient } & \multicolumn{3}{|c|}{ Unadjusted } & \multicolumn{3}{|c|}{ Energy-adjusted } \\
\hline & $\mathrm{r}_{\mathrm{m}}$ & $r_{0}^{*}$ & Res.SD† & $r_{m}$ & $\mathrm{r}_{0}{ }^{*}$ & Res.SD ${ }^{\dagger}$ \\
\hline & \multicolumn{6}{|c|}{ Men } \\
\hline Energy & 0.68 & 0.63 & 0.056 & - & - & - \\
\hline Protein & 0.57 & 0.57 & 0.041 & 0.52 & 0.61 & 0.036 \\
\hline Animal & 0.55 & 0.54 & 0.108 & 0.54 & 0.61 & 0.092 \\
\hline Vegetable & 0.66 & 0.70 & 0.050 & 0.63 & 0.65 & 0.076 \\
\hline Fat & 0.63 & 0.58 & 0.097 & 0.58 & 0.56 & 0.104 \\
\hline Saturated & 0.64 & 0.62 & 0.074 & 0.62 & 0.69 & 0.099 \\
\hline Monounsaturated & 0.64 & 0.64 & 0.081 & 0.60 & 0.65 & 0.096 \\
\hline Polyunsaturated & 0.64 & 0.60 & 0.099 & 0.64 & 0.63 & 0.065 \\
\hline Cholesterol & 0.66 & 0.61 & 0.071 & 0.66 & 0.62 & 0.073 \\
\hline Carbohydrates & 0.71 & 0.72 & 0.044 & 0.70 & 0.72 & 0.044 \\
\hline Mono/disaccharides & 0.68 & 0.70 & 0.057 & 0.63 & 0.72 & 0.054 \\
\hline Polysaccharides & 0.68 & 0.74 & 0.079 & 0.67 & 0.72 & 0.044 \\
\hline Alcohol & 0.85 & 0.90 & 0.044 & 0.85 & 0.89 & 0.046 \\
\hline Dietary fiber & 0.68 & 0.71 & 0.047 & 0.69 & 0.78 & 0.034 \\
\hline Vitamin $A$ & 0.51 & 0.48 & 0.065 & 0.49 & 0.54 & 0.077 \\
\hline B-carotene & 0.55 & 0.52 & 0.037 & 0.54 & 0.52 & 0.029 \\
\hline Retinol & 0.46 & 0.50 & 0.061 & 0.43 & 0.57 & 0.085 \\
\hline Vitamin $C$ & 0.62 & 0.67 & 0.042 & 0.63 & 0.69 & 0.042 \\
\hline Calcium & 0.59 & 0.67 & 0.075 & 0.59 & 0.73 & 0.088 \\
\hline Selenium & 0.58 & 0.63 & 0.058 & 0.57 & 0.64 & 0.143 \\
\hline \multirow[t]{2}{*}{ Mean $\downarrow$} & 0.635 & 0.649 & & 0.619 & 0.674 & \\
\hline & \multicolumn{6}{|c|}{ Women } \\
\hline Energy & 0.64 & 0.66 & 0.081 & - & - & - \\
\hline Protein & 0.61 & 0.63 & 0.064 & 0.61 & 0.64 & 0.059 \\
\hline Aninal & 0.60 & 0.65 & 0.054 & 0.59 & 0.64 & 0.049 \\
\hline Vegetable & 0.61 & 0.64 & 0.088 & 0.59 & 0.68 & 0.070 \\
\hline Fat & 0.56 & 0.60 & 0.115 & 0.48 & 0.43 & 0.071 \\
\hline Saturated & 0.59 & 0.68 & 0.056 & 0.56 & 0.62 & 0.040 \\
\hline Monounsaturated & 0.57 & 0.60 & 0.104 & 0.58 & 0.61 & 0.048 \\
\hline Polyunsaturated & 0.55 & 0.62 & 0.095 & 0.54 & 0.65 & 0.066 \\
\hline Cholesterol & 0.65 & 0.73 & 0.041 & 0.61 & 0.72 & 0.066 \\
\hline Carbohydrates & 0.67 & 0.67 & 0.040 & 0.61 & 0.57 & 0.041 \\
\hline Mono/disaccharides & 0.65 & 0.68 & 0.065 & 0.59 & 0.66 & 0.050 \\
\hline Polysaccharides & 0.65 & 0.68 & 0.050 & 0.61 & 0.62 & 0.033 \\
\hline Alcohol & 0.84 & 0.87 & 0.010 & 0.84 & 0.87 & 0.019 \\
\hline Dietary liber & 0.66 & 0.69 & 0.064 & 0.67 & 0.76 & 0.067 \\
\hline Vitamin $A$ & 0.52 & 0.66 & 0.061 & 0.49 & 0.67 & 0.033 \\
\hline B-carotene & 0.58 & 0.69 & 0.084 & 0.57 & 0.71 & 0.081 \\
\hline Relinol & 0.47 & 0.56 & 0.102 & 0.40 & 0.43 & 0.085 \\
\hline Vitamin C & 0.64 & 0.71 & 0.062 & 0.64 & 0.73 & 0.056 \\
\hline Calcium & 0.63 & 0.69 & 0.034 & 0.63 & 0.63 & 0.075 \\
\hline Selenium & 0.55 & 0.46 & 0.057 & 0.54 & 0.42 & 0.077 \\
\hline Mean ‡̦ & 0.617 & 0.663 & & 0.597 & 0.650 & \\
\hline
\end{tabular}

- Intercepi of the linear regression line: estimate of r (test-retest) from which the effect of change over time has been removed.

$\uparrow$ Residual standard deviation for regression of $\mathrm{r}$ on time interval.

¥ The following nutrients were included in the calculation of the averages: energy, animal and vegetable protein, saturated, monounsaturated and polyunsaturated fat, cholesterol, mono/disaccharides, polysaccharides, alcohol, dietary fiber, B-carotene, retinol, vitamin C, calcium and selenium. 
Table 3 summarizes the mean correlation coefficients and the regression results for all nutrient intakes. For most nutrients a negative slope was observed, but none of them significantly differed from zero. The decrease in correlation averaged across nutrients ranged from 0.005 to 0.018 per year. The intercept was on average ca. 0.05 higher than the mean correlation, with the exception of the unadjusted intake in men, which showed a smaller difference. The residual standard deviation of the regression ranged from 0.01 (alcohol) to 0.14 (selenium). For most nutrients, it agreed well with the standard error of the mean of the correlation coefficients $(0.07)$, indicating that the fit of the regression lines is according to expectation.

Overall, $13 \%$ of the men and $17 \%$ of the women reported to have changed their dietary habits between the two measurements. These proportions did not increase with increasing time interval. The measurement error of nutrient intakes (expressed as a percentage of mean intake) is displayed in Table 4 for all subjects as well as for those who reported a change in their dietary habits after baseline measurement. The measurement error, which in this table also includes the intraindividual change in dietary habits between two measurements, was consistently higher for the subjects who reported to have changed habits. Adjustment for energy intake consistently decreased the measurement error. The measurement errors were very similar for men and women (data not shown), with the exception of intake of energy and fats, which was ca. $15 \%$ higher for women than for men. Adjustment for energy intake strongly reduced these differences.

Table 4. Pooled measurement error $(\%)$ of nutrient intakes assessed by $F \mathrm{FQ}$ for all subjects and subjects who reported to have changed their dietary habits after baseline.

\begin{tabular}{|c|c|c|c|c|}
\hline \multirow[b]{2}{*}{ Nutrient } & \multicolumn{2}{|c|}{ Unadjusted } & \multicolumn{2}{|c|}{ Energy-adjusted } \\
\hline & All & Changed & All & Changed \\
\hline Energy & 133 & 14.2 & - & . \\
\hline Protein & 14.8 & 15.9 & 10.4 & 11.6 \\
\hline Animal & 20.0 & 23.0 & 16.2 & 17.9 \\
\hline Vegetable & 16.7 & 17.3 & 12.4 & 13.1 \\
\hline Fat. & 19.9 & 22.1 & 10.9 & 14.7 \\
\hline Saturated & 20.7 & 23.8 & 13.0 & 17.3 \\
\hline Monounsaturated & 21.0 & 24.5 & 12.6 & 16.8 \\
\hline Polyunsaturated & 32.0 & 35.4 & 27.1 & 31.6 \\
\hline Cholesterol & 19.9 & 24.7 & 16.7 & 20.9 \\
\hline Carbohydrates & 14.9 & 17.4 & 9.3 & 11.1 \\
\hline Mono/disaccharides & 23.1 & 29.1 & 19.3 & 24.2 \\
\hline Polysaccharides & 163 & 18.7 & 12.2 & 14.7 \\
\hline Alcohol & 77.4 & 84.9 & 75.9 & 83.5 \\
\hline Diẹtary fiber & 16.6 & 17.0 & 13.7 & 143 \\
\hline Vitamin A & 26.0 & 27.2 & 22.9 & 24.6 \\
\hline B-carotene & 32.9 & 31.7 & 32.4 & 31.4 \\
\hline Retinol & 373 & 40.2 & 32.5 & 36.2 \\
\hline Vitamin C & 25.6 & 27.7 & 24.5 & 26.5 \\
\hline Calcium & 21.7 & 25.7 & 18.9 & 22.4 \\
\hline Selenium & 18.0 & 23.2 & 153 & 20.6 \\
\hline
\end{tabular}

Table 5 shows for a limited number of nutrients the results of the partitioning of variance as measured by the FFQ. The ratio of within- to between-subject variance of 
the nutrient intakes was less than 1 , with the exception of the higher ratio for intake of protein, calcium and vitamin C. Adjustment for energy intake did not substantially change the relative size of the variance components, except for fat intake for which the relative contribution to between-subject variance decreased. The nutrients that showed the highest correlated error were, again, protein, calcium, vitamin $\mathrm{C}$ and energyadjusted fat.

Table 5. Components of variance (\%) of nutrient intake as assessed by FFQ (unadjusted and energyadjusted).

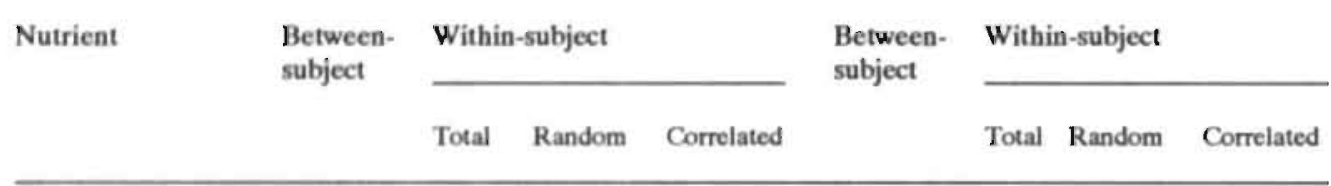

\section{Unadjusted}

Enengy-adjusted

\begin{tabular}{llllrlllr} 
Energy & 53 & 47 & 33 & 14 & & & & \\
Protein & 38 & 62 & 40 & 22 & 37 & 63 & 38 & 25 \\
Fat & 54 & 46 & 39 & 7 & 32 & 68 & 44 & 24 \\
Polyunsaturated fat & 56 & 44 & 39 & 5 & 62 & 38 & 35 & 3 \\
Cholesterol & 58 & 42 & 31 & 11 & 55 & 45 & 33 & 12 \\
Mono/disaccharides & 64 & 36 & 31 & 5 & 68 & 32 & 31 & 1 \\
Polysaccharides & 72 & 28 & 29 & -1 & 68 & 32 & 33 & -1 \\
Dietary fiber & 64 & 36 & 30 & 6 & 65 & 35 & 23 & 12 \\
Alcohol & 78 & 22 & 12 & 10 & 80 & 20 & 12 & 8 \\
Calcium & 40 & 60 & 32 & 28 & 43 & 57 & 32 & 25 \\
Vitamin A & 40 & 60 & 42 & 18 & 49 & 51 & 40 & 11 \\
Vitamin C & 39 & 61 & 31 & 30 & 40 & 60 & 29 & 31 \\
& & & & & & & & \\
\hline
\end{tabular}

\section{Discussion}

We have evaluated the stability of dietary habits over time among participants of the Dutch cohort study on dict and cancer. Mean intakes changed very little. The correlations between two measurements decreased slightly over time, indicating a minor change in the capacity of the baseline (FFQ) measurement to rank subjects within the distribution of nutrient intake.

A matter of potential concern in the interpretation of the results is the representativeness of the participants of the study, in particular those with acceptable questionnaires, relative to the entire cohort. Although response remained high during the study period, the proportion of unacceptable questionnaires increased somewhat with time. This is compatible with the cross-sectionnally observed association between age and proportion of unacceptable questionnaires. As people grow older, they experience apparently more trouble in completing the questionnaire, most likely due to sickness, poor sight or shorter memory. We may thus have included in the reproducibility study subjects who perform better on average than the cohort at large. However, since the percentage of questionnaires that had to be excluded in addition to those already excluded from the baseline measurement in the complete sample is small $(3.2 \%)$, the effect on generalizibility of the study results to the cohort at large is not likely to be serious.

Our data also provided evidence that subjects who consciousily changed their dietary habits have consistently larger measurement errors than those who did not report a 
change. These results are consistent with the assumption that the decreased correlation over time is to be attributed to intraindividual change in dietary habits and have also been observed by others $(15,16)$. It is not immediately clear why the proportion of subjects who reported a change did not increase with time. The most plausible explanations are that they may have forgotten any changes in the more distant past (i.e. more than a year ago), as is consistent with the literature (17-22), or that reported changes may have been temporary. The latter explanation does not fit the data.

To describe the reproducibility of the FFQ and the intraindividual stability of nutrient intake over time, we used the Pearson product-moment correlation coefficient. This statistic depends on both the within-subject measurement error of the instrument and the variation of the measured variable in the population. It therefore adequately describes the ability of the FFO to discriminate between exposure levels within the study population (2). Furthermore, the straightforward statistical properties of the Pearson correlation coefficient facilitate calculation of statistics such as the (pure) testretest error and the intraindividual change over time, as was done in the present analysis. Finally, the Pearson $r$ (or the related regression coefficient) may be used to correct for attenuation present in associations between nutrient intake as assessed by FFQ and outcome $(9,23,24)$. For other commonly used statistics, such as percentage agreement, (weighted) kappa (20) and the intraclass correlation coefficient $(3,4,6-8)$, it has been shown empirically that they are related to the Pearson $r$ (25). Indeed, in reproducibility studies of diet assessment the intraclass and the Pearson correlation coefficient appeared to be very similar $(3,4,6)$. In our study, the intraclass correlation coefficient would be less useful as it also depends on temporal trends in mean intake.

Repeated measurements are relevant since they give information on how well the classification of subjects is maintained over time. This is of particular importance in diet and cancer studies, in which the relevant dietary exposure is presumed to extend over many more years than just the one addressed by the FFQ. Potential alternative approaches to this problem include recall over a longer reference period than one year or a more distant past, but, unfortunately, such approaches tend to be influenced considerably by current habits (17-22). Another, costly approach, which has been used in some prospective studies (26), is to repeat the FFQ administration regularly in the entire cohort. Our results substantiate the initial idea that a single FFO administration is not only relevant to the dietary habits in the previous year, but may extend to a much longer period. The average decline in correlation after five years amounted to 0.07 . Assuming that the same trends in correlation apply to the five years preceding the baseline measurement, we may have been able to quantify the measurement error of the single FFQ measurement with respect to the dietary habits over a ten-year period. Extrapolation of the decrease in correlation beyond the five year period may be considered speculative, but is in line with the literature, which has reported correlation coefficients of 0.30 to 0.40 for dietary assessments 11 to 25 years apart $(17,22)$.

It is not very useful to compare the reproducibility of our FFQ to those reported for other FFQs, since reproducibility is determined in different ways and FFQs may have a varying degree of correlated error. One study, however, assessed the reproducibility of an extensive cross-check dietary history method in the same way as we did, also using 5 repeated measurements $(13,27)$. In that study higher test-retest correlations were found. For example, for calcium intake a $\mathbf{r}_{0}$ of 0.83 was found, indicating a smaller measurement error than observed for our FFQ (under the assumption that the proportion of correlated error is similar for both methods). The slope of the regression line, however, was smaller for the FFQ.

In addition to the assessment of reproducibility of the FFQ and the stability of dietary habits, the data from this study, combined with those from the validation study, 
provided a good opportunity to learn more about the measurement error of the FFQ. Although there appears to be interest in this subject, published data are scarce (14). The method proposed by Beaton (14) to estimate components of variance is attractive, but it relies on a number of assumptions. Since we cannot be sure that the assumptions have been met, the results have to be considered as rough indicators rather than as exact data. For example, one of the assumptions, i.e. the reference (record) method measures true usual intake, may not hold for all nutrients as is made plausible by Hunter et al.(28). Another assumption, required for the calculation of correlated error, is that there is no change in dietary habits between two FFQ measurements. We have met this assumption by using the intercept $\left(\mathrm{r}_{0}\right)$ from the regression of correlations on time interval, instead of the mean $r$. The results indicate that nutrient intakes as assessed by FFQ have a ratio of within- to between-subject variance of less than 1, with the exception of protein, calcium and vitamin C. These are also the nutrients with the largest proportion of correlated error $(>20 \%)$. These results appear to be more favorable for our FFQ than those presented by Wu et al.(29), who have found larger within- to between-subject variance ratios. For our FFQ, energy adjustment did not seriously affect the performance of the questionnaire, nor the composition of the variance. Fat intake appeared to be the only unfavorable exception to this finding. Although the measurement error appeared to decrease substantially after energy adjustment, it did not outweigh the effect of decrease in true between-subject variance.

Repeated measurements in subgroups of the study population are advocated by many authors $(23,24,30,31)$ to correct relative risk estimates for attenuation. The assumption that errors are independent is often made explicitly $(24,32)$. Our data suggest that errors may be correlated between two measurements, although their relative size is small. Beaton (14) has also given some examples of nutrient intakes assessed by FFQ that have larger correlated errors. In particular (short) questionnaires that are missing food items contributing substantially to the nutrient intake of part of the subjects are likely to result in high reproducibility combined with low validity $(9,33)$. Relying on the reproducibility of the FFQ alone, which may be inflated by the reproducibility of the error, might therefore result in underestimation of the attenuation present in the data. For this reason, Walker and Blettner (23) have suggested to consider the reproducibility of a method as an estimate of the upper limit of the correlation of that method with a presumed underlying "true" value.

In conclusion, we have demonstrated that a single FFQ measurement characterizes dietary habits for a period of at least five years, and perhaps even for a decade. Furthermore, the ratio of within- to between-subject variance of the FFQ and the relative size of the error repeated between measurements do not seem to be as large for most nutrients as has been suggested for FFQs in general.

\section{References}

1. Block G, Hartman AM. Issues in reproducibility and validity of dietary studies. Am J Clin Nutr 1989; 50: 1133-1138.

2. Willett WC. Reproducibility and validity of food-frequency questionnaires. In: Willett WC (ed). Nutritional Epidemiology. New York/Oxford: Oxford University Press, 1990: 92-126.

3. Hankin JH, Wilkens LR, Kolonel LN, Yoshizawa CN, Validation of a quantitative diet history method in Hawaii. Am J Epidemiol 1991; 133: 616-628.

4. Willett WC, Sampson L, Stampfer MJ, Rosner B, Bain C, Witschi J, Hennekens CH, Speizer FE. Reproducibility and validity of a semiquantitative food frequency questionnaire. Am J Epidemiol 1985: 122: 51-65.

5. Jacobsen BK, Bønaa KH. The reproducibility of dietary data from a self-administered questionnaire. The Tromse study. Int J Epidemiol 1990; 19: 349.353 
6. Morabia A, Moore M, Wynder EL Reproducibility of food frequency measurements and inferences from a case-control study. Epidemiology 1990; 1: 305-310.

7. Rimm EB, Giovannucci El, Stampfer MJ, Colditz GA, Litin LB, Willett WC. Reproducibility and validity of a expanded self-administered semiquantitative food frequency questionnaire among male bealth professionals. Am J Epidemiol, 1992; 135: 1114-1126.

8. Pietinen P, Hartman AM, Haapa E, Răsînen L, Haapakoski J, Palmgren J, Albanes. D, Virtamo J, Huttunen. JK. Reproducibility and validity of dietary assessment instruments: a self-administered food use questionnaire with a portion size picture booklet. Am J Epidemiol 1988; 128: 655-666.

9. Willett W. An overview of issues related to the correction of non-differential exposure measurement error in epidemiologic studies. Stat Med 1989; 8: 1031-1040.

10. V'an den Brandı PA, Goldbohm RA, Van 't Veer P, Volovics A, Hermus RJJ, Sturmans F. A. largescale prospective cohort. study on diet and cancer in the Netherlands. J Clin Epidemiol 1990; 43: 285-295.

11. NEVO tabel. Dutch food composition table 1986-1987. The Hague, Netherlands: Voorlichtingsbureau voor de Voeding, 1986.

12. Willett W, Stampfer MJ. Total energy intake: implications for epidemiologic analyses. Am J Epidemiol 1986; 124: 17-27.

13. Van Beresteyn ECH, Van 't Hof MA, De Waard H, Dekker PR, Neeter R, Winkeldermaat H, Visser RM, Schaafsma G, Van Schiak M, Duursma SA. Design and data quality of a mixed longitudinal study to elucidate the role of dietary calcium and phosphorus on bone mineralization in pre-, peri-, and postmenopausal women. Am J Clin Nutr 1986; 43: 538-548.

14. Beaton GH. Interpretation of results from diet history studies. In: The dietary history method. Proceedings of the 2. Berlin meeting on Nutritional Epidemiology. L. Kohlmeier (ed), London: Smith-Gordon 1991.

15. Jain M, Howe GR, Harrison 1, Miller AB. A study of repeatability of dietary data over a seven-year period. Am J Epidemiol 1989; 129: 422-429.

16. Thompson FE, Metzner HI, Lamphiear DE, Hawthorne VM. Characteristics of individuals and long term reproducibility of dietary reports: the Tecumseh diet. methodology study. J Clin Epidemiol 1990; 43: 1169-1178.

17. Jensen OM, Wahrendorf J, Rosenqvist A, Geser A. The reliability of questionnaire-derived historical dietary information and temporal stability of food habits in individuals. Am J Epidemiol 1984; 120: 281-290.

18. Rohan TE, Potter JD. Retrospective assessment of dietary intake. Am J Epidemiol 1984; 120: 876-887.

19. Van Staveren WA, West CE, Hoffmans MDA et al. Comparison of contemporaneous and retrospective estimates of food consumption made by a dietary history method. Am J Epidemiol 1986; $123: 884-893$.

20. Thompson FE, Lamphiear DE, Metzner HL, Hawthorne VM, Oh MS. Reproducibility of reports of frequency of food use in the Tecumseh diet methodology study. Am J Epidemiol 1987; 125: 658-671.

21. Byers T, Marshall J, Anthony E, Fiedler R, Zielezny $M$. The reliability of dietary history from the distant past. Am J Epidemiol 1987; 125: 999-10II.

22. Wu MI, Whittemore AS, Jung DL. Errors in reported dietary intakes, II. Long-term recall. Am J Epidemiol 1988; 128: 1137-1145:

23. Walker AM, Blettner M. Comparing imperfect measures of exposure. Am J Epidemiol 1985; 121: 783-790.

24. Clayton D, Using test-retest reliability data to improve estimates of relative risk: an application of latent class; analysis. Stat Med 1985; 4: 445-455.

25. Flegal KM. Assessing dietary misclassification and its effects on relative risk (Abstract): Am J Epidemiol 1992 (in press).

26. Willett WC, Stampfer MJ, Colditz GA, Rosner BA, Hennekens CH, Speizer FE. Dietary fat and risk of breast cancer. N Engl J Med 1987;3 16: 22-8.

27. Van Beresteyn ECH, Van 't Hof MA, Van der Heiden-Winkeidermaat H, Ten Have-Witjes A, Neeter R. Evaluation of the usefulness of the cross-check dietary history method in longitudinal studies. J Chron Dis 1987; 40: 1051-1058.

28. Hunter DJ, Rimm EB, Sacks FM, Stampfer MJ, Colditz GA, Litin L.B, Willett WC. Comparison of measures of fatty acid intake by subcutaneous fat aspirate, food frequency questionnaire, and diet records in a free-living population of us men. Am J Epidemiol 1992; 135: 418-427.

29. Wu ML, Whittemore S, Jung DL. Errors in reported dietary intakes, I. Short-term recall. Am I Epidemiol 1986; 124: 826-835. 
30. Howe GR. The use of polytomous dual response data to increase power in case-control studies; an application to the association between dietary fat and breast cancer. J Chron Dis 1985; 38: 663-670.

31. De Klerk NH, Englisch DR, Armstrong BK. A review of the effects of random measurement error on relative risk estimates in epidemiological studies. Int J Epidemiol 1989; 18: 705-712.

32. Bloemberg BPM, Kromhout D, Obermann-de Boer GL, Van Kampen-Donker M. The reproducibility of dietary intake data assessed with the cross-check dietary history method. Am J Epidemiol 1989; 130: 1047-1056.

33. Engle A, Lynn L, Koury K, Boyar AP. Reproducibility and comparability of a computerized, selfadministered food frequency questionnaire. Nutr Cancer 1990; 13: 281-292. 


\title{
Chapter 7
}

\section{Predictors of toenail selenium levels in men and women*}

\author{
Piet A. van den Brandt', R. Alexandra Goldbohm ${ }^{1.2}$, Pieter van 't Veer ${ }^{2}$, Peter Bode', \\ Rudolph J.J. Hermus ${ }^{2}$, Ferd Sturmans'.
}

1) University of Limburg, Department of Epidemiology, Maastricht, the Netherlands;

2) TNO Toxicology and Nutrition Institute, Zeist, the Netherlands; 3) Interfaculty Reactor Institute, Delft University of Technology, Delft, the Netherlands

\begin{abstract}
Potential predictors of toenail selenium levels were studied In 1211 men and 1248 women aged 55-69 years. These subjects were randomly selected cohort members without prevalent cancer (other than skin) particlpating in a prospective study on diet and cancer in the Netherlands. Information on the considered potentlal predletors (gender, age, smoking, intake of dietary selenium and alcohol, Quetelet Index) was collected together with toenail specimens in 1986. The average toenall selenium concentration was significantly $(p<0.001)$ lower in men than In women: $0.547 \pm 0.126$ $\mu \mathrm{g} / \mathrm{g}$ (mean $\pm \mathrm{SD}$ ) and $0.575 \pm 0.109 \mu \mathrm{g} / \mathrm{g}$, respectively. The gender difference remained significant after adjustment for the other variables In multiple regression analyses. Age was not associated with toenall selenlum levels In men nor women. An inverse association was observed with current smoking but not with past smoking. The average toenail selenium values for male current smokers were $0.513 \pm 0.106 \mu \mathrm{g} / \mathrm{g}$ (mean $\pm S D$ ) versus $0.571 \pm 0.133 \mu \mathrm{g} / \mathrm{g}$ for male never- or ex-smokers $(\mathrm{p}<0.001)$. For women these values were $0.548 \pm 0.101$ and $0.581 \pm 0.109 \mu \mathrm{g} / \mathrm{g}$, respectlvely $(p<0.001)$. Dietary selenium intake was positively associated with toenail selenlum levels In multivariate analyses $(p<0.001$ ), but the association was weak (partlal $r=0.09$ ). Alcohol intake and Quetelet index were no significant independent predictors of toenail selenium. The observed associations had similar directions in both genders but were stronger in men.
\end{abstract}




\section{Introduction}

Several reports on the association between low tissue selenium levels and the risk of cancer and cardiovascular disease (1-4) have increased the interest in the role of selenium in disease-etiology during, the past decade. Estimation of dietary selenium intake in epidemiologic studies is considered unreliable since the selenium content of foods may vary considerably between varieties of the same type of food, depending on the soil where the food was grown. (5). Therefore, epidemiologic studies on the relation between selenium and chronic diseases often rely on biologic markers of selenium status. Especially in relation to such diseases with long latency periods as cancer, these markers should preferably reflect the long-term selenium status. Available markers include selenium levels in whole blood, serum, erythrocytes, urine, hair and nails and glutathione peroxidase activity (5-7). Of these, urinary and serum selenium levels reflect short-term changes in dietary selenium intake $(8,9)$, and it has been found that serum selenium levels may be influenced by the presence of (preclinical) disease (10). Longterm markers of selenium status include hair, erythrocytes and nails $(8,11)$, while whole blood appears to take an intermediate position in this respect between erythrocytes and plasma. (12,13).

In large-scale epidemiologic studies among thousands of subjects, markers such as erythrocytes requiring invasive sampling and specific transport and storage conditions are less attractive options. Hair and fingernails may be contaminated by seleniumcontaining anti-dandruff shampoos $(14,15)$ or environmental contamination in general and may therefore be less useful, in spite of the observed correlation between hair and blood selenium levels (3). Toenails are less prone to contamination problems in populations wearing shoes and because their surface-to-volume ratio is smaller; their usefulness as biomarker has been investigated in the last decade. Because toenails have different lengths with corresponding, age differences, toenails clipped from all toes at a single time provide a time-integrated measure of selenium intake over several months (6). Higher toenail selenium levels have been observed in subjects living in seleniferous areas as South-Dakota compared to residents of Boston or New Zealand with low selenium levels in the soil (11). Longnecker et al. (17) observed correlation coefficients of 0.91 and 0.89 for selenium levels in toenails with those in whole blood and serum, respectively, among residents of South-Dakota and Wyoming. Also, elevated selenium levels were found in toenails of subjects consuming dietary selenium supplements (16). In a recent study in South-Dakota in a population with widely varying selenium intakes, strong correlations were observed between selenium intake measurements from duplicate meal portions and selenium levels in toenails, as well as serum and whole blood (18).

The observations on the potential value and sensitivity of toenail selenium concentrations combined with feasibility considerations have stimulated the collection of toenaill clippings in epidemiologic studies (19-21). We have started a prospective cohort study on diet and cancer among men and women, that includes toenail clippings as a biologic marker of selenium status (22). Before analyzing the relationship between selenium and the risk of cancer it is important to identify potential determinants of toenail selenium levels in men and women, that may act as confounders in subsequent analyses of selenium and cancer risk. Hunter et al. (16) recently concluded that smoking, age and use of selenium supplements were predictive of toenail selenium levels among US nurses, while alcohol and dietary selenium intake were not. Swanson et al., however, did not observe a relationship with age in men and women (18). The purpose of our study was to examine whether associations between toenail selenium and 
age, smoking, alcohol, Quetelet index and selenium intake in the Netherlands do exist in women and to evaluate whether these relationships would also hold for men.

\section{Materials and methods}

\section{Subjects}

The study population is derived from an ongoing prospective cohort study on diet and cancer that was started in September 1986. The cohort $(n=120,852)$ of 55-69 year old men (48.2 percent) and women (51.8 percent) originates from 204 municipal population registries. At baseline, the cohort members completed a self-administered questionnaire on diet and potential confounding variables and also provided toenail clippings. For efficiency reasons a case-cohort approach is being used for the analysis of the cohort study' (22), requiring processing of questionnaires and toenail clippings of a random subcohort $(\mathrm{n}=3500)$ and incident cancer cases only. For the present study on potential predictors of toenail selenium we have used data of the subcohort only. Prevalent cancer cases other than skin tumours were excluded from this group, leaving 3346 subjects ( 1630 men, 1716 women). Of these, toenail clippings had been provided by 1247 men (76.5 percent) and 1322 women ( 77.0 percent). Problems with the detection of toenail selenium (interference by other elements such as calcium) occurred in 16 of these 2569 samples. An additiona! 94 specimens were excluded because the specimen weighed less than $10 \mathrm{mg}$, which would yield unreliable selenium measurements. Thus, toenail selenium data on 2459 subjects (1211 men, 1248 women) were available for analysis.

\section{Potential predictors of toenail selenium}

The considered potential predictors of toenail selenium were: gender, age, smoking habits (type of tobacco and amount smoked), alcohol consumption, Quetelet index, dietary selenium intake as calculated from food consumption and intake of selenium supplements. Information on the predictors other than gender and age was obtained from the baseline questionnaire. The food questionnaire has recently been validated (Goldbohm et al., unpublished manuscript). The dietary selenium intake was estimated by multiplying the average daily intake of foods with their selenium content. We used data on the selenium content of Dutch foods which were collected for an earlier casecontrol study on diet and breast cancer (21). Of the dietary questionnaires, about 7 percent could not be used for nutrient intake calculation because of missing or inconsistent data.

\section{Determination of toenail selenium levels}

The toenail selenium analyses were carried out by the Interfaculty Reactor Institute (IRI) at Delft University, the Netherlands. Toenails were first cleared by scratching off any debris with a quartz knife. After ultrasonic cleaning with acetone for 15 minutes, distilled water for 10 minutes and acetone for 15 minutes respectively, the specimens were freeze-dried during 15 hours to eliminate any humidity variations between runs. The selenium content of the toenails was measured by instrumental neutron activation analysis of the metastable-selenium-77 isotope. The specimens were irradiated for 17 seconds in a thermal flux of $1.2 \times 10^{13}$ neutrons. $\mathrm{s}^{-1 /} \mathrm{cm}^{-2}$. After a decay time of 20 seconds, gamına radiation of ${ }^{77 m} \mathrm{Se}$ was measured for 60 seconds. The accuracy of the method was checked by analysis off a certified Bovine liver standard (Standard Reference Material 15777a of the US National Bureau of Standards). For 26 determinations, a mean value $( \pm S D)$ of $0.70 \pm 0.04 \mu \mathrm{g} / \mathrm{g}$ selenium was observed against a certified value of $0.71 \pm 0.04 \mu \mathrm{g} / \mathrm{g}$. The precision of the method was evaluated by duplicate selenium 
measurements of specimens from 27 randomly selected subjects; the coefficient of variation was 6.6 percent.

\section{Data analyses}

Because of some skewness to the right, data on toenail selenium concentrations and dietary selenium intake were normalized with a $\log _{e}$-transformation. Selenium intake data were adjusted for energy intake by the residual method (23). The relationship between toenail selenium and its potential predictors was tested in bivariate and multivariate analyses. Analyses were carried out for men and women separately, and afterwards combined, if appropriate. First, mean toenail selenium concentrations in the various strata of the potential predictors were compared using a t-test. To investigate the influence of various potential predictors simultaneously, multiple regression analysis was employed with toenail selenium as the dependent variable and the potential predictors as independent variables. Two-sided p-values are reported throughout.

\section{Results}

Before excluding toenail specimens with weights befow $10 \mathrm{mg}$, the specimen weights ranged from 1.25 to $280.59 \mathrm{mg}$ in men and from 1.50 to $442.31 \mathrm{mg}$ in women. With the aforementioned exclusion, the average weight of the toenail specimens was $91.0 \pm 57.2$ $\mathrm{mg}$ (mean \pm SD) in men and $70.2 \pm 47.4 \mathrm{mg}$ in women. In table 1 the mean selenium levels in toenails of men and women are presented separately. Overall, men were found to have significantly $(p<0.001)$ lower mean selenium levels in toenails than women: $0.547 \pm 0.126 \mu \mathrm{g} / \mathrm{g}$ versus $0.575 \pm 0.109 \mu \mathrm{g} / \mathrm{g}$, respectively.

Table 1 also shows the associations between toenail selenium and the considered potential predictors of toenail selenium levels for each gender. In the age range studied (55-69 years) no association between toenail selenium level and age existed in men, nor in women. Smoking, on the other hand, showed a strong relationship with toenail selenium, especially among men. Current cigarette smokers have significantly $(p<0.001)$ lower toenail selenium levels than persons who never smoked and the selenium levels in men decrease with increasing amounts smoked. This relationship does not hold for women, where subjects smoking 10-19 cigarettes/day have the lowest toenail selenium concentrations. The Spearman rank correlation coefficient between (untransformed) toenail selenium and number of cigarettes smoked in the combined group of current smokers and never-smokers was -0.29 for men $(p<0.001)$ and -0.13 for women $(p<0.001)$. Men who only smoke cigars or pipe also have significantly lower toenail selenium levels than never-smokers. The corresponding Spearman correlation coefficient for these 155 subjects was $-0.20 \quad(p<0.014)$ in this case. Ex-smokers of cigarettes have somewhat lower selenium levels than never-smokers but this difference is not significant in men nor in women. When current smokers were contrasted with never or ex-smokers, the average toenail selenium values for male current smokers were $0.513 \pm 0.106 \mu \mathrm{g} / \mathrm{g}$ versus $0.571 \pm 0.133 \mu \mathrm{g} / \mathrm{g}$ for male never or ex-smokers $(p<0.001)$. For women these values were $0.548 \pm 0.101$ versus $0.581 \pm 0.109 \mu \mathrm{g} / \mathrm{g}$, respectively $(p<0.001)$.

Alcohol intake does not show a consistent relationship with toenail selenium concentration. Subjects drinking more than $30 \mathrm{~g}$ alcohol/day have lower selenium levels than non-drinkers (not statistically significant in men nor women). However, males drinking $5-14 \mathrm{~g}$ alcohol/day show somewhat higher toenail selenium levels than males drinking $1-4 \mathrm{~g}$ alcohol daily. Women drinking $1-4 \mathrm{~g}$ alcohol/day even show somewhat higher selenium levels than non-drinkers, but the levels decrease with higher akcohol consumption. 
Table 1. Toenail selenium levels $(\mu \mathrm{g} / \mathrm{g}$ ) according to various characteristics among 1211 men and 1248 women in the Netherlands, 1986.

Characteristic
Men.
Women

$n^{*} \quad$ Mean $\pm S D \quad$ p valuet $n^{*}$ Mean $\pm S D \quad$ p value

All subjects

$1211 \quad 0.547 \pm 0.126$

Age (yrs)

55-59

60-64

65-69

Smoking status

Never

Only cigar/pipe

Ex-cigarette

Current cigarette

$1-9 /$ day
$10-19 /$ day
$\geq 20 /$ day

Alcohol intake (g/day)

$$
\begin{array}{r}
0 \\
1-4 \\
5-14 \\
15-29 \\
\geq 30
\end{array}
$$

Quetelet index $\left(\mathrm{kg} / \mathrm{m}^{2}\right)$
$<20$
20-24
25-29
$\geq 30$

\section{0}

235

314

261

159

$0.565 \pm 0.193$
$0.543 \pm 0.090$
$0.556 \pm 0.126$
$0.538 \pm 0.120$
$0.538 \pm 0.096$

$350.516 \pm 0.106$

579

513

47

$0.550 \pm 0.140$

$0.545 \pm 0.104$

$0.551 \pm 0.086$

(2)

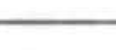

$1248 \quad 0.575 \pm 0.109$

Dietary Se ( $\mu$ g/day), quintiles (energy-adjusted)
1 ( $\leq 47.5)$
$2(>47.5-\leq 53.3)$
$3(>53.3-\leq 59.1)$
$4(>59.1-\leq 66.7)$
$5(>66.7)$

\section{3}

227

216

232

220

$0.529 \pm 0.095$
$0.548 \pm 0.169$
$0.543 \pm 0.126$
$0.561 \pm 0.119$
$0.562 \pm 0.116$

-
0.167
0.282
0.001
0.001

$\begin{array}{ll}213 & 0.550 \pm 0.097 \\ 235 & 0.581 \pm 0.106 \\ 243 & 0.585 \pm 0.129 \\ 237 & 0.575 \pm 0.104 \\ 250 & 0.577 \pm 0.096\end{array}$

\subsection{1}

0.313

$<0.001$

$<0.001$

$<0.001$

0.271
0.776
0.072
0.134

366

$404 \quad 0.580+0.114$

$209 \quad 0.572 \pm 0.100$

$103 \quad 0.570 \pm 0.106$

$45 \quad 0.557 \pm 0.082$

0.438

0.908

0.821

0.403

$$
\begin{aligned}
& 0.150 \\
& -\ddagger \\
& 0.733 \\
& 0.558
\end{aligned}
$$

$-\ddagger$

0.626

\footnotetext{
- Due to missing questionnaire data, numbers may not add up to 1211 and 1248 , respectively.

$\dagger$ T-test between strata, based on In-transformed toenail selenium levels.

$\ddagger$ Reference category.
}

Subjects with a Quetelet index less than $20 \mathrm{~kg} / \mathrm{m}^{2}$ exhibit lower toenail selenium levels in men and women than in the referent category $20-25 \mathrm{~kg} / \mathrm{m}^{2}$, although the differences are not significant. Although women with a Quetelet index between 25-29 have significantly increased toenail selenium levels compared to the referent category, the levels are decreased again in the obese women $\left(\mathrm{QI} \geq 30 \mathrm{~kg} / \mathrm{m}^{2}\right)$. In men the toenail selenium levels are comparable in the upper three Quetelet index categories. 
Selenium supplement use was not reported by any of the subjects studied; therefore its relationship with toenail selenium was not evaluated. Since the estimated selenium intake was positively correlated with total energy intake $(r=0.58)$, the relationship between toenail selenium and dietary selenium was evaluated after adjusting the latter for energy intake. After dividing the energy-adjusted selenium intake into quintiles (for men and women combined) there was a positive trend with toenail selenium levels. For men, the mean toenail selenium levels in the upper two quintiles of intake were significantly higher than in the lowest quintile. For women, the mean toenail selenium levels in the second and third quintile were also significantly higher than in the bottom quintile but the levels in the upper two quintiles were somewhat lower than in the second and third quintile. The Spearman correlation coefficient between toenail selenium and energy-adjusted dietary selenium intake was $0.11(\mathrm{p}<0.001)$.

To investigate whether the lower toenail selenium levels in smokers might possibly be due to a decreased intake of selenium we also calculated the average energyadjusted selenium intake according to smoking habits and tested the differences in both men and women (table 2 ). The results in table 2 indicate that male current smokers indeed consume less selenium per day (after adjustment for energy intake) than those who never smoked; the difference increases with increasing amount smoked and is significant for those smoking 20 cigarettes/day or more. In women, no significant differences were observed although heavy smokers consume less selenium. Table 2 also shows the mean energy-adjusted selenium intake values in men and women without regard to smoking status. The mean selenium intake in men is significantly lower $(\mathrm{p}=0.003$ ) than in women: $57.0 \pm 12.7 \mu \mathrm{g} /$ day and $58.7 \pm 14.0 \mu \mathrm{g} /$ day, respectively.

Table 2. Mean ( \pm SD) daily energy-adjusted intake of selenium $(\mu g)$ in men and women separately according to smoking status.

Smoking status Men Women
n
Mean $\pm S D$
p value*
Mean $\pm S D$
$\mathrm{p}$ value*

$\begin{array}{lrlllll}\text { All subjocts } & 1148 & 57.0 \pm 12.7 & & 1178 & 58.7 \pm 14.0 & \\ & & & & & & \\ \text { Never snoked } & 113 & 58.1 \pm 12.9 & -\dagger & 701 & 58.4 \pm 14.0 & -\dagger \\ \text { Only cigar/pipe } & 43 & 58.6 \pm 15.0 & 0.874 & & & \\ \text { Ex-cigarette } & 613 & 58.0 \pm 12.6 & 0.972 & 240 & 60.0 \pm 13.2 & 0.096 \\ \begin{array}{l}\text { Current cigarctte } \\ \quad\end{array} & 54 & 56.8 \pm 11.8 & 0.586 & 73 & 60.4 \pm 17.1 & 0.458 \\ \quad \text { 1-9/day } & 138 & 55.9 \pm 13.2 & 0.150 & 90 & 58.1 \pm 13.8 & 0.788 \\ \text { 201+/day } & 142 & 54.3 \pm 12.2 & 0.014 & 62 & 55.3 \pm 14.3 & 0.065\end{array}$

- T-test based on ln-transformed toenail selenium values.

† Reference category.

The results of both bivariate regression and multiple regression analyses, where the effects of several predictors are controlled simultaneously, are shown in table 3 . Because the previous analyses in both genders had not revealed any large differences between men and women in the associations with potential predictors, the regression analyses were carried out for the combined group. The multivariate analysis yielded a significant effect of gender while no effect of age was observed. The negative association between smoking on toenail selenium levels remained significant in the 
regression model although not for subjects who only smoke cigars or pipe. Also, the energy-adjusted selenium intake showed an independent positive association with toenail selenium levels in this population. The partial correlation coefficient between the two variables was 0.09 , indicating a weak relationship. Although the effect is in the anticipated direction, the significance of it $(p<0.001)$ is merely the result of the large number of individuals studied. The observed regression coefficient of 0.082 for the $\log _{e}$ transformed values in the multivariate model implies, for example, a 4 percent increase in toenail selenium level when the median of the top quintile of selenium intake (73.8 $\mu \mathrm{g} /$ day) is contrasted with the median of the bottom quintile of intake $(43.3 \mu \mathrm{g} /$ day). Alcohol intake and Quetelet index were no significant predictors of toenail selenium in this model. Together, the independent predictors gender, smoking habits and selenium intake explained 7 percent of the variance in toenail selenium levels in this population.

Regression analyses conducted for men and women separately (results not shown here) revealed similar directions of the associations with smoking and selenium intake in both genders, although the associations were somewhat stronger in men.

Table 3. Predictors of toenail selenium levels $(\mu \mathrm{g} / \mathrm{g}$, ln-transformed) in bivariate and multiple regression analysis in 2459 men and women, the Netherlands, 1986.

$$
\text { Bivariate regression Multiple regression }
$$

$\dot{B} \operatorname{se}(\hat{B}) \quad$ p value $\dot{B} \quad \operatorname{se}(\dot{B}) \quad p$ value

Intercept

Gender $(M=0, F=1)$

Age (10 years)

Smoking habits*

Only cigar/pipe

1- 9 cigts/day

10-19 cigts/day

$\geq 20 \mathrm{cigts} /$ day

Alcohol intake $(\mathrm{g} /$ day $) \dagger$

$<15$

$\geq 15$

Quetelet index $\left(5 \mathrm{~kg} / \mathrm{m}^{2}\right)$

Selenium intake ( $\mathrm{ln} \mu \mathrm{g} /$ day)

(energy-adjusted)

\begin{tabular}{rrrrrr} 
& & \multicolumn{5}{c}{-0.858} \\
0.051 & 0.008 & $<0.001$ & 0.034 & 0.009 & $<0.001$ \\
0.005 & 0.009 & 0.571 & -0.010 & 0.010 & 0.308 \\
& & & & & \\
-0.074 & 0.029 & 0.011 & -0.043 & 0.030 & 0.151 \\
-0.053 & 0.017 & 0.002 & -0.043 & 0.017 & 0.013 \\
-0.109 & 0.013 & $<0.001$ & -0.106 & 0.014 & $<0.001$ \\
-0.116 & 0.014 & $<0.001$ & -0.093 & 0.014 & $<0.001$ \\
& & & & & \\
-0.007 & 0.010 & 0.493 & -0.006 & 0.010 & 0.532 \\
-0.041 & 0.012 & $<0.001$ & -0.011 & 0.012 & 0.343 \\
0.015 & 0.006 & 0.020 & 0.001 & 0.007 & 0.870 \\
0.101 & 0.018 & $<0.001$ & 0.082 & 0.018 & $<0.001$
\end{tabular}

$\mathrm{R}^{2}=0.07 ;$ adjusted $\mathrm{R}^{2}=0.07$

- Dummy variables (coded as 0,1 ) were introduced for the smoking categories, with never- or ex-smokers as baseline category.

$\uparrow$ Dummy variables were introduced for the drinking categories, with nondrinkers as baseline category. 


\section{Discussion}

The average toenail selenium values that we observed among women $(0.58 \mu \mathrm{g} / \mathrm{g})$ are somewhat lower than other recent estimates $(0.65 \mu \mathrm{g} / \mathrm{g})$ from the Netherlands (21), but the estimates of selenium intake in both studies were virtually similar, using the same food tables. The difference in toenail levels might be due to differences in analytic conditions, used in different nuclear research reactors. Among US nurses toenail selenium levels around $0.80 \mu \mathrm{g} / \mathrm{g}$ were observed (16), whereas substantially higher values (averaging $1.17 \mu \mathrm{g} / \mathrm{g}$ ) were found among residents of a seleniferous area in South-Dakota (18). Similar values were found by Morris et al. (11) who also observed an average value of $0.26 \mu \mathrm{g} / \mathrm{g}$ for residents of New Zealand. In Greece values around $0.54 \mu \mathrm{g} / \mathrm{g}$ have been observed in fingernails $(24)$. The fact that men have lower toenail selenium levels than women was also observed by Swanson et al. (18). Other studies did not show a clear gender difference regarding fingernail (24), whole blood or plasma selenium levels (25). However, Lloyd et al. (25) did find significantly lower selenium values in erythrocytes among men.

Age was not a predictor of toenail selenium in our study. Various investigators have found an inverse association between selenium status parameters and age $(16,25,26)$ but this observation is not consistent $(18,24,27)$. In the study of Dickson and Tomlinson (26), the inverse association between serum selenium and age was restricted to subjects under 55 years of age, while Lloyd et al. (25) only observed lower levels in persons 56 years or older, and Bratakos et al. (24) noted an inverse trend only in subjects 40 years or older. The absence of an age effect in our study may also be due to the limited age range in our cohort population (55-69 years).

Smoking was an independent predictor of lowered toenail selenium levels in both men and women. In general, a negative dose-response relationship existed with the amount smoked, as was observed earlier in women (16). Swanson et al. (18) also observed a strong negative association of smoking with toenail selenium levels, but not with serum or whole blood selenium. Negative relationships with smoking have been observed for whole blood and serum selenium (28). Lloyd et al. (25) found significantly decreased levels of selenium in whole blood, plasma and erythrocytes among smoking men, but for smoking women only in plasma for those who drank alcohol daily. This is in accordance with our observation that the smoking effect is more pronounced in men than in women. It is unclear whether smoking induces increased selenium utilization (e.g. as active site of glutathione peroxidase) or influences selenium incorporation in nail keratin. Our data indicate that current smokers have lower energy-adjusted selenium intakes compared to never-smokers, but in the multiple regression analysis smoking remained an independent predictor. Swanson et al. (18) also observed significantly lower selenium intakes among smokers although no adjustment was made for energy intake. We also compared selenium intakes of smokers and nonsmokers without adjustment for energy intake; the unadjusted intakes in ex- or in current smokers were not significantly lower than in those who never smoked.

Our estimates of selenium intake are substantially lower than the intakes measured by Swanson et al. (18) among South-Dakota residents $(174 \mu \mathrm{g} /$ day) using the duplicate portion technique. These investigators observed a very strong independent effect of dietary selenium on toenail selenium levels. Our intake estimates are also lower than the intakes estimated by Hunter et al. (16) with a questionnaire among US nurses. The estimation of dietary selenium intake based on questionnaires or interviews is difficult because of large variations in selenium contents within varieties of the same food, depending on soil conditions. When a single selenium food table is used, the estimation problem is likely to aggravate if a study is conducted in a country as the US as opposed 
to the Netherlands (16). Although we observed a positive association between selenium intake and toenail levels, in contrast to Hunter et al. (16), the partial correlation coefficient between dietary and toenail selenium was still only 0.09 in our study, indicating a weak positive relationship which reached only significance due to the large numbers of subjects studied. We could not evaluate the influence of dietary methionine on the toenail selenium levels (which was recently found to be a mediating factor in the deposition of selenium in nails of rats (29)), because information on methionine intake was not available.

Although in bivariate regression analysis of men and women combined, subjects drinking $15 \mathrm{~g}$ of alcohol or more per day showed significantly lower toenail selenium levels, alcobol intake was not an independent predictor of toenail selenium in multivariate analyses with smoking in the model. Earlier studies have revealed decreased serum selenium levels among alcoholics $(30,31)$. The absence of an alcohol effect in the dose range we have studied is in agreement with other studies using toenail selenium (16), or whole blood or erythrocyte selenium (25). With regard to plasma selenium, Lloyd et al. (25) observed decreased levels in non-smoking men who were daily drinkers compared to non- and weekend drinkers, but not in women.

In bivariate analyses there was an indication that Quetelet index is positively associated with toenail selenium levels, but it was no independent predictor in multiple regression analyses. Swanson et al. (18) suggested that controlling for differences in lean body mass instead of weight (or relative weight) would diminish the effect of gender on toenail selenium levels, because of the storage of selenium in muscle tissue $(32,33)$. This adjustment is difficult in practice, however, because data on lean body mass are typically unavailable. The present result does not support a need to control for Quetelet index as a confounder in studies on selenium and obesity-associated diseases.

In conclusion, smoking was the most important predictor of toenail selenium levels in both men and women. The inverse relationship with smoking appeared to be stronger in men than in women. Gender itself was also an independent predictor of toenail selenium, with men showing lower values than women. Dietary selenium intake (excluding supplements) showed a significantly weak positive association with toenail selenium levels, despite the well-known lack of reliability of questionnaire-based assessment of selenium intake (5). Age, alcohol intake and Quetelet index were no independent predictors of toenail selenium levels. In any epidemiologic analysis relating toenail selenium to risk of smoking associated diseases, adjustment for smoking habits is indicated.

\section{References}

1. Willell WC, Morris. JS, Pressel S, et al. Prediagnostic serum selenium and risk of cancer. Lancet 1983; ii: $130 \cdot 134$.

2. Keshan Disease. Research Group. Observations on effect of sodium selenite in prevention of Keshan Disease. Chin Med J 1979; 92: 471-476.

3. Chen $X$, Yang G, Chen $J$, et al. Studies on the relations of selenium and Keshan Disease. Biol Trace Elem Res 1980; 2: 91-107.

4. Schrauzer GN, White DA, Schneider CJ. Cancer mortality correlation studies-111: Statistical associations with dietary selenium intakes. Bioinorganic Chennistry 1977: 7: 23.34.

5. Levander OA. The need for measures of selenium stalus. J Am Coll Toxicol 1986; 5: $37-44$

6. Willett W. Nutritional Epidemiology. New York: Oxford University Press, 1990,

7. Hulka BS, Wileosky' TC, Griffith JD. Biological markers in epidemiology. New York: Oxford University Press, 1990.

8. Levandier OA. Considerations; on the assessment of selenium status. Fed Proc 1985; 44: 2579-2583.

9. Robinson JR, Robinson MF, Levander $\mathrm{OA}$, et al. Urinary excretion of selenium by New Zealand and North American human subjects on differing intakes. Am I Clin Nutr 1985; 41: 1023-1031. 
10. Broghamer WL, McConnell KP, Blotcky AL. Relationship between serum selenium levels and patients with carcinoma. Cancer 1976; 37: 1384-1388.

11. Morris JS, Stampfer MJ, Willett W. Dietary selenium in humans; toenails as an indicator. Biol Trace Elem Res 1983; 5: 529-537.

12. Thompson CD, Robinson MF, Campbell DR, et al. Effect of prolonged supplementation with daily supplements of selenomethionine and sodium selenite on glutathione peroxidase activity in blood of New Zealand residents. Am J Clin Nutr 1982; 36: 24-31.

13. Taylor PR, Longnecker MP, Levander $\mathrm{OA}$, et al. Seasonal variation in selenium (Se) status among free-living persons in South-Dakota. Fed Proc 1987; 46: 882.

14. Gordus A. Factors affecting the trace-metal content of human hair. J Radioanal Chem 1973; 15: 229.243.

15. Davies TS. Hair analysis and selenium shampoos. Lancet 1982; ii: 935.

16. Hunter DJ, Morris JS, Chute CG, et al. Predictors of selenium concentration in human toenails. Am J Eepidemiol 1990; 132: 114-122.

17. Longnecker MP, Taylor PR, Levander OA, et al. Selenium in diet, blood, and toenails in relation to human health in a seleniferous area. Am J Clin Nutr 1991; 53: 1288-1294.

18. Swanson CA, Longnecker MP, Veillon C, et al. Selenium intake, age, gender, and smoking in relation to indices of selenium status of adults residing in a seleniferous area. Am J Clin Nutr 1990; 52: 858-862.

19. Hunter DJ, Morris JS, Stampfer MJ, et al. A prospective study of selenium status and breast cancer risk. JAMA 1990; 264: 1128-1131.

20. Van Noord PAH, Colette HJA, Maas MJ, et al. Selenium levels in nails of premenopausal breast cancer patients assessed prediagnostically in a cohort-nested case-referent study among women screened in the DOM project. Int I Epidemiol 1987; 16: 318-322.

21. Van 't Veer P, Van der Wielen PJ, Kok FJ, et al. Selenium in diet, blood, and toenails in relation to breast cancer: a case-control study. Am J Epidemiol 1990; 131: 987-994.

22. Van den Brandt PA, Goldbohm RA, Van 'i Veer P, et al. A large-scale prospective cohort study on diet and cancer in The Netherlands. J Clin Epidemiol 1990; 43: 285-295.

23. Willett W, Stampfer MJ. Total energy intake: implications for epidemiologic analyses. Am J Epidemiol 1986; 124: 17-27.

24. Bratakos MS, Kanaki HC, Vasiliou Waite A, et al. The nutritional selenium status of bealthy Greeks. Sci Total Environ 1990; 91: 161-176.

25. Lloyd B, Lloyd RS, Clayton BE. Effect of smoking, alcohol, and other factors on the selenium status of a healthy population. J Epidemiol Community Health 1983; 37: 213-217.

26. Dickson RC, Tomlinson RH. Selenium in blood and human tissues. Clin Chim Acta 1967; 16: $311-321$.

27. McAdan PA, Smith DK, Feldman EB, et al. Effect of age, sex, and race on selenium status of healthy residents of Augusta, Georgia. Biol Trace Elem Res 1984: 6: 3-9.

28. Ellis N, Lloyd B, Loyd RS, et al. Selenium and vitanin $\mathrm{E}$ in relation to risk factors for coronary heart disease. J Clin Pathol 1984: 37: 200-206.

29. Salbe AD, Levander OA. Effect of various dietary factors on the deposition of selenium in the hair and nails of rats. J Nutr 1990; 120: 200-206.

30. Korpela H, Kumpulainen J, Luoma PV, et al. Decreased serum selenium in alcoholies as related to liver structure and function. An J Clin Nutr 1985: 42: 147-151.

31. Aaseth J, Thomassen $\mathrm{Y}$, Alexander $\mathrm{J}$, et al. Decreased serum selenium in alcoholic cirrhosis. $\mathrm{N}$ Engl J Med 1980: 303: 944-945.

32. Behne D, Wolters W. Distribution of selenium and glutathione peroxidase in the rat. J Nutr 1983; 113: 456-61.

33. Schroeder HA, Frost DV, Balassa JJ. Essential trace metals in man: selenium. J Chron Dis 1970; $23: 227-243$ : 


\title{
Assessment of Dietary Nitrate Intake by a Self-Administered Questionnaire and by Overnight Urinary Measurement
}

\author{
PIET A VAN DEN BRANDT. " WALTER C WILLETT * AND STEVEN R TANNENBAUM
}

\begin{abstract}
Van den Brandt P A (Department of Epidemiology. University of Limburg Maastricht, The Netheriands) Willett W C and Tannenbaum S R. Assessment of dietary nitrate intake by a self-administered questionnaire and by overnight urinary measurement. International Journal of Epidemiology, 1989, 18: 852-857.

The relationship between dietary intake and urinary excretion of nitrate was investigated among 35 male and 24 femaie graduate students in Boston. The dietary assessment method consisted of a seif-administered semi-quantitative food frequency questionnaire currently used for large-scale epidemiological studies. Calculated mean daily nitrate intake was $1.83 \mathrm{mmol}$ for men and $2.96 \mathrm{mmol}$ for women; broccoli and green leafy vegetables accounted for $60 \%$ of the total. Urinary measurements involved two overnight specimens with a mean collection time of approximately 13 hours. The ratio of intra-to-inter individual variance in urinary nitrate excretion (lambda) was 1.87. The simple correlation coefficient between intake and excretion of nitrate was found to be 0.20 ; after correction for the within-person variation by using lambda, the correlation coefficient was 0.28 . Adjustment for gender, age and Quetelet's Index in multiple regression analyses resulted in a partial correlation coefficient between nitrate intake and excretion of 0.37 ( $p=0.005)$. Correction for within-person variation in urinary excretion increased this partial correiation coefficient between intake and excretion to $0.59(95 \% \mathrm{Cl}=0.03$ to 0.87$)$. These data suggest that a self-administered questionnaire may provide useful information on usual nitrate intake, and indicate the need to pursue this possibility further.
\end{abstract}

Increasing interest in relationships between long-term dietary intake and the occurrence of chronic disease has stimulated the development and evaluation of methods to measure dietary factors among large groups of individuals. Methods based on questionnaires' or biochemical measurements may both be useful, depending on the parameter being assessed and on the practical constraints imposed by the particular study design. For any method it will be important to evaluate the reproducibility and validity of the measurement. Since long-term intake is important in most epidemiological hypotheses, interview or biochemical parameters that reflect intake over a short period (such as a single day) may be of limited use, even though highly accurate for that short interval.

- Department of Epidemiology. Univeruty of Limburg. Maastricht. The Netherlands.

* Departments of Epidemiology and Nutrition. Harvard School of Public Health and Channing Laboratory and Brigham and Women * Hospital. Boston. USA

t Department of Applied Biologeal Scaences. Massachusetis Institute of Technology, Cambrige. USA.

Reprint requests. Dr Willett, Channing Laboratory, 180 Longwood Avenue. Boston. MA 02115, USA
When possible, the use of biochemical measurements to validate dietary questionnaires is appealing since the sources of error should be largely independent. In a previous study' we evaluated the capacity of our semi-quantitative food frequency questionnaire to measure vitamin $\mathrm{E}$ and carotenoid intake using plasma levels of these nutrients for comparison. However, the use of biochemical measures for this purpose is fre. quently limited because many are insensitive to dietary intake over much of the dose-response range, or are highly variable from day to day. Urinary nitrate has been proposed as an estimate of the dietary nitrate intake, after the observation that $65-70 \%$ of ingested nitrate is excreted in the urine during the following 24 hours and less than $1 \%$ in faeces. ${ }^{+}$

Nitrate has been hypothesized to play a role in the aetiology of certain gastrointestinal cancers, notably gastric cancer. ${ }^{5-4}$ Nitrate may be converted into nitrite in foods, in the stomach" and in the oral cavity; ${ }^{.10}$ nitrate and nitrite can react with secondary amines or amides to form $\mathrm{N}$-nitroso compounds. ${ }^{11.12}$ The relation of nitrate intake with cancer risk has been investigated in various epidemiological studies. ${ }^{\text {is }}$ is Attention has also been given to the cancer risk associated with nitrate in drink- 
ing water because of the increasing use of nitrogenous fertilizers. ${ }^{17}$ In none of these studies was evidence found to support a positive association.

We therefore assessed the within-person variability of timed overnight urinary nitrate excretion measurements and the influence of demographic and other factors on these levels. We then used these urinary measures to evaluate questionnaire estimates of nitrate intake. Finally, we evaluated the effects of within-person variation and other variables on the association between questionnaire estimates of intake and urinary measurement.

\section{MATERIALS AND METHODS \\ Subjects}

In April 1984 we invited a random sample of the studen! population of the Harvard School of Public Health to participate in this study. The sample consisted mainly of North American and European students. but also included four Japanese students. Sixty-one students agreed to participate, but two failed to complete all procedures. Hence, our analyses are based on 59 subjects consisting of 35 males and 24 females, age 30.3 (1 $\mathrm{SD} \pm 4.5)$ and $28.9( \pm 6.5)$ years respectively. Subiects were unaware of the fact that the investigation concerned the consumption and excretion of nitrate. since that might have interfered with their intake. The procedures used in this study were approved by the Cornmittee on the Use of Human Subjects at the Harvard School of Public Health, and all individuais signed an informed consent form.

\section{Overall Design}

Participants were asked to collect timed, overnight urine specimens on two occasions, separated by two weeks. To impose minimal inconvenience on the subjects, they were asked to start the urine collection after they had arrived at home in the evening and to continue until the next morning, recording the exact time of starting and stopping. We appreciated that the overnight specimens would be less optimal than full 24-hour collections; however, we wished to evaluate a method that might be feasible on a much larger scale. In this way urine samples covering a period of approximately 13 hours were obtained. The dietary questionnaire was completed at the time the first urine specimen was obtained.

\section{Dietary Questionnaire}

The semi-quantitative food frequency questionnaire we employed is a questionnaire that is being used in a variety of epidemiological studies. Earlier versions of this form were validated previously with respect to the intake of carotene, retinol, and vitamin $\mathrm{E}^{3}$ as well as other nutrients. The current self-administered ques. tionnaire consists of 120 specified foods; the participant is asked how often, on average, a specified quantity of each food was consumed over the past year. Nine responses, are possible, ranging from never to six or more times a day.

As described, elsewhere, nutrient scores were computed by summing the products of the frequency and nutrient composition of the specified serving size for each food. ${ }^{1}$ Food composition values for nitrate were derived from a report of the National Research Council on the health effects of nitrate, ${ }^{\circ}$ and a compilation by White. "For some food items the nitrate content was derived by extrapolation, since published data were not available. Nitrate from drinking water was not included in our calculation; however, the concentration of this ion in the Boston municipal water supply is less than five parts per million. Assuming an average intake of one litre of water per day, the intake from this source would be less than $0.05 \mathrm{mmol} / 24$ hours (which is less than $2 \%$ of intake from food sources).

\section{Urine Collection}

Timed overnight urine specimens were collected in two litre plastic bottles containing $50 \mathrm{ml}$ of $3 \% \mathrm{HCl}$ solution as a bacteriostatic agent. On the morning that the collection was completed, four $10 \mathrm{ml}$ aliquots of urine were taken and immediately frozen at $-20^{\circ} \mathrm{C}$ for chemical analysis. At both collection periods subjects were asked about any infections they might have had at that time. since this might influence the nitrate content of the urine (D A Wagner, personal communication).

\section{Chernical Analysis}

Urinary nitrate concentrations were determined via reduction with a high-pressure cadmium column as described by Giteen et al. ${ }^{10}$ Creatinine concentration was measured by flame photometry.

\section{Statistical Analyses}

Statistica! analyses were carried out using the BMDP statistical package. ${ }^{30}$ Urinary values were expressed as excretion rates per hour; in the analyses comparing dietary and urinary values, the mean of the excretion rates for the rwo collection periods was used as the dependent variable. Highly skewed variables were logarithmically transformed to meet normality assumptions. Dietary intake: and excretion. values were tested for genderspecific differences using Student's t-test.

Analysis of variance was performed on the two repeated urinary measurements per person to examine the components of variability in nitrate excretion as 
described by Beaton. 2In this way estimates of the interindividual $\left(\mathrm{s}_{\mathrm{b}}{ }^{2}\right)$ and intra-individual variance $\left(\mathrm{s}_{*}{ }^{2}\right)$ were computed. The intra-individual variance is composed. of the biological intra-individual variation (which may be largely due to daily variation in diet) as well as random measurement errors in the urinary values. The ratio of the intra-individual to the inter-individual variance components (lambda) provides an indication of the reproducibility of the urinary excretion values. Lambda can also be used to determine the degree of attenuation in the estimated correlation or regression coefficient describing the relationship between the dietary and urinary variables that is due to within-person variation. Conversely, these coefficients can be corrected for within-person variation by using lambda. In this application, the corrected correlation coefficient can be thought of as the correlation that would describe the relationship between the questionnaire measurement and an infinite number of urine specimens per subject. The relationship between the true and observed coefficient is as follows, ${ }^{2.21}$

$$
\mathbf{r}_{\mathrm{f}}=\mathbf{r}_{\mathrm{o}} \sqrt{(1+\lambda / \mathrm{k})}
$$

where $r_{o}=$ observed correlation coefficient

$r_{1}=$ true correlation coefficient

$k=$ number of measurements per person

$\lambda=$ ratio of within-person variance to betweenperson variance.

Confidence intervals for the corrected correlation coefficient were also computed. ${ }^{22}$.

We first computed simple correlation coefficients to compare nitrate intake and urinary output. In the final analyses we utilized multiple regression to assess the influence of several predictors of the urinary excretion rate simultaneously. Statistical significance is expressed as two-sided p-values throughout the text.

\section{RESULTS}

\section{General Description}

The daily intake of nitrate as estimated by the questionnaire was lower in males $(\mathbf{i} .83 \pm 0.79 \mathrm{mmol})$ than in

TAale 1 Overnight urnarv excretion of nitrate and creatinine among 59 men and women (mean \pm 1 SD). Date were collecied in Boston. USA during/1984

\begin{tabular}{|c|c|c|c|}
\hline Variable & $\begin{array}{c}\text { Men } \\
(n=35)\end{array}$ & $\begin{array}{l}\text { Women } \\
(n=24)\end{array}$ & p-value \\
\hline Time of collection (hrs) & $13.0 \div 2.3$ & $12.5=1.8$ & \\
\hline Nitrate (mmolhr) & $0.08 \pm 0.04$ & $0.05=0.02$ & $<0.001$ \\
\hline (umol/hr/kg) & $1.15=0.67$ & $0.81=0.42$ & 0.011 \\
\hline Creatinune (mghr) & $676=94$ & $42.8=92$ & $<0.001$ \\
\hline (mg hrikg) & $098 \pm 010$ & $0.78=0.17$ & $<0,001$ \\
\hline
\end{tabular}

females $(2.96 \pm 2.05 \mathrm{mmol})$; however, this difference did not reach statistical significance. Broccoli, spinach, other greens and lettuce accounted for $60 \%$ of the calculated nitrate intake among the 59 subjects; each of these items was reported more frequently by women.

The excretion rate of nitrate was found to be higher among men than among women (see Table 1). Thus. men were estimated to have a lower intake of nitrate. although their excretion was higher, whether expressed as mmol/hr or $\mu \mathrm{mol} / \mathrm{hr} / \mathrm{kg}$. If we assume that overnight urine samples are representative of 24-hour excretion. then the proportion of ingested nitrate that would be excreted was 1.04 in males and only 0.37 in females. Estimates of the within-person and between-person variances, as well as the ratio of these two variance components, are given in Table 2 . The variance ratio for nitrate is 1.87 , while that for creatinine is 0.36 .

\section{Relationship Between Intake and Excretion of Nitrate}

The Pearson correlation coefficient between nitrate intake and excretion per hour was 0.20 ( $p>0.05$ ). Although this simple correlation was not statistically significant, it has not been corrected for variables that might influence (ie confound) the relationship between intake and excre!ion rate. In Table 3 Pearson moment correlation coefficients are reported between potential confounders and the excretion rate.

As noted above, men had a higher excretion rate than women. Quetelet's Index (weightheight ${ }^{2}$ ) and age were positively correlated with nitrate excretion. Caloric intake, as well as the intake of most macronutrients (not presented), did not appear to be strongly related to the excretion rate. Subsequent regression analyses were carried out with and without adjustment for caloric intake. Since the resuits were not materially different and nitrate-rich foods in general contain few calories, caloric intake was left out of the regression model presented. We did not find an effect of reported infection on the measurement of nitrate excretion.

Adjusting the relationship between intake and excretion for these potentially confounding variables resulted in higher partial' correlation coefficients in some instances (Table 4). Allowing for gender or Quetelet's Index changed the partial correlation coefficient between intake and excretion of nitrate substantially.

Multiple regression analysis was performed to control for the effects of several predictors simultaneously. The results of both univariate regression and multiple regression analyses for nitrate are shown in Table 5 . Only nitrate intake. gender and Quetelet's Index were significantly associated with nitrate excretion in the 
TABu: 2 Variation in unnary excretion levels of nitrute and crratinine in 59 men and women. Boston, 1954

\begin{tabular}{cccc}
\hline Varable & $\begin{array}{c}\text { Within-person } \\
\text { variance }\end{array}$ & $\begin{array}{c}\text { Between-pervon } \\
\text { varuance }\end{array}$ & $\begin{array}{c}\text { Variance ratio } \\
\text { (lambda) }\end{array}$ \\
\hline Nitrate (umolhr) & 16040 & 859.6 & 1.87 \\
Creatinine (mghr) & 709 & 1993 & 0.36 \\
\hline
\end{tabular}

multiple regression model. Inclusion of age did not affect the estimated regression coefficients, nor $R^{2}$. The partial correlation coefficient between nitrate intake and excretion after controlling for Quetelet's Index, gender and age was 0.37 . Since men reported a lower nitrate intake, but were observed to have a higher excretion of the ion, we also tested for possible interaction between gender and nitrate intake: no evidence was found for a statistically significant interaction.

As suggested by Beaton et $a l^{2}$ and Liu et $a l^{21}$ we used the ratio of within-to-between-person variance to adjust the correlation coefficient between nitrate intake and excretion. Utilizing the observed simple correlation coefficient from Table 4 , and the values for lambda from Table 2, the corrected coefficient adjusted for within-person variation was $0.28(95 \%$ confidence interva! $(\mathrm{CI})=-0.09,0.58)$. This simple correlation. however, does not reflect the effects of variables that would normally be controlled in any epidemiological analysis, such as age and sex. or that influenced the relation between intake and excretion in this data set. such as Quetelet's Index. We therefore also calculated the value of lambda for nitrate excretion after adjusting each individual level for the variables in Table 5 (adjusted levels were computed as the residuals of the excretion values regressed on the predictor variables). As expected, the value of iambda increased from 1.87 to 3.13 since sources of inter-individual variation were removed while the intra-individual variation remained unchanged. This adjusted value of lambda was then used to correct the partial correlation from Table 5 . which represents the correlation between the adjusted excretion and intake levels. Although the correlation coefficient increased substantially with this correction (0.59), the associated $95 \%$ confidence interval was wide $(0.03,0.87)$.

\section{DISCUSSION}

In this population of graduate students, we observed a moderately high within-person variability in the overnight urinary excretion of nitrate. The intra-to-inter individual variance ratio (lambda) for nitrate excretion was found to be 1.87. Although this is less than the lambda-value reported for sodium of $3.20,{ }^{23}$ the intra- individual variation in nitrate excretion remains considerable. The use of a single overnight specimen will therefore be of limited utility for characterizing an individual's long-term intake or excretion. of this ion. although it may be useful for comparing populations. In simple bivariate analyses, nitrate intake based on a selfadministered dietary questionnaire wasi only weakly correlated with excretion measured by the average of two overnight urine samples. However, adjustment for additional variables in multiple regression analysis increased the association between intake and excretion, and further correction for within-person variability in urinary excretion suggested that: the questionnaire may actually provide reasonable discrimination of individual intakes of nitrate.

The predictive value of overnight urine specimens was studied, among others, by Watson and Langford, ${ }^{24}$ who compared excretion rates in specimens collected overnight and during the full 24 hours. They reported correlations for sodium and potassium excretion between the overnight and 24-hour excretion rates of 0.76 and 0.73 sespectively. While in their experiment the mean duration of overnight collection was only 7.9 hours, in our study this was almost 13 hours. Our specimens would therefore be expected to be more representative of the 24-hour period. Bartholomew and Hill ${ }^{4}$ showed that urinary nitrate reaches its maximum 4-6 hours after an oral nitrate load, and retums to the baseline value within 18 hours. This suggests that overnight specimens may be useful, in the case of nitrate, also considering the usual consumption time of foods rich in nitrate. In our analysis, we computed the excretion rate per hour and used this as our criterion variable to compare with intake.

Few estimates have been made of the nitrate intake of individuals. The estimates so far have generally been based on population averages. White ${ }^{18}$ estimated daily nitrate intake as $1.19 \mathrm{mmol} /$ day in the US; per capita intakes; for European countries vary mostly between 1.11 and $2.51 \mathrm{mmol} /$ day, ${ }^{25}$ while Japanese per capita estimates amount to $4.52 \mathrm{mmol} / \mathrm{day} .{ }^{20}$ In two recent British studies individual nitrate intake was estimated

TABLE 3 Pearson correlanion coefficients berween urinary nitrate excrenon levels and potential predictors in 59 men and women, Boston. t984

\begin{tabular}{lrr}
\hline & $\begin{array}{c}\text { Nitrate excretion, } \\
\text { mmolhr (In) }\end{array}$ & p-value \\
\hline Gender $(\mathrm{M}=1, \mathrm{~F}=2)$ & -0.48 & $<0.001$ \\
Age, yrs $(\mathrm{In}):$ & 0.32 & 0.013 \\
Queielet's Index, $\mathrm{kg} \mathrm{m}^{2}$ & 0.37 & 0.004 \\
Calories. keal $(\mathrm{In})$ & 0.21 & 0.110 \\
\hline
\end{tabular}


TAate 4 Partial correlation coefficients benwern intake and excreion of nitrate after adjustment for different covariates amone 59 men and wamen, Baston, / 984

\begin{tabular}{lcc}
\hline Adjusting for & $\begin{array}{c}\text { Partial } r \text { (nitrate intake } \\
\text { versus excretion) }\end{array}$ & p-value \\
\hline Nothing (simple $t$ ) & 0.20 & 0.132 \\
Gender & 0.38 & 0.003 \\
Age (In) & 0.16 & 0.230 \\
Ouctelet's Index & 0.25 & 0.058 \\
\hline
\end{tabular}

by a food frequency questionnaire ${ }^{14}$ and a diet record. ${ }^{27}$ Forman et $a l^{1 / 4}$ estimated daily nitrate intake to be $1.89 \mathrm{mmol}$ in areas at low risk for gastric cancer and $1.19 \mathrm{mmol}$ in high risk areas. Using 48-hour dietary records. Chilvers $\mathrm{et} \mathrm{al}^{{ }^{7}}$ estimated this intake to be 1.78 \pm 1.25 mmol among 404 adults ( 177 men, 227 women). No differences according to gender were reported. W/e noted lower intakes in males, although this difference was not significant. Chilverset al, also measured urinary nitrate output and found mean 24-hour excretion levels of $1.94 \pm 1.21 \mathrm{mmol}$, which was slightly higher than their intake (one urine specimen was collected per subject). In our study using two urine collections of approximately 13 hours we estimated excretion rates to be $0.08( \pm 0.04) \mathrm{mmol} / \mathrm{hr}$ in men and 0.05 ( \pm $0.02) \mathrm{mmol} / \mathrm{hr}$ in women, respectively. If our overnight excretion rates would be representative for the daily output, our corresponding 24-hour excretion levels would be $1.90 \pm 1.06 \mathrm{mmol}$ for men and $1.08 \pm$ $0.59 \mathrm{mmol}$ for women.

The percentage of ingested nitrate that was excreted into the urine estimated by our methods differed substantially between males and females. The reasons for this are not clear, but include the possibilities of differential reporting of intake by gender, and chance, particularly since the questionnaire asked about usual intake of foods over the past year and the urines represented only two days. While differences in metabolism of nitrate between men and women cannot be excluded, we have little reason to suspect they might exist. It is also possible, of course, that the questionnaire underestimates intake for males. However, in earlier valida. tion studies on beta-carotene intake $e^{3,2}$ the same questionnaire was able to discriminate between men and women adequately. In that study women were shown to have both higher plasma levels and higher dietary intake of carotene than men.

Overall, assuming that our urine measurements reflected the entire 24-hour intake we found the proportion of ingested nitrate that was excreted to be $77 \%$ in men and women combined. Excreted nitrate represents the combination of exogenous intake and endogenous synthesis corrected for metabolic losses. ${ }^{192}$ The apparent recovery from urine of ingested nitrate will be influ: enced by the relative proportion of these two inputs. Assuming that endogenous synthesis is relatively constant for an individual, higher ingested amounts of nitrate will lead to lower apparent recovery in unne it the endogenous component is not accounted in this balance. ${ }^{19.20 .30}$ The $77 \%$ recovery, however, is in agreement with the estimates made for recovery of ! $\mathrm{NO}_{3}$. where approximately half the metabolic losses appear to be due to the action of the gastrointestinal flora." Despite the substantial variation in nitrate excretion within a person, a reasonabie correlation between intake and excretion was observed after adjustment for gender and Quetelet's Index.

Caloric intake was not correlated with nitrate excretion rate. This might be expected since foods rich in nitrate generally contain few calories (eg vegetables). The absence of any age effect on excretion was expected since the population was relatively homogeneous with respect to age. This group of public health students was also unusual with regard to the Quetelet's Index: males had a higher index than females, contrary to what is generally found. Howevei, no one was grossly obese. This had no adverse effects on the study, since the effect

TABL. 5 Predictors of nutrate excretion (mmollhr), logarithmically transformed, in 59 men and women living in Boston during I984

\begin{tabular}{|c|c|c|c|c|c|c|}
\hline & \multicolumn{3}{|c|}{ Univariate regression } & \multicolumn{3}{|c|}{ Multiple tegressuon. } \\
\hline & poef! & (SE) & $\mathrm{p}$ & coef & (SE). & p \\
\hline Intercept & & & & -5.46 & & \\
\hline Nitrate intake (In mmol/day) & 018 & $(0.13)$ & 0.149 & 0,31 & $(0.11)$ & 0.006 \\
\hline Gender $(M=1, F=2)$ & -0.59 & $(0.15)$ & $<0.00 !$ & -0.58 & $(0.14)$ & $<0.001$ \\
\hline Quetelet's Index $\left(\mathrm{kg} / \mathrm{m}^{2}\right)$ & 011 & (0.04) & 0.006 & 007 & (0.03) & 0.035 \\
\hline Age (In years) & 116 & $(0.47)$ & 0.017 & 0.49 & $(0.41)$ & 0.240 \\
\hline \multicolumn{4}{|l|}{$\mathrm{R}^{2}$} & 0.42 & & \\
\hline \multicolumn{4}{|c|}{ Partial t (nitrate intake vs excretion) } & 037 & & 0.005 \\
\hline
\end{tabular}


of these variables could be controlled for in multiple regression analyses.

In conclusion, our data suggest that a simple selfadministered questionnaire may provide useful information on usual nitrate intake. However, these findings should be replicated among larger and more diverse populations, since the performance of the questionnaire and the between-person variation in dietary sources of nitrate may be different in other demographic groups. The performance may also be different when subjects are living in areas where the nitrate content of drinking water is elevated: questions on water consumption will have to be added in that case.

\section{ACKNOWLEDGEMENTS}

Piet A van den Brandt was supported by a research fellowship from the Netherlands Cancer Foundation (Amsterdam). This investigation was supported by a grant from the National Instatutes of Health. PHS Grant Number 1-P01-CA26731-06, awarded by the National Cancer Institute, DHHS, and by a grant from the American Cancer Society. The authors thank Laura Sampson, RD, who assembled the necessary food composition database and Susan Newman who assisted in the preparation of the manuscript.

\section{REFERENCES}

Willett W C. Sampson L. Stampier M J et al. Reproducibility and validity of a semrquantitative food frequency questionnaire. Am J Epidemiol 1985; 122: 51-65

${ }^{2}$ Beaton $\mathrm{G} \mathrm{H}$. Milner J, Corey P et al. Sources of variance in 24-hour dietary recall data: amplicatons for nutrition study design and inerpretation. Am f Clin Nurr 1979: 32: 2546-59.

'Willett WC. Srampier M J. Underwood B A et al Validation of a dietary quesuonsaise with plasma carotenoid and alphatocopheroi levels. Am I. Clin Nur 1983; 38:63! -9

'Bartholomew. B. Hill M J. The pharmacology of dietary mitrate and the origin of urinary nitrate. Food Chem Foxicol 1984; 22: 789 os.

'Joossens J V. Geboers J. Nutrition and gastre cancer. Nutr Cancer $1981,2: 250-61$.

"National Academy of Sciences. The hratih effecrs of nitrate, nitrite and N.nirnoso compounds. Pari / Washington DC. National Academy Press. 1981

7 Correa P. Haenszel W, Cuello C. Tannenbaum S. R. Archer M. A model for gastric cancer cpudemiology Lancet $1975,2: 58.60$

"Mirvish SS. The efiology of gastne cancer Intragastric nitrosamide formation and other theories. J NCl 1983:71: 629-47

v Tannenbaum SR. Weisman M. Fett D. The effect of nutrate intake on nutrte formation in human saliva. Foud Cosmet Toxicod 1976. 14: 549

"Bos P M J, Van den Brandi P A. Wedel M. Ockhuizen T The reproducibility of the conversion of nitrate to nitrie in human saliva after a nutrate load food Chem Tosucol IUS8, 26: 93-7

"Sander J. Schweinsberg F. LaBar J ef al. Nitnte and nutrosable amino compounds in carcinogencass Giann Monogr 1975, 17; 145-ed]

it Oshuma H. Bartsch H Quantitatre estumaton of endogenous nutro. sation in humans by monitoring $\mathrm{N}$-mutrosoproline excreted in the urine Cancer Res 1981, 41: 3658 .

"Armio R, Gonzalez A. Orellana M. Coubon A H. Sayre J W. Detels R. Epidemiology of gastric cancer in Chile: II. Nitrate exposures and stomach cancer trequency. Imt J Epidemiol 1981. 10: $57-62$

WForman D. Al-Dabbagh S. Doll R. Nitrates, nutrites and gastric cancer in Great Britan. Nature 1985, 313: 620-5.

it Risch H A, Jain M. Chou N W et al. Dietary factors and the incidence of cancer of the stomach. Am J Epidemiol 1985; 122: 947-59

is LuSH. Ohahima H. Fu H Meral Urinary excretion of N-nitrosamino acnds and nitrate by inhabutants of hugh- and low-risk arcas for csophageal cancer in Northern China: endogenous formation of nitrosoproline and its inhibution by vitamin $\mathrm{C}$ Cancer Res $1986 ; 46: 1485-91$

" Berestord S A A. Is nitrate in the drinking water associated with the risk of cancer in the urban UK? Int J Epidemiol 1985; 14 : 57-63.

it Whate I W Relative sugnificance of dietary sources of mutrate and nutrite. S Agrie Food Chem 1975, 23: 886-91

"Green L. C, Ruiz de Luzunaaga K. Wagner D A et al. Nitrate biosynthesus in man. Proc Natl Acad Sci USA 1981: 78: 7764-8.

a Dixon W I (ed) BM(DP Stansincal sofmare. Berkeiey, C A. Unv of Califortua Press. 1983

"Liu K. Stamlet J. Dyer A. Mekéever P. McKeever I Statustical methods to assess and minimize the role of intraindividual variabality in obscuring the relationship between dietary lipids and serum cholesteroli, J Chron Dis 1978: 31 : 390 - 18.

2 Rusner B. Willett W C. Interval estumates for cortelation coefficients corrected for within-person variation: implications for study design and hypothesis testing. Am J Epidemiol 1988; 127: $377-$ 86 .

3 Liu K, Dyer A R. Cooper R. S et al. Can overnight urine seplace 24-hour urine collection to assess salt intake? Hypertension 1979: 1: 529-36

Watson R L. Langiord HG. Usefulness of overnight urines in population groups Pilot studies of sodium. potassium and calcium excretion. Am J Clin Nutr 1970: 23: 290-304

3 Hartman PE. Putative mutagensicarcinogens in foods. I. Nitrate nitrite ingestion and gastric cancer mortality. Environ Mutagen 1983: $5: 111-21$.

s Kawabata. T. Ohshima H. Uibu $J \mathrm{et}$ al. Occurrence. formation and precursors of N-nutroso compounds in Japanese diet. Proceed. ings of the nunth international symposium of the Princess Tak. amatsu Cancer Research Fund. Tokyo. Japan: Univ Tokyo Press 1979: 195-209

"Chilvers $\mathrm{C}$. Inskip H. Caygll $\mathrm{C}$ ct al A survey of dietary nutrate in well-water users. Int J Epidemiol 1984, 13: 324-31

s Stryker W S. Stein E A. Kaplan L et al. The relationships of diet. cigarette smoking. and alcohol consumption with plasma levels of betacarotene and alpha tocopherol. Am f Epidemuol 1988. 127: 283-96

* Wagner D A. Young V R. Tannenbaum S R. Marmmalian nitrate biosynthess: Incorporation of $15 \mathrm{NH}_{1}$ into nutrate is enhanced by endotoxin treatment. Proc Nat Acad Sct USA 1983; 80: 4518 21.

"Lee K. Greget J L. Consaul J R et al. Nitrate, nitrite balance and de novo svnthess of nitrate in humans consuming cured meats. Am J Clin Nuir 1986, 44: 188-94

"Shultz D S. Deen W M. Karel S F. Wagner D A. Tannenbaum S R. Pharmokinctics of nutrate in humans: role of gastrountestinal absorption and metabolism Carcinogenesis 1985; 6: 847-52

(Revised version received March 1989) 


\title{
Brief Reports
}

\section{Cross-sectional versus Longitudinal Investigations of the Diet-Cancer Relation}

\author{
Piet A. van den. Brandt, ${ }^{\prime}$ R. Alexandra Goldbohm, ${ }^{2}$ A. Jeanne M. van Loon, ' and Frans J. Kok ${ }^{2}$
}

Within a prospective cohort study on diet and cancer, information was collected on cancer prevalence and baseline meat consumption. A nested case-control study on meat and cancer was conducted with 656 prevalent colorectal cases, 1,894 breast cancer cases, and 4.701 controls. When analyzed cross-sectionally, prevalence odds ratios for eating meat rarely versus regularly were 2.08 for female colorectal and 1.75 for breast cancer. In the longitudinal analysis, cases who started consuming meat rarely after diagnosis were excluded, resulting in odds ratios of 0.51 for female colorectal and 1.17 for breast cancer. These opposite findings highlight the problem of cross-sectional designs. (Epidemiology 1990;1:402-404)

Keywords: diet, epidemiologic methods, biometry, questionnaires, colorectal cancer, breast cancer

A prerequisite for etiologic inference is that exposure precedes disease; cross-sectional designs are considered inferior for studying etiology. In observational studies on diet and cancer etiology, exposure may be measured with biological specimens or by dietary assessment. Such studies are presumed to be longitudinal, although sometimes observed exposure levels may have been influenced by (preclinical) disease. Changes in various serum nutrient levels owing to rumot growth have been described $(1-6)$. Case-control studies aimed at dietary intake may also be partly cross-sectional, even though they are conducted with incident cases. Interviewing cases about their prediagnostic diet usually occurs within some months after diagnosis. The recall of past dietary habits is influenced by current habits $(7-10)$; when curtent habits of cancer patients have been influenced by (preclinical) disease, the study may contain a crosssectional element.

Little is known about the magnitude of this problem because the necessary data are often not available. In an extreme approach to this issue, we have investigated how odds ratios might be affected when a true crosssectional study is actually conducted. Our study examined the relation between meat consumption and colo. rectal and breast cancer, which has been reported in various studies $(11-19)$.

\footnotetext{
Department of Epidemiology, University of Limburg. P.O. Box 616. 6200 MD Maastricht. The Netherlands. 'Epudemiology Section, TNO-CIVO Toxicology and Nutrition Institute, Zeist, The Netherlands. Address reprint requests to Piet $A$. van den Brandt.

This work was supported in part by the Dutch Cancer Society.

1990 Epidemiology Resources Inc
}

\section{Methods}

We used an ongoing prospective cohort study on diet and cancer. The cohort comprises 120,852 men and women aged 55-69 years. At baseline (1986), cohort members completed a questionnaire on dietary habits and potential confounders. Analysis will involve a casecohort approach for which purpose a random subcohort ( $n=5,000)$ has been selected. Thus, complete questionnaires. will be processed only for subcohort members and incident cancer cases from the cohort (21). One questionnaire page has, however, been processed for ev. ery cohort member to identify all participants and to measure several key variables important for future analyses. Among other questions, this page contained questions on the lifetime prevalence of cancer (with information on site and year of diagnosis) and the frequency of meat consumption. Subjects who ate little or no meat at baseline (0-1 day/week) were also asked to state the year in which they started this habit. The cohort in. cluded 656 colorectal and 1,894 self-reported prevalent breast cancer patients.

The relation berween meat consumption frequency and the prevalence of both colorectal and breast cancer was analyzed in a nested case-control manner using the prevalent cases of both sites; the control group was formed by the 4,701 subjects without cancer from the mentioned subcohort of 5,000 subjects. First, the "crosssectional" association between meat consumption frequency and cancer prevalence, both at baseline, was determined while controlling for age (5-year groups) in a stratified analysis. This analysis was followed by a "longitudinal" analysis in which the timing of exposure and disease was also taken into account. Analyses were carried out for men and women separately. Because it 
INVESTIGATIONS OF THE DIET-CANCER RELATION

TABLE 1. Prevalence Odds Ratios between Frequency of Meat Consumption and Cancer in Cross-sectional Analyses

\begin{tabular}{|c|c|c|c|c|c|}
\hline Tumor Site & Sex & $\begin{array}{l}\text { Meat Consumption } \\
\text { (daysweek) }\end{array}$ & Cases & Controls. & $\mathrm{OR}_{\mathrm{MH}}(95 \% \mathrm{Cl})^{\circ}$ \\
\hline \multirow[t]{2}{*}{ Colorectal } & m & $\begin{array}{l}0-1 \\
2-4 \\
5-7+\end{array}$ & $\begin{array}{r}12 \\
32 \\
282\end{array}$ & $\begin{array}{r}45 \\
246 \\
1.565\end{array}$ & $\begin{array}{l}1.89(0.98-3.63) \\
0.89(0.61-1.32) \\
1.00\end{array}$ \\
\hline & f & $\begin{array}{l}0-1 \\
2-4 \\
5-7^{+}\end{array}$ & $\begin{array}{r}19 \\
54 \\
248\end{array}$ & $\begin{array}{r}73 \\
380 \\
1.945\end{array}$ & $\begin{array}{l}2.08(1.23-3.52) \\
1.13(0.82-1.55) \\
1.00\end{array}$ \\
\hline Breast & $f$ & $\begin{array}{l}0-1 \\
2-4 \\
5-7^{b}\end{array}$ & $\begin{array}{r}92 \\
364 \\
1,409\end{array}$ & $\begin{array}{r}73 \\
380 \\
1,945\end{array}$ & $\begin{array}{l}1.75(1.27-2.39) \\
1.32(1.13-1.55) \\
1.00\end{array}$ \\
\hline
\end{tabular}

- Adjusted for age.

+ Refetence category.

was known for cohort members who consumed meat less than twice a week in what year they started doing so, we were able to determine for the prevalent cases with this habit whether it indeed had preceded cancer diagnosis. The longitudinal analysis was confined to those cases who already consumed meat rarely before diagnosis. Controls who consumed meat rarely were excluded accordingly when they had started this after the earliest year of cancer diagnosis of the cases in their respective age-sex stratum. It was considered unlikely that cases would start eating meat more often after their diagnosis: therefore, the exposure category 5-7 days/week meat consumption is assumed to be constant over time. Because the stability of the category 2-4 days/week meat consumption is unclear in this respect, it is omitted from the analysis. Confidence intervals for Mantel-Haenszel odds ratios were calculated using the formula of Robins et al (22).

\section{Results}

The cross-sectional analysis, in which timing of exposure and disease was ignored, revealed a positive association between infrequent meat consumption and cancer prevalence in both sexes (Table 1).

Fot the ensuing longitudinal analysis" it was found that only $12 \%$ of the 31 colorectal and $26 \%$ of the 92 breast cancer cases who consumed meat 0-1 days/week. at baseline exhibited similar behavior before diagnosis. Since all these cases were female, we were unable to determine which male controls were to be excluded in the stratified analysis. Consequently, longitudinal odds ratio estimates could not be calculated for men. For women, the longitudinal analysis now shows an inverse association between infrequent meat intake and colon cancer, while there is essentially no association with breast cancer (Table 2).

\section{Discussion}

The results of the cross-sectional and longitudinal analysis are in contrast with each other, especially for colon cancer. The odds ratios from the longitudinal analysis. for female colorectal and breast cancer $(0.51$ and 1.17 . respectively) are within the range of values found in other studies (14,17-19) based on incident cases. To investigate whether dietary habits change because of the presence of cancer and what effect this might have on odds ratio estimates, one would ideally conduct a prospective cohort study with baseline dietary assessment and then interview incident cases again after diagnosis about their current and prediagnostic diets. Our results are limited because we had to use prevalent cases for this analysis.

The use of prevalent cases in a longitudinal analysis is problematic when the exposure also acts as a prognostic factor (23). For breast cancer, a high fat intake was found to be weakly associated with a worse prognosis (24). If, for breast and colorectal cancer, a high meat intake were also related to a declined prognosis, it would imply that prevalent cases eating meat rarely are overrepresented. Under these circumstances, the observed longitudinal odds ratios would be biased towards a posirive association, which cannot explain the low odds ratio for colorectal cancer.

The estimates might also be confounded, for example, by fat intake and reproductive variables: such confounding, however, did not explain the relation in various other studies $(14,17,19)$. This possibility will be investigated in detail in the prospective part of the cohort study that incorporates incident rather than prevalent cases (21). Moreover, the type of meat and portion size will also be considered then, since the complete dietary questionnaire is of a semiquantitative nature.

Because prevalent cases are not ideal for studying the 
VAN DEN BRANDT

TABLE 2. Prevalence Odds Ratios between Frequency of Meat Consumption and Cancer in Longitudinal Analyses

\begin{tabular}{|c|c|c|c|c|c|}
\hline Tumor Site & Sex & $\begin{array}{l}\text { Meat Consumption } \\
\text { (days/week) }\end{array}$ & Cases & Controls & $\mathrm{OR}_{\mathrm{MH}}(95 \% \mathrm{Cl})^{\circ}$ \\
\hline Colorectal & $\mathrm{ft}$ & $\frac{0-1}{5-7 \neq}$ & $\begin{array}{r}3 \\
248\end{array}$ & $\begin{array}{r}49 \\
1,945\end{array}$ & $\begin{array}{l}0.51(0.16-1.63) \\
1.00\end{array}$ \\
\hline Breast & $f$ & $\frac{0-1}{5-7 \ddagger}$ & $\begin{array}{r}19 \\
1,409\end{array}$ & $\begin{array}{r}24 \\
1,945\end{array}$ & $1.17(0.63-2.17)$ \\
\hline
\end{tabular}

- Adjusted for age.

$\uparrow$ No estimates for men available (see text).

t Reference category.

etiologic role of this type of exposure, no great importance should be attached to the absolute values of the odds ratios reported here. Nevertheless, this analysis indicates that, for colon cancer, the direction of the association might be reversed when cross-sectional instead of longitudinal analyses are performed. For breast cancer, the positive association largely disappeared when a longitudinal analysis was done. These data indicate that the presence of cancer may result in a change of dietary habits, and they confirm the limitations of truly crosssectional studies for etiologic inference. As mentioned earlier, data in some studies with a presumably longitu. dinal design may to some extent be cross-sectional. We believe that the quantification of this problem and its implications for bias warrant further study.

\section{References}

1. Wald N, Idle $\mathrm{M}$, Boreham I. Bailey A. Low serum-vitamin-A and subsequent risk of cancer. Lancet 1980;it:813-5.

2. Wald N, Boteham J, Bailey A. Serum retinol and subsequent risik of cancer. Br J Cancer 1986:54:957-61.

3. Wald NJ. Thompson SG, Densem JW, Boreham J, Bailey A Serum vitamin $\mathrm{E}$ and subsequent risk of cancer. $\mathrm{Br}$ J Cancer 1987:56:69-72.

4. Wald NJ. Thompson SG, Densem JW, Boreham J, Bailey A. Serum beta-carotene and subsequent risk of cancer: results from the BUPA Study. Br ! Cancer 1988:57:428-39.

5. Robinson MF, Godfrey PJ. Thomson CD, Rea HM, Van Rij AM. Blood selenium and glutathione peroxidase activity in normal subjects and in surgical patients with and without cancer in New Zealand. Am J Clin Nutr 1979;32:1477-85.

6. McMichael AJ. Jensen OM, Parkin DM. Zaride DG. Dietary and endogenous cholesterol and humart cancer. Epidemiol Rev 1984:6:192-216.

7. Mollet Jensen $\mathrm{O}$, Wahrendorf J, Rosenqvist: $\mathrm{A}$, Geser $\mathrm{A}$. The reliability of questionnaire-derived historical dietary information and temporal stability of food habits in individuals. Am J Epidemiol 1984:120:281-90.

8. Jain M. Howe GR, Harrison L, Miller AB. A study of repeatability of dietary data over a seven-year period. Am J Epidemiol $1989 ; 129,422-9$.
9. Byen TE, Rosenthal RI, Marshall JR, Reepka TF, Cummings KM. Graham S. Dietary history from the distant past: a method. ological study. Nutr Cancet 1983:5:69-77.

10. Thompson FE, Lamphiear DE, Metuner HL, Hawthome VM, Oh MS. Reproducibility of reports of frequency of food use in the Tecumseh Diet Methodology Study. Am / Epidemiol 1987. 125:658-71.

11. Haenasel W. Berg JW. Segi M, Kurihara M, Locke FB, Large bowel cancer in Hawatian lapanese. JNCI 1973:51:1765-79.

12. Manousos O. Day NE, Trichopoulos D. Gerovasuilis F. Tzonou A. Diet and colorectal cancer a case-control study in Greece. Int J Cancer 1983,32:1-5.

13. Milijer AB, Howe GR, Jain M, Craib KJP, Harrison L. Food items and food groups as risk factors in a case-control study of diet and colo-rectal cancer. Int J Cancer 1983:32:155-61.

14. Kune S, Kune GA, Watson LF. Case-control study of dietary etiological facton: the Melbourne colorectal study. Nutr Cancer 1987:9:21-42.

15. L Vecchis C, Negri E, Decarli A, D'A vanzo B, Galloti L, Gentile A. Franceschi S. A case-control study of diet and colo. rectal cancer in northem Italy, Int ] Cancer 1988:41:492-8.

16. Hirayama $T$. Epidemiology of breast cancer with special reference to the role of diet. Prev Med 1978;7:173-95.

17. Lubin $[H$. Burns PE, Blot W], Ziegler RG, Lees AW, Fraumeni JF. Dietary factors and breast cancer risk. Int / Cancer 1981, 28.685-9.

18. Talamini R, La Vecchia C, Decarli A. Franceschi S, Grattoni E. Grigolerto E, Liberati A. Tognoni G. Social facton, diet and breast cancer in a northern Italian population. Br / Cances 1984:49:723-9.

19. La Vecchia C, Decarli A. Franceschi S, Gentile A. Negri E, Parazsini F. Dietary factors and the risk of breast cancer. Nutr Cancer 1987;10:205-14.

20. Rogen $A E$, Longnecker MP. Dietary and numitional influences on cancer: a review of epidemiologic and experimental data. Lab Invest 1988,59.729-59.

21. Van den Brandt PA, Goldbohms RA, Van 't Veer P, Volovica A, Hermus RJj, Sturmans F. A large-scale prospective cohort study on diet and cancer in the Netherlanda. J Clin Epidemiol 1990, 43:285-95.

22. Robins JM. Breslow NE, Greenland S. Estimates of the Mantel. Haenssel variance consistent in both sparse-data and large-strata limiting models. Biometric 1986:42:311-23.

23. Kleinhaum DG, Kupper L., Morgenstern H. Epidemiologic research: principles and quantitative methods. Belmont, CA: Lifetime Learning. 1982

24. Verteault R., Brison J, Deschenes L, Naud F, Meyet F, Belanger L. Dietary fat in relation to progenostic indicaton in breast cancer. JNCI 1988:80:819-25. 



\title{
Chapter 10
}

\section{Stratified and simple regression methods for the analysis of case-cohort studies*}

\author{
Alexander Volovics', Piet A. van den Brandt ${ }^{2}$. \\ 1) Department of Medical Statistics and Informatics, Universiry of Limburg, Maastricht, the Netherlands; 2) \\ Department of Epidemiology, University of Limburg, Maastricht, the Netherlands
}

\begin{abstract}
Case-cohort and nested case-control sampling methods have recently been introduced as a means of reducing cost in large cohort studies. The asymptotic distribution theory results for relative rate estimation based on Cox type partial or pseudollkelihoods for case-cohort and nested case-control studles have been accounted for. However, many researchers use elementary (stratified) methods for a first or primary summarization of the most important evidence on exposure-disease or dose-response relationships, l.e. the classical Mantel-Haenszel analyses, trend tests and tests for heterogenelty of relative rates, which can be followed by exponentlal fallure time regresslon methods on grouped and individual data to model relationships between several factors and response. In this paper we present the adaptations needed to use these methods with case-cohort designs, illustrating their use with data from a recent case-cohort study on the relationship between diet, life-style and cancer.
\end{abstract}




\section{Introduction}

The germs of the ideas of nested case-control and case-cohort sampling from a cohort can be found scattered through the statistical and epidemiological literature of the 60 's and 70 's. In the failure time context Thomas (1) appears to have first comprebensively formulated the nested case-control approach while Prentice (2) proposed the casecohort design as a more efficient solution, in some situations, to sampling cohort subjects than the nested case-control sampling design.

The general approach to nested case-control sampling involves the selection of a random sample without replacement of subjects at risk but without disease (control subjects) at each distinct failure time (every time a case is observed). Selected controls remain eligible for control selection at other failure times. The control selection procedures at distinct failure times are statistically independent. Relative rate estimation can be based on a Cox type partial likelihood approach. Curiously this approach has only recently been theoretically justified by Goldstein and Langholz (3). When studying a range of disease endpoints this design can pose logistical and administrative problems as a different random sample of controls has to be selected each time a specific endpoint is observed. Furthermore, collecting and processing of covariate information on controls can only start at the time of the first failure and all covariate information will only be available at the end of the study period.

The case-cohort design avoids these problems by selecting a subcohort randomly from the entire cohort which then provides a comparison group at each disease occurrence time, i.e. those subjects in the subcohort still at risk for the disease under study at a given failure time function as controls for the occurring failure whether that failure occurs 'inside' or 'outside' the subcohort. This design allows the comparison group to be selected in advance of cohort follow-up, a distinct advantage since the subcohort can then be used, for example, to monitor the achievement of intervention goals and the collection and processing of covariate information for all controls (the subcohort members) can be started immediately on inception.

Also, in contrast to the control sample in the time-matched nested case-control design, the subcohort provides a natural comparison group for a range of disease endpoints. Prentice (4) provided heuristic justification for relative rate regression analysis based on a pseudolikelihood approach. Full theoretical justification for the proposed methods was presented by Self and Prentice (5). Readers interested in further methodological details regarding the above and other similar designs can consult various references $(2,4-11)$.

We will concentrate here on procedures for the analyses of case-cohort studies. The organization of this paper is as follows: $\$ 2$ presents a heuristic introduction, $\$ 3$ presents necessary preliminary calculations and notation, $\$ 4$ contains the adaptations for the elementary (stratified) methods, $\$ 5$ contains the adaptations for exponential failure time regression, $\$ 6$ contains an illustration of the procedures in a case-cohort analysis of smoking and lung cancer. In $\$ 7$ we discuss the procedures and the two approaches: 'blow-up' and 'shrink'. All formulas that will be presented were programmed in GLIMcode; these macros are available upon request. 


\section{Heuristic introduction to case-cohort analyses}

The elementary analysis of cohort studies and Poisson regression for grouped data are comprehensively described in Breslow and Day (12), chapters 2, 3, 4 and 5, to which we refer for standard formulas and practice. 'Poisson' regression for individual data is described in Aitkin et al. (13), chapter 6.

The results of a cohort and case-cohort study with only two exposure groups can be summarized as in table 1 , $a$ and $b$.

Table 1. Summarized results of a cohort and case-cohort study involving a dichotomous exposure.

a. Cohori study

b. Case-Cohori

Exposure Observed Person years

failures
Observed Person years

failures

Failures Censored Total Failures Censored Total

$\begin{array}{lllllllll}1 & d_{1} & t_{d 1} & t_{c 1} & t_{1} & d_{1} & t_{d 1} & t_{k 1} & t_{1} \\ 2 & d_{2} & t_{d 2} & t_{c 2} & t_{2} & d_{2} & t_{d 2} & t_{k 2} & t_{2}\end{array}$

The notation $t_{d i}, t_{c i}, t_{s e}$ in table 1 indicates person years for failures, for censored individuals and for censored individuals in the subcohort only, respectively. Censoring is due for example to ending of follow-up, withdrawals or competing causes of failure.

Assuming a constant failure rate during the study period the failure rate $\lambda_{i}$ for exposure group $i$ in the cohort study can be estimated as $\lambda_{i}=d_{i} / t_{i}$, the number of observed cases divided by the total person years of exposure in group $i, i=1,2$. The relative rate of exposure 2 versus exposure 1 can be estimated as $\hat{i}_{i}=\hat{\lambda}_{2} / \hat{\lambda}_{1}=d_{2} t_{1} / d_{1} t_{2}$. It will be intuitively clear that the analogous statistic $d_{2} t_{1} / d_{1} t_{2}$ in the case-cohort set-up should in general not be used to estimate the relative rate. The person years of the failures is the same in both studies but the person years of the censored individuals in the case-cohort study is a random sample from the censoring times in the cohort study. If the sampling. fraction is $\rho=m / n$ with $m$ denoting subcohort and $n$ cohort size, we can expect that $t_{c i} \approx \rho t_{c i}$. Thus $d_{2} z_{1} / d_{1} z_{2} \approx d_{2}\left(t_{d 1}+\rho t_{c 1}\right) / d_{1}\left(t_{d 2}+\rho t_{c 2}\right)$ and this will in general not be close to $d_{2} t_{1} / d_{1} t_{2}$.

Two solutions present themselves:

a. 'Blow-up' the person years of the censored individuals in the case-cohort study by a factor $\rho^{-1}$, that is to say use $t_{d i}+\rho^{-1} t_{c i} \approx t_{i}$ instead of $t_{i}$.

Using the 'blow-up' method we estimate $\downarrow$ by

$\psi^{B}=d_{2}\left(t_{d 1}+p^{-1} t_{s c 1}\right) / d_{1}\left(t_{d 2}+\rho^{-1} t_{s c 2}\right) \approx \downarrow$.

b. 'Shrink' the person years of the failures by a factor $\rho$, i.e. use

$\rho t_{d i}+t_{\text {sci }}$ instead of $\tilde{t}_{\mathrm{r}}$. The same effiect could of course be accomplished by using only the total person years of subcohort members, namely $t_{\mathrm{bi}}=\mathrm{t}_{\mathrm{dil}}+\mathrm{t}_{\mathrm{rd}}$ where $\mathrm{t}_{\mathrm{bdi}}$ is the observed person years for failures in the subcohort only: $t_{i} \approx \rho t_{i}$. Using the 'shrink' method we estimate $\downarrow$ by $\psi^{s}=d_{2} t_{s 1} / d_{1} t_{s 2} \approx d_{2} t_{1} / d_{1} t_{2}=\hat{\phi}$. 
It is important to note that the 'blow-up' method gives direct estimates of the exposure specific failure rates, namely $\hat{\lambda}_{i}{ }^{B}=d_{i} /\left(t_{d_{i}}+\rho^{-1} t_{s c i}\right) \approx \hat{\lambda}_{i n}$. With the 'shrink' method we cannot use the analogous estimator for the exposure specific rates because

$d_{i} / t_{i i} \approx \rho^{-1} d_{i} / t_{i}=\rho^{-1} \lambda_{i}$. The obvious corrected estimator for $\lambda_{i}$ is $\rho d_{i} / t_{i i}$. We shall also say that the 'shrink' method estimates blown-up exposure specific rates $\mu_{i}=\rho^{-1} \lambda_{i}$ with estimators $\hat{\mu}_{i}=d_{i} / t_{i}$; to estimate the $\lambda_{i}$ we have to 'shrink' $\hat{\mu}_{i}$ by the sampling fraction: $\dot{\lambda}_{i}=\rho \hat{\mu}_{i}$.

Confidence intervals for $\hat{\psi}$ are usually calculated using estimated asymptotic variances of $\log$ \&. Concentrating for the moment on the 'shrink' approach we have:

$$
\begin{aligned}
\log \psi^{s} & =\log \left\{d_{2} t_{21} / d_{1} t_{22}\right\} \\
& =\log \left\{d_{2} t_{1} / d_{1} t_{2}\right\}+\left\{\log \left(t_{2} / n\right)-\log \left(t_{22} / m\right)\right\}-\left\{\log \left(t_{1} / n\right)-\log \left(t_{31} / m\right)\right\} \\
& =\log \psi+D^{s}, \text { say. }
\end{aligned}
$$

This equality illustrates 2 things.

Firstly, if a suitably normalized version of $D^{5}$ converges in distribution to a nondegenerate random variable, then as was to be expected, $\hat{\psi}^{\mathrm{s}}$ will be an inefficient estimate of $\psi$ compared to $\hat{\psi}$, having a larger asymptotic variance (AV): namely $\mathrm{AV}\left(\log \hat{\psi}^{5}\right)=\mathrm{AV}(\log \hat{\psi})+\mathrm{AV}\left(\mathrm{D}^{5}\right)$. (It can be shown that $\hat{\psi}$ and $\mathrm{D}^{5}$ are asymptotically independent).

Secondly, given the $n$ individual failure times constituting the $t_{i}, i=1,2$, the variance of $\mathrm{D}^{s}$ is due to random sampling of $\mathrm{m}$ failure times from the finite population of cohort failure times and in estimating the variance of $\log \hat{4}^{5}$ this extra finite sampling variability will have to be accounted for. This means that "naive" estimators for $\mathrm{AV}\left(\log \hat{\psi}^{\mathrm{s}}\right)$ calculated analogously to the estimator for $\mathrm{AV}(\log \hat{\boldsymbol{\psi}})$ will underestimate the true $\mathrm{AV}$ and should not be used. Analogous reasoning can be used to show comparable results for $\hat{\psi}^{\mathrm{B}}$.

In the classical situation where a Poisson distribution is assumed for the number of failures in each cell of a table determined by stratum and exposure group combinations or an exponential distribution is assumed for the individual failure times (see Breslow and Day (12)), it can be shown that the Mantel-Haenszel test, the test for trend and the test for heterogeneity of relative risk are asymptotic approximations of finite sample uniformly most powerful unbiased tests. The Mantel-Haenszel estimator (which can be derived as a weighted combination of stratum specific relative risks, as the first iteration of the maximum likelihood estimator or via an estimating function approach), is a consistent estimator of the relative rate. Even though it is not generally mentioned, the classical set up can accommodate multiple "failures" per individual. If it is suspected that the failure rate does not remain constant during the study period, or to accommodate time dependent exposure, the time axis can also be stratified, assuming a constant failure rate on each stratum. In the classical set up censoring not due to ending of follow-up must, just as failure, be a very rare phenomenon to justify the Poisson assumption.

In adapting the simple methods for case-cohort sampling we will at the same time assume a more general set up than in the classical situation, namely: a piecewise constant failure rate, possible recurrent events (multiple 'failures') per individual, independent censoring and possible left-truncation. The assumption of independent censoring roughly means that at any time the survival experience in the future is not statistically aitered (from what it would have been without censoring), by censoring and survival experience in the past. This is the most general assumption possible with respect to censoring (14). Left truncation, in the simple form of only including subjects 
conditionally on being alive (not having failed) at a certain given calendar time or age, is quite common to cohort studies. These assumptions should guarantee applicability of the methods and reliability of the analysis results for most types of cohort studies whatever being the dynamics driving the process (subject of course to the accuracy of the asymptotic approximations). Under these conditions the adapted Mantel-Haenszel estimator remains a consistent estimator. However the tests have only 'large sample', i.e. asymptotic optimality. Full details concerning all results are presented by Volovics (15).

\section{Organization of the data}

In the classical set up, calculation of the above-mentioned elementary statistics and regression analyses are based on a tabulation of observed failures $d_{j k}$ and person years $t_{j k}$ by stratum $(j=1,2, \ldots, J)$ and exposure group $(k=1,2, \ldots, K)$ (e.g. table 3.4 in Breslow and Day (12)). The $\mathbf{J}$ strata might be determined by one factor such as age or by an amalgamation of two or more variables such as for example age and sex. The $\mathrm{K}$ exposure classes can also result from the amalgamation of two or more exposure variables.

In the case-cohort set up a similar table can be made substituting for cohort person years $t_{j k}$ either 'blow-up' person years $t_{d j k}+\rho^{-1} t_{\text {scik }}$ or 'shrink' (subcohort) person years $t_{\mathrm{jjk}}=\mathrm{t}_{\mathrm{sdjk}}+\mathrm{t}_{\mathrm{cjk}}$, both of which we shall simply denote by $\mathrm{t}_{\mathrm{jk}}$ too. Such a table contains sufficient information to calculate the Mantel-Haenszel estimator and the chi-square heterogeneity tests. To calculate standard errors, the Mantel-Haenszel test and the trend tests, however, some further quantities are needed, namely a stratum-exposure specific (cell-specific) variance for which we have two possible estimates: $v_{j \mathrm{k}}$ or $\mathrm{v} \mathrm{j}_{\mathrm{j} \text {. }}$ Given cell-specific rates, $\theta_{j k}$ say, $v 1_{j k}$ (or $v 2_{j k}$ ) is an estimate of the asymptotic variance of the cell-specific 'residual': $d_{j k}-a_{j k} t_{j k}$ (observed minus 'expected' failures). The $v 1_{j k}$ (or $v 2_{j \mathrm{k}}$ ) are needed because all statistics discussed in this paper are functions of these cellspecific 'residuals' so that their asymptotic variances are also functions of these cellspecific variances (see appendix for more details). In the classical set-up $v_{j k}=\hat{\theta}_{j k} t_{j v}$ where $\hat{\theta}_{j \mathrm{k}}$ is the maximum likelihood estimate of $\theta_{j \mathrm{k}}$. All these quantities are summarized in table 2 where again, just as with the $t_{j k}$, we have used one symbol $\left(v 1_{j k}, v 2_{i k}\right)$ to denote quantities that are calculated differently depending on whether a 'blow-up' or 'shrink' approach is used. We shall comment on a possible choice in the discussion. $d_{j k}$ always stands for the total number of observed stratum-exposure specific failures, whichever approach is used.

To indicate how the table entries are calculated we present formulas for a basic study design with simultaneous entry of all cohort members at the starting date, a constant hazard rate for the duration of the study period, one type of nonrecurrent failure and no time dependent exposure. Amendations to the formulas on departure from this basic design are essentially self evident; details are presented by Volovics (15). The total number of individuals in the case-cohort study itself is $\tilde{n}=m+$ the (random) number of subjects failing, for the failure type under study, outside the subcohort.

For each individual $i$ in the case-cohort study $u_{i j}, x_{i k}, s_{i}$ and $d_{i}$ are indicator variables defined as follows: $u_{i j}=1$ if subject $i$ is in stratum $j$ and 0 otherwise, $x_{i p}=1$ if subject $i$ is in exposure group $k$ and 0 otherwise, $s_{i}=1$ if subject $i$ has been selected for the subcohort and 0 otherwise and $d_{i}=1$ indicates if subject $i$ has failed while $d_{i}=0$ indicates if subject: i was censored. The reference exposure category will always be 
coded 1 ; thus $x_{i 1}=1$ indicates that subject $i$ is in the reference exposure group. For each individual $\mathrm{i}$ in the case-cohort study $\mathrm{t}_{\mathrm{i}}$ is the person years of observation.

The classical elementary methods all assume an underlying relative or multiplicative rate model, i.e. $\theta_{j k}=\lambda_{j} \Psi_{k}$, where $\lambda_{j}=\theta_{j 1}\left(\downarrow_{1}=1\right)$ is the rate for individuals in the baseline or nonexposed category $(k=1)$ in stratum $\mathbf{j}$. $\hat{\psi}_{\mathbf{k}}$ will denote a Mantel-Haenszel or maximum likelihood estimate of the relative rate of failing in exposure group $\mathbf{k}$ as against failing in the reference exposure group 1 ; thus $\hat{\psi}_{1}=1$ always. $\hat{\lambda}_{j}\left(=\partial_{j 1}\right)$ denotes a maximum likelihood estimate of the stratum $\mathrm{j}$ specific failure rate for the reference exposure category. $B$ or $S$ attached to the formula number will indicate whether the 'blow-up" or 'shrink' version is being presented. The cell-specific number of failures $\left(d_{j k}\right)$, person years $\left(t_{j k}\right)$ and variance estimates $\left(v 1_{j k}\right.$ and $\left.v 2_{j k}\right)$ are calculated as follows:

Table 2. Summarization by stratum and exposure level of basic quantities needed for the elementary statistical analysis of case-cohori studies assuming either a 'blow-up' or 'shrink' approach.

\begin{tabular}{|c|c|c|c|c|c|}
\hline Stratum & Exposure & Failures & Person years & Variance1 & Variance2 \\
\hline 1 & $\begin{array}{l}1 \\
2 \\
\dot{K} \\
\dot{K}\end{array}$ & $\begin{array}{l}d_{11} \\
d_{12} \\
\vdots \\
d_{1 K}\end{array}$ & $\begin{array}{l}t_{11} \\
t_{12} \\
\dot{0} \\
\dot{t_{1 K}}\end{array}$ & $\begin{array}{l}v 1_{11} \\
v 1_{12} \\
\dot{\bullet} \\
\dot{v} 1_{1 K}\end{array}$ & $\begin{array}{l}v 2_{11} \\
v 2_{12} \\
\dot{v} \\
\dot{v} 2_{1 K}\end{array}$ \\
\hline . & $\begin{array}{l}1 \\
2 \\
: \\
\dot{K}\end{array}$ & $\begin{array}{l}\mathrm{d}_{21} \\
\mathrm{~d}_{22} \\
\dot{d_{2 k}}\end{array}$ & $\begin{array}{l}\mathrm{t}_{21} \\
\mathrm{t}_{22} \\
\dot{\mathrm{t}_{22}}\end{array}$ & $\begin{array}{l}v 1_{21} \\
v 1_{22} \\
\dot{-} \\
\dot{v} 1_{2 K}\end{array}$ & $\begin{array}{l}v_{21} \\
v_{22} \\
\dot{-} \\
\dot{v} 2_{2 k}\end{array}$ \\
\hline : & : & : & : & : & $\dot{\bullet}$ \\
\hline J & $\begin{array}{l}1 \\
2 \\
\vdots \\
\dot{k}\end{array}$ & $\begin{array}{l}\mathbf{d}_{11} \\
d_{J_{2}} \\
\dot{d_{1 K}}\end{array}$ & $\begin{array}{l}\mathrm{t}_{11} \\
\mathrm{t}_{\mathrm{n}} \\
\dot{\mathrm{t}_{\mathrm{JX}}}\end{array}$ & $\begin{array}{l}v 1_{J_{1}} \\
v 1_{J_{2}} \\
\dot{v} \\
\dot{v} 1_{J K}\end{array}$ & $\begin{array}{l}v_{\mathrm{J}_{1}} \\
\mathrm{v}_{\mathrm{J} 2} \\
\dot{v_{\mathrm{JK}}}\end{array}$ \\
\hline
\end{tabular}

$$
d_{j k}=\sum_{i=1}^{n} u_{1 j} x_{1 k} d_{1}
$$

$$
t_{j k}=\sum_{i=1}^{n} u_{i j} x_{i k}\left[d_{i}+p^{-1}\left(1-d_{i}\right) s_{i}\right] t_{i}
$$




$$
t_{j k}=\sum_{i=1}^{n} u_{i j} x_{i k} s_{i} t_{i}
$$

$$
v 1_{j k}=\sum_{j=1}^{n} u_{1 j} x_{1 k}\left[d_{1}-t_{k} \lambda_{j}\left[d_{1}+p^{-1}\left(1-d_{1}\right) s_{1}\right] t_{1}\right]^{2}
$$

$$
v 1_{j k}=\sum_{i=1}^{n} u_{i j} x_{1 k}\left[d_{1}-\varphi_{k} \lambda_{j} s_{1} t_{1}\right]^{2}
$$

$$
v 2_{j k}=\varphi_{k} \lambda_{j} \sum_{i=1}^{n} u_{i j} x_{1 k}\left[d_{1}+\rho^{-1}\left(1-d_{1}\right) s_{1}\right] t_{i}+
$$

$$
(1-\rho)\left(\varphi_{k} \lambda_{j}\right)^{2} \sum_{i=1}^{n} u_{i j} x_{1 k}\left(1-d_{1}\right) s_{1} \rho^{-2} t_{1}^{2}
$$

$$
v 2_{j k}=\varphi_{k} \lambda_{j} \sum_{i=1}^{n} u_{i j} x_{1 k} s_{i} t_{i}+(1-\rho)\left(\varphi_{k} \lambda_{j}\right)^{2} \sum_{i=1}^{n} u_{1 j} x_{1 k} s_{1} t_{1}^{2}
$$

The estimates $\hat{\psi}_{k}$ and $\hat{\lambda}_{j}$ in formulas $3.3 \mathrm{~B}, 3.3 \mathrm{~S}, 3.4 \mathrm{~B}$ and $3.4 \mathrm{~S}$ will be specified below when needed. In the following 2 paragraphs all formulas are expressed in terms of the quantities $d_{j k}, t_{j k}$ and $v_{j k}$ where $v_{j k}$ stands for either $v 1_{j k}$ or $v 2_{j k}$ depending on which cellspecific variance estimate is used. For the 'blow-up' and 'shrink' approach the quantities 3.2 B, $3.3 \mathrm{~B}$ (or $3.4 \mathrm{~B}$ ) and $3.2 \mathrm{~S}, 3.3 \mathrm{~S}$ (or $3.4 \mathrm{~S}$ ) are to be used, respectively.

\section{Case-cohort adaptations for elementary (stratified) methods}

\subsection{The Mantel-Haenszel estimator}

Given table 2 and a fixed exposure group $k$ the Mantel-Haenszel estimator ${ }_{k}$ for the relative rate $\psi_{k}$ of failure in exposure group $k$ with respect to failure in the reference exposure group is just the well known formula:

$$
\varphi_{k}=\left[\sum_{j=1}^{J} d_{j k} t_{j 1} /\left(t_{j 1}+t_{j k}\right)\right] /\left[\sum_{j=1}^{J} d_{j 1} t_{j k} /\left(t_{j 1}+t_{j k}\right)\right]
$$

with relevant quantities from table 2 plugged in. The asymptotic variance of the logarithm of $\hat{\Psi}_{k}$ can be estimated by

$$
v_{k}=\frac{\sum_{j=1}^{J}\left[\frac{\oplus_{k}^{2} t_{j k}^{2} v_{j 1}+t_{j 1}^{2} v_{j k}}{\left(t_{j 1}+t_{j k}\right)^{2}}\right]}{\oplus_{k}^{2}\left[\sum_{j=1}^{J}\left(\frac{t_{j 1} t_{j k}}{t_{j 1}+t_{j k}}\right) \lambda_{j}\right]^{2}}
$$


where $\hat{\psi}_{k}$ is the above Mantel-Haenszel statistic, $\dot{\lambda}_{j}=\left(d_{j 1}+d_{j k}\right) /\left(t_{j 1}+\hat{\psi}_{k} t_{j k}\right)$ and naturally these same $\hat{\psi}_{k}$ and $\dot{\lambda}_{j}$ are used to calculate $v_{j 1}$ and $v_{j k}$. If we plug in the classical estimates $v_{j 1}=\hat{\lambda}_{j} t_{j 1}$ and $v_{j k}=\hat{\psi}_{k} \hat{\lambda}_{j} t_{j k}$ then formula 4.2 reduces to the classical estimate of $\mathrm{AV}\left(\log \hat{\psi}_{1}\right)$.

If we substitute $v_{j k}$ for the $v_{j b}$ formula 4.2 can be expanded as

$$
v_{k}=\frac{\sum_{j=1}^{J}\left[\frac{t_{j 1} t_{j k}\left(d_{j 1}+d_{j k}\right)}{\left(t_{j 1}+t_{j k}\right)^{2}}\right]}{\varphi_{k}\left[\sum_{j=1}^{J}\left(\frac{t_{j 1} t_{j k}}{t_{j 1}+t_{j k}}\right) \lambda_{j}\right]^{2}}+(1-\rho) \frac{\sum_{j=1}^{J}\left[\frac{\lambda_{j}^{2}\left(t_{j k}^{2} q_{j 1}+t_{j 1}^{2} q_{j k}\right)}{\left(t_{j 1}+t_{j k}\right)^{2}}\right]}{\left[\sum_{j=1}^{J}\left(\frac{t_{j 1} t_{j k}}{t_{j 1}+t_{j k}}\right) \delta_{j}\right]^{2}}
$$

with 'blow-up'

$$
q_{j k}=\sum_{i=1}^{n} u_{1 j} x_{11}\left(1-d_{1}\right) s_{i}\left(\rho^{-1} t_{i}\right)^{2}
$$

or 'shrink'

$$
\mathrm{q}_{j \mathrm{k}}=\sum_{1=1}^{\mathrm{n}} \mathrm{u}_{1 j} \mathrm{x}_{11} \mathrm{~s}_{1} \mathrm{t}_{1}^{2}
$$

The reader will recognize the classical estimator of the asymptotic variance of the logarithm of the Mantel-Haenszel estimator in the first term. We have written this formula as a sum of two terms to illustrate again that plugging the relevant quantities from table 2 into the classical formula would give an underestimate of the asymptotic variance even though the second term will in general be small; see the example in $\$ 6$.

\subsection{The Mantel-Haenszel test}

We will present the Mantel-Haenszel test for the hypothesis $\psi_{k}=1$ for a given exposure category $\mathrm{k}$ with respect to the reference category 1 in the one degree of freedom chisquare form: $\mathrm{NUM}_{\mathrm{k}}^{2} / \mathrm{DEN}_{\mathrm{k}}$ with

$$
\begin{gathered}
\operatorname{NUM}_{k}=\sum_{j=1}^{J}\left(d_{j k}-\AA_{j} t_{j k}\right) \\
\operatorname{DEN}_{k}=\sum_{j=1}^{J}\left[\left(\frac{t_{j k}}{t_{j 1}+t_{j k}}\right)^{2} v_{j 1}+\left(\frac{t_{j 1}}{t_{j 1}+t_{j k}}\right)^{2} v_{j k}\right]
\end{gathered}
$$

Use $\dot{\lambda}_{j}=\left(d_{j 1}+d_{j k}\right) /\left(t_{j 1}+t_{j k}\right)$ and $\dot{\psi}_{k}=1$ to calculate 4.3 and $v_{j 1}$ and $v_{j k}$ in (4.4). If we plug in the classical estimates for $\mathrm{v}_{\mathrm{j} 1}$ and $\mathrm{v} 2_{\mathrm{j}}$ then $(4.4)$ reduces to the classical variance estimator.

\subsection{Trend tests}

Trend tests are usually presented in their one degree of freedom chi-square form, namely: $\mathrm{NUM}^{2} / \mathrm{DEN}$. For the test for trend in the stratum specific rate ratios given a certain exposure category $\mathrm{k}$ we have: 


$$
N U M=\sum_{j=1}^{J} z_{j}\left(d_{j k}-\varphi_{k} \lambda_{j} t_{j k}\right)
$$

and

$$
\mathrm{DEN}=\sum_{j=1}^{J} z_{j}^{2}\left(\frac{\mathrm{v}_{j 1} \mathrm{v}_{j \mathrm{k}}}{\mathrm{v}_{j 1}+\mathrm{v}_{j k}}\right)-\left(\sum_{j=1}^{J} z_{j}\left(\frac{\mathrm{v}_{j 1} \mathrm{v}_{j k}}{\mathrm{v}_{j 1}+\mathrm{v}_{j k}}\right)\right)^{2} /\left(\sum_{j=1}^{J}\left(\frac{\mathrm{v}_{j 1} \mathrm{v}_{j k}}{\mathrm{v}_{j 1}+\mathrm{v}_{j k}}\right)\right)
$$

where the $\mathrm{z}_{\mathrm{i}}$ are quantitative variables representing the level of a stratum defining variable, for example the age level in age stratum $\mathrm{j}$. Here $\dot{\lambda}_{j}$ and $\hat{\phi}_{\mathrm{k}}$ are maximum likelihood estimators under the null hypothesis of no trend: $\hat{\lambda}_{j}=\left(d_{j 1}+d_{j k}\right) /\left(t_{j 1}+\hat{\psi}_{L} t_{j k}\right)$ and $\hat{\psi}_{\mathrm{k}}$ can be obtained as the iterative solution to the equation:

$$
\Psi_{k}=\left[\sum_{j=1}^{J}\left(\frac{d_{j k} t_{j 1}}{t_{j 1}+\Psi_{k} t_{j k}}\right)\right] /\left[\sum_{j=1}^{J}\left(\frac{d_{j 1} t_{j k}}{t_{j 1}+\Psi_{k} t_{j k}}\right)\right]
$$

or by fitting a regression model ( $\$ 5)$.

See Breslow and Day (12, pages 110 and 111) on using the Mantel-Haenszel estimator instead of the maximum likelihood estimator. (The Mantel-Haenszel estimator is the first step iteration of the maximun likelihood estimator obtained by substituting 1 for $\psi_{k}$ on the right hand side of the above equation in $\psi_{k}$ ).

The test for trend in relative rates with increasing exposure has:

$$
N U M=\sum_{k=1}^{K} x_{k}\left(\sum_{j=1}^{J} d_{j k}-\sum_{j=1}^{J} \lambda_{j} t_{j k}\right)
$$

and

$$
D E N=\sum_{k=1}^{K} x_{k}^{2}\left(\sum_{j=1}^{J} v_{j k}\right)-\sum_{j=1}^{J}\left[\left(\sum_{k=1}^{K} x_{k} v_{j k}\right)^{2} /\left(\sum_{k=1}^{K} v_{j k}\right)\right]
$$

Here, in (4.7) and the calculation of the $v_{j k}$ in (4.8), we take $\hat{\psi}_{k}=1$ and

$$
\hat{\lambda}_{j}=\left(\sum_{k=1}^{\mathrm{K}} \mathrm{d}_{j k}\right) /\left(\sum_{k=1}^{\mathrm{K}} t_{j k}\right)
$$

the maximum likelihood estimator of the stratum specific failure rate under the null hypothesis of no trend across exposure. The $x_{k}$ are quantitative variables representing. exposure levels. Again plugging in the classical $v_{j \mathrm{k}}$ in (4.6) and (4.8) results in the classical variance formulas.

\subsection{Tests for heterogeneity of relative rates}

To test for a general difference among the rate ratios in the $\mathbf{J}$ strata or to test the global null hypothesis that failure: rates for none of the $\mathrm{K}$ exposure classes differ (that is $\boldsymbol{\Psi}_{\mathrm{k}}=\mathrm{I}$ for $\mathrm{k}=1,2 \ldots, \mathrm{K}$ ) the usual chi-square tests, can be used. The classical chi-square tests remain chi-square with the usual degrees of freedom on substituting the relevant case-cohort quantities from table 2 in the formulas.

To test for a general difference among the rate ratios in the $\mathrm{J}$ strata we can use:

$$
\chi_{J-1}^{2}=\sum_{j=1}^{J}\left[\frac{\left(d_{j 1}-\lambda_{j} t_{j 1}\right)^{2}}{\hat{\lambda}_{j} t_{j 1}}+\frac{\left(d_{j k}-\hat{\varphi}_{k} \lambda_{j} t_{j k}\right)^{2}}{\Psi_{k} \hat{\lambda}_{j} t_{j k}}\right]
$$


where $\hat{\psi}_{k}$ and $\hat{\lambda}_{j}=\left(d_{j 1}+d_{j k}\right) /\left(t_{j 1}+\hat{\psi}_{k} t_{j k}\right)$ are the same maximum likelihood estimators used in formulas (4.5) and (4.6).

To test the global null hypothesis that $\boldsymbol{\psi}_{k}=1, k=1,2, \ldots, \mathrm{K}$ we can use:

$$
x_{k-1}^{2}=\sum_{k=1}^{x}\left[\frac{\left(\sum_{j=1}^{J} d_{j k}-\sum_{j=1}^{J} \lambda_{j} t_{j k}\right)^{2}}{\left(\sum_{j=1}^{J} \lambda_{j} t_{j k}\right)}\right]
$$

with $\hat{\psi}_{k}=1$ and

$$
\hat{\lambda}_{j}=\left(\sum_{k=1}^{x} d_{j k}\right) /\left(\sum_{k=1}^{x} t_{j k}\right)
$$

Both statistics have asymptotic chi-square distributions with $\mathrm{J}-1$ and $\mathrm{K}-1$ degrees of freedom, respectively.

However, the test $(4.10)$ for the null hypothesis $\downarrow_{k}=1, k=1,2, \ldots, K$ is conservative compared to the score test for the same hypothesis (see ref. 12, page 114). The formula for the score test is, using notation as in Breslow and Day (12):

$$
\chi_{\mathrm{K}-1}^{2}=(O-E)^{\mathrm{T}} M^{-1}(O-E)
$$

and this statistic has an asymptotic chi-square distribution with $\mathrm{K}-1$ degrees of freedom.

$\mathrm{O}$ is a $\mathrm{K}-1$ dimensional vector $\left(\mathrm{O}_{2}, \mathrm{O}_{3}, \ldots, \mathrm{O}_{\mathrm{K}}\right)^{\mathrm{r}}$ with components

$$
o_{k}=\sum_{j=1}^{J} d_{j k}, k=2,3, \ldots, k
$$

$\mathrm{E}$ is a $\mathrm{K}-1$ dimensional vector $\left(\mathrm{E}_{2}, \mathrm{E}_{3}, \ldots, \mathrm{E}_{\mathrm{K}}\right)^{\mathrm{T}}$ with

$$
E_{k}=\sum_{j=1}^{J} \lambda_{j} t_{j k}, k=2,3, \ldots, k
$$

$M$ is a $(K-1)$ by $(K-1)$ dimensional matrix that can be represented as a sum of $J(K-1)$ by $(\mathrm{K}-1)$ dimensional matrices

$$
M=\sum_{j=1}^{J} M_{j}
$$

Each matrix $M_{j}$ has diagonal elements $v_{j k}\left(v_{j}-v_{j k}\right) / v_{j}$ (with $k=2,3, \ldots, K$ ) and off diagonal elements $-v_{j k} v_{j} / v_{j}$ (with $2 \leq k \leq K$ and $2 \leq 1 \leq K$ ), with $v_{j}=\Sigma v_{j k}$, where the summation is over $\mathbf{k}$ from 1 to $\mathbf{K}$.

The $E_{k}$ and the $v_{j k}$ are calculated using $\hat{\phi}_{k}=1$ and

$$
\lambda_{j}=\left(\sum_{k=1}^{K} d_{j k}\right) /\left(\sum_{k=1}^{K} t_{j k}\right)
$$

Plugging in the classical $\mathrm{v} 2_{j \mathrm{k}}$ again gives the classical formula for $\mathbf{M}$. 


\section{Regression methods for grouped and individual case-cohort data}

In $\$ 4$ we presented case-cohort set-up adaptations of the well known elementary statistical methods. These methods presumed a summarization of the data in a twodimensional table with $\mathrm{J}$ rows, the strata, and $\mathbf{K}$ columns, the exposure classes. Each cell of such a table contains the basic data: the failure counts $d_{j}$ and the case-cohort equivalents of the person years denominators $t_{j k}$. The stratification variables will in general be nuisance factors known to have an effect on the baseline rates but with only secondary importance. The real problem is to describe the effects of the exposure variables, and their possible modification by the effects of the stratification variables, in explicit detail.

Regression analyses offers a more versatile approach than these elementary methods and is easily initiated by adding to the basic data $\left(\mathrm{d}_{j \mathrm{k}}, \mathrm{t}_{\mathrm{j}}\right)$ in each cell of the table a pdimensional row vector $x_{j \mathrm{k}}=\left(\mathrm{x}_{\mathrm{j} k 1}, \ldots, \mathrm{x}_{\mathrm{jkp}}\right)$ of regression variables. These may represent either qualitative or quantitative coding of degree, intensity and/or duration of possibly different exposure variables, nuisance (stratification) variables and interactions among exposure variables and/or interactions between exposure and nuisance variables. The goal of the regression analysis is to try to disentangle the separate effects of exposure and nuisance variables, the $x_{1}, \ldots, x_{p}$, on these rates by introducing different parametrizations to represent possible effects. A number of structures have been proposed for the rates of which the additive (excess) and multiplicative (relative) rate models are the most common.

We shall here concentrate on the multiplicative rate model as this seems to be the modell that is by far the most frequently used. The multiplicative model implies that the cell-specific rate

$$
\lambda_{j k}=\exp \left(\tilde{x}_{j k}^{T} \beta_{1}\right)=\exp \left(\boldsymbol{\beta}_{0}+x_{j k 1} \boldsymbol{\beta}_{1}+\ldots+x_{j k p} \boldsymbol{\beta}_{p}\right)
$$

is an exponential relative rate function $\exp \left(\mathrm{x}_{j k 1} \beta_{1}+\ldots+\mathrm{x}_{j \mathrm{k}} \beta_{\mathrm{p}}\right)$ times a baseline rate $\lambda_{0}=\exp \left(B_{0}\right)$ where $\tilde{x}_{j k}$ stands for $\left(1, x_{j, 1}, x_{j k 2}, \ldots, x_{j k p}\right)^{\mathrm{T}}$.

Other structures are discussed by Volovics (15).

Given a data matrix as above, regression analysis can be based on a 'pseudo partiallikelihood' function. For example, for the 'blow-up' approach:

$$
L(\beta)=L\left(\beta_{0}, \beta_{1}, \ldots, \beta_{D}\right)=\prod_{k=1}^{K} \prod_{j=1}^{J} \lambda_{j k}^{d_{j k}} e^{-\lambda_{j k} t_{j k}}
$$

where $\lambda_{j k}=\exp \left(\bar{x}_{j k}{ }^{\top} \beta\right)$ and the $d_{j k}$ and $t_{j k}$ are taken from table 2 .

The expression is a pseudolikelihood because it does, not represent the 'likelihood" of the data given the sampling design (it does exhibit properties more or less similar to those of a proper likelihood function) and it is a partial likelihood because it does not contain a factor describing the conditional distribution of censoring or the distribution of $\mathrm{x}_{\mathrm{jk}}$.

Using the formal resemblance of the pseudolikelihood to a likelihood for a Poisson process, any software package with facilities for Poisson regression can be used to estimate $B$ and to test hypotheses with respect to B (likelihood ratio, score oi Wald tests). However, as will be clear from the presentation of the elementary methods above, the estimate of the asymptotic covariance matrix of the estimator for B will have 
to be adapted to account for case-cohort sampling. To calculate the case-cohort adapted estimate of the asymptotic covariance matrix we need quantities $\mathrm{v}_{\text {ik }}$ (analogous to the $v_{j k}$ specified in table 2) using formulas (3.3) or (3.4) but with $\hat{\lambda}_{j \mathrm{k}}=\exp \left(\bar{x}_{j k}{ }^{T} B\right)$,

$B$ the maximum likelihood estimate of $B$, substituted instead of the $\lambda_{j}$ and $\hat{\psi}_{\mathbf{k}}$ from the elementary analyses situation. This means that after estimating $B$ from the grouped data we would have to go back to the data on the individual level to adjust the covariance estimates or to prepare in advance a table containing other (and more) quantities than the $v_{i k}$. This does not seem very practicable and we will therefore only present exponential failure time regression analysis for data on the individual level. (Grouped data analyses can then easily be accomplished by defining dummy variables or scores for levels of strata or levels of exposure variables).

The analyses are based on a pseudo partial-likelihood function for the individual data with individuals denoted by $\mathrm{i}(\mathrm{i}=1,2, \ldots, \mathrm{n})$, which we present separately for the 'shrink' and 'blow-up' approaches:

$$
L(\beta)=\prod_{i=1}^{\text {II }} \mu_{i}^{d_{i}} e^{-\mu_{1} m_{1} t_{1}}
$$

$$
L(\beta)=\prod_{1=1}^{n}\left(\lambda_{1} e^{-\lambda_{1} t_{1}}\right)^{d_{1}}\left(e^{-\lambda_{1} s_{1} t_{1}}\right)^{p^{-1}\left(1-d_{1}\right)}
$$

with $\mathrm{d}_{\mathrm{i}}, \mathrm{t}_{\mathrm{i}}$ and $\mathrm{s}_{\mathrm{i}}$ as in $\$ 2, \mu_{\mathrm{i}}=\rho^{-1} \lambda_{\mathrm{i}}, \lambda_{\mathrm{i}}=\exp \left(\overline{\mathrm{x}}_{\mathrm{i}}^{\mathrm{T}} \beta\right)$.

When using the 'shrink' likelihood one should keep in mind that given $\lambda_{1}=\exp \left(\beta_{0}+x_{i j} \beta_{1}+\ldots+x_{i p} \beta_{p}\right)$ this approach always estimates $\mu_{\mathrm{i}}=\rho^{-1} \lambda_{\mathrm{i}}=\exp \left(\alpha_{0}+x_{i \mathrm{i}} \beta_{1}+\ldots+x_{i p} \beta_{\mathrm{p}}\right)$ with $\alpha_{0}=\beta_{0}-\log \rho$ : the baseline failure rate $\exp \left(\beta_{0}\right)$ is 'blown-up' to $\rho^{-1} \exp \left(\beta_{0}\right)=\exp \left(\alpha_{0}\right)$. The relative rates $\exp \left(\mathrm{x}_{i j} \beta_{\mathrm{j}}\right)$, however, are correctly estimated, see the heuristic argument in $\$ 2$.

Any software package with facilities for exponential failure time regression or, in some cases, Poisson regression or nonlinear regression, can be used to get a maximum likelihood estimate $\beta$ of $B$ and thus an estimate $\hat{\lambda}_{i}$ of $\lambda_{i}(13)$. Likelihood ratio tests for hypothesis about $B$ or $B$ components are also easily calculated. For confidence intervals or for Wald or score tests, however, the case-cohort adapted estimates of the covariance matrix of $\bar{B}$ will be needed. Software with macro or programming facilities and matrix operations will be needed for this. GAUSS, GLIM, S, and SAS with IML for example, are excellent. Let $X$ stand for the $n$ by $(p+1)$ data matrix with rows $\bar{x}_{i}=\left(1, x_{i 1}, \ldots, x_{i p}\right)$. Let $C$ stand for the covariance matrix of $\beta$ estimated by the regression program. Then the estimate of the case-cohort adjusted covariance matrix can be written as $\mathbf{C \Delta C}$ where the matrix $\Delta$ is estimated differently depending on which approach, 'shrink' or 'blowup', is used. We will denote these estimates $\Delta_{\mathrm{S}}$ and $\boldsymbol{\Delta}_{\mathrm{B}}$. Again just as in $\$ 3$ we present two estimates of $\Delta_{5}$ and $\Delta_{\mathrm{B}}$, namely:

$$
\Delta 1_{s}=x^{T} \operatorname{Diag}\left(\left(d_{1}-\hat{\mu}_{1} s_{1} t_{1}\right)^{2}\right) x
$$

$$
\Delta 1_{B}=X^{T} \operatorname{Diag}\left(\left(d_{1}-\lambda_{1}\left(d_{1} t_{1}+\left(1-d_{1}\right) s_{1} \rho^{-1} t_{1}\right)\right)^{2}\right) X
$$




$$
\Delta 2_{B}=x^{\top} \operatorname{Diag}\left(\lambda_{1}\left(d_{1} t_{1}+\left(1-d_{1}\right) s_{1} \rho^{-1} t_{1}\right)+(1-\rho)\left(1-d_{1}\right) s_{1} \lambda_{1}^{2}\left(\rho^{-1} t_{1}\right)^{2}\right) x
$$

where the notation $\operatorname{Diag}\left(\mathrm{w}_{\mathrm{i}}\right)$ stands for an $\mathrm{n}$ by n matrix with $\mathbf{w}_{1}, \mathbf{w}_{2 \mathrm{a}}, \ldots, \mathbf{w}_{\mathrm{n}}$ on the diagonal and the off-diagonal elements equal to 0 . The matrices $\Delta 1$ and $\Delta 2$ contain the equivalents, in the context of regression based on individual subjects, of the $\mathrm{vl}_{\mathrm{jk}}$ and $\mathrm{v}_{\mathrm{jk}}$ introduced in $\$ 3$. The $v 1_{j \mathrm{k}}$ and $\mathrm{v} 2_{\mathrm{k}}$ can in principle be calculated through $\Delta 1$ and $\Delta 2$ after defining relevant likelihood functions based on stratum-exposure specific rates and using a data matrix $\mathrm{X}$ containing variables to define strata and exposure.

If it is suspected that the failure rate is not approximately constant for the study duration it is advisable to fit a piecewise exponentiall distribution to the failure times. This is achieved by choosing a set of time points $a_{1}<a_{2}<\ldots<a_{L-1}$ with $0=a_{0}<a_{1}$ and $\mathrm{a}_{\mathrm{L}-1}<\mathrm{a}_{\mathrm{L}}=\infty$ or $\mathrm{a}_{\mathrm{L}}=\tau$ with $\tau$ the maximal possible observation time for any individual under study. In each interval $\left(a_{\ell-1}, a_{\ell}\right]$ we model an individual hazard function $\lambda_{i}(\mathbf{t})$ as a constant $\lambda_{i c}$. Given a multiplicative rate model as used above, this means defining a piecewise constant baseline failure rate common to all subjects i.e. $\lambda_{0 \ell}=\exp \left(\beta_{0 R}\right)$ on $\mathrm{a}_{\ell-1}<\mathbf{i} \leq \mathrm{a}_{\ell}, \mathrm{l}=1,2, \ldots, \mathrm{L}$.

Then we have as individual failure rate $\lambda_{i c}=\lambda_{0 \ell} \exp \left(x_{i}^{\top} \beta\right)=\exp \left(\beta_{0 \ell}+x_{i}^{\top} \beta\right)$. Note that the 'shrink' approach estimates a blown up baseline failure rate on each interval $\mathrm{a}_{\ell-1}<\mathrm{t} \leq \mathrm{a}_{\ell}$, namely $\mu_{0 \ell}=\rho^{-1} \exp \left(\beta_{0 \ell}\right)=\exp \left(\beta_{0 \ell}-\log \rho\right)=\exp \left(\alpha_{0 \ell}\right)$.

If we consider failure for each interval $\left(a_{t-1}, a_{\ell}\right]$ separately, then the $i$-th subject experiences a sequence of censorings at $a_{1}, a_{2}, \ldots$ until final censoring or failure at $t_{i}$ defined to fall in the $\mathrm{L}_{\mathrm{i}}$-th interval, so that $\mathrm{a}_{\mathrm{Li}^{-1}}<\mathrm{t}_{\mathrm{i}} \leq \mathrm{a}_{\mathrm{L}_{\mathrm{i}}}$.

Define for every subject a sequence of failure indicators $d_{i 1}, d_{i 2}, \ldots, d_{i L}$ with $d_{i l}=0$ for $1 \leq \ell<L_{i}$ and $d_{i L i}=1$ or 0 depending on whether $i$ failed or was censored at $t_{i}$ and $a$ sequence of failure (exposure) times $t_{i 1}, t_{i 2}, \ldots, t_{i L}$ with $t_{i \ell}=a_{\ell}-a_{\ell-1}$ for $1 \leq \ell<L_{1}$ and $\mathrm{t}_{\mathrm{iL}}=\mathrm{t}_{\mathrm{i}}-\mathrm{a}_{\mathrm{L}_{\mathrm{i}}-1}$.

When the 'blow-up' approach is being used it is easiest to blow-up failure times of censored individuals $\left(d_{i}=0\right)$ beforehand, i.e. to use $p^{-1} t_{i}$ instead of $t_{i}$. Then $L_{i}$ is defined by $a_{L_{i}-1}<\rho^{-1} t_{i} \leq a_{L}$. The $d_{i e}$ and $t_{i e}$ for such individuals are adapted accordingly.

Then the pseudolikelihood functions (5.1 S) and (5.1 B) become:

$$
L(\beta)=\prod_{1=1}^{\mathrm{n}} \prod_{1=1}^{L_{1}} \mu_{11}^{d_{11}} e^{-\mu_{11} a_{1} t_{11}}
$$

$$
L(\beta)=\prod_{i=1}^{n} \prod_{1=1}^{L_{1}}\left(\lambda_{11} e^{-\lambda_{11} t_{11}}\right)^{d_{11}}\left(e^{-\lambda_{11} n_{1} t_{11}}\right)^{\left(1-d_{12}\right)}
$$

The pseudolikelihood for the 'blow-up' approach (5.4 B) is written without an exponent $\rho^{-1}$ in the second factor here (compare (5.1 B)) because the $t_{i c}$ are defined using $\rho^{-1} t_{\mathrm{t}}$. To fit such piecewise exponential models, the data matrix has to be augmented to contain $\mathrm{L}_{1}$ rows for each individual i (see Aitkin et al. (13), section 6.22). This means that the total number of data matrix rows equals $\mathrm{L}$ 
where

$$
L=\sum_{i=1}^{\mathrm{n}_{1}} \mathrm{~L}_{1}
$$

If $\mathrm{L}_{\mathrm{i}}$ and $\tilde{\mathrm{n}}_{\mathrm{i}}$ are at all large this might pose problems for some software packages and machines, and also the computation time might become prohibitive. When fitting the regression model based on $(5.4 \mathrm{~S})$ or $(5.4 \mathrm{~B})$ we treat each row of the augmented data matrix as an independent subject and proceed as if fitting the models (5.1 S) or (5.1 B). The necessary adaptations of the covariance matrix estimates for case-cohort studies are easily obtained with formulas $(5.2 \mathrm{~S})$ to $(5.3 \mathrm{~B})$, replacing $\mathrm{X}$ by the augmented data matrix of size $\mathbf{L}$ by $(\mathrm{p}+1)$ and $\mathrm{d}_{\mathrm{i}}, \hat{t}_{\mathrm{i}}, \hat{\lambda}_{\mathrm{i}}, \hat{\boldsymbol{\mu}}_{\mathrm{i}}$ by $\mathrm{d}_{\mathrm{i} \ell}, \hat{t}_{i \ell}, \hat{\lambda}_{\mathrm{i} \ell}, \hat{\mu}_{\mathrm{i} \ell}$. For further details on fitting piecewise exponential distribution models and also on fitting time-dependent covariates (which follows the same procedures) see Aitkin et al. (13), sections 6.15, 6.16, 6.17 and 6.22 .

\section{$6 \quad$ Illustrative analysis}

The data for this illustration come from a prospective cohort study on diet, life-style and cancer that was started in the Netherlands in 1986. The cohort included 58,279 men and 62,573 women aged 55-69 years at the start of the study. At baseline, cohort members completed a self-administered questionnaire on dietary habits, potential confounders and other independent risk factors for cancer such as smoking habits, occupation and education. Following the case-cohort approach, a subcohort of 3,500 subjects was randomly sampled from the cohort after the baseline exposure measurement. The subcohort has been followed up biennially for vital status information in order to estimate the accumulated persontime in the cohort (16). Incident cancer cases occurring in the cohort have been identified by record linkage to cancer registries and a pathology register (17). This illustrative analysis pertains to the lung cancer incidence in the recently completed 3.3 year follow-up period. In this period a total of 617 cases of lung cancer were detected in the total cohort of 120,852 subjects. After excluding incident cases with in situ carcinoma, cases whose diagnosis was not microscopically confirmed and cases who reported a history of cancer other than skin cancer in the baseline questionnaire, 552 incident cases of lung cancer were available for analysis. After excluding prevalent cancer cases other than skin cancer from the subcohort of 3500 as well, 3346 subjects remained in this group.

For this illustration, the relationship between smoking habits (categorized as never/ex/current smokers) and lung cancer risk was analyzed with the proposed methods of case-cohort analysis. To be concise, we only present results here on relative rate estimation, confidence intervals, Mantel-Haenszel tests and test for trend in relative rates across exposure categories. In the stratified analysis, we stratified for gender and age (in three five-year categories). This was followed by relative rate regression analysis using the individual data, while adjusting for gender and age (again in 3 categories for reasons of comparability with the stratified analysis). All analyses were conducted using the 'shrink' method and the 'blow-up' method of estimating the person years. Table 3 shows the basic quantities that were computed for the stratified smoking-lung cancer analyses. In this table (with the same format as table 2), the quantities for the 'shrink' and 'blow-up' method have been specified. 
Tabie 3. Grouped data for stratified analysis of case-cohort study on smoking and lung cancer.

\begin{tabular}{|c|c|c|c|c|c|c|c|c|}
\hline \multirow[b]{2}{*}{$\begin{array}{l}\text { Stratum } \\
\text { (j) }\end{array}$} & \multirow[b]{2}{*}{$\begin{array}{l}\text { Exposure" } \\
\text { (k) }\end{array}$} & \multirow[b]{2}{*}{$\begin{array}{l}\text { Failures } \\
\left(d_{p_{p}}\right)\end{array}$} & \multicolumn{3}{|c|}{ Shrink method } & \multicolumn{3}{|c|}{ Blow-up method } \\
\hline & & & $\begin{array}{l}\text { Person } \\
\text { years } \\
\left(t_{\text {W }}\right)\end{array}$ & $\begin{array}{l}\text { Variance1 } \uparrow \\
\left(\mathrm{vi}_{\mu}\right)\end{array}$ & $\begin{array}{l}\text { Variance } 2 \dagger \\
(\sqrt{2}, j)\end{array}$ & $\begin{array}{l}\text { Person } \\
\text { years } \\
(1,2)\end{array}$ & $\begin{array}{l}\text { Variancel } 1 \\
\left(\mathrm{v1}_{\hat{\beta}}\right)\end{array}$ & $\begin{array}{l}\text { Variance } 2 \uparrow \\
\left(V_{2}, \mu\right)\end{array}$ \\
\hline \multirow[t]{3}{*}{1 (55-69 y, men) } & I Never & $\mathbf{0}$ & 209 & 0.00 & 0.00 & 7227 & 0.00 & 0.00 \\
\hline & $2 \mathrm{Ex}$ & 32 & 925 & 35.08 & 33.33 & 31920 & 35,13 & 33.32 \\
\hline & 3 Current & 87 & 873 & 113.61 & 111.43 & 30195 & 113.17 & 111.31 \\
\hline \multirow[t]{3}{*}{2 (55-59 y, women) } & 1 Never & 0 & 1092 & 0.00 & 0.00 & 37710 & 0.00 & 0.00 \\
\hline & $2 \mathrm{Ex}$ & 3 & 490 & 3.02 & 1.91 & 16925 & 3.02 & 1.91 \\
\hline & 3 Current & 16 & 545 & 17.10 & 14.56 & 18846 & 17.05 & 14.56 \\
\hline \multirow[t]{3}{*}{3 (60-64 y. men) } & 1 Never & 1 & 167 & 1.02 & 1.02 & 5765 & 1.02 & 1.02 \\
\hline & $2 \mathrm{Ex}$ & 51 & 854 & 60.35 & 58.52 & 29564 & 59.99 & 58.46 \\
\hline & 3 Current & 119 & 787 & 174.26 & 173.14 & 27154 & 174.05 & 172.87 \\
\hline \multirow[t]{3}{*}{4 ( $60-64$ y, women) } & 1 Never & 6 & 1151 & 6.09 & 6.10 & 39692 & 6.10 & 6.10 \\
\hline & $2 \mathrm{Ex}$ & 4 & 338 & 4.27 & 5.52 & 11678 & 4.26 & 5.52 \\
\hline & 3 Current & 12 & 392 & 13.66 & 15.77 & 13562 & 13.61 & 15.78 \\
\hline \multirow[t]{3}{*}{5 (65-69 y, men) } & 1 Never & 6 & 136 & 6.87 & 6.84 & 4705 & 6.84 & 6.84 \\
\hline & 2. $\mathrm{Ex}$ & 63 & 722 & 82.57 & 84.72 & 25028 & 81.80 & 84.56 \\
\hline & 3. Current & 124 & 556 & 213.96 & 218.57 & 19092 & 215.38 & 218.51 \\
\hline \multirow[t]{3}{*}{6 (65-69 y, women) } & 1 Never & 7 & 1083 & 7.15 & 7.14 & 37420 & 7.14 & 7.14 \\
\hline & 2. Ex & 4 & 269 & 434 & 5.65 & 9279 & 4.33 & 5.64 \\
\hline & 3 Current & 10 & 217 & 12.03 & 1359 & 7504 & 11.96 & 13.60 \\
\hline
\end{tabular}

-) Smoking categories.

†) Cell-specific variances used for the estimation of the variance of the Mantel-Hacnszel relative rate.

In table 4 (panel $A$ ), the results of the stratified analyses are shown for both the shrink. and the blow-up approach. As expected, the relationship between smoking and lung. cancer is very strong. Compared to never-smokers, the Mantel-Haenszel relative rate estimates, for ex-smokers and for cuirent smokers are 3.77 and 10.79 , respectively, using the shrink method. The RR estimates obtained with the blow-up method are virtually identical.

The $95 \%$ confidence intervals shown are constructed using the first variance estimates (formula 4.2 , using $v 1_{j k}$ ). The first and second estimate of $\operatorname{var}\left(\log R R_{M H}\right)$ were very close to each other: for the contrast between ex- and never smokers the two estimates of $\operatorname{var}\left(\log \mathrm{RR}_{\mathrm{MH}}\right)$ were 0.0876 and 0.0889 , respectively, while for the contrast between current and never smokers these variances estimates were 0.0849 and 0.0831 , respectively (using the shrink method). Both relative rate estimates were significantly different from 1: the Mantel-Haenszel $\chi^{2}$-test values were 28.60 and 109.38 for the respective contrasts in smoking habits (ex vs. never; current vs. never smoking). The $\chi^{2}$ test for trend was also highly significant. The variance estimates and $\chi^{2}$-test values were again similar when using the blow-up method instead of the shrink method.

Table 4 (panel B) also shows the results of the relative rate regression analysis with the individual data. When an exponential distribution of failure times is assumed (i.e., a constant hazardi), the association between smoking status and lung cancer is estimated essentially similar to the stratified analyses. (Again, the presented confidence intervals are based on the first variance estimate (formula 5.2).) 
Table 4. Results of stratified analysis and relative rate regression analysis for case-cohort study on smoking and lung cancer.

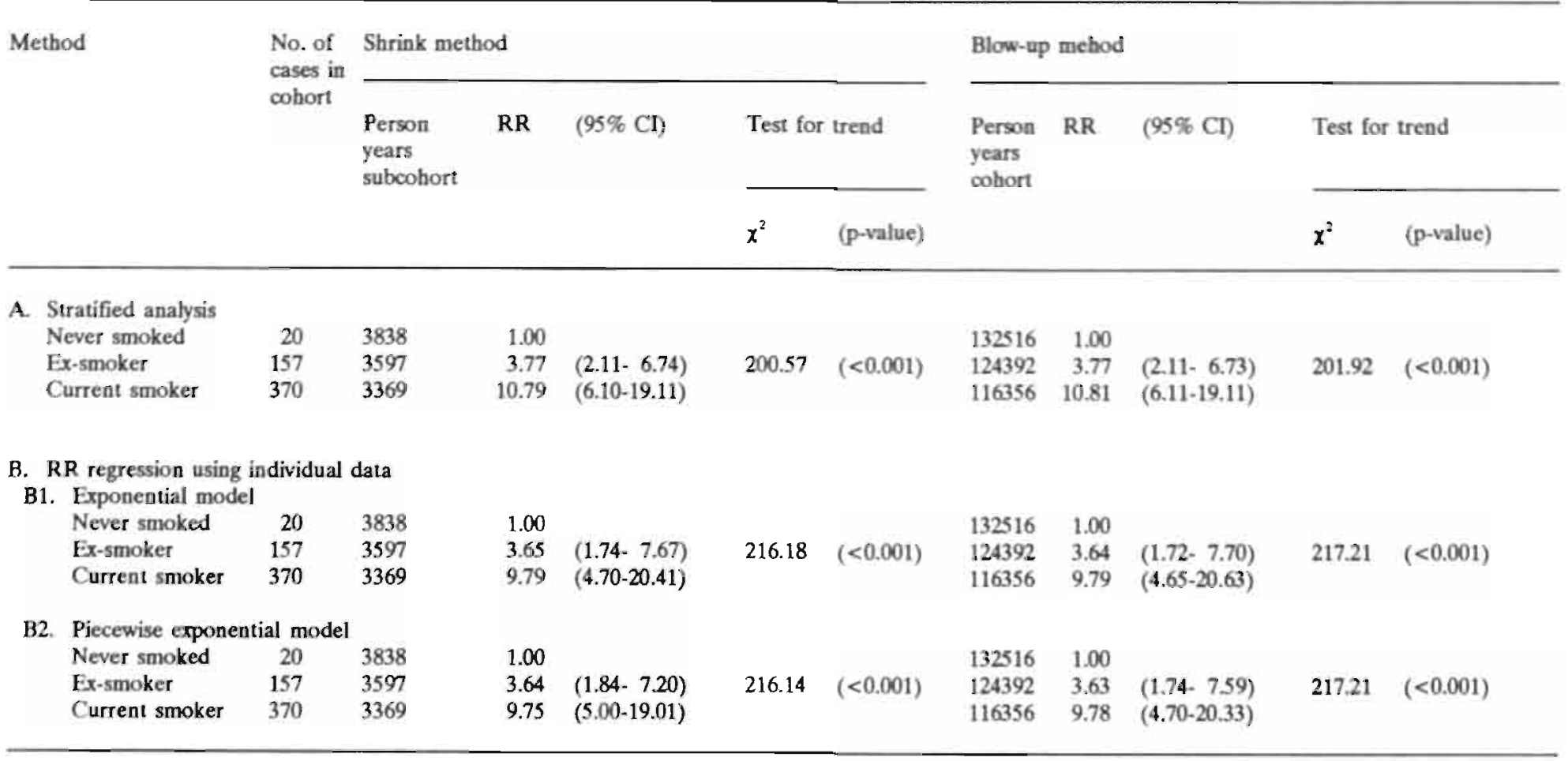


It should be mentioned that we compare here the Mantel-Haenszel relative rate of the stratified analysis with the maximum likelihood estimate of the regression analysis. Also, the tests for trend are results from the score test and the likelihood ratio test, respectively. As an aside, when uncorrected variance estimates would have been used, the $95 \%$ confidence intervals would have been more narrow, with limits $(2.25,5.93)$ and (6.12, 15.66) for the respective exposure contrasts (with the shrink method). Again, there is no difference in relative rate estimates, confidence intervals and trend tests between the shrink and blow-up approach. When a piecewise exponential distribution is assumed (with constant hazards per year of follow-up in the 3.3 year period), the results are similar to the situation where an exponential distribution is assumed. Thus, the assumption of a constant hazard during the 3.3 years of follow-up is justified.

In conclusion, the strong positive dose-response relationship between smoking and lung cancer is reproduced in this case-cohort analysis. With two covariates in the model, the stratified analysis and the regression analyses yield essentially similar results. The advantage of the relative rate regression model using individual data is that it can easily be extended to more covariates.

\section{Discussion}

Given the availability of GLIM-macros, the described case-cohort analysis methods (which are adaptations of the standard tocils of epidemiologic practice) are easy to use. We have chosen to analyze as an example data on the relationship between smoking and lung cancer to demonstrate that these method's reproduce results close to the relatively stable relative risks of lung cancer known for smoking exposure. Ideally this illustration should have been complemented by simulation studies or by a comparison of the results of a case-cohort analysis with those from a classical analysis of an original, full cohort study, but we have not yet had the opportunity to attempt these timeconsuming activities.

There seems to be no clear reason for choosing between the 'shrink' and the 'blow-up' approach. In the various exposure-disease relationships analyzed so far in our cohort study on diet and cancer, the results of the 'shrink' approach and the 'blow-up' approach are very close. It seems slightly more natural to use only subcohort person years instead of the (crudely) estimated cohort person years but this subjective preference is not reflected in the performance of the methods. With respect to the two possible variance estimators for the asymptotic variances of the statistics (those based on the $v 1_{j k}(\Delta 1)$ and those based on the $\left.v 2_{j k}(\Delta 2)\right)$, the following can be mentioned. Both variance estimators are consistent estimates of the same parameters and thus asymptotically equivalent. We can only judge the small sample behavior on the basis of the various exposure-disease relationships analyzed so far; the variance estimates based on the $v 2_{j k}(\Delta 2)$ appear to be somewhat more variable than those based on the $v 1_{j k}$ ( $\Delta 1)$. Furthermore the agreement between the two methods is less than that between the 'shrink' and the 'blow-up' approach. On the basis of results obtained so far we suggest using variance estimates based on the $v 1_{j k}(\Delta 1)$, but a definite conclusion will have to await the results of simulation studies.

In the illustration we showed that the assumption of a constant hazard in the $\mathbf{3 . 3}$ years of follow-up was justified, after comparing it with results from the piecewise exponential model with a year-specific hazard. We used the years of follow-up as cutpoints for the piecewise modelling; a further refinement of the cutpoints is possible, eventually 
resulting in cutpoints defined by the individual failure times. The use of such cutpoints would result in estimates that are close to the estimates obtained by fitting a Cox proportional hazards model (Aitkin et al. (13), sections; 6.15 and 6.16). With a size of the case-cohort study that we are conducting, the use of these detailed cutpoints would computationally be very burdensome, however.

\section{References}

1. Liddell FDK, McDonald JC, Thomas DC. Methods for cohort analysis: appraisal by application to asbestos mining (with discussion). J R Statist Soc A 1977; 140: 469-490.

2. Prentice RL. A case-cohort design for epidemiologic cohort studies and disease prevention trials. Biometrika 1986; 73: 1-11.

3. Goldstein L, Langholz. B. Asymptotic theory for nested case-control sampling in the Cox regression model. Ann Statist (in press) 1992.

4. Prentice RI. On the design of synthetic case-control studies. Biometrics 1986; 42: 301-310.

5. Self SG, Prentice RL. Asymptotic distribution theory and efficiency results for case-cohort studies. Ann Statist 1988; 16: 64-81.

6. Lubin JH, Gail MH. Biased selection of controls for case-control analyses of cohort studies. Biometrics 1984; 40: 63-75.

7. Robins JM, Gail MH, Lubin JH. More on 'Biased selection of controls for case-control analyses of cohori studies'. Biometrics 1986; 42: 293-299.

8. Wacholder S, Boivin JF. External comparisons with the case-cohort design. Am J Epidemiol 1987; 126: 1198-1209.

9. Robins JM, Prentice RL, Blevins D. Designs for synthetic case-control studies in open cohorts. Biometrics 1989; 45: 1103-1116.

10. Langhoz. B, Thomas DC Nested case-control and case-cohort methods of sampling from a cohort: a critical comparison. Am J Epidemiol 1990; 131: 169-176.

11. Wacholder S, Gail MH, Pee D, Brookmeyer R. Alternative variance and efficiency calculations for the case-cohorı design. Biometrika 1989: 76: 117-123.

12. Breslow NE, Day NE. Statistical Methods in Cancer Research. II. The Design and Analysis of Cohort Studies. Lyon: IARC Sci Publ: 1987; 82.

13. Aitkin $M$, Anderson D, Francis B, Hinde J. Statistical Modelling in GLIM. Oxford: Oxford University Press, 1989.

14. Andersen PK, Borgan Ø, Gill RD, Keiding N. Statistical Models based on Counting Processes. New York: Springer Verlag, 1992.

15. Volovics A. Simple methods for the analyses of case-cohort studies. In preparation, 1992.

16. Yan den Brandi PA, Goldbohm RA, Van 't Veer P, Volovics A, Hermus RJJ, Sturmans F. A large-scale prospective cohort study on diet and cancer in The Netherlands. J Clin Epidemiol 1990; 43: 285-295.

17. Van den Brandt PA, Schouten IJ, Goldbohm RA, Dorant E, Hunen PMH. Development of a record linkage protocol for use in the Dutch cancer registry for epidemiological research. Int I Epidemiol 1990; 19: 553-558.

18. Borgan $\varnothing$. Maximum liketihood estimation in parametric counting process models, with applications to censored failure time data. Scand J Statist 1984: 11: 1-16.

19. Andersen PK, Gill RD. Cox's regression model for counting processes. A large sample study. Ann Siatist 1982: 10: 1100-1120.

20. Lehmann EL. Testing Statistical Hypotheses. New York: Wiley, 1959. 


\section{Appendix}

Proofs of all results mentioned in this paper can be obtained by applying the results of Borgan (18) and Self and Prentice (5). Here we only sketch the ideas behind the proofs, full details and formulations of necessary assumptions and conditions can be found in Volovics (15). Given a cohort study comprising $\mathrm{n}$ individuals, J strata and $\mathrm{K}$ exposure classes we have denoted the stratum-exposure specific failures and person years with $d_{j k}$ and $t_{j k}$. When a case-cohort study is derived from this cohort based on a sample of $\mathbf{m}$ of the $\mathbf{n}$ cohort members we shall denote both 'shrink' and 'blow-up' stratum-exposure specific person years by $\bar{t}_{j \mathrm{k}}$. For the 'shrink' approach $\mathbf{t}_{j \mathrm{k}}=\mathbf{\Sigma}_{\mathrm{s}_{\mathrm{i}}} \mathrm{t}_{\mathrm{i}}$ and for the 'blow-up' approach $i_{i k}=\Sigma\left[d_{i} t_{i}+\left(1-d_{i}\right) \rho_{n}{ }^{-1} s_{i} t_{i}\right]$ where the summation is over all individuals $\mathrm{i}$ in the stratum-exposure combination $(\mathrm{j}, \mathrm{k})$ and we here write $\rho_{\mathrm{n}}$ for the sampling fraction $\mathrm{m} / \mathrm{n}$. Write $\theta_{\mathrm{jk}}$ for the stratum-exposure specific rates.

From a multivariate version of the martingale central limit theorem (e.g., ref. 19, appendix I) it follows easily and under rather mild regularity conditions, that the random vector with components $\mathrm{n}^{-1 / 2}\left(\mathrm{~d}_{\mathrm{jk}}-\theta_{\mathrm{jh}} \mathrm{t}_{\mathrm{j}}\right)$. converges in distribution to a vector with components which are independent and normally distributed with means zero and variances $\theta_{j k} \mathbf{r}_{j \mathbf{k}}$, where it is assumed that $\mathbf{n}^{-1} \mathbf{t}_{j k}$ converges in probability to $\mathbf{r}_{j \mathrm{k}}>0$ (in short notation: $n^{-1} t_{j k}-->r_{j k}$ ). Writing $\theta_{j k}$ for the maximum likelihood estimates of $\theta_{j k}$ based on a cohort study, it follows from the results of Borgan (18) that the random vector with components $n^{1 / 2}\left(\partial_{j k}-\theta\right)$ converges in distribution to a vector with components which are again independently and normally distributed with means zero and variances $\theta_{j \mathrm{j}} / \mathrm{r}_{\mathrm{j}}$. If we parametrize $\theta_{i \mathrm{k}}$ as $\boldsymbol{\theta}_{j \mathrm{k}}=\lambda_{j} \downarrow_{k}$ or $\theta_{j \mathrm{k}}=\exp \left(\alpha_{i}+\beta_{k}\right)$ it again follows from Borgan (18) that the vector with cohort study maximum likellihood estimates $\left(\dot{\lambda}_{1}, \ldots, \dot{\lambda}_{1}, \hat{\psi}_{2}, \ldots, \hat{\psi}_{k}\right)$ or $\left(\hat{\alpha}_{1}, \ldots, \hat{\alpha}_{\mathrm{J}}, \beta_{2}, \ldots, \beta_{\mathrm{K}}\right)$ converges in distribution to a vector with a multivariate normal distribution with mean vector 0 and a certain covariance matrix $\Sigma$.

The maximum likelihood estimators are in all cases consistent estimators of their parameters. Returning for the moment to the rates $\boldsymbol{\theta}_{j k}$ we have for the 'shrink' approach that:

$$
\begin{aligned}
n^{-1 / 2}\left(d_{j k}-\rho_{n}^{-1} \theta_{j k} \tilde{t}_{j k}\right) & =n^{-1 / 2}\left(d_{j k}-\theta_{j k} t_{j k}\right)+\theta_{j k} n^{1 / 2}\left(n^{-1} t_{j k}-n^{-1} \rho_{n}^{-1} \tilde{t}_{j k}\right) \\
& =n^{-1 / 2}\left(d_{j k}-\theta_{j k} t_{j k}\right)+\theta_{j k} n^{1 / 2}\left(n^{-1} t_{j k}-m^{-1} \tilde{t}_{j k}\right)
\end{aligned}
$$

If it is additionally assumed that $\rho_{n} \cdots p>0$ and that $m^{-1} \bar{t}_{j k} \cdots>_{p} r_{j k}$ and given some further regularity conditions, then we have upon combining the martingale central limit theorem and the results of Self and Prentice (5. proposition 1) that the vector with components $n^{-1 / 2}\left(d_{j k}-\rho_{n}^{-1} \theta_{j k} i_{j k}\right)$ converges in distribution to a vector with components which are independently and normally distributed with means zero and variances. $\theta_{j k} \mathbf{I}_{j k}+\rho^{-1}(1-\rho) \theta_{j k}^{2} G_{j k}^{2}$ where $\rho^{-1}(1-\rho) \sigma^{2}$ is the asymptotic variance of $n^{1 / 2}\left(n^{-1} \mathbf{t}_{j k}-m^{-1} \bar{t}_{j k}\right)$ :

$$
\left(n^{-1 / 2}\left(d_{j k}-\rho_{n}^{-1} \theta_{j k} \tilde{t}_{j k}\right)\right) \quad->_{D} N\left(0,\left(\theta_{j k} r_{j k}+p^{-1}(1-\rho) \theta_{j k}^{2} \sigma_{j k}^{2}\right)\right)
$$

The variances $\theta_{j k} \mathrm{r}_{j \mathrm{k}}+\rho^{-1}(1-\rho) \theta_{j k}{ }^{2} \sigma_{j k}{ }^{2}$ can be consistently estimated by the quantities $v 2_{j k}$. It can also be shown that, given the necessary conditions,

$$
\lim \operatorname{Cov}\left(\mathrm{n}^{-1}\left(\mathrm{~d}_{j \mathrm{k}}-\rho_{\mathrm{n}}{ }^{-1} \theta_{j \mathrm{k}} \mathrm{t}_{j \mathrm{k}}\right)\right)=\operatorname{Diag}\left(\theta_{j \mathrm{k}} \mathrm{r}_{j \mathrm{k}}+\rho^{-1}(1-\rho) \theta_{j \mathrm{k}}{ }^{2} \sigma_{j \mathrm{k}}{ }^{2}\right)
$$

whence follows that the variances. $\theta_{j \mathbf{k}} \mathrm{J}_{j \mathrm{k}}+\rho^{-1}(1-\rho) \theta_{j \mathrm{k}}{ }^{2} \sigma_{i \mathrm{k}}{ }^{2}$ can be consistently estimated by the $v 1_{i \mathrm{i}}$ the empirical 'covariances'. 
Again combining Borgan (18) and Self and Prentice (5) we have also:

$$
\left(n^{1 / 2}\left(\beta_{j k}-\rho_{n}^{-1} \theta_{j k}\right)\right) \rightarrow>_{D} N\left(0,\left(\frac{\rho^{-1} \theta_{j k} I_{j k}+(1-\rho)\left(\rho^{-1} \theta_{j k}\right)^{2} \sigma_{j k}^{2}}{\rho r_{j k}^{2}}\right)\right)
$$

where $\hat{\mu}_{j k}$ is the consistent case-cohort maximum likelihood estimator of $\rho_{\mathrm{n}}{ }^{-1} \theta_{j k}$.

Results like (3) can also be shown for the case-cohort maximum likelihood estimates $\left(\hat{\lambda}_{1}, \ldots, \hat{\lambda}_{\mathrm{J}}, \hat{\psi}_{2}, \ldots, \hat{\psi}_{\mathrm{K}}\right)$ or $\left(\hat{\alpha}_{1}, \ldots, \hat{\alpha}_{\mathrm{j}}, \hat{\beta}_{2}, \ldots, \beta_{\mathrm{K}}\right)$, again using Borgan (18) and Self and Prentice (5). The 'blow-up' approach can be treated analogously and we shall not repeat the results here, see. Volovics (15).

Now, for example, for the case-cohort Mantel-Haenszel estimator $\hat{\psi}$ (in the situation of 2 exposure classes denoted 1 and 2) we have ('shrink' approach):

$$
n^{1 / 2}(\psi-\psi)=\frac{n^{1 / 2} \sum_{j}\left[\frac{\tilde{t}_{j 1}}{\tilde{t}_{j 1}+\tilde{t}_{j 2}}\left(d_{j 2}-\psi \lambda_{j} \tilde{E}_{j 2}\right)-\psi \frac{\tilde{t}_{j 2}}{\tilde{t}_{j 1}+\tilde{t}_{j 2}}\left(d_{j 1}-\lambda_{j} \tilde{t}_{j 1}\right)\right]}{\sum_{j} \frac{\tilde{E}_{j 1} \tilde{E}_{j 2}}{\tilde{t}_{j 1}+\tilde{t}_{j 2}} \cdot \frac{d_{j 1}}{\tilde{E}_{j 1}}}
$$

from which, using the results with respect to the $\left(\mathrm{d}_{j \mathrm{k}}-\theta_{j \mathrm{k}} \mathrm{t}_{j \mathrm{k}}\right)$ above, it can be shown that $n^{1 / h}(\downarrow-\downarrow)$ converges to a normally distributed variable with mean zero and a certain variance which can be estimated by formula (4.2) and that $\hat{\psi}$ is a consistent estimator of *. As the Mantel-Haenszel test (4.3), the two trend tests (4.5) and (4.7) and the heterogeneity chi-square test (4.11) can be expanded likewise, the convergence results with respect to these statistics follow analogously. The 2 trend tests and the chi-square test (4.11) can also be obtained as score tests given a relevant case-cohort likelihood function and then the convergence results and the relevant variance estimator can also be obtained from the pseudolikelihood function results mentioned below. The asymptotics of the two remaining chi-square tests ( 4.9 and 4.10 ) can also be established by expressing these tests as functions of the $\left(d_{j k}-\theta_{j k} t_{j k}\right)$ and following reasoning as in Lehmann (20, chapter 7).

Given pseudolikelihoods like $(5.1 \mathrm{~S})$ or $(5.1 \mathrm{~B})$ where $\lambda_{i}=\exp \left(\hat{\mathrm{x}}_{i}^{\mathrm{T}} \beta\right)$ it is only a slight generalization of the foregoing to show, again combining Borgan (18) and Self and Prentice (5) results that the case-cohort maximum likelihood estimator $\beta$ is a consistent estimator of $\beta$ and that $\mathrm{n}^{1 / 2}(\beta-\beta)$ is asymptotically normally distributed with mean vector 0 and a covariance matrix $\Sigma$ which can be consistently estimated by $C \Delta C$ as specified in 85. The two estimators 5.2 .1 a versus $5.2 .1 \mathrm{~b}$ for example, for the same asymptotic covariance $\Sigma$ can be explained by reasoning like that given above with respect to formulas 1 and 2. The estimator 5.2.1b can also be derived using an estimating function theory approach. Standard asymptotic theory can be used to justify Wald, score, likelihood ratio tests and confidence intervals. 


\title{
Chapter 11
}

\section{A prospective cohort study on dietary fat and the risk of postmenopausal breast cancer*}

Piet A. van den Brandt ${ }^{1}$, Pieter van 't Veer ${ }^{2}$, R. Alexandra Goldbohm ${ }^{1,2}$, Elisabeth Dorant ${ }^{1}$, Alexander Volovics ${ }^{3}$, Rudolph J.J. Hermus ${ }^{2}$, Ferd Sturmans ${ }^{1}$.

1) Department of Epidemiology, University of Limburg, Maastricht, the Netherlands; 2) Department of Nutrition, TNO-Toxicology and'Nutrition Institute, Zeist, the Netherlands; 3) Department of Medical Statistics, University of Limburg, Maastricht, the Netherlands

\begin{abstract}
In 1986 a prospective cohort study on diet and cancer was started In the Netherlands among 62,573 women aged 55-69 years. Baseline information on diet and other risk factors was collected with a questionnaire. Cancer Incidence was measured by record linkage with cancer registries and a pathology register. $\mathbf{A}$ case-cohort approach was used, in which the accumulated persontime in the cohort was estimated by follow-up of a randomly selected subcohort $(n=1,812)$. After 3.3 years of follow-up, 471 Incldent breast cancer cases were avallable for analysis. Questionnalre data of these cases and the 1,716 female subcohort members without a history of cancer other than skin cancer were analyzed. In a multivariate analysis, controlling for traditional risk factors, the relative rates (RR) for breast cancer in increasing quintiles of energy-adjusted total fat intake were: $1.00,1.00,1.34,1.22,1.08$ (p-trend=0.32). For saturated fat there was some evidence for a weak positive association when quintlles were used ( $R \mathbf{R}$ in quintiles 1-5: $1.00,1.22,1.22,1.38,1.39$; p-trend=0.049). The $95 \%$ confinence interval

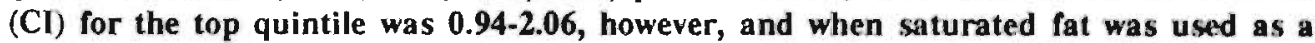
continuous variable, the effect was no longer slgnificant $(p=0.20)$. Relative rate estimates for the highest versus lowest quintlles of monounsaturated fat, polyunsaturated fat and cholesterol intake were $0.75(95 \% \mathrm{Cl}: 0.50-1.12), 0.95(95 \% \mathrm{CI}$ : 0.64-1.40) and 1.09 (95\% CI: 0.74-1.61), respectlvely, with no evidence for significant trends. This prospective study does not support a major role of dletary fat in the etiology of postmenopausal breast cancer.
\end{abstract}




\section{Introduction}

One of the hypotheses on diet and cancer that has attracted considerable attention of investigators and public health officials relates to the role of dietary fat in the development of breast cancer. This is largely based on evidence from ecological studies relating per capita fat consumption (particularly animal fat) to breast cancer incidence or mortality in different countries (1-5) and on early laboratory experiments $(6,7)$. Also, migrant studies have suggested a positive relationship $(8,9)$. Analytic epidemiologic studies in humans have been far less conclusive, however.

Affer an early case control study suggested a positive association between dietary fat and breast cancer incidence (10), subsequent case-control studies have yielded conflicting results regarding the consumption of animal products or various types of fat and their association with breasit cancer risk (11-38). In a recent combined analysis of 12 case-control studies, it was concluded that a significantly positive association existed between breast cancer risk and total, saturated or monounsaturated fat intake in postmenopausal women (39). However, there was considerable heterogeneity between the 12 studies regarding the individual estimates. Although no association was found with fat intake for premenopausal breast cancer in that analysis (39), a recent casecontroll study (28) nevertheless reported only significant associations with fat intake among premenopausal women.

In contrast to the abundance of case-control studies, relatively few prospective cohort studies have been published to date on fat or animal products and breast cancer risk (40.48). Whereas two studies reported a positive association with meat intake $(40,47)$, this was not confirmed in four other studies $(41-43,45)$. The prospective studies which used intake estimates of total fat, various types of fat and energy intake have resulted in a weakly negative $(44)$, weakly positive $(46,48)$ or no significant association (43) with breast cancer risk. With the exception of the studies by Willett et al. (43) and Howe et al. (46), the mentioned cohort studies are limited by the small number of cases and/or the absence of information on habitual intake of energy, total fat and types of fat. Thus, the evidence from cohort studies of sufficient size with a comprehensive assessment of habitual dietary intake is still very scarce. We have conducted a prospective cohort study in the Netherlands among postmenopausal women to evaluate the association between the habitual intake of fat, various types of fat and the incidence of breast cancer.

\section{Materials and methods}

\section{The cohort}

In September 1986, a prospective cohort study on diet and cancer of the breast, colon, rectum, stomach and lung has been initiated in the Netherlands. The cohort included 58,279 men and 62,573 wonnen aged 55-69 years at the start of the study. The study population originated from 204 municipal population registries throughout the country. At baseline, cohort members completed a mailed self-administered questionnaire on dietary habits and other potentiall risk factors for cancer such as smoking habits, job history, education, reproductive history, medical history, and family history of cancer. The study design has been described in detail elsewhere (49). For data processing and analysis the case-cohort approach (50) is used: the cases are enumerated for the entire cohort (numerator information of incidence rates), while the accumulated person years of the entire cohort are estimated using a subcohort sample (providing the denominator information). 
Following this approach, a random subcohort of 3,500 subjects (1688 men, 1812 women) was sampled from the cohort after the baseline exposure measurement. The subcohort has been followed up for vital status information in order to estimate the accumulated persontime in the cohort. This involved personal mailings and (for nonrespondents) additional contacts with municipal population registries, rendering a complete follow-up: after $\mathbf{3 . 3}$ years of follow-up there were no subcohort members lost to follow-up.

\section{Cancer follow-up}

Follow-up for incident cancer consisted of computerized record linkage with all nine regional cancer registries in the Netherlands and with PALGA, the Dutch national data base of pathology reports. The method of record linkage has been published previously (51). Record linkage has been conducted annually with PALGA and the cancer registries. The lag time between diagnosis of cancer and definitive registration in the cancer registries is usually less than three months, but may occasionally extend to 1.5 years. Considering this lag time, the linkage performed in 1991 thus accounted for presumably all cancers diagnosed until the end of 1989. The analysis in this report is restricted to the cancer incidence in the period from September 17, 1986 (cohort baseline measurement) until December 31, 1989, i.e. a follow-up period of 3.3 years.

In this period a total of 1882 cases of breast, colorectal, stomach or lung cancer were detected in the cohort of 120,852 subjects. This observed number of cases was compared with the number of cases that would be expected on the basis of cancer incidence rates, while taking mortality (52) into account. For this purpose, age- and gender-specific cancer incidence rates for breast, colon, rectum, stomach and lung were obtained from the regional cancer registries for the period 1987-89 (for one registry rates of 1983-87 were used). The expected number of cases of the mentioned tumor sites in the 3.3 years of follow-up was estimated at 1971, thus yielding an observed-toexpected ratio of 0.95 , with a $95 \%$ confidence interval of $(0.91,1.00)$ using Byar's approximation (53). Evidence of good coverage of the cohort by the cancer registries and PALGA is also available from another, independent source. Age- and genderspecific data on hospital admissions for cancer (obtained from the National Health Care Information Center) were used to calculate for each municipality the degree of coverage by the cancer registries and PALGA. The results indicated that the mean coverage degree of the municipalities in which the cohort was recruited increased from $98.5 \%$ at the start of the study to $99.5 \%$ at the beginning of 1987 and $100 \%$ at the beginning of 1988 (54). A high degree of coverage was to be expected, because the 204 municipalities participating in the cohort study were purposely selected on the basis of the estimated coverage degree (49).

\section{Population for breast cancer analysis}

Among the 1882 cases, there were 553 female breast cancer cases. After excluding incident cases with in situ carcinoma and women who reported a history of cancer other than skin cancer in the baseline questionnaire, 471 microscopically confirmed incident cases of breast cancer were available for data entry and analysis. After excluding prevalent cancer cases other than skin cancer from the female subcohort of 1812 as well, 1716 subjects remained in this group. The questionnaire data of the subcohort members and of the cancer cases were key-entered twice and processed in a manner blinded with respect to case/subcohort status to avoid bias in coding and interpretation of the data. The evaluation of the associations between breast cancer and traditional, nondietary risk factors was based on the data of the aforementioned 471 cases and 1716 subcohort members. Regarding the dietary part of the questionnaires, about $7 \%$ could 
not be used for nutrient intake calculation because of missing or inconsistent. dietary data (see also next paragraph). The analyses of dietary associations with breast cancer are therefore based on data of 437 breast cancer cases and 1598 subcohort members.

\section{The dietary questionnaire}

Usual diet was assessed at baseline with a semi-quantitative food frequency questionnaire (150 items) specifically designed for, and pretested among men and women of the cohort age range (55). The principal nutrients of interest in the design of the questionnaire were: energy, protein (vegetable, animal), fat (saturated, mono- and polyunsaturated), cholesterol, carbohydrates (mono- and disaccharides, polysaccharides), dietary fiber, alcohol, calcium, vitamin A, B-carotene and vitamin C. Because subjects tended to skip questions on items they did not consume, questionnaires were considered incomplete when either: (a) more than 60 items were left blank and less than 35 items were eaten at least once a month; or (b) one or more item blocks (groupings of items, e.g. beverages) were left blank. More details are given in a separate report (Goldbohm et al., submitted for publication). Mean individual nutrient intakes per day are computed using the Dutch food table of 1986 (56) by cumulating the multiplied frequencies and portion sizes of all food items with their tabulated nutrient contents. The validity of the questionnaire was studied in 1987-88 by comparing it to a nine-day dietary record method among 109 cohort members ( 59 men, 50 women). Pearson correlation coefficients between the dietary record and the questionnaire varied from 0.40 for vitamin $\mathrm{B} 1$ to 0.86 for alcohol intake, with a median of 0.69. After adjustment for energy intake and gender by the residual method (57), correlation coefficients ranged from 0.33 for vitamin B1 to 0.86 for alcohol (median $\mathrm{I}=0.67$ ). Crude and energy-gender-adjusted (in parentheses) correlation coefficients were 0.74 for energy intake, $0.72(0.52)$ for total fat, $0.73(0.58)$ for saturated fat, 0.73 $(0.75)$ for polyunsaturated fat and $0.66(0.62)$ for cholesterol. Correlation coefficients were comparable in men and women.

\section{Data analysis}

Relationships between dietary fat intake and potential confounders for breast cancer were investigated by computing age-- and energy-adjusted (57) intakes of fat and various types of fat among the female subcohort members and comparing the mean intakes in the various categories of the confounders with analysis of variance. Two-sided p-values are used throughout this report.

Although a theoretical approach for analyzing case-cohort studies based on Cox's partial likelihood has been published (58), no standard software was available for computing correct variance estimates for the relative rates and for significance testing. Our analyses are based on the assumption that survival times were exponentially distributed in the current follow-up period, which was confirmed after considering loglog plots of the cumulative survival distribution against the logarithm of the survival time. We developed methods for stratified and multivariate case-cohort analyses, allowing for the additional variance introduced by the subcohort sampling. The analyses were programmed and carried out with the GiLIM statistical package (59) (specific programs are available upon request). Specifically, the analysis methods concern computation of Mantel-Haenszel estimators of the relative rate together with corrected estimates of confidence intervals, Mantel-Haenszel test statistics and tests for trend in the stratified analyses. In the stratified analyses presented in this report, we evaluated the influence of the various risk factors and dietary fat on breast cancer, after stratification on age in three 5-year categories. Fat intake values were adjusted for energy intake by the residual method (57) and categorized as quintiles, according to the distribution in the subcohort. 
In the multivariate analysis, relative rates for quintiles of fat intake were computed, adjusted for other covariates in the model. Confidence intervals for relative rates were computed using corrected variance-covariance estimates. Tests for trend were based on likelihood ratio tests, with scores of 1-5 assigned to the increasing quintiles, as in the stratified analyses.

Analyses were also conducted after excluding cases that occurred in the first year of follow-up. The results were essentially similar; thus only results regarding the complete follow-up period are presented.

\section{Results}

Details on the observed exposure distribution are given in the appendix-table where quintile boundaries and medians within quintiles of daily energy and fat intake are presented for the 1598 female subcohort members with complete dietary questionnaires. The unadjusted median daily intake of total fat ranged from $47.0 \mathrm{~g}$ in the lowest quintile to $104.8 \mathrm{~g}$ in the highest quintile. After adjustment for energy intake these medians were 61.0 and $85.5 \mathrm{~g}$ /day, respectively. Likewise, a reduction of variation in intake estimates of the other fats and cholesterol occurred after adjustment for energy intake. In this population energy intake was strongly associated with various fats; the Pearson correlation coefficients between energy and fat intake were 0.89 for total fat, 0.81 for saturated fat, 0.84 for monounsaturated fat, 0.59 for polyunsaturated fat and 0.59 for cholesterol intake, respectively. Expressed as percentage of energy intake, the median energy contribution of total fat ranged from $32.1 \%$ in the lowest quintile to $46.2 \%$ in the highest quintile. For saturated fat, these values were $12.1 \%$ and $19.9 \%$, respectively.

Table 1 shows the age-adjusted mean intake of energy and the mean intake of fats, adjusted for age and energy intake, among the 1598 subcohort members. The average mean daily intake was $1689 \pm 409 \mathrm{kcal}$ for energy (mean \pm SD), $74.2 \pm 10.5 \mathrm{~g}$ for total fat, $29.7 \pm 5.7 \mathrm{~g}$ for saturated fat, $27.6 \pm 5.2 \mathrm{~g}$ for monounsaturated fat, $15.3 \pm 6.2 \mathrm{~g}$ for polyunsaturated fat and $235 \pm 60 \mathrm{mg}$ for cholesterol. The table also shows the mean intake values according to the categories of various traditional nondietary confounders. Energy intake was significantly positively associated with age at first birth $(p<0.05)$, age at menopause $(p<0.01)$ and use of oral contraceptives $(p<0.05)$. Significantly negative associations existed between energy intake, an artificially induced menopause $(p<0.01)$ and Quetelet index $(\mathrm{p}<0.01)$. Total fat and monounsaturated fat intake were significantly associated with a history of benign breast disease $(p<0.05)$. Whereas saturated fat intake showed no significant associations with any of the other risk factors, both monounsaturated and polyunsaturated fat intake were significantly inversely associated with highest attained level of education $(p<0.05)$. Cholesterol intake was significantly associated with age at first birth $(p<0.05)$, parity $(p<0.05)$ and Quetelet index $(p<0.001)$. Overall, the differences in intake between the various categories of the mentioned confounding factors were relatively small.

In table 2 the results. of the stratified analyses for nondietary established and potential risk factors for breast cancer are shown, after stratification by age in 5-year categories. In this table the observed person years in the subcohort are shown, together with the number of incident cases in each stratum. To illustrate the principle of the case-cohort approach, the breast cancer incidence rate in the cohort can be estimated after inflating the person years in the subcohort with the inverse of the subcohort sampling fraction, i.e., 62573/1812. For example, the estimated crude breast cancer incidence rate in women without a history of benign breast disease would be $411 /(5150 \times 62573 / 1812)$ or 231 per 100,000 person years. 
Table 1. Mean daily intake of energy (age-adjusted) and fats (age- and energy-adjusted), according to various characteristics, in female subcohort members with complete dietary data $(\mathrm{n}=1598)$.

\begin{tabular}{|c|c|c|c|c|c|c|c|}
\hline \multirow{3}{*}{ Characteristic } & \multirow{3}{*}{$\mathbf{n}^{*}$} & \multicolumn{6}{|c|}{ Mean daily intake } \\
\hline & & \multirow{2}{*}{$\frac{\text { Age-adj. }}{\text { Energy }}$} & \multicolumn{5}{|c|}{ Age- and energy-adjusted } \\
\hline & & & $\begin{array}{l}\text { Total } \\
\text { fat (g) }\end{array}$ & $\begin{array}{l}\text { Saturated } \\
\text { fat }(\mathrm{g})\end{array}$ & $\begin{array}{l}\text { Mono-unsat. } \\
\text { fat }(\mathrm{g})\end{array}$ & $\begin{array}{l}\text { Poly-unsat. } \\
\text { fat }(\mathrm{g})\end{array}$ & $\begin{array}{l}\text { Cholesterol } \\
\text { (mg) }\end{array}$ \\
\hline Total & 1598 & 1689 & 74.2 & 29.7 & 27.6 & 15.3 & 235 \\
\hline $\begin{array}{l}\text { Benign breast disease } \\
\text { No } \\
\text { Yes }\end{array}$ & $\begin{array}{r}1476 \\
122\end{array}$ & $\begin{array}{l}1690 \\
1672\end{array}$ & $\begin{array}{r}\dagger \\
74.4 \\
72.2\end{array}$ & $\begin{array}{l}29.8 \\
28.9\end{array}$ & $\begin{array}{r}\dagger \\
27.7 \\
26.6\end{array}$ & $\begin{array}{l}15.3 \\
15.2\end{array}$ & $\begin{array}{l}236 \\
231\end{array}$ \\
\hline $\begin{array}{l}\text { Maternal breastca } \\
\text { No } \\
\text { Yes }\end{array}$ & $\begin{array}{r}1548 \\
50\end{array}$ & $\begin{array}{l}1691 \\
1611\end{array}$ & $\begin{array}{l}74.2 \\
74.3\end{array}$ & $\begin{array}{l}29.7 \\
30.0\end{array}$ & $\begin{array}{l}27.6 \\
27.8\end{array}$ & $\begin{array}{l}15.3 \\
14.9\end{array}$ & $\begin{array}{l}235 \\
230\end{array}$ \\
\hline $\begin{array}{l}\text { Breastca in sister(s) } \\
\text { No } \\
\text { Yes }\end{array}$ & $\begin{array}{r}1515 \\
83\end{array}$ & $\begin{array}{l}1687 \\
1719\end{array}$ & $\begin{array}{l}74.2 \\
74.5\end{array}$ & $\begin{array}{l}29.8 \\
29.5\end{array}$ & $\begin{array}{l}27.6 \\
27.9\end{array}$ & $\begin{array}{l}15.2 \\
15.5\end{array}$ & $\begin{array}{l}236 \\
223\end{array}$ \\
\hline $\begin{array}{l}\text { Parity } \\
\text { Nulliparous } \\
\qquad \begin{aligned} 1 \\
2 \\
\geq 3\end{aligned}\end{array}$ & $\begin{array}{l}275 \\
129 \\
347 \\
823\end{array}$ & $\begin{array}{l}1697 \\
1702 \\
1658 \\
1699\end{array}$ & $\begin{array}{l}74.8 \\
74.5 \\
73.8 \\
74.1\end{array}$ & $\begin{array}{l}30.2 \\
29.7 \\
29.9 \\
29.5\end{array}$ & $\begin{array}{l}27.3 \\
27.4 \\
27.5 \\
27.8\end{array}$ & $\begin{array}{l}15.5 \\
16.0 \\
14.8 \\
15.2\end{array}$ & $\begin{array}{r}\dagger \\
227 \\
231 \\
239 \\
237\end{array}$ \\
\hline $\begin{array}{l}\text { Age at first birth (yrs) } \\
17-19 \\
20-24 \\
25-29 \\
\geq 30 \\
\text { Nulliparous }\end{array}$ & $\begin{array}{r}23 \\
342 \\
653 \\
292 \\
275\end{array}$ & $\begin{array}{l}+ \\
1416 \\
1673 \\
1699 \\
1707 \\
1697\end{array}$ & $\begin{array}{l}73.3 \\
74.2 \\
74.3 \\
73.7 \\
74.8\end{array}$ & $\begin{array}{l}29.3 \\
291.7 \\
29.7 \\
29.5 \\
30.2\end{array}$ & $\begin{array}{l}26.8 \\
27.7 \\
27.8 \\
27.6 \\
27.3\end{array}$ & $\begin{array}{l}15.3 \\
15.3 \\
15.2 \\
15.1 \\
15.5\end{array}$ & $\begin{array}{r}\dagger \\
242 \\
239 \\
239 \\
230 \\
227\end{array}$ \\
\hline $\begin{array}{l}\text { Age at menarche (yrs) } \\
\leq 12 \\
\quad 13 \\
\geq 14\end{array}$ & $\begin{array}{l}401 \\
736 \\
442\end{array}$ & $\begin{array}{l}1671 \\
1689 \\
1709\end{array}$ & $\begin{array}{l}74.2 \\
74.0 \\
74.5\end{array}$ & $\begin{array}{l}29.8 \\
291.7 \\
29.6\end{array}$ & $\begin{array}{l}27.4 \\
27.5 \\
28.0\end{array}$ & $\begin{array}{l}15.4 \\
15.1 \\
15.4\end{array}$ & $\begin{array}{l}239 \\
233 \\
236\end{array}$ \\
\hline $\begin{array}{l}\text { Age at menopause (yrs) } \\
\leq 44 \\
45-49 \\
50-54 \\
\geq 55\end{array}$ & $\begin{array}{l}232 \\
479 \\
673 \\
117\end{array}$ & $\begin{array}{l}\ddagger \\
1609 \\
1676 \\
1724 \\
1730\end{array}$ & $\begin{array}{l}75.0 \\
74.4 \\
73.6 \\
74.2\end{array}$ & $\begin{array}{l}30.0 \\
29.6 \\
29.8 \\
29.7\end{array}$ & $\begin{array}{l}28.1 \\
27.7 \\
27.4 \\
27.4\end{array}$ & $\begin{array}{l}15.3 \\
15.6 \\
14.8 \\
15.3\end{array}$ & $\begin{array}{l}240 \\
231 \\
234 \\
251\end{array}$ \\
\hline $\begin{array}{l}\text { Artificial menopause } \\
\text { No } \\
\text { Kes }\end{array}$ & $\begin{array}{r}1304 \\
251\end{array}$ & $\begin{array}{r}\ddagger \\
1702 \\
1621\end{array}$ & $\begin{array}{l}74.1 \\
74.8\end{array}$ & $\begin{array}{l}29.7 \\
29.8\end{array}$ & $\begin{array}{l}27.5 \\
28.2\end{array}$ & $\begin{array}{l}15.2 \\
15.2\end{array}$ & $\begin{array}{l}234 \\
240\end{array}$ \\
\hline $\begin{array}{l}\text { Use of oral contraceptives } \\
\text { Never } \\
\text { Ever }\end{array}$ & $\begin{array}{r}1180 \\
394\end{array}$ & $\begin{array}{l}+1 \\
1676 \\
1735\end{array}$ & $\begin{array}{l}74.3 \\
74.0\end{array}$ & $\begin{array}{l}29.8 \\
29.6\end{array}$ & $\begin{array}{l}27.6 \\
27.6\end{array}$ & $\begin{array}{l}15.2 \\
15.3\end{array}$ & $\begin{array}{l}234 \\
240\end{array}$ \\
\hline $\begin{array}{l}\text { Highesi level of education } \\
\text { Low } \\
\text { Medium } \\
\text { Hight }\end{array}$ & $\begin{array}{l}902 \\
550 \\
135\end{array}$ & $\begin{array}{l}1675 \\
1704 \\
1721\end{array}$ & $\begin{array}{l}74.5 \\
74.2 \\
72.5\end{array}$ & $\begin{array}{l}29.6 \\
29.9 \\
30.1\end{array}$ & $\begin{array}{r}+ \\
27.9 \\
27.3 \\
26.9\end{array}$ & $\begin{array}{r}\dagger \\
15.4 \\
15.3 \\
13.8\end{array}$ & $\begin{array}{l}234 \\
235 \\
240\end{array}$ \\
\hline $\begin{array}{l}\text { Curreni cigarette smoking } \\
\text { No } \\
\text { Yes }\end{array}$ & $\begin{array}{r}1266 \\
332\end{array}$ & $\begin{array}{l}1694 \\
1669\end{array}$ & $\begin{array}{l}74.3 \\
73.9\end{array}$ & $\begin{array}{l}29.8 \\
29.6\end{array}$ & $\begin{array}{l}27.6 \\
27.7\end{array}$ & $\begin{array}{l}15.3 \\
15.0\end{array}$ & $\begin{array}{l}235 \\
237\end{array}$ \\
\hline $\begin{array}{l}\text { Ouetelet index }\left(\mathrm{kg} / \mathrm{m}^{2}\right) \\
\leq 22 \\
23.24 \\
25.26 \\
\geq 27\end{array}$ & $\begin{array}{l}412 \\
436 \\
308 \\
395\end{array}$ & $\begin{array}{l}\ddagger \\
1724 \\
1723 \\
1656 \\
1630\end{array}$ & $\begin{array}{l}73.7 \\
74.5 \\
74.1 \\
74.8\end{array}$ & $\begin{array}{l}29.9 \\
29.8 \\
29.4 \\
29.8\end{array}$ & $\begin{array}{l}27.3 \\
27.6 \\
27.5 \\
28.2\end{array}$ & $\begin{array}{l}14.8 \\
15.4 \\
15.6 \\
15.3\end{array}$ & $\begin{array}{r}8 \\
227 \\
232 \\
232 \\
251\end{array}$ \\
\hline
\end{tabular}

- Due to missing questionnaire data on non-dietary factors, numbers may not add up to 1598 subjects

$\dagger$ p-value (Analysis of variance comparing means) $<0.05 ; \ddagger$-value $<0.01 ; \S \mathrm{p}$-value $<0.001$ 
Table 2. Mantel-Haensel relative rate of breast cancer according to various characteristics, stratified by age ( 3 categories).

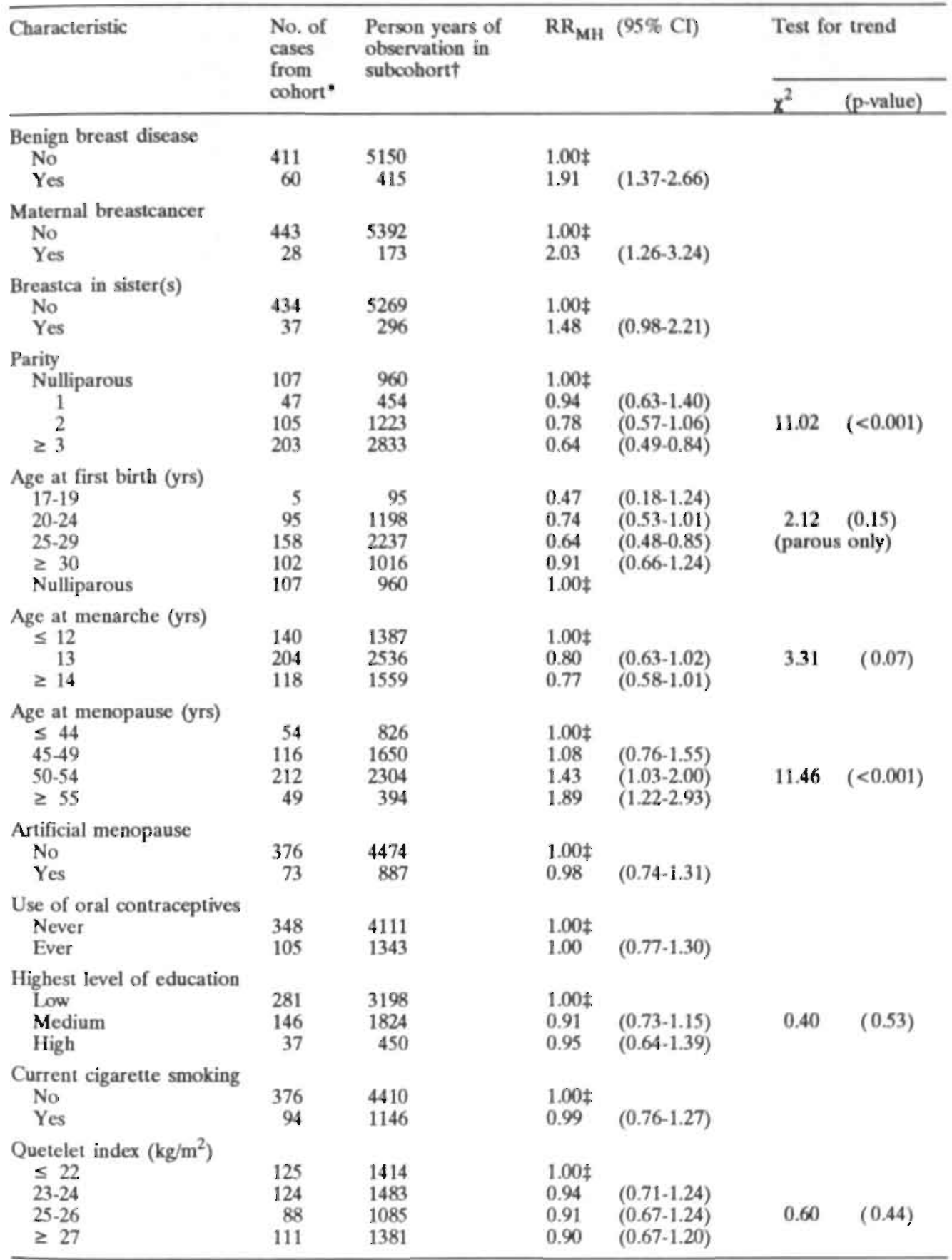

- Due to missing questionnaire data, the number of cases may be less than 471 .

$\dagger$ The number of person years in the total cohort can be estimated by multiplying the subcohort person years by $62573 / 1812$ (i.e., the inverse of the sampling fraction).

† Reference category. 
As can be seen from table 2, the effects of the established risk factors are in the anticipated direction. Elevated risks were found for women with a history of benign breast disease (Mantel-Haenszel relative rate, $\mathrm{RR}=1.91 ; \mathrm{p}<0.001$ ), history of breast cancer in mother $(\mathrm{RR}=2.03 ; \mathrm{p}=0.01)$ and history of breast cancer among one or more sisters $(\mathrm{R} R=1.48 ; \mathrm{p}=0.08$ ). Age at first birth was positively associated with the risk of breast cancer, although the test for trend among parous women only was not significant $(\mathrm{p}=0.15)$. When nulliparous were included, the test for trend became highly significant $\left(\chi^{2}=8.38, p=0.004\right)$. Parity showed a significantly negative association with breast cancer risk (test for trend: $\mathrm{p}<0.001$ ). Age at menarche was negatively associated with breast cancer risk, although not significantly ( $\mathrm{p}$-trend $=0.07$ ), whereas age at menopause was significantly associated with an elevated risk of breast cancer $(p<0.001)$. No significant associations were observed with artificial menopause (induced by hormones or surgical) ( $R R=0.99 ; p=0.93)$, use of oral contraceptives $(R R=1.00 ; p=0.99$ ), level of education ( $p$-trend $=0.53$ ), current cigarette smoking ( $R=0.99 ; p=0.91)$ or Quetelet index ( $\mathrm{p}$-trend $=0.44)$.

Table 3 shows the observed Mantel-Haenszel relative rates for breast cancer according to quintiles of energy and energy-adjusted fat intake, after stratification by age. No significant associations were found with energy intake or energy-adjusted intake of total fat, saturated fat, monounsaturated or polyunsaturated fat nor cholesterol. Whereas the relative rate estimates, for saturated fat were above the null-value, those for monounsaturated fat and polyunsaturated fat were generally somewhat below the null-value. None of the tests for trend was significant, however. With regard to energy intake, thta' fat and sholesteol, essentially no asseciation was sbserved. The same picture emerged for fat intake quintiles that were not adjusted for energy intake or when fat intake was expressed as percentage energy contribution (results not shown).

The associations between the risk of breast cancer and fat intake were further evaluated in a multivariate model with adjustment for age, history of benign breast disease, maternal breast cancer, breast cancer in sister(s), age at menarche, age at menopause, oral contraceptive use, parity, age at first birth, Quetelet index, education, habitual alcohol use and current cigarette smoking. The results are presented in table 4 . As anticipated from the earlier associations in table 1 , this adjustment did not alter the relative rate estimates appreciably. For total fat, the relative rate of breast cancer increased to 1.34 and 1.29 in the third and fourth quintile of intake, respectively, but decreased to 1.08 in the fifth quintile. The test for trend was not significant, nor were any of the quintile-specific relative rates significantly different from unity. When energyadjusted total fat intake was entered as a continuous variable in the model, the relative rate associated with an increase of $25.5 \mathrm{~g} /$ day (i.e., the difference in energy-adjusted median intake between the fifth and first quintile) was 1.08 (95\% CI: $0.82-1.44)$. For saturated fat, a positive association with the risk of breast cancer was observed in the multivariate analysis, with relative rate estimates of $1.22,1.22,1.38$ and 1.39 for the second to fifth quintile, respectively ( $\mathrm{p}$-trend $=0.049$ ). None of quintile estimates was significantly different from the null value, however. When saturated fat was entered as a continuous variable in the model, no significant association was observed $(p=0.20)$. The relative rate of breast cancer for an increased intake of $14.3 \mathrm{~g}$ saturated fat per day (difference in medians between fifth and first quintile) was 1.18 (95\% CI: 0.89-1.59). 
Table 3. Mantel-Haenszel relative rate of breast cancer according to quintiles of energy intake and of energy-adjusted fat intake, stratified by age ( 3 categories).

\begin{tabular}{|c|c|c|c|c|c|c|}
\hline \multirow[t]{2}{*}{ Characteristic } & \multirow{2}{*}{$\begin{array}{l}\text { No. of } \\
\text { cases } \\
\text { from } \\
\text { cohort" }\end{array}$} & \multirow{2}{*}{$\begin{array}{l}\text { Person years of } \\
\text { observation in } \\
\text { subcohort }\end{array}$} & \multirow[t]{2}{*}{$\mathrm{RR}_{\mathrm{MH}}$} & \multirow[t]{2}{*}{$(95 \% \mathrm{CI})$} & \multicolumn{2}{|c|}{ Test for trend } \\
\hline & & & & & $x^{2}$ & (p-value) \\
\hline \multicolumn{7}{|c|}{ Energy (quintiles) } \\
\hline 1 (low) & 83 & 1032 & $1.00 \dagger$ & & \multirow{5}{*}{0.06} & \multirow{5}{*}{$(0.81)$} \\
\hline 2 & 83 & 1035 & 0.99 & $(0.71-1.40)$ & & \\
\hline 3 & 98 & 1046 & 1.17 & $(0.84-1.63)$ & & \\
\hline 4 & 92 & 1040 & 1.11 & $(0.79-1.55)$ & & \\
\hline 5 (high) & 81 & 1034 & 0.99 & $(0.70-1.39)$ & & \\
\hline \multicolumn{7}{|l|}{ Total fat } \\
\hline 1 & 88 & 1038 & $1.00 \dagger$ & & \multirow{5}{*}{0.10} & \multirow{5}{*}{$(0.75)$} \\
\hline 2. & 75 & 1034 & 0.84 & $(0.59-1.18)$ & & \\
\hline 3 & 95 & 1036 & 1.08 & $(0.78-1.51)$ & & \\
\hline 4 & 95 & 1040 & 1.07 & $(0.77-1.49)$ & & \\
\hline 5 & 84 & 1040 & 0.95 & $(0.68-1.34)$ & & \\
\hline \multicolumn{7}{|l|}{ Saturated fat } \\
\hline 1 & 76 & 1039 & $1.00 t$ & & \multirow{5}{*}{2.91} & \multirow{5}{*}{$(0,09)$} \\
\hline 2 & 84 & 1036 & 1.14 & $(0.80-1.62)$ & & \\
\hline 3 & 81 & 1042 & 1.07 & $(0.75-1.52)$ & & \\
\hline 4 & 97 & 1037 & 1.32 & $(0.93-1.86)$ & & \\
\hline 5 & 99 & 1033 & 1.29 & $(0.92-1.81)$ & & \\
\hline \multicolumn{7}{|c|}{ Monounsaturated fat } \\
\hline 1 & 95 & 1034 & $1.00 \dagger$ & & \multirow{5}{*}{3.00} & \multirow{5}{*}{$(0.08)$} \\
\hline 2 & 91 & 1042 & 0.94 & $(0.68-1.31)$ & & \\
\hline 3 & 98 & 1036 & 1.05 & $(0.76-1.45)$ & & \\
\hline 4 & 82 & 1035 & 0.85 & (0.61-1.19), & & \\
\hline 5 & 71 & 1040 & 0.75 & $(0.53-1.05)$ & & \\
\hline \multicolumn{7}{|c|}{ Polyunsaturated fat } \\
\hline 1 & 97 & 1031 & $1.00 \dagger$ & & \multirow{5}{*}{0.47} & \multirow{5}{*}{$(0.49)$} \\
\hline 2 & 83 & 1041 & 0.84 & $(0.60-1.18)$ & & \\
\hline 3 & 89 & 1040 & 0.92 & $(0.66-1.28)$ & & \\
\hline 4 & 84 & 1033 & 0.87 & $(0.62-1.22)$ & & \\
\hline 5 & 84 & 1042 & 0.85 & $(0.61-1.19)$ & & \\
\hline \multicolumn{7}{|l|}{ Cholesterol } \\
\hline 1 & 90 & 1037 & $1.00 \dagger$ & & \multirow{5}{*}{0.00} & \multirow{5}{*}{$(0.99)$} \\
\hline 2 & 88 & 1042 & 0.98 & $(0.70-1.37)$ & & \\
\hline 3 & 84 & 1040 & 0.91 & (0.65-1.28) & & \\
\hline 4 & 80 & 1032 & 0.87 & $(0.62-1.23)$ & & \\
\hline 5 & 95 & 1036 & 1.04 & $(0.75-1.44)$ & & \\
\hline
\end{tabular}

- There were 437 cases with complete dietary data.

$\dagger$ Reference category. 
Table 4. Relative rate of breast cancer according to quintiles of energy-adjusted fat intake in multivariate analysis.

Nutrient

RR* (95\% CI)

Test for trend

$x^{2}$

(p-value)

Total fat, quintiles

$\begin{array}{lll}1 \text { (low) } & 1.00 \dagger & \\ 2 & 1.00 & (0.67-1.49) \\ 3 & 1.34 & (0.91-1.97) \\ 4 & 1.29 & (0.88-1.91 \\ 5 \text { (high) } & 1.08 & (0.73-1.59\end{array}$

aturated fat

\section{1}

2

3

4

5

Monounsaturated fat

1
2
3
4
5

Polyunsaturated fat

$\begin{array}{ll}1 & 1.00+ \\ 2 & 0.91 \\ 3 & 0.90 \\ 4 & 1.09 \\ 5 & 0.95\end{array}$

Cholesterol

$\begin{array}{lll}1 & 1.00 \dagger & \\ 2 & 0.84 & (0.57-1.24) \\ 3 & 0.85 & (0.57-1.26) \\ 4 & 0.85 & (0.57-1.27) \\ 5 & 1.09 & (0.74-1.61)\end{array}$

(0.81-1.84)

(0.82-1.84)

(0.92-2.06)

(0.94-2.06)

3.87

(0.049)

(0.71-1.51)

$(0.69-1.46)$

$(0.67-1.46)$

(0.50-1.12)

2.33

0.99

0.75

(0.62-1.34)

(0.61-1.32)

0.04

(0.85)

(0.64-1.40)

$(0.74-1.61)$

- Relative rate after adjustment for: age, history of benign breasi disease, maternal breast cancer, breast cancer in sister(s), age at menarche, age at menopause, oral contraceptive use, parity, age at first birth, Quetelet index, education, alcohol use, current cigarette smoking.

† Reference category.

Monounsaturated fat intake was not associated with the risk of breast cancer in the multivariate analysis; the relative rates for increasing quintiles were $1.00,1.03,1.00,0.99$ and $0.75(p$-trend $=0.13)$. Polyunsaturated fat and cholesterol intake also did not show any association with breast cancer risk; the relative rates for the highest quintile compared to the lowest were 0.95 and 1.09 , respectively. When fat intake was expressed as percentage contribution to energy intake, no significant trends were observed in the multivariate analyses. For example, for total fat the relative rates in increasing quintiles of consumption were $1.00,1.09,1.51,1.22$ and 1.02 ( $p$-trend=0.734), with the medians 
of these quintiles corresponding to $32.1,36.6,39.3,42.1$ and 46.2 energy-\%, respectively. For saturated fat the relative rates were estimated as $1.00,0.98,0.99,1.21$ and 1.24 (p-trend=0.093). In addition, when a fat decomposition model was used $(60)$, again no significant effect of saturated fat intake was noted (results of latter analyses not shown).

\section{Discussion}

We found no evidence for an elevated risk of breast cancer with increasing intake of total fat, when adjusted for energy intake. For saturated fat, a weak positive trend with risk of breast cancer was observed, with increases in relative rate up to 1.39 for those in the highest intake quintile. However, none of the relative rates in the higher quintiles of saturated fat intake was significantly different from unity. An association would have been more likely if the positive trend was also significant when saturated fat was entered as a continuous variable. This, however, was not the case. For polyunsaturated fat and cholesterol intake, essentially no evidence for a relationship was found. Monounsaturated fat intake was, if anything, negatively associated with breast cancer risk, but not significantly.

Before discussing these results in relation to other studies on dietary fat and breast cancer, we will first describe the strengths and limitations of this study. These refei to the source of the population and the selected age range, homogeneity of dietary fat intake, misclassification of exposure, selection and confounding bias and the length of follow-up. This study was carried out in a large sample of the general population of women aged 55-69 years at baseline, which yields a sufficient number of cases to study etiologic relationships (61). A potential disadvantage of this approach is that potential effects of diet early in life cannot be studied. However, most laboratory experiments have suggested an effect of dietary fat on the promotional phase of breast cancer (e.g., $62)$, and recent case-control studies $(25,32)$ and a cohort study $(45)$ indicated no strong associations between fat intake during childhood or adolescence and breast cancer risk. Also, most epidemiologic studies on diet and breast cancer with positive results indicate an effect on postmenopausal rather than premenopausal breast cancer (39).

Although the Netherlands is known for its high per capita fat intake (63), etiologic studies might be hampered when the population is relatively homogeneous regarding dlietary fat intake. In our cohort, the unadjusted total fat intake varied from a median of $47 \mathrm{~g} /$ day (or, $32 \%$ of totall energy) in the lowest quintile to $105 \mathrm{~g} /$ day (46 energy- $\%$ ) in the highest quiintile according to our questionnaire data. The questionnaire data may have overestimated the variability that actually existed in our cohort. In this respect, the medians of the lowest and highest quintiles of intake according to the 9-day jecord method in our validation study were 34 and 46 energy-\%, respectively. It may be that fat intake needs to be reduced more substantially to exert an effect, although a true reduction of habitual fat intake below 30 energy-\% may prove to be very difficult to achieve in free-living Western populations. Also, epidemiological studies that included subjects with observed fat intakes as low as $20 \%$ of total energy or less $(21,27)$ have produced negative findings with respect to breast cancer risk. Results from studies. among southern-European populations in Greece and Italy with presumably more hetterogeneity in the diet than the Netherlands were inconsistent $(13,23,33)$.

An issue that is related to the observed range of exposure is the misclassification of exposure that is inherent in dietary epidemiological studies. For our study we used a. semiquantitative food frequency questionnaire, which was designed to capture the etiologically interesting variation in intake between individuals. Our validation study indicated a reasonably good validity, which is partly explained by the large number of 
included food items. Most dietary assessment methods in the diet and breast cancer studies were not validated and included considerably less items, sometimes as few as 10 or less (e.g., 31,33,36,40). This also limits the possibility to adjust for energy intake, which is of importance considering the often strong correlation between fat and energy intake (64) and the recent laboratory evidence suggesting that energy intake rather than fat may be implicated as a determinant of breast cancer (65-67).

Selection bias due to loss to follow-up is tunlikely in our study considering the $100 \%$ complete follow-up of person years and the high completeness of cancer follow-up. Also, there are no reasons to assume that there is insufficient control of confounding in our analysis. We measured and controlled for the established risk factors of breast cancer. Moreover, the relative risk estimates were not materially affected by controlling for these factors because of the weak associations with fat intake.

A potential drawback of the study is the stili limited length of follow-up, i.e. 3.3 years. When we excluded cases occurring during the first year of follow-up, the conclusions about the effect of fat were not different irom those based on the total group of cases. Although it cannot be excluded that a stronger effect of fat will become apparent when a longer follow-up period will be available, other cohort studies employing longer follow-up periods do not provide a clear indication for this possibility. Knekt et al. (46) observed a borderline significantly positive association with monounsaturated fat after 20 years of follow-up, but in another cohort study with 21 years of follow-up no significantly elevated risks were found (42). Other cohort studies with five to ten years of follow-ip mostly produced no significant associations with fat or meat intake $(40,44,45,48,68)$. In future analyses we will evaluate whether the estimated association between dietary fat and breast cancer will change with a longer follow-up period.

A review of the published case-control studies on dietary fat and breast cancer reveals that the results vary substantially. Of the case-control studies that measured fat intake, eight showed a significant positive association between intake of total fat or a particular type of fat and breast cancer risk (10-17). In 12 other case-control studies no (significant positive) association was reported (18-29). In a number of case-control studies only the intake of some specific fat-containing foods such as meat or dairy produce was measured, but the results were also inconsistent. Significant positive associations were found with meat intake in six studies $(18,26,28,31-33)$ with intake of dairy produce in five studies $(13,18,31,34,35)$, whereas no (significant positive) associations with meat intake were detected in seven studies $(13,16,34-38)$ and with dairy produce in two studies $(32,38)$.

In prospective cohort studies, the potential problem of biased recall of past food intake (inherent in case-control studies) is avoided. Hirayama found a positive relationship with meat consumption in Japan, but there were only 14 cases in the exposed group (daily meat consumption) and no significance testing was described (40). The cohort studies among US nurses (64) and among special exposure groups such as Seventh Day Adventists $(42,45)$ and nuns eating little or no meat (41) were negative with regard to meat and breast cancer risk. Vatten et al. (47) recently reported an increased risk associated with the consumption of meat at hot meals. However, there was no control in the analysis for various reproductive factors, nor for energy intake. We did also analyze the relationship between meat intake and breast cancer in our cohort study and found the rate ratio of breast cancer for women consuming meat $0-1$ days/week relative to those consuming meat $5-7$ days/week to be 1.23 (95\% CI: 0.63 2.37), with no evidence for a trend. Only four prospective cohort studies have used dietary assessment methods that permitted calculation of energy intake $(43,44,46,48)$. Jones et al. (44) found significantly negative associations between total and saturated fat intake and breast cancer risk, but this was based on 24-hr dietary recall data which 
may not adequately represent an individuals' habitual intake. Knekt et al. (46) reported positive associations with energy-adjusted intake of fats but the trend was only significant for monounsaturated fat. Even after a follow-up of 20 years, the number of incident cases was small, however. Willett et al. (43) and Howe et al. (48) have reported results from large cohorts of women in the US and Canada, respectively. Willett et al. observed no effects of high fat or cholesterol intake on breast cancer risk after four (43) and eight years of follow-up (68). Howe et al. reported slightly elevated relative risks of 1.2-1.3 in the highest quartile of intake for total fat and monounsaturated fat, with marginally significant tests for trend (48). However, the relative risk estimates in highest category were not significantly different from unity, similarly to what we observed in our study regarding saturated fat.

In conclusion, we found no significant association between total fat intake and postmenopausal breast cancer risk. There was some evidence for a weak positive relationship with saturated fat intake but not with the other types of fat or cholesterol. The significance of the association with saturated fat depended on the model specification and was inconsistent. We cannot exclude that a longer follow-up period may yield positive findings, but the current study does not indicate that fat intake is strongly related to breast cancer risk. If dietary fat is etiologically relevant, our study suggests it is accountable to saturated fat.

\section{References}

1. Armstrong B, Doll R. Environmental factors and cancer incidence and morality in different countries, with special reference to dietary practices. Int J Cancer 1975; 15: 617-631.

2. Carroli KK. Experimental evidence of dietary factors and hormone-dependent cancers. Cancer Res 1975; $35: \mathbf{3 3 7 4 1 - 3 3 8 3 .}$

3. Drasar BS, Irving D. Environmental factors and cancer of the colon and breast. Br J Cancer 1973; 27: $167-172$.

4. Gray GE, Pike MC, Henderson BE. Breast cancer incidence and mortality rates in different countries in relation to known risk: factors and dietary praclices. Br $\mathbf{J}$ Cancer 1979; 39: 1-7.

5. Prentice RI, Sheppard L. Dietary fat and cancer: consistency of the epidemiologic data, and disease prevention that may follow from a practical reduction in fat consumption. Cancer Causes Control $1990 ; 1: 81-97$.

6. Tannenbaum A. Genesis and growth of tumours. III. Effect of a high-fat diet. Cancer Res 1942; 2: 468-475.

7. Carroll K.K. Khor HT. Effecis of level and type of dietary fat on incidence of mammary tumours induced in female Sprague-Dawley rats by 7,12-dimethylbenz(a)anthracene. Lipids $1971 ; 6: 415-420$.

8. Haenszei W, Kurihara M. Studies of Japanese migrants. I. Mortality from cancer and other diseases among Japanese in the United States, J Natl Cancer Inst 1968; 40: 43-68.

9. Buell P. Changing incidence of breast canceer in Japanese-Ameriçan women. J Natl Cancer Inst 1973 ; $51: 1479-1483$.

10. Miller AB, Kelly A, Choi NW, Matthews V, Morgan RW, Munan I, Burch JD, Feather J, Howe GR, Jain M. A study of diet and breast cancer. Am J Epidemiol 1978; 107: 499-509.

1. Sarin R, Tandon RK, Paul S. Diet, body fat and plasma lipids in breast cancer. Indian Ji Med Res $1985 ; 81: 493-498$.

12. Lubin F, Wax: $\mathbf{Y}_{n}$ Modan B. Role of fat, animal protein, and dietary fiber in breast cancer etiology: a case-control study. J Natl Cancer Inst 1986; 77: 605-612.

13. Toniolo P, Riboli $\mathrm{F}_{\mathrm{n}}$, Protta F, Charrel M, Cappa APM. Calorie-providing nutrients and risk of breast cancer. J Natl Cancer Inst 1989; 81: 278-286.

14. Yu SZ, Lu RF, Xu DD, Howe GR. A case-control study of dietary and nondietary risk factors for breast cancer in Shanghai. Cancer Res 1990; 50: 5017-5021.

15. Van 't Veer P, Kok FJ, Brants HAM, Ockhuizen T, Sturmans F, Hermus RJ. Dietary fat and the risk of breast cancer. Int J Epidemiol 1990; 19: 12-18.

16. Ewertz M, Gill C. Dietary factors and breast-cancer risk in Denmark. Int J Cancer 1990; 46: $779-784$. 
17. Richardson $\mathrm{S}$, Gerber $\mathrm{M}$, Cenee $\mathrm{S}$. The role of fat, animal protein and some vitamin consumption in breast cancer: a case control study in southern France. Int I Cancer 1991; 48: 1-9.

18. Nomura A, Henderson BE, Lee J. Breast cancer and diet among the Japanese in Hawaii. Am J Clin Nutr 1978; 31: 2020-2025.

19. Graham S, Marshall J. Mettlin C, Rzepka T, Nemoto T, Byers T. Diet in the epidemiology of breast cancer. Am J Epidemiol 1982; 116: 68-75.

20. Kolonel IN, Nomura AMY, Hinds MW, Hirohata T, Hankin JH. Role of diet in cancer incidence in Jlawaii. Cancer Res 1983; 43: 2397s-2403s.

21. Hirohata T, Shigematsu T, Nomura AM, Nomura Y, Horie A, Hirohata I. Occurrence of breast cancer in relation to diet and reproductive history: a case-control study in Fukuoka, Japan. Natl Cancer Inst Monogr 1985; 69: 187-190.

22. Hirohata T, Nomura AM, Hankin JH, Kolonel IN, Lee J. An epidemiologic study on the association between diet and breast cancer. J Natl Cancer Inst 1987; 78: 595-600.

23. Katsouyanní K, Willett W, Trichopoulos D. Boyle P, Trichopoulou A, Vasilaros S, Papadiamantis J, MacMahon B. Risk of breasu cancer among Greek women in relation to nutrient intake. Cancer 1988; 61: 181-185.

24. Rohan TE, McMichael Al, Baghurst PA. A population-based case-control study of diet and breast cancer in Australia. An J Epidemiol 1988; 128: 478-489.

25. Pryor M, Slattery MI, Robison I,M, Egger M. Adolescent diet and breast cancer in Utah. Cancer Res 1989; 49: 2161-2167.

26. Iscovich JM, Iscovich RB, Howe G, Shiboski S, Kaldor JM. A case-control study of diel and breast cancer in Argentina. Ini J Cancer 1989; 44: 770-776.

27. Graham S, Hellmann R, Marshall J, Freudenheim J, Vena J, Swanson M, Zielezny M, Nemoto T, Stubbe N, Raimondo T. Nutritional epidemiology of postmenopausal breast cancer in Western New York. Am I Epidemiol 1991; 134: $552-566$.

28. Lee HP, Gourley L, Duffy SW, Esteve J, Lee J, Day NE. Dietary effects on breast-cancer risk in Singapore. Lancet 1991; 337: 1197-1200.

29. Zaridze D, Lifanova Y, Maximovitch D, Day NE, Duffy SW. Diet, alcohol consumption and reproductive factors in a case-control study of breast cancer in Moscow. Int J Cancer 1991; 48: 493-501.

30. Gootwin PJ, Boyd NF. Critical appraisal of the evidence that dietary fat intake is related to breast cancer risk in humans. J Natl Cancer Inst 1987; 79:473-485.

31. Lubin JH, Burns PE, Blot WJ, Ziegler RG, Lees AW, Fraumeni JF. Dietary factors and breast cancer risk. Int J Cancer 1981; 28: 685-689.

32. Hislop TG, Coldman N, Elwood JM, Brauer G, Kan L. Childhood and recent eating patterns and risk of breast cancer. Cancer Detect Prev 1986; 9: 47-58.

33. D'Avanzo B, Negri E, Gramenzi A, Franceschi S, Parazini F, Boyle P, La Vechia C. Fats in seasoning and breast cancer risk: an Italian case-control study. Eur J Cancer 1991; 27: 420-423.

34. Talanini R, La Vechia C, Decarli A, Franceschi S, Grattoni E, Grigoletto E, Liberati A, Tognoni G. Social factors, diet and breast cancer in a northern Italian population. Br J Cancer 1984; $49: 723$ 729.

35. IA MG, Moulton L,H, Mill C, Kramer A. Consumption of dairy produce and alcohol in a casecontrol study of bresist cancer. J Natl Cancer Inst 1986; 77: 633-636.

36. Phillips RL. Role of life-style and dietary habits in risk of cancer among Seventh-Day Adventists. Cancer Res 1975: 35: 3513-3522.

37. Zemla B. The role of selected dietary elements in breasi cancer risk among native and migrant populations in Poland. Nutr Cancer 1984; 6: 187-195.

38. Katsouyanni K, Trichopoulos D, Boyle P, Xirouchaki E, Trichopoulou A, Lisseos B, Vasilaros S, MacMahon B. Diet and breast cancer: a case-control study in Greece. Ini J Cancer 1986; $38: 815$ 820.

39. Howe GR, Hirohata T, Hislop TG, Iscovich JM, Yuan JM, Katsouyanni K, Lubin F, Marubini E, Modan B, Rohan T, Toniolo P. Shunzhang, Y. Dictary factors and risk of breasi cancer: combined analysis of 12 case-control studies. J Natl Cancer Inst 1990; 82: 561-569.

40. Hirayama T. Epitemiology of breast cancer with special reference to the role of diet. Prev Med 1978; 7: 173-195.

41. Kinlen LJ. Meat and fal consumption and cancer mortality: a study of strict religious orders in Britain. Lancet 1982; i: 946-949.

42. Phillips RI, Snowdown. DA. Association of meat and coffee use with cancers of the large bowel, breast, and prostate among Seventh-Day Adventists: preliminary results. Cancer Res 1983; 43: $2403 \mathrm{~s}-2408 \mathrm{~s}$. 
43. Willett WC, Stampfer MJ, Colditz GA, Rosner BA, Hennekens CH, Speizer FE. Dietary fat and the risk of breast cancer. N Engl J Med 1987; 316: 22-28.

44. Jones DY, Schatzkin A, Green SB, Block G, Brinton LA, Ziegler RG, Hoover R, Taylor PR. Dietary fat and breast cancer in the National Health and Nutrition Examination Survey I Epidemiologic Follow-up Study. J Natl Cancer Inst 1987; 79: 465-471.

45. Mills PK, Beeson WL, Phillips RL, Fraser GE. Dietary habits and breast cancer incidence among Seventh-day Adventists. Cancer 1989; 64: 582-590.

46. Knekt P, Albanes D, Seppanen R, Aromaa A, Jarvinen R, Hywonen L, Teppo L, Pukkala E. Dietary fat and risk of breast cancer. Am J Clin Nutr 1990; 52: 903-908.

47. Vatten $L J$, Solvoll $K$, Loken EB. Frequency of meat and fish intake and risk of breast cancer in a prospective study of 14,500 Norwegian women. Int J Cancer 1990; 46: 12-15.

48. Howe GR, Friedenreich CM, Jain M, Miller AB. A cohort study of fat intake and risk of breast cancer. J Natl Cancer Inst 1991; 83: 336-340.

49. Van den Brandt PA, Goldbohm RA, Van ' $t$ Veer P, Volovics A, Hermus RJ, Sturmans F. A largescale prospective cohort study on diet and cancer in the Netherlands. J Clin Epidemiol 1990; 43: 285-295.

50. Prentice RL A case-cohort design for epidemiologic studies and disease prevention trials. Biometrika 1986; 73: 1-11.

51. Van den Brandt PA, Schouten LJ, Goldbohm RA, Dorant E, Hunen PMH. Development of a record linkage protocol for use in the Dutch cancer registry for epidemiological research. Ini J Epidemiol 1990; 19: 553-558.

52. Centraal Bureau voor de Statistiek. Sterfte, 1982-1986. Maandstatistick Bevolking 1987; 9: 36-39.

53. Breslow NE, Day NE. Statistical Methods in Cancer Research. II. The Design and Analysis of Cohort Studies. IARC Scientific Publication No. 82, 1987; Lyon: International Agency for Research on Cancer.

54. Bausch-Goldbohm RA, Van den Brandt PA, Dorant E. Hel gebruik van geregistreerde ziekenhuisontslag-diagnoses voor de planning van epidemiologisch onderzoek. (Abstract). Tijdschrift Sociale Gezondheidszorg, 1990; 68: 32.

55. Bausch-Goldbohm RA, Van den Brandt PA, Van 't Veer P, Sturmans F, Hermus RJ. Results of the methodological study for the design of a simplified, self-administered questionnaire. In: E. Riboli, and R. Saracci (eds.), Diet, Hormones and Cancer: Methodological Issues for Prospective Studies. IARC Technical Report No. 4, pp 79-89. Lyon: International Agency for Research on Cancer, 1988.

56. Stichting NEVO. NEVO-tabel; Nederlands Voedingsstoffenbestand 1986-1987. The Hague: Voorlichtingsbureau voor de Voeding, 1986.

57. Willetı W, Stampfer MJ. Total energy intake: implications for epidemiologic analyses. Am J Epidemiol 1986; 124: 17-27.

58. Self SG, Prentice RL. Asymptotic distribution theory and efficiency results for case-cohort studies. Ann Stat 1988; 16: 64-81.

59. Baker RJ. Glim 3.77 Reference Manual. Oxford: Numerical Algorithms Group, 1985.

60. Howe GR, Miller AB, Jain M. Re: "Total energy intake: implications for epidemiologic analyses" (letter). Am J Epidemiol 1986; 124: 157-159.

61. Phillips AN, Pocock SJ. Sample size requirements for prospective studies, with examples for coronary heart disease. J Clin Epidemiol 1989; 42: 639-648.

62. Carroll KK. Lipids and carcinogenesis. J Environ Pathol Toxicol 1980; 3: 253-271.

63. Wynder EL, Chan PC, Cohen LA, MacCormack F, Hill P. Etiology and prevention of breast cancer. In: E. Grundman and L. Beck (eds.), Cancer Campaign, Early Diagnosis of Breast Cancer, Vol 1, pp 1-28. New York: Gustav Fisher Verlag, 1976.

64. Willett W. Nutritional Epidemiology. New York: Oxford University Press, 1990.

65. Klurfeld DM, Weber MM, Kritchevsky D. Inhibition of chemically induced mammary and colon tumor promotion by caloric restriction in rats fed increased dietary fat. Cancer Res. 1987; 47: 2759. 2762.

66. Albanes D. Total calories, body weight, and tumor incidence in mice. Cancer Res 1987; 47: 1987-1992.

67. Pariza M.W. Dietary fat, calorie restriction, ad libitum feeding, and cancer risk. Nutr Rev 1987; 45: $1-7$.

68. Willett WC, Hunter DJ, Stampfer MJ "Colditz GA, Rosner B, Hennekens CH, Manson J, Speizer FE. Dietary fat and breast cancer: an 8-year follow-up. (Abstract). Am J Epidemiol 1991; 134: 715. 
Appendix-table. Percentile values of daily intake of energy and fat (quintile boundaries plus medians within quintiles) in female subcohort members with complete dietary data $(\mathrm{n}=1598)$.

Variable

\section{Percentile value}

\begin{tabular}{|c|c|c|c|c|c|c|c|c|c|}
\hline \multirow{2}{*}{ Vante } & & & & & & & & & \\
\hline & $10 \%$ & $20 \%$ & $30 \%$ & $40 \%$ & $50 \%$ & $60 \%$ & $70 \%$ & $80 \%$ & $90 \%$ \\
\hline Energy (kcal) & 1215 & 1347 & 1455 & 1540 & 1651 & 1751 & 1862 & 2006 & 2220 \\
\hline \multicolumn{10}{|l|}{ Total fat (g) } \\
\hline Unadjusted & 47.0 & 54.5 & 60.4 & 66.6 & 71.9 & 77.9 & 84.0 & 92.4 & 104.8 \\
\hline Energy-adjusted & 61.0 & 66.1 & 69.1 & 71.8 & 74.1 & 76.7 & 78.9 & 82.0 & 86.5 \\
\hline \multicolumn{10}{|l|}{ Saturated fat (g) } \\
\hline Unadjusted & 18.4 & 21.5 & 23.9 & 26.3 & 28.6 & 30.9 & 33.6 & 37.5 & 42.9 \\
\hline Energy-adjusted & 23.0 & 25.1 & 26.6 & 27.9 & 29.2 & 30.5 & 32.2 & 34.1 & 37.4 \\
\hline \multicolumn{10}{|l|}{ Monounsat. fal (g) } \\
\hline Unadjusted & 17.1 & 20.2 & 22.3 & 24.3 & 26.4 & 28.4 & 31.0 & 34.4 & 39.7 \\
\hline Energy-adjusted & 21.8 & 23.7 & 25.0 & 26.2 & 27.4 & 28.6 & 29.8 & 31.2 & 33.8 \\
\hline \multicolumn{10}{|l|}{ Polyunsai. fan (g) } \\
\hline Unadjusted & 6.8 & 8.7 & 10.3 & 11.7 & 13.7 & 15.9 & 18.2 & 21.1 & 26.0 \\
\hline Energy-adjusted & 8.1 & 10.2 & 11.6 & 12.8 & 14.3 & 15.8 & 17.8 & 20.2 & 23.5 \\
\hline \multicolumn{10}{|l|}{ Cholesterol (nig) } \\
\hline Unädjusted & 150 & 177 & 194 & 211 & 228 & 246 & 265 & 290 & 333 \\
\hline Ënergy-adjusted & 163 & 188 & 204 & 219 & 232 & 246 & 261 & 282 & 306 \\
\hline
\end{tabular}




\title{
Chapter 12
}

\section{Prospective study on alcohol consumption and the risk of cancer of the colon and rectum*}

\author{
R. Alexandra Goldbohm ${ }^{1.2}$, Piet A. van den Brandt ${ }^{2}$, \\ Pieter van 't Veer', Elisabeth Dorant ${ }^{2}$, Ferd Sturmans', Rudolph J.J. Hermus'. \\ 1) TNO Toxicology and Nutrition Institute, Zeist, the Netherlands; 2) University of Limbure Department of \\ Epidemiology, Maastricht, the Netherlands
}

\begin{abstract}
The association between alcohol consumption and cancer of the colon and rectum was investigated in a prospective cohort study, conducted in the Netherlands from 1986 onwards among 120,852 men and women, aged 55-69. During 3.3 years of follow-up, 312 and 166 cases of colon and rectal cancer had accumulated respectively. After exclusion of cases diagnosed in the first year of follow-up, the analysis was based on 217 incident cases of colon cancer (107 men and 110 women) and 113 cases of rectal cancer (75 men and 38 women). For colon cancer, no association with intake of alcohol nor with the consumption of beer and wine could be demonstrated; for liquor a significant $(p=0.042)$ decreasing risk with Increasing consumption was observed. For rectal cancer in men, positive trends were observed for alcohol intake $(p=0.041)$, beer $(p=0.050)$ and liquor $(p=0.056)$. Multivariate models including alcohol Intake and one beverage type at a time showed that the Increased risk was mainly restricted to consumption of beer (RR (yes/no): 1.94, 95\% confidence interval: 1.09-3.47). Results for rectal cancer in women were consistent with those in men, but data were too scarce to provide stable estimates. It is concluded that only consumption of beer appeared to increase risk of rectal cancer, but not colon cancer. It is speculated whether the high nitrosamine content of beer in the past has caused the Increased risk.
\end{abstract}




\section{Introduction}

The consumption of large amounts of alcohol has definitely been shown to increase the risk for cancer of the upper digestive tract (oral cavity, pharynx, esophagus). For cancer of the lower intestinal tract, i.e. colon and rectum, substantial evidence has accumulated from epidemiological studies that alcohol consumption has only a weak effect on risk, if any. A recently published meta-analysis, performed on presumably all 27 epidemiologic studies regarding colorectal cancer that quantified alcohol consumption, demonstrated a relative risk (RR) of only 1.10 (95\% confidence interval (CI) 1.04-1.14) for subjects taking two drinks per day (equivalent to $24 \mathrm{~g}$ of ethanol) compared to abstainers; the association in follow-up studies, however, was stronger ( $R R=1.32, C I 1.16-1.51)$ than that in case-control studies ( $R R=1.07, C I 1.02-1.12)(1)$. In the meta-analysis, no difference in RR between men and women was observed, nor between tumor sites (colon or rectum). When type of alcoholic beverage was evaluated (in 8 of the 27 studies), consumption of beer had the largest relative risk $(1.26, \mathrm{CI}$ 1.13-1.41) as compared to wine $(\mathrm{RR}=1.11$, $\mathrm{CI} 0.91-1.36)$ or liquor $(\mathrm{RR}=1.13, \mathrm{CI}$ 0.99-1.29). Nevertheless, a number of studies have convincingly shown a higher relative risk for rectal cancer than for colon cancer (2-7). It is furthermore intriguing that in some studies beer drinking is clearly associated with an increased relative risk of rectal cancer, $(2,3,5,6,8,9)$ while in other studies it is not $(4,10,11)$. It has been suggested that the higher risk associated with beer drinking has been caused by contamination of many types of beer with nitrosamines in the past (12).

These results prompted us to investigate the relation between consumption of alcoholic beverages and the risk of colon and rectal cancer in the Dutch prospective cohort study on diet and cancer (13). In this study among 120,000 men and women, which started in 1986, detailed information has been collected on dietary habits including drinking habits by means of a self-administered questionnaire.

\section{Subjects and methods}

\section{The cohort}

The prospective cohort study on diet and cancer has been initiated in the Netherlands in September 1986. The cohort included 58,279 men and 62,573 women aged 55-69 at the start of the study. The study population originated from 204 municipal population registries throughout the country. At baseline, the cohort members completed a mailed, self-administered questionnaire on dietary habits and other risk factors for cancer. For data processing and analysis the case-cohort approach was used: the cases were enumerated for the entire cohort, while the person years at risk accumulated in the cohort were estimated from a random sample (subcohort). This subcohort of 3500 subjects (1688 men, 1812 women) was sampled from the cohort after the baseline measurement and was followed up for vital status over 3.3 years. The study design has been described in detail elsewhere (13).

\section{Follow-up for cancer}

Follow-up for incident cancer was established by computerized record linkage with all nine regional cancer registries in the Netherlands and with PALGA, the Dutch national data base of pathology reports. The method of record linkage has been published previously (14). The present analysis is restricted to cancer incidence in the period from September 1986 (baseline measurement) to December 1989, i.e. a followup period of 3.3 years. In this period, completeness of follow-up of the cohort through linkage with the cancer registries and PALGA together was estimated to be very high 
(15). After excluding subjects who reported a history of cancer other than skin cancer in the baseline questionnaire, a total of 478 incident cases with microscopically confirmed primary adenocarcinoma of the colon (157 men, 155 women) and rectum (101 men, 65 women) were identified.

\section{Questionnaire}

A self-administered questionnaire was used at baseline to collect data on dietary habits, precursors and other (possible) risk factors for colon, rectal and other cancers. The dietary section of the questionnaire concentrated on habitual consumption of food and beverages during the year preceding the start of the study and included 150 food items. Use of alcoholic beverages was addressed by six items: (a) beer, (b) red wine, (c) white wine, (d) sherry and other fortified wines, (e) liqueur (containing $16 \%$ ethanol) and (f) (Dutch) gin, brandy, whiskey. Together, these items presumably covered all types of alcoholic beverages consumed. For each item, the questionnaire included seven frequency categories (never/less than once per month, once per month, 2-3 times per month, once per week, 2-3 times per week, 4-5 times per week, 6-7 times per week) and the number of glasses per consumption frequency.

The questionnaire has been validated against a 9-day diet record (16). The Spearman correlation coefficient between mean daily ethanol intake assessed by the questionnaire and that estimated from the 9-day record was 0.89 for all subjects and 0.85 for users of alcoholic beverages; the absolute amount of ethanol reported in the questionnaire by users of alcoholic beverages was, on average, $86 \%$ of that reported in the record.

\section{Data analysis}

Questionnaire data were processed for all 478 incident colorectall cases in the cohort and for all subcohort members. After excluding prevalent cancer cases other than skin cancer from the subcohort, 3346 subjects (1630 men and 1716 women) remained in this group. The questionnaire data were key-entered twice and processed in a manner blinded with respect to case/subcohort status in order to minimize observer bias in coding and interpretation of the data. Alcohol data were considered incomplete when all questions on consumption frequency of alcoholic beverages were left blank and two other questions on alcohol consumption did not indicate that the subject was an abstainer. These two questions concerned alcohol intake during the past week and five years ago. The analysis is based on 3170 subcohort members (94.7\%) and $46 \mathrm{i}$ cases $(96.4 \%)$ for whom the data on alcohol consumption were considered complete. An additional $4.3 \%$ of the subjects produced incomplete or inconsistent dietary data, according to criteria described by Goldbohm et al (16). For these subjects, a dummy variable was included in the models adjusting for dietary factors.

For each alcoholic beverage item, the number of glasses taken per week was calculated by multiplying the frequency per week by the number of glasses per frequency. Four items from the questionnaire, i.e. red wine, white wine, sherry and liqueur, were combined in this and subsequent analyses, since these items were substantially correlated and separate treatment would eventually result in scarcity of data. Mean daily ethanol and nutrient intakes were calculated using the computerized Dutch food composition table (17). Energy adjustment of nutrient. intakes was done according to Willett and Stampfer (18). The Quetelet Index $\left(\mathrm{kg} / \mathrm{m}^{2}\right)$ was used as a measure of obesity.

Data were analysed using the case-cohort approach, (19), assuming exponentially distributed survival times in the follow-up period. Since standard software was not available for this type of analysis, specific programs were developed to account for the 
additional variance introduced by sampling from the cohort instead of using the entire cohort (20). The following variables were considered as potential confounders: age, large-bowel cancer in first-degree relatives, smoking. Quetelet index, levell of education, previous gallbladder surgery, intake of energy and energy-adjusted intake of fat, meat protein, dietary fiber, vitamin C and calcium. Subclinical symptoms of large bowel cancer may influence dietary habits before diagnosis. Therefore, we excluded cases diagnosed in the first year of follow-up after assessing the impact of exclusion on the risk estimates.

\section{Results}

Table 1. Drinking habits among subcohort and cases of colon and rectal cancer.

Men! Women

Drinking habit

$\begin{array}{lllllll}\text { Subcohort } & \text { Colon } & \text { Rectum } & \text { Subcohort, } & \text { Colon } & \text { Rectum } \\ \text { n. PT } & \text { n } & \text { n } & \text { n } & \text { PT† } & \text { n } & \text { n }\end{array}$

\section{Total*}

Abstainers.

Users of alcoholic beverages:

Ethanol (g/day)
$0.1-4.9$
$5.0-14.9$
15.0-29.9
$\geq 30.0$

Beer (glasses/week)

No beer

$<1.1$

i. $1-4.9$

$\geq 5.0$

Wine (glasses/week) $\ddagger$

$$
\begin{gathered}
\text { No wine } \\
<1.2 \\
1.2-4.9 \\
\geq 5.0
\end{gathered}
$$

I_iquor (glasses/week)

$$
\begin{gathered}
\text { No liquor } \\
\leq 2.0 \\
2.1-7.4 \\
\geq 7.5
\end{gathered}
$$

$\begin{array}{rrrrrrrr}1591 & 5114 & 153 & 96 & 1579 & 5144 & 146 & 57 \\ 246 & 782 & 27 & 14 & 513 & 1667 & 56 & 19\end{array}$

$\begin{array}{rrrr}325 & 1043 & 30 & 24 \\ 441 & 1418 & 30 & 17 \\ 358 & 1167 & 39 & 23 \\ 221 & 704 & 27 & 18\end{array}$

$\begin{array}{rrrr}582 & 1907 & 42 & 21 \\ 286 & 933 & 25 & 9 \\ 142 & 458 & 16 & 5 \\ 56 & 179 & 7 & 3\end{array}$

$\begin{array}{rrrr}435 & 1396 & 45 & 24 \\ 344 & 1107 & 26 & 23 \\ 229 & 746 & 19 & 15 \\ 337 & 1083 & 36 & 20\end{array}$

$\begin{array}{rrrr}921 & 3005 & 75 & 30 \\ 91 & 297 & 7 & 6 \\ 25 & 79 & 4 & 1 \\ 28 & 92 & 4 & 1\end{array}$

$\begin{array}{rrr}554 & 1777 & 42 \\ 251 & 806 & 22 \\ 271 & 879 & 24 \\ 268 & 867 & 38\end{array}$

38
15
15
14

$\begin{array}{rrrr}58 & 188 & 9 & 3 \\ 353 & 1158 & 24 & 11 \\ 323 & 1056 & 22 & 10 \\ 327 & 1059 & 35 & 14\end{array}$

$\begin{array}{rrrr}435 & 1402 & 38 & 24 \\ 311 & 1005 & 32 & 17 \\ 285 & 924 & 26 & 16 \\ 313 & 998 & 29 & 25\end{array}$

$\begin{array}{rrrr}855 & 2789 & 77 & 35 \\ 102 & 335 & 6 & 1 \\ 65 & 209 & 2 & 1 \\ 42 & 137 & 5 & 1\end{array}$

- Total number of subjects for whom information on drinking habits was available.

+ PT: Person-time-at-risk (year) in the subcohort; multiplying PT by the inverse of the subcohort sampling fraction $(1 / 0.029=34.533)$ gives the estimated person-time-at-risk in the cohort, which may be used to calculate incidence density rates.

† Among male wine drinkers, $63 \%$ drank red wine, $59 \%$ white wine, $48 \%$ sherry and $16 \%$ liqueur; among female wine drinkers, the corresponding percentages were $47,54,51$ and 38 respectively. 
Table 1 displays the drinking habits in the study population. Of the men in the subcohort, $85 \%$ drank alcoholic beverages at least once a month. Consumption of beer, wine and liquor was about equally distributed. Liquor was consumed in larger amounts than beer or wine. In contrast, $68 \%$ of the women reported drinking of alcoholic beverages, mainly wine. Their intake of alcohol (mean 8.5, SD $10.6 \mathrm{~g}$ /day) was also much lower than that of male alcohol consumers (mean 17.1, SD $16.6 \mathrm{~g} /$ day).

Table 2 shows that the three types of alcoholic beverage were uncorrelated. The correlation of the beverage type with ethanol intake indicates their relative importance in men (liquor) and women (wine). Since alcohol provides energy, alcohol and energy intake are modestly correlated $(r=0.14)$. The very low correlation with energy derived from other sources indicates that alcohol is merely added to the diet without affecting energy intake from other sources.

Table 2. Associations between ethanol intake, consumption of specific alcoholic beverages and energy in the subcohort (Pearson (Rp) and Spearman (Rs) correlation coefficients).

\begin{tabular}{|c|c|c|c|c|c|c|c|c|c|}
\hline & \multicolumn{2}{|c|}{ Ethanol } & \multicolumn{3}{|l|}{ Beer } & \multicolumn{2}{|l|}{ Wine } & \multicolumn{2}{|l|}{ Liquor } \\
\hline & $\mathrm{Rp}$ & Rs & $\mathrm{Rp}$ & R & & Rp & Rs & $\mathrm{Rp}$ & Rs \\
\hline \multicolumn{10}{|l|}{ Men $(n=1591)$} \\
\hline Ethanol & 1.00 & & & & & & & & \\
\hline Beer & 0.51 & $(0.52)$ & 1.00 & & & & & & \\
\hline Wine & 0.50 & $(0.41)$ & 0.01 & ( & $0.08)$ & 1.00 & & & \\
\hline Liquor & 0.73 & $(0.69)$ & 0.02 & ( & $0.16)$ & 0.04 & $(0.07)$ & 1.00 & \\
\hline Energy & 0.15 & $(0.14)$ & 0.13 & ( & 0.17 ) & 0.07 & $(0.06)$ & 0.07 & $(0.09)$ \\
\hline Energy excl. ethanol & -0.07 & $(-0.05)$ & 0.02 & ( & 0.08 ) & -0.04 & $(-0.01)$ & -0.09 & $(-0.05)$ \\
\hline \multicolumn{10}{|l|}{ Women $(\mathrm{n}=1579)$} \\
\hline Ethanol & 1.00 & & & & & & & & \\
\hline Beer & 0.24 & $(0.33)$ & 1.00 & & & & & & \\
\hline Wine & 0.84 & $(0.90)$ & 0.05 & ( & 0.20 ) & 1.00 & & & \\
\hline Liquor & 0.57 & $(0.43)$ & 0.04 & ( & $0.16)$ & 0.07 & $(0.16)$ & 1.00 & \\
\hline Energy & 0.14 & $(0.14)$ & 0.03 & ( & 0.09 ) & 0.14 & $(0.14)$ & 0.05 & $(0.10)$ \\
\hline Energy excl. ethanol & -0.02 & $(0.02)$ & -0.01 & ( & 0.03 ) & 0.07 & $(0.04)$ & -0.04 & $(0.02)$ \\
\hline
\end{tabular}

Table 3 presents mean alcohol intake in the subcohort according to several characteristics that are considered to be confounding variables for the relationship between a.jcohol intake and colorectal cancer. Smoking and education were strongly, positively related to alcohol intake. The Quetelet index was positively related to alcohol intake in men only; in women a weak inverse association was found. Alcoholl intake was relatively constant across quintiles of absolute fat intake, but decreased with increasing level of energy-adjusted fat intake (data not shown), because alcohol contributes to energy intake. Dietary fiber was negatively associated with alcohol intake. The intake of protein from meat increased with alcohol consumption, which relation persisted after adjustment for energy intake.

Table 4 shows the relative rates (RR) for all cases of cancer of the colon and rectum according to alcohol consumption and for those cases diagnosed after one year of follow-up. 
Table 3. Mean ethanol intake ( $\mathrm{g} / \mathrm{day}$ ), adjusted for age, according to baseline characteristics of men and. women in the subcohort".

Characteristic

Men

Women

Mean SD

55-59

60-64

65-69

Smoking

Never

Fx-smokert

Current cigarette smoker

$<10 /$ day

10-19/day

$\geq 20 /$ day

Quetelet index $\left(\mathrm{kg} / \mathrm{m}^{2}\right)$

$\leq 22$
$23-24$
$25-26$
$\geq 27$

Gallbladder surgery

No

Yes

L.evel of education

Low

Medium

High

Fat intake

Ouintile 1

Ouintile 2

Quintile 3

Ouintile 4

Ouintile 5

Meat protein intake

Ouintile 1

Duintile 2

Quintile 3

Duintile 4

Quintile 5

Dietary fiber intake

Quintile 1

Quintile 2

Quintile 3

Ouintile 4

Quintile 5
575

526

407

$14.8 \dagger$
$15.2 \dagger$
$13.4 t$

146

825

7.9

15.1

$83 \quad 16.2$

265

189

16.7

21.6

298

475

394

287

13.1

16.9

15.1

16.8

1440

68

15.7

13.5

702.

525

271

13. 3

16.3

20.2

303

300

302

300

303

16.4

14.0

16.1

16.7

1.4 .6

299.

302 :

302

302

3013

11.8
13.8
14.9
18.4
18.9

305 :

296

309

301.

297

18.1
16.3
16.2
14.0
13.1

16.9

17.5

14.9

589

515

415

$6.3+$

$5.6 \dagger$

$5.2+$

10.3

9.3

8.8

6.6

14.9

890

3.6

8.7

10.9

15.6

18.7

21.5

103

8.9

11.5

134

7.6

14.1

10.6

16.0

15.3

398.

6.9

9.8

18.4

420.

289

6.5

9.8

15.2

368

5.2

9.0

9.7

$16.7 \quad 1316$

12.9203

6.1

9.7

5.2

8.4

4.1

839

18.6

17.6

537

135

4.4

7.7

7.5

10.9

$9.8 \quad 12.2$

$5.5 \quad 10.5$

$6.0 \quad 9.0$

$5.2 \quad 8.4$

$5.6 \quad 9.2$

$7.4 \quad 10.4$

$18.4 \quad 303$

$15.8 \quad 308$

10.4

4.1

14. 1

16.1

16.0

20.7

307

306

298

304

304

3.9

7.5

$15-7.2$

$6.7 \quad 10.2$

$\begin{array}{ll}7.3 & 10.8\end{array}$

$7.4 \quad 10.9$

$\begin{array}{ll}18.5 & 297 \\ 16.4 & 308 \\ 18.6 & 308 \\ 14.4 & 305 \\ 13.9 & 301\end{array}$

6.8

11.0

7.1

10.7

$6.1 \quad 8.7$

$\begin{array}{ll}4.8 & 8.1\end{array}$

$\begin{array}{ll}4.8 & 8.8\end{array}$

- Subjects with complete dietary data (1508 men and 1519 women); numbers may not add up to these figures due to missing values for non-dietary variables.

$\dagger$ Ethanol intake not adjusted for age.

₹ Ex-smokers include current smokers of pipe and cigars. 
Table 4. Age-adjusted relative rate of colon and rectal cancer classified according to ethanol intake among all cases and after exclusion of cases diagnosed in the first year of follow-up.

\begin{tabular}{|c|c|c|c|c|c|c|}
\hline \multirow{2}{*}{$\begin{array}{l}\text { Level of ethanol } \\
\text { intake ( } g / \text { day) }\end{array}$} & \multicolumn{3}{|c|}{ All cases } & \multicolumn{3}{|c|}{ First year excluded } \\
\hline & $\mathbf{n}$ & RR & $95 \% \mathrm{CI}$ & n & RR & $95 \% \mathrm{Cl}$ \\
\hline & \multicolumn{6}{|c|}{ COLON } \\
\hline Men total & 153 & & & 107 & & \\
\hline Abstainers & 27 & 1.00 & & 23 & 1.00 & \\
\hline $0.1-4.9$ & 30 & 0.91 & $0.52-1.57$ & 21 & 0.76 & $0.41-1.42$ \\
\hline $5.0-14.9$ & 30 & 0.67 & $0.39-1.15$ & 20 & 0.53 & $0.29-1.00$ \\
\hline $15.0-29.9$ & 39 & 1.02 & $0.61-2.12$ & 26 & 0.81 & $0.45-1.45$ \\
\hline$\geq 30.0$ & 27 & 1.20 & $0.68-2.12$ & 17 & 0.91 & $0.47-1.76$ \\
\hline p-value for trend & \multicolumn{2}{|c|}{0.413} & & \multicolumn{2}{|c|}{0.771} & \\
\hline Women total & 146 & & & 110 & & \\
\hline Abstainers & 56 & 1.00 & & 45 & 1.00 & \\
\hline $0.1 \cdot 4.9$ & 42 & 0.67 & $0.45-1.00$ & 31 & 0.62 & $0.39-0.97$ \\
\hline $5.0-14.9$ & 25 & 0.81 & $0.50-1.29$ & 16 & 0.64 & $0.36-1.14$ \\
\hline $15.0-29.9$ & 16 & 1.07 & $0.61-1.86$ & 13 & 1.09 & $0.59-2.03$ \\
\hline$\geq 30.0$ & 7 & 1.31 & $0.60-2.87$ & 5 & 1.22 & $0.48-3.09$ \\
\hline \multirow[t]{2}{*}{$\mathrm{p}$-value for trend } & \multicolumn{2}{|c|}{0.754} & & \multicolumn{2}{|c|}{0.938} & \\
\hline & \multicolumn{6}{|c|}{ RECIUM } \\
\hline Men total & 105 & & & 75 & & \\
\hline Abstainers & 14 & 1.00 & & 8 & 1.00 & \\
\hline $0.1-4.9$ & 24 & 1.36 & $0.69-2.67$ & 16 & 1.58 & $0.70-3 ; 61$ \\
\hline $5.0-14.9$ & 17 & 0.71 & $0.35 \cdot 1.46$ & 12 & 0.87 & $0.37-2.80$ \\
\hline $15.0-29.9$ & 23 & 1.14 & $0.58-2.26$ & 22 & 1.90 & $0.86-4.19$ \\
\hline$\geq 30.0$ & 27 & 1.51 & $0.74 \cdot 3.09$ & 17 & 2.49 & $1.10-5.66$ \\
\hline p-value for trend & \multicolumn{2}{|c|}{0.479} & & \multicolumn{2}{|c|}{0.021} & \\
\hline Women total & \multicolumn{2}{|l|}{57} & & \multicolumn{2}{|l|}{38} & \\
\hline Abstainers & 19 & 1.00 & & 13 & 1.00 & \\
\hline $0.1-4.9$ & 21 & 0.99 & $0.54-1.82$ & 13 & 0.90 & $0.42-1.93$ \\
\hline $5.0-14.9$ & 9 & 0.86 & $0.39 \cdot 1.87$ & 6 & 0.84 & $0.32-2.20$ \\
\hline $15.0-29.9$ & 5 & 0.99 & $0.38-2.61$ & 4 & 1.17 & $0.38-3.59$ \\
\hline$\geq 30.0$ & 3 & 1.71 & $0.52-5.68$ & 2 & 1.74 & $0.39-7.76$ \\
\hline $\mathrm{p}$-value for trend & \multicolumn{2}{|c|}{0.781} & & \multicolumn{2}{|c|}{0.685} & \\
\hline
\end{tabular}

Among men, the estimated relative rates in cases diagnosed after more than one year of follow-up were clearly different from those in all cases, although among colon cancer cases relatively more heavy drinkers and less abstainers were diagnosed in the first year of follow-up, whereas among rectal cancer cases an opposite trend was detected. Further exclusion of cases diagnosed in the second year of follow-up did not change the estimates anymore. Among women, no effect of exclusion of cases diagnosed in the first year was seen. For subsequent analyses, we excluded male as well as female cases diagnosed in the first year of follow-up.

Tables 5 and 6 present the relative rates of colon and rectal cancer for ethanol intake and the three types of alcoholic beverage after adjustment for confounding. Familial history of large-bowel cancer, vitamin $\mathrm{C}$ and calcium were not included in the models since they did not affect the estimates for alcohol. The estimated RR's were 
quite similar for men and women, justifying pooling of the data of both sexes. For colon cancer no enhancing effect of alcohol of any type was evident. Notable are the Ushaped dose-effect relations for ethanol intake and all beverage types except liquor, which showed a significant $(\mathrm{p}=0.042)$ negative association. The risk of rectal cancer, on the contrary, appeared to be enhanced by alcohol consumption and, most consistently, by beer consumption.

Table 5. Relative rate of colon cancer* according to drinking habits, adjusted for confounderst.

Drinking habits

Men Women Both sexes

$\begin{array}{llllll}\text { n } & \text { RR } & \text { n } & \text { R } & \text { RR } & 95 \% \text { CI }\end{array}$

\begin{tabular}{|c|c|c|c|c|c|c|c|}
\hline Abstainers! & 22 & 1.00 & 41 & 1.00 & 63 & 1.00 & \\
\hline \multicolumn{8}{|l|}{ Ethanol(g/day) } \\
\hline $0.1-4.9$ & 20 & 0.72 & 31 & 0.69 & 51 & 0.70 & $0.47-1.06$ \\
\hline $5.0-14.9$ & 19 & 0.52 & 15 & 0.61 & 34 & $0.58^{\circ}$ & $0.37-0.93$ \\
\hline $15.0-29.9$ & 24 & 0.79 & 12 & 1.01 & 36 & 0.89 & $0.49-1.60$ \\
\hline$\geq 30.0$ & 16 & 0.94 & 5 & 1.29 & 21 & 1.09 & $0.33-3.59$ \\
\hline p-value for trend & \multicolumn{2}{|c|}{0.767} & \multicolumn{2}{|c|}{0.756} & \multicolumn{2}{|c|}{0.787} & \\
\hline \multicolumn{8}{|l|}{ Beer (glasses/week) } \\
\hline No beer & 33 & 0.84 & 51 & 0.68 & 84 & 0.74 & $0.52-1.05$ \\
\hline$<1.1$ & 12 & 0.39 & & & 17 & 0.46 & $0.25-0.83$ \\
\hline $1.1-4.9$ & 12 & 0.66 & 121 & $1.07 \rrbracket$ & 16 & 0.83 & $0.44-1.56$ \\
\hline$\geq 5.0$ & 22 & 0.88 & & & 25 & 0.94 & $0.41-2.14$ \\
\hline p-value for trend & \multicolumn{2}{|c|}{0.450} & \multicolumn{2}{|c|}{0.464} & \multicolumn{2}{|c|}{0.592} & \\
\hline \multicolumn{8}{|c|}{ Wine (glasses/week) } \\
\hline No wine & 27 & 0.60 & 6 & 1.41 & 33 & 0.70 & $0.41-1.20$ \\
\hline$<1.2$ & 14 & 0.65 & 18 & 0.64 & 32. & 0.65 & $0.42-1.02$ \\
\hline $1.2-4.9$ & 15 & 0.63 & 16 & 0.63 & 31 & 0.63 & $0.40-0.99$ \\
\hline$\geq 5.0$ & 23 & 1.05 & 23 & 0.81 & 46 & 0.96 & $0.57-1.59$ \\
\hline p-value for trend & \multicolumn{2}{|c|}{0.746} & \multicolumn{2}{|c|}{0.160} & \multicolumn{2}{|c|}{0.464} & \\
\hline \multicolumn{8}{|c|}{ Liquor (glasses/week) } \\
\hline No liquor & 26 & 0.76 & 53 & 0.75 & 79 & 0.76 & $0.54-1.08$ \\
\hline$\leq 2.0$ & 19 & 0.69 & & & 25 & 0.73 & $0.43-1.22$ \\
\hline $2.1-7.4$ & 18 & 0.72 & 101 & 0.581 & 19 & 0.61 & $0.35-1.08$ \\
\hline$\geq 7.5$ & 16 & 0.56 & & & 19 & 0.62 & $0.30-1.28$ \\
\hline p-value for trend & \multicolumn{2}{|c|}{0.114} & \multicolumn{2}{|c|}{0.090} & \multicolumn{2}{|c|}{0.042} & \\
\hline
\end{tabular}

- Cases diagnosed in first year of follow-up excluded; number of cases may not add up to those in Table 4 due to missing values for non-dietary variables.

$\uparrow$ Adjusted for age, smoking, Quetelet index, history of gallbladder surgery, level of education, intake of energy and energy-adjusted intake of fat, meat protein and dietary fiber.

\$. Reference category.

$\$$ Also adjusted for sex.

All three quantitative levels combined. 
Table 6. Relative rate of rectal cancer* according to drinking habits, adjusted for confounderst.

\begin{tabular}{|c|c|c|c|c|c|c|c|}
\hline \multirow[t]{2}{*}{ Drinking habits } & \multicolumn{2}{|c|}{ Men } & \multicolumn{2}{|c|}{ Women } & \multicolumn{3}{|c|}{ Both sexes } \\
\hline & n & RR & n & RR & $\mathbf{n}$ & RR\$ & $95 \% \mathrm{Cl}$ \\
\hline Abstainersł & 7 & 1.00 & 12 & 1.00 & 19 & 1.00 & \\
\hline \multicolumn{8}{|l|}{ Ethanol(g/day) } \\
\hline $0.1-4.9$ & 16 & 1.91 & 10 & 0.83 & 26 & 1.22 & $0.63-2.35$ \\
\hline $5.0-14.9$ & 12 & 1.08 & 5 & 0.71 & 17 & 0.84 & $0.43-1.64$ \\
\hline $15.0-29.9$ & 21 & 2.14 & 4 & 0.97 & 25 & 1.51 & $0.71-3.21$ \\
\hline$\geq 30.0$ & 17 & 2.83 & 2. & 1.31 & 19 & 1.95 & $0.40-9.64$ \\
\hline $\mathrm{p}$-value for trend & \multicolumn{2}{|c|}{0.041} & \multicolumn{2}{|c|}{0.965} & \multicolumn{2}{|c|}{0.091} & \\
\hline \multicolumn{8}{|l|}{ Beer (glasses/week) } \\
\hline No beer & 15 & 1.26 & 15 & $0.7 !$ & 30 & 0.91 & $0.50-1.65$ \\
\hline$\leq 1.1$ & 19 & 2.03 & & & 23. & 1.81 & $0.91-3.58$ \\
\hline $1.1 \cdot 4.9$ & 13 & 2.17 & 61 & 1.911 & 14 & 1.88 & 0.84 .4 .23 \\
\hline$\geq 5.0$ & 19 & 2.04 & & & 20. & 1.66 & $0.57-4.80$ \\
\hline $\mathrm{p}$-value for trend & \multicolumn{2}{|c|}{0.050} & \multicolumn{2}{|c|}{0.594} & \multicolumn{2}{|c|}{0.044} & \\
\hline \multicolumn{8}{|l|}{ Wine (glasses/week) } \\
\hline No wine & 30 & 2.10 & 2. & 1.56 & 32 & 1.59 & $0.83-3.04$ \\
\hline$<1.2$ & 13 & 1.93 & 5 & 0.63 & $18:$ & 1.18 & $0.62-2.24$ \\
\hline $1.2-4.9$ & 9 & 1.13 & 4 & 0.56 & 13 & 0.79 & $0.38-1.63$ \\
\hline$\geq 5.0$ & 14 & 1.70 & 10 & 1.29 & 24 & 1.38 & $0.66-2.89$ \\
\hline p-value for trend & \multicolumn{2}{|c|}{0.972} & \multicolumn{2}{|c|}{0.964} & \multicolumn{2}{|c|}{1.000} & \\
\hline Liquor (glasses/week) & & & & I & & & ร \\
\hline No liquor & 19 & 1.65 & & & & & $0.68-4.00$ \\
\hline$\leq 2.0$ & 13 & 1.60 & & & & & $0.61-4.19$ \\
\hline $2.1-7.4$ & 11 & 1.35 & & & & & $0.50-3.60$ \\
\hline$\geq 7.5$ & 23 & 2.67 & & & & & $0.95-7.52$ \\
\hline $\mathrm{p}$-value for trend & & & & & & & \\
\hline
\end{tabular}

- Cases diagnosed in first year of follow-up excluded; number of cases may not add up to those in Table 4 due to missing values for non-dietary variables.

† Adjusted for age, smoking, Quetelet index, history of gallbladder surgery, level of education, intake of energy and energy-adjusted intake of fat, meat protein and dietary fiber.

†. Reference category.

$\$$ Also adjusted for sex.

I. All three quantitative levels combined.

I Insufficient data for liquor consumption in women; confidence intervals based on estimates for men only.

From the analysis presented in Table 7, which included ethanol intake as well as one type of beverage in the model, it was evident that beer was the only beverage for which risk was significantly increased $(\mathrm{RR}=1.94$, CI 1.09-3.47).

The estimated RR's of colon and rectal cancer for red, white and fortified wines were comparable to those for all wine. The results with respect to alcohol consumption were similar for subjects with and without a history of large-bowel cancer in first-degree relatives. 
Table 7. Relative rate estimates for colon and rectal cancer (men and women combined) in multivariate models including ethanol intake and type of alcoholic beverage*.

\begin{tabular}{|c|c|c|c|c|c|c|}
\hline \multirow[t]{2}{*}{ Variable } & \multicolumn{2}{|c|}{ Beer } & \multicolumn{2}{|c|}{ Wine } & \multicolumn{2}{|c|}{ Liquor } \\
\hline & RR & $95 \% \mathrm{CI}$ & RR & $95 \% \mathrm{CI}$ & RR & $95 \% \mathrm{Cl}$ \\
\hline
\end{tabular}

\section{COLON}

\begin{tabular}{|c|c|c|c|c|c|c|}
\hline \multicolumn{7}{|l|}{ Ethanol(g/day) } \\
\hline 0 & \multicolumn{2}{|l|}{1.00} & \multicolumn{2}{|l|}{1.00} & \multicolumn{2}{|l|}{1.00} \\
\hline $0.1 \cdot 4.9$ & 0.72 & $0.48-1.09$ & 0.67 & $0.39-1.16$ & 0.74 & $0.49-1.12$ \\
\hline $5.0-14.9$ & 0.61 & $0.38-1.00$ & 0.56 & $0.31-1.02$ & 0.65 & $0.40-1.08$ \\
\hline $15.0-29.9$ & 0.95 & $0.50-1.81$ & 0.85 & $0.42-1.75$ & 1.04 & $0.52-2.09$ \\
\hline$\geq 30.0$ & 1.17 & $0.33-4.13$ & 1.05 & $0.28-3.89$ & 1.31 & $0.35-4.94$ \\
\hline p-value for trend & \multicolumn{2}{|c|}{0.893} & \multicolumn{2}{|c|}{0.677} & \multicolumn{2}{|c|}{0.620} \\
\hline Type (yes/no) & 0.86 & $0.57-1.31$ & 1.06 & $0.67-1.67$ & 0.76 & $0.49-1.18$ \\
\hline p-value & \multicolumn{2}{|c|}{0.466} & \multicolumn{2}{|c|}{0.796} & \multicolumn{2}{|c|}{0.173} \\
\hline
\end{tabular}

RECTUM

Ethanol(g/day)

\begin{tabular}{|c|c|c|c|c|c|c|}
\hline 0 & 1.00 & & 1.00 & & 1.00 & \\
\hline $0.1-4.9$ & 1.01 & $0.51-1.98$ & 1.60 & $0.77-3.33$ & 1.26 & $0.66-2.42$ \\
\hline $5.0-14.9$ & $0.6 \mathrm{i}$ & $0.29-1.30$ & 1.10 & $0.51-2.41$ & 0.90 & $0.44-1.87$ \\
\hline $15.0-29.9$ & 1.04 & $0.43-2.53$ & 1.96 & $0.82-4.69$ & 1.66 & $0.66-4.15$ \\
\hline$\geq 30.0$ & 1.31 & $0.23-3.47$ & 2.62 & $0.49-13.9$ & 2.16 & $0.36-12.8$ \\
\hline $\mathrm{p}$-value for trend & \multicolumn{2}{|c|}{0.525} & \multicolumn{2}{|c|}{0.031} & \multicolumn{2}{|c|}{0.066} \\
\hline pe (yes/no) & 1.94 & $1.09-3.47$ & 0.69 & $0.41-1.16$ & 0.87 & $0.50-1.50$ \\
\hline$p$-value & \multicolumn{2}{|c|}{0.012} & \multicolumn{2}{|c|}{0.149} & & \\
\hline
\end{tabular}

- The models included all covariates that were also included in the models in Tables 5 and 6 , the categorical variable ethanol intake and one type of alcoholic beverage (yes/no).

\section{Discussion}

We have presented evidence that supports a positive relationship between alcohol consumption and rectal cancer but not colon cancer. For rectal cancer, the positive association was most pronounced among men and referred to alcohol intake and beer consumption, both showing a significant trend. Liquor consumption showed a significantly increased risk in the highest consumption category, i.e. one or more drinks per day. Multivariate analysis, however, revealed that beer was the only beverage type that accounted for the increased risk. The resuits for women were compatible with those for men, although the data were too scarce to draw definite conclusions with respect to women specifically. For colon cancer, the U-shaped relation with amount of alcohol consumed was very clear in men as well as; women. The lowest relative rate $(\mathbf{R}=0.58)$ was observed for consumption of $5-15 \mathrm{~g}$ of ethanol per day. For types of alcoholic beverage the $\mathrm{U}$-shape seemed to hold as well, except for liquor which showed a significantly negative trend $(p=0.042)$.

Misclassification of alcohol consumers as abstainers can easily occur in selfadministered food frequency questionnaires since some subjects not only tend to skip 
items they do not consume $(16,21)$, but possibly also items found difficult to answer. We have minimized such misclassification by coding subjects who skipped the alcohol items as abstainer only when this was consistent with other information on alcohol consumption. Although the consequence of this choice may have been that the subjects with missing alcohol data $(2.4 \%$ of the men and $8.0 \%$ of the women) comprise a relatively high proportion of actual abstainers, resulting in an underestimation of the proportion of abstainers among the subjects included in the analysis, this is not likely to affect the risk estimate among abstainers.

We did not try to assess lifetime alcohol consumption, which may be a more relevant measure of exposure in the etiology of cancer than alcohol consumption during the past year. Annually repeated administration of the questionnaire over five years, however, has demonstrated that the correlations between the baseline and repeated measurements of alcohol intake remained virtually stable with increasing interval between the measurements (22). Moreover, it is well known from methodological studies on nutrient intake that retrospective assessment of diet in the distant past is strongly influenced by current diet (23-25).

We decided, mainly on biological grounds, to exclude from the analysis all cases diagnosed in the first year after completion of the baseline questionnaire. Exclusion appeared to have effect on the distribution of alcohol intake among men only; furthermore, the effect of exclusion was dissimilar for colon and rectal cancer. We do not have a ready explanation for these differences.

Drinking habits are strongly related to dietary habits and other lifestyle characteristics. The strong association between smoking and alcohol consumption is a very common finding. The association between alcohol and Quetelet index, which was shown to be positive in our male population just as in another Dutch population (26), is not found in all populations (27), e.g. in the USA in which almost no association has been demonstrated (28). In women, the slightly negative association corresponded with that reported in the USA (28). Level of education is strongly and positively related to alcohol intake. This association may be restricted to older age groups (29). The absence of a relation with fat intake and a negative relation with fiber intake has also been reported for other populations (30). A positive association of alcohol with protein from meat has also been shown (30). The finding that energy from alcohol did not substitute but is merely added to energy from other sources is consistent with many other studies (27). All characteristics described, including history of gallbladder surgery (31), were determinants of either colon or rectal cancer or both. Because of their strong associations with alcohol intake, we included them in all multivariate analyses. The results of the multivariate analysis showed, however, that the estimates of the relative rates for alcohol consumption were only slightly confounded. It is therefore unlikely that residual confounding due to potential inaccurate measurement of these confounders explains the association found between alcohol intake and rectal cancer. We cannot entirely exclude, of course, the possibility that another, unevaluated confounder is involved in this relation.

The consistent $U$-shaped dose-effect relation for alcohol intake and colon cancer may be considered to result from the choice of abstainers as reference category. The group of abstainers may comprise subjects with "deviant" characteristics and an increased risk for (colon) cancer. Nevertheless, we had a number of reasons to choose abstainers as sole reference instead of, for example, abstainers and very light drinkers combined. First, from the dose-response curves presented, the effect of the choice of a different reference group can be interpolated. This is not true if a combined reference group is used. Second, it is unlikely that the much larger, culturally determined, proportion of female abstainers comprised a similar proportion of "deviant" subjects as 
male abstainers. We nevertheless observed the same U-shaped relationship for women. Third, combining abstainers with the group having an intake of less than 5 gram ethanol per day would still show a U-shaped curve, since the lowest risk is associated with a consumption between 5 and $15 \mathrm{~g}$ of ethanol daily, corresponding to approximately one glass. U-shaped curves have also been reported in other studies. A case-control study in France (6) found the lowest RR of colon cancer for an alcohol intake of 10-15.5 ml per day in women; in men, for whom not any association was observed, alcohol intake was much higher. Stemmermann et al. (5) observed in a prospective study among Japanese in Hawaii the lowest risk at a slightly lower level of alcohol consumption. The monotonic significantly decreasing relative rate for colon cancer for increasing liquor consumption has not been reported in other studies.

Unlike the results of the meta-analysis (1), which averaged the results over ali studies included, our study showed a clearly increased risk for rectall cancer, whereas the risk of colon cancer tended to be reduced at moderate alcohol consumption levels. This result is in line with other studies reporting a higher risk for rectal than for colon cancer. Some of these studies were included in the meta-analysis $(3,4,32)$, while others have been published later $(6,7)$. Beer consumption is also implicated in an increased risk for rectal cancer in a substantial number of studies $(2,3,5,6,8,9)$. Cohort studies among brewery workers have provided evidence of an increased risk of rectal cancer among beẽ drinkers in Ireland (33) and Sweden (34), but not in Denmark (10). Correlation studies have also supported the relation between beer consumption and rectal cancer $(25,36)$. The study by Potter et al. (36) furthermore presented evidence that the sex ratio of rectal cancer mortality is higher than unity in beer drinking countries in contrast to countries where beer consumption is low. We have calculated from our data (Table 7) the number of cases attributable to beer drinking (37). The high proportion of male (57\%) compared to female $(9 \%)$ beer drinkers appeared to explain part of the difference in incidence of rectal cancer between men and women. In men, 25 of the 73 cases were attributable to beer consumption, and in women only 3 out of 33 .

The mechanism for the effect of alcohol on the development of colorectal cancer is not clear. Contrary to the upper digestive tract, which has direct contact with the ingested alcohol, the influence on the lower digestive tract must be indirect. One of the most plausible explanations is the effect of alcohol on liver enzymes, demonstrated in rats, which results in decreased "first-pass clearance" of carcinogens, in particular nitrosamines (38). Other explanations include the effect of ethanol on cell proliferation of rectal mucosa, possibly caused by the ethanol metabolite acetaldehyde (38). It may be that the consumption of alcohol increases risk due to a combined action of ethanol and contaminants in alcoholic beverages. This may also explain the differences between results of epidemiological studies.

It has been shown that beer in the Netherlands used to be contaminated with a relatively large amount (mean $1.2 \mu \mathrm{g} / \mathrm{kg}$ ) of $\mathrm{N}$-nitrosodimethylamine (NIDMA) (39). Beer accounted for $90 \%$ of NDMA intake of beer drinkers, whose intake of NDMA was ten times higher than that of non-beer drinkers. The contamination of beer with NDMA, which was caused by direct heating of the malt through gas-firing, was discovered in 1978. Subsequent changes in de production process of malt since 1979 decreased the contamination with NDMA to low levels (40). From other countries (e.g. Germany, New Zealand, USA) high NDMA levels in beer have also been reported (41, 42). If contamination of beer with nitrosamines has caused the increased risk for rectal cancer, we would expect a decrease in relative risk associated with beer drinking over time. It is likely that we have observed in our study, conducted eight to ten years after the change in beer production, a relative risk that is still influenced by beer consumption before 1979. Unfortunately, no data are available from earlier studies in 
the Netherlands. A direct proof of the role of nitrosamine contamination of beer in the development of rectal cancer in man may come from future studies in this country; we would expect the relative risk associated with the consumption of beer adjusted for alcohol to reduce.

If beer consumption is causal to the development of rectal cancer, we would expect an increasing risk with increasing dose. Such a dose-response relation, however, was not evident from our data. It is plausible to presume that the quantitative assessment is likely to refer more to the recent than to the distant past (i.e. more than ten years before baseline). If it is true that beer consumption did not entail a higher risk during the eight years immediately preceding baseline assessment, then the assessment may not have been sufficiently related to the quantitative intake of beer before that period, resulting in the absence of a dose-response relation.

In conclusion, alcohol consumption did not increase risk for colon cancer; light to moderate intake, i.e. less than two drinks per day, even implied a significantly decreased risk, which held for all types of alcoholic beverages. Risk of rectal cancer, on the contrary, is clearly increased for moderate to heavy alcohol consumption (more than 30 gram per day). The enhancing effect appeared to be mainly attributable to the consumption of beer. Light to moderate consumption of alcoholic beverages does not appear to be harmful with respect to overall risk of colorectal cancer, but rather tends to reduce risk. If the increased risk of rectal cancer by beer was caused by its high content of nitrosamines in the past, then this problem has been solved already. The data do not permit a conclusion about the risk of very heavy drinkers, since this category was insufficiently represented in the cohort.

\section{References}

1. Longnecker MP, Orza MJ, Adams: ME, Vioque J, Chalmers IC. A meta-analysis of alcoholic beverage consumpition in relation to risk of colorectal cancer. Cancer Causes and Control 1990; 1 : 59-68.

2. Williams R, Horm JW. Association of cancer sites with tobacco and alcohol consumption and socioeconomic status of patients: Interview study from the Third National Cancer Survey. J Natl Cancer Inst 1977; 58: 525-547.

3. Kune S, Kune GA, Watson LF. Case-control study of alcoholic beverages as etiological factors: the Melbourne Colorectal Cancer Study. Nutr Cancer. 1987; 9; 43-56.

4. Klatsky A , Armstrong, MA, Friedman GD, Hiatt RA. The relations of alcoholic beverage use to colon and rectal cancer. Am J Epidemiol 1988; 128: 1007-1015.

5. Stemmermann GN, Nomura AMY, Chyou P, Yoshizawa C. Prospective study of alcohol intake and large bowel cancer. Dig Dis and Sciences 1990; 35: 414-420.

6. Riboli E, Cornée J, Macquart-Moulin G, Kaaks R, Casagrande C, Guyader M. Cancei and polyps of the colorectum and lifetime consumption of beer and other alcoholic beverages. Am £ Epidemiol 1991: 134: 157-166.

7. Choi SY, Kahyo H. Effect of cigarette smoking and alcohol consumption in the etiology of cancers of the digestive tract. Int. J Cancer 1991; 49; 381-386.

8. Freudenheim JI, Graham S, Marshall JR, Haughey BP, Wilkinson G. Lifetime alcohol intake and risk of rectal cancer in western New York. Nutr Cancer 1990; 13: 101-109.

9. Kabat GC, Howson CP, Wynder EL. Beer consumption and rectal cancer. Int J Epidemiol 1986; 15: 494-501.

10. Jensen OM. Cancer morbidity and causes. of death among. Danish brewery workers. Int, J Cancer $1979 ; 23 ; 454-463$.

11. Wu AH, Paganini-Hill A, Ross, RK, Henderson BE. Alcohol, physical activity and other risk factors for colorectal cancer: a prospective study. Br J Cancer 1987; 55: 687-694.

12. Preussmann R. Occurrence and exposure to n-nitroso compounds and precursors. In: O'Neill IK, Von Borstel RC; Miller CT et al. (eds). N-nitroso compounds: occurrence, biological effects and relevance to human cancer. Lyon: IARC, 1984:3-15.

13. Van den Brandt PA, Goldbohm RA, Van 't Veer P, Volovics A, Hermus RJ, Sturmans F. A largescale prospective cohort study on diet and cancer in the Netherlands. J Clin Epidemiol 1990; 43: 285-295. 
14. Van den Brandt PA, Schouten LJ, Goldbohm RA, Dorant E, Hunen PMH. Development of a record linkage protocol for use in the Dutch cancer registry for epidemiological research. Int J Epidemiol 1990; 19: 553-558.

15. Goldbohm RA, Van den Brandt PA, Dorant E. Estimation of the coverage of municipalities by: cancer registries and PAI.GA using hospital discharge data (submitted for publication).

16. Goldbohm RA, Van den Brandt PA, Brants HAM et al. Validation of a dietary questionnaire used in large-scale prospective cohort study on diet and cancer (submitted for publication).

17. NEVO tabel. Dutch food composition table 1986-1987. The Hague, the Netherlands: Voorlichtingsbureau voor de Voeding, 1986.

18. Willeı W, Stampfer M. Total energy intake: implications for epidemiologic analyses. Am J Epidemiol 1986; 124: 17-27.

19. Self SG, Prentice RL. Asymptotic distribution theory and efficiency results for case-cohort studies. Ann Stat 1988; 16; 64-81.

20. Volovics A, Van den Brandt PA. Stratified and simple regression methods for the analysis of casecohort studies (submitted for publication).

21. Willett WC. Reproducibility and validity of food-frequency questionnaires. In: Willett WC, ed. Nutritional Epidemiology. New York/Oxford: Oxford University Press, 1990; 92-126.

22. Goldbohm RA, Van den Brandt PA, Van 't Veer P et al. Reproducibility of a food frequency questionnaire and stability of dietary habits determined from five annually repeated measurements. (submitted for publication).

23. Jensen OM, Wahrendorf J, Rosenqvist A, Geser A. The reliability of questionnaire-derived historical dietary information and temporal stability of food habits in individuals. Am $\mathrm{J}$ Epidemiol 1984: 120: 281-290.

24. Byers TE, Rosenthal RI, Marshall JR, Rzepka TF, Cummings. KM, Graham S. Dietary history from the distanı past: a methodological study. Nutr Canc 1983; 5: 69-77.

25. Wu MI, Whittemore AS, Jung DI Errors in reported dietary intakes, II. Long-term recall. Am J Epidemiol 1988; 128: 1137-1145.

26. Kromhout D. Energy and macronutrient intake in lean and obese middle-aged men (the Zutphen Study). Am J Clin Nutr 1983; 37: 295-299.

27. Hellerstedt WI. Jeffery RW, Murray DM. The association between alcohol intake and adiposity in the general population. Am J Epidemiol 1990; 132: 594-611

28. Colditz GA, Giovannucci E, Rimm EB et al. Alcohol intake in relation to diet and obesity in women and men. An J Clin Nutr 1991: 54: 49-55.

29. Romelsjo A. The relationship between alcohol comsumption and social status in Stockholm. Has the social pattern of aleohol comsumption changed? Ini J Epidemiol 1989; 18: 842-851.

30. Herbeth B, Didelot-Bartheleny $I_{2}$ Lenoine $A$, le Devehat $C$. Dietary behavior of French men according to alcohol drinking pattern. I Stud Alcohol 1988; 49; 268-272.

31. Goldbohm RA, Van den Brandi PA, Van 'I Veer P, Dorant E, Sturmans F, Hermus RJJ. Cholecystectomy and colorectal cancer: evidence from a cohort study on diet and cancer. Ini J Cancer (in press).

32. Pollack ES, Nomura AM, Meilbrun LK, Stemmermann GN, Green SB. Prospective study of alcohol consumption and cancer. N Engl J Med 1984; 310:617-621.

33. Dean G, Maclennan R, Mcloughlin H, Shelley E. Causes of death of blue-eollar workers at a Dublin brewery, 1954 - 73. Br J Cancer 1979; 40: 581-589.

34. Carstensen JM. Hygren LO, Hatschek T. Cancer incidence among Swedish brewery workers. Int J Cancer 1990; 45: 393-396.

35. Enstrom JE. Colorectal cancer and beer drinking. Br J Cancer 1977; 35: 674-683.

36. Potter ID, McMichael N. Hartshorne JM. Alcohol and beer consumption in relation to cancers of bowel and lung: an extended correlation analysis. M Chron Dis 1982; 35: 833-842.

37. Kleinbaum DG, Kupper I.I, Morgenstern H. Epidemiologic research: Principles and quantitative methods. London: Lifetime Learning Publications 1982.

38. Seitz HK, Simanowski UA. Alcohol and carcinogenesis. Ann Rev Nutr 1988; 8: 99-119.

39. Stephany RW, Schuller PL. Daily dietary intakes of nitrate, nitrite and volatile n-nitrosamines in the Netherlands using the duplicate portion sampling technique. Oncology 1980; 37: 203-210.

40. Fllen G, Schuller PI. N-nitrosamine investigations in the Netherlands: highlights from the last ten years. In: Preussman R (ed). Das Nitrosamin-Problem. Weinheim Verlag 1983; 81-92.

41. Spiegelhalder B, Fisenbrand G, Preussmann R. Contamination of beer with trace quantities of Nnitrosodimethylamine. Fd Cosmet Toxicol 1979; 17:29-31.

42. Weston RJ. N-nitrosamine content of New Zealand beer and malted barley. J Scri Food Agric 1983; 34: $1005-1010$. 


\title{
Chapter 13
}

\section{A prospective cohort study on the relation between meat consumption and the risk of colon cancer*}

\section{R. Alexandra Goldbohm ${ }^{1,2}$, Piet A. van den Brandt ${ }^{2}$, Pieter van't Veer ${ }^{1}$,} Henny A.M. Brants ${ }^{1}$, Elisabeth Dorant ${ }^{2}$, Ferd Sturmans ${ }^{2}$, Rudolph J.J. Hermus ${ }^{1}$.

1) TNO Toxicology and Nutrition Institute, Zeist, the Netherlands; 2) University of Limburg, Department of Epidemiology, Maastricht, the Netherlands

\begin{abstract}
The association between the consumption of meat and cancer of the colon was investigated in a prospective cohort study on diet and cancer, which is being conducted in the Netherlands since 1986 among 120,852 men and women, aged 55-69. The analysis was based on 215 incident cases of colon cancer (105 men and 110 women) accuinulated In 3.3 years of follow-up, excluding cases dlagnosed In the first year of follow-up. No trends in relative rates were detected for intake of energy and energyadjusted intake of fats, protein, fat from meat and protein from meat. Consumption of (fresh) meat, beef, pork, minced meat, chicken and nsh was not assoclated with risk of colon cancer either. Meat products, however, were shown to Increase risk In men and women (RR 1.17 per 15 g/day, 95\% confidence interval 1.03-1.33).
\end{abstract}




\section{Introduction}

A number of articles have reviewed the epidemiologic evidence for an association between dietary habits and the risk of colon cancer (1-4). Although consensus as to a protective effect of dietary fiber seems to develop, debate remains about the relation between meat consumption and/or fat intake and colon cancer. In case-control studies, positive associations with meat consumption or with fat intake have been found frequently, but the majority of the studies conducted yielded non-significant results $(5,6)$. Few results are available from prospective studies, which may carry more weight than case-control studies in assessing the relation between diet and cancer since they are presumed not to be biased by recall of past dietary habits after the cancer has been diagnosed. All but two prospective studies were conducted in the USA. Bjelke (7) found an increased relative risk for processed meat only (Norway, 65 cases). Hirayama (8) observed an increased risk of colon cancer with frequency of meat consumption in the group with infrequent vegetable consumption among a cohort of 265,000 men and women in Japan. Phillips and Snowdon (9) did not find a clear gradient in risk for frequency of meat and poultry consumption in a population of Seventh Day Adventists (139 cases), which included a large proportion of vegetarians. A prospective study among Hawaiian Japanese men (102 cases, 10) found a negative association with fiber intake, but no association with meat and fat consumption. A more recent analysis of those data, including more cases (182), showed a negative tendency for the association with fat intake (11). A publication on the Nurses' Health Study, a prospective study among female nurses, showed an inereased risk of colon cancer (150 cases) for the consumption of meat, in particular beef, pork and lamb, and also for the intake of fat, in particular saturated and monounsaturated fat (12). Quite surprisingly, the association between animal protein and the risk of colon cancer was found to be slightly inverse in this study. A comparable prospective study among middle-aged women, using a similar, although extended, dietary questionnaire, did not find an association of colon cancer (158 cases) with fat nor with fiber intake (13). In the large Cancer Prevention Study II (1150 fatal cases) no association with meat consumption or fat intake was observed (14).

We have studied the relation between meat consumption and the risk of colon cancer in a prospective cohort study on diet and cancer that was initiated in the Netherlands in 1986. Apart from meat consumption, we also included fat and protein in the analysis to obtain better insight into the origin of a possibly increased risk. Consumption of fish was included since it may substitute meat consumption.

\section{Subjects and methods}

\section{The cohort}

The prospective cohort study on diet and cancer has been initiated in the Netherlands in September 1986. The cohort included 58,279 men and 62,573 women aged 55-69 at the start of the study. The study population originated from 204 municipal population registries throughout the country. At baseline, the cohort members completed a mailed, self-administered questionnaire on dietary habits and other risk factors for cancer. For data processing and analysis the case-cohort approach was used: the cases were enumerated for the entire cohort, while the person years at risk accumulating in the cohort were estimated from a random sample (subcohort). This subcohort of 3500 subjects ( 1688 men, 1812 women) was sampled from the cohort after baseline measurement and was followed up for vital status over $\mathbf{3 . 3}$ years. The study design has been described in detail elsewhere (15). 


\section{Follow-up for cancer}

Follow-up for incident cancer was established by computerized record linkage with all nine regional cancer registries in the Netherlands and with PALGA, the Dutch national data base of pathology reports. The method of record linkage has been published previously (16). The present analysis is restricted to cancer incidence in the period from September 1986 (baseline measurement) to December 1989, i.e. a followup period of 3.3 years. In this period, completeness of follow-up of the cohort through linkage with the cancer registries and PALGA was estimated to be $95 \%$ (17). After excluding subjects who reported a history of cancer other than skin cancer in the baseline questionnaire, a total of 312 incident cases with microscopically confirmed primary adenocarcinoma of the colon (i.e. cecum through sigmoid colon) were identified (157 men and 155 women).

\section{Questionnaire}

The self-administered questionnaire has been described in more detail elsewhere (17). For the present analysis, characteristics of interest are summarized below. The dietary section of the questionnaire, a 150 -item semi-quantitative food frequency questionnaire, concentrated on habitual intake of food and beverages during the year preceding the start of the study. The questionnaire contained 14 items on the consumption of meat with the hot meal (mainly fresh meat, including chicken), 5 items on the consumption of meat products, which are used as sandwich filling, and 3 items on fish consumption. As for the serving sizes, a question was included on the quantity of (fresh) meat usually purchased (per person, per meal). For meat products, the number of sandwiches filled with a product was asked. For chicken and fish standard serving sizes were used.

Daily mean nutrient intakes were calculated using the computerized Dutch food composition table (18). Energy adjustment of nutrient intakes was done by regression analysis according to Willett and Stampfer (19). The questionnaire was validated against a 9-day diet record (Goldbohm et al, submitted for publication). The Pearson correlation coefficients between the mean daily intakes of energy, protein, fat, and fiber as assessed by the questionnaire and those estimated from the 9-day record were 0.70 , $0.61,0.72$ and 0.74 respectively; the corresponding energy- and sex-adjusted correlation coefficients were $0.59,0.52$ and 0.74 . The Spearman correlation coefficients for meat, meat products and fish were $0.46,0.54$ and 0.53 respectively.

\section{Data analysis}

Questionnaire data of all 312 cases and the subcohort were key-entered twice and processed in a manner blinded with respect to case/cohort status in order to minimize observer bias in coding and interpretation of the data. After excluding prevalent cancer cases other than skin cancer from the subcohort, 3346 subjects (1630 men and 1716 women) remained in this group. Furthermore, subjects with incomplete or inconsistent dietary data, according to criteria described by van den Brandt et al. (17), were excluded $(7.0 \%)$. Eventually, 150 male and 143 female colon cancer cases and 1525 male and 1598 female subcohort members were included in the analysis.

Fats and types of fat, protein as well as animal fat and animal protein (the latter two excluding fat and protein from dairy sources and margarine) were separately evaluated. Furthermore, daily mean consumption of the following food groups was included in the analysis: beef, pork, minced meat (mixed beef and pork), meat products (i.e. raw and cooked cured meat products and sausages), chicken and fish. Some of these food groups resulted from collapsing several items from the questionnaire. Variables were initially included as quintile categorical variables, except fish and meat 
products, which were classified into a non-user and three user categories $(0-10,10-20$, $\geq 20 \mathrm{~g} /$ day). Specific types of meat and meat products were only included as continuous variables in models that compared overall consumption with that decomposed in types. Age, dietary fiber intake and Quetelet index $\left(\mathrm{kg} / \mathrm{m}^{2}\right)$ were considered as potential confounders.

Data were analysed using the case-cohort approach (20), assuming exponentially distributed survival times in the follow-up period. Since standard software was not available for this type of analysis, specific programs were developed to account. for the additional variance introduced by sampling from the cohort instead of using the entire cohort (17). Since subclinical symptoms of large-bowel cancer may influence dietary habits before diagnosis, we excluded cases in the first year of follow-up after assessing the mean intake of cases diagnosed in different follow-up years.

\section{Results}

Table 1 presents mean daily intake of the variables most relevant to this analysis for subcohort and cases categorized according to year of diagnosis. Among men, energy intake was lower in cases diagnosed in the first year of follow-up, but energy-adjusted fat intake remained fairly constant. Among, women, no appreciable difference in absolute intake was detected, but energy-adjusted intake of fat and protein appeared to be lower in the cases diagnosed in the first year of follow-up. None of these differences, however, reached statistical significance. Subsequent analyses excluded cases diagnosed in the first year.

Table 1. Energy, fat and protein intake in the subcohort and colon cancer cases according to year of diagnosis.

\begin{tabular}{|c|c|c|c|c|c|c|c|}
\hline \multirow[b]{2}{*}{ Nutrient } & \multirow[b]{2}{*}{ Year of diagnosis } & \multicolumn{3}{|l|}{ Men } & \multicolumn{3}{|c|}{ Women } \\
\hline & & $\mathrm{n}$ & Mean & SD & $\mathrm{n}$ & Mean & SD \\
\hline \multicolumn{8}{|l|}{ Energy (kcal)* } \\
\hline Subcohort & - & 1519 & 2.159 & 509 & 1592 & 1688 & 409 \\
\hline \multirow{3}{*}{ Cases } & 1 & 45 & 1930 & 419 & 33 & 1723 & 516 \\
\hline & 2 & 46 & 2194 & 435 & 38 & 1592 & 390 \\
\hline & $\geq 3$ & 59 & 2072 & 436 & 72 & 1673 & 378 \\
\hline \multicolumn{8}{|l|}{ Fat $(g) \dagger$} \\
\hline Subcohort & $=$ & 1519 & 93.7 & 14.4 & 1592 & 74.2 & 10.5 \\
\hline \multirow{3}{*}{ Cases } & 1 & 45 & 93.0 & 15.2 & 33 & 71.6 & 8.6 \\
\hline & 2 & 46 & 93.3 & 125 & 38 & 72.8 & 10.7 \\
\hline & $\geq 3$ & 59 & 94.9 & 13.0 & 72 & 75.5 & 113 \\
\hline \multicolumn{8}{|l|}{ Protein $(\mathrm{g}) \dagger$} \\
\hline Subcohort & - & 1519 & 75.4 & 11.4 & 1592 & 65.7 & 10.6 \\
\hline \multirow{3}{*}{ Cases } & 1 & 45 & 72.1 & 10.2 & 33 & 64.3 & 9.0 \\
\hline & 2 & 46 & 75.7 & 8.6 & 38 & 66.2 & 10.6 \\
\hline & $\geq 3$ & 59 & 74.0 & 9.6 & 72 & 65.5 & 12.0 \\
\hline \multicolumn{8}{|l|}{ Meat fat (g)†‡ } \\
\hline Sulcohort & - & 1519 & 19.9 & 8.4 & 1592 & 15.8 & 7.2 \\
\hline \multirow{3}{*}{ Cases } & 1 & 45 & 20.1 & 6.6 & 33 & 14.0 & 6.5 \\
\hline & 2 & 46 & 20.1 & 8.6 & 38 & 15.0 & 8.1 \\
\hline & $\geq 3$ & 59 & 20.4 & 8.6 & 72 & 15.4 & 8.1 \\
\hline \multicolumn{8}{|c|}{ Meat protein (g) †f } \\
\hline Subcohort & - & 1519 & 28.0 & 9.6 & 1592 & 24.0 & 9.0 \\
\hline \multirow{3}{*}{ Cases } & 1 & 45 & 28.9 & 9.5 & 33 & 21.7 & 8.5 \\
\hline & 2 & 46 & 27.5 & 8.2 & 38 & 24.0 & 8.6 \\
\hline & $\geq 3$ & 59 & 27.7 & 103 & 72 & 23.5 & 9.1 \\
\hline
\end{tabular}

- Age-adjusted; + Age- and energy-adjusted; $¥$ Meat fat and protein: animal fat and protein excluding dairy sources and margarine. 
Table 2. Sex-adjusted Pearson correlation coefficients between meat types and intake of energy and energy-adjusted fats, protein and dietary fiber in the subcohort.

Fresh meat

$\begin{array}{lrrrrrrr}\text { Nutrient } & \text { Total } & \text { Beef } & \text { Pork } & \text { Minced } & \text { Chicken } & \text { Fish } & \text { Meat Prov } \\ & & & & & & & \\ & & & & & & & \\ \text { Energy } & 0.21 & 0.09 & 0.13 & 0.05 & 0.03 & 0.10 & 0.32 \\ \text { Fat } & 0.21 & 0.03 & 0.19 & 0.15 & 0.00 & -0.06 & 0.03 \\ \quad \text { Saturated } & 0.15 & 0.08 & 0.11 & 0.14 & -0.06 & -0.10 & 0.01 \\ \quad \text { Monounsaturated } & 0.33 & 0.05 & 0.28 & 0.21 & 0.03 & -0.06 & 0.08 \\ \quad \text { Polyunsaturated } & -0.03 & -0.08 & 0.01 & -0.03 & 0.06 & 0.05 & 0.02 \\ \text { Protein } & 0.46 & 0.24 & 0.24 & 0.18 & 0.20 & 0.22 & 0.22 \\ \text { Meat fat } & 0.67 & 0.15 & 0.59 & 0.44 & -0.08 & -0.07 & 0.40 \\ \text { Meat protein } & 0.84 & 0.39 & 0.53 & 0.27 & 0.28 & 0.31 & 0.38 \\ \text { Dietary fiber } & -0.18 & -0.05 & -0.17 & -0.06 & 0.01 & 0.01 & -0.05\end{array}$

- Composed of beef and pork

Table 2 gives the Pearson correlation coefficients for meats with energy intake and energy-adjusted intakes of fats, protein and dietary fiber. Meat consumption was not strongly correlated with energy intake. The relatively high correlation of meat products with energy could be explained by the asscciation of bread consumption with energy. Consumption of pork appeared to contribute most to the intake of fat, in particular monounsaturated fat. The consumption of meat and meat products was positively associated ( $\mathrm{r}: 0.14)$, whereas meat and fish consumption were not related. Consumption of chicken correlated negatively with other types of meat ( $\mathrm{r}$ : -0.05 to $-\mathbf{0 . 1 3}$ ).

Table 3 displays the relative rates (RR) of colon cancer for energy intake and energy-adjusted intakes of fats and protein. None of the variables showed any evidence of a (positive or negative) trend across quintiles of intake. For fat and protein derived from meat no trend was detected either (Table 4). In Table 4, the pooled estimates for men and women, which were also adjusted for dietary fiber intake, did not show any association with risk either.

Table 5 shows the relative rates for the consumption of meat (including chicken), meat products and fish. These data were adjusted for energy intake by including energy in the multivariate model. The results for meat were consistent with those from Table 4, i.e. no evidence of a trend. Similar results were seen for frequency of meat consumption. The RRs were $0.65,0.56,0.78$, and 0.81 for consumption frequencies of $3 / 4,5,6$ and 7 days per week respectively, relative to the reference group using meat on 0-2 days per week. Consumption of meat products, however, showed a (non-significant) positive trend in men $(p=0.06)$ and women $(p=0.10)$.

For fish consumption a weakly negative, but not significant association with colon cancer occurrence was observed. Pooled results for men and women in the table were also adjusted for dietary fiber intake, which had a small effect on the estimates. As was to be expected, only meat products showed a significant $(\mathrm{p}=0.017)$ positive trend. When fitted as a continuous variable, this resulted in a RR of 1.17 (95\% CI 1.03-1.33) for an increment of $15 \mathrm{~g}$ (equivalent to one sandwich filling) of mean daily consumption of meat products. Introduction of fat from meat into the models for meat and meat products did not have any effect on the estimates for meat, but strengthened the association between meat products and colon cancer $(p=0.007)$. Addition of the 
Quetelet index, which was positively associated with meat consumption, to the models did not change the estimates.

Table 3. Age-adjusted relative rates (RR) for quintiles of energy and energy-adjusted nutrient. intakes.

\begin{tabular}{|c|c|c|c|c|c|c|c|c|}
\hline \multirow{2}{*}{$\begin{array}{l}\text { Nutrient } \\
\text { quintile }\end{array}$} & \multicolumn{3}{|l|}{ Men } & \multicolumn{3}{|l|}{ Women } & \multicolumn{2}{|c|}{ Both sexes } \\
\hline & Median $^{*}$ n $\dagger$ & RR & $95 \% \mathrm{CI}$ & Median* ${ }^{*} \dagger$ & RR & $95 \% \mathrm{CI}$ & $\mathrm{RR} \ddagger$ & $95 \% \mathrm{Cl}$ \\
\hline
\end{tabular}

Energy (kcal)

\begin{tabular}{|c|c|c|c|c|c|c|c|c|c|c|}
\hline & & & & & & & & & & \\
\hline 1 & 1510 & 23 & 1.00 & $=$ & 1163 & 25 & 1.00 & - & 1.00 & - \\
\hline 2 & 1836 & 21 & 0.92 & $0.50-1.70$ & 1435 & 21 & 0.85 & $0.47-1.55$ & 0.88 & $0.67 \cdot 1.14$ \\
\hline 3 & 2096 & 23 & 1.02 & $0.56-1.86$ & 1626 & 31 & 1.22 & $0.70-2.12$ & 1.12 & $0.87-1.46$ \\
\hline 4 & 2364 & 24 & 1.09 & $0.60-1.98$ & 1848 & 15 & 0.62 & $0.32-1.20$ & 0.84 & $0.54-1.31$ \\
\hline 5 & 2791 & 14 & 0.72 & $0.36-1.45$ & 2200 & 18 & 0.75 & $0.40-1.41$ & 0.74 & $0.39-1.39$ \\
\hline & & & & & & & & & & \\
\hline
\end{tabular}

\begin{tabular}{|c|c|c|c|c|c|c|c|c|c|c|}
\hline \multicolumn{11}{|c|}{ Fat $(\mathrm{g})$} \\
\hline 1 & 76 & 20 & 1.00 & - & 61 & 24 & 1.00 & - & 1.00 & - \\
\hline 2 & 87 & 22 & 1.14 & $0.61-2.13$ & 69 & 19 & 0.79 & $0.42-1.47$ & 0.90 & $0.57-1.41$ \\
\hline 3 & 94 & 18 & 0.87 & $0.45-1.67$ & 74 & 17 & 0.72 & $0.38-1.36$ & 0.74 & $0.46-1.18$ \\
\hline 4 & 100 & 23 & 1.11 & $0.60-2.07$ & 79 & 22 & 0.91 & $0.50-1.67$ & 0.94 & $0.60-1.46$ \\
\hline 5 & 111 & 22 & 1.10 & $0.59-2.07$ & 87 & 28 & 1.13 & $0.64-2.00$ & 1.07 & $0.70-1.64$ \\
\hline D-va & trens & & & & & & & & & \\
\hline
\end{tabular}

Saturated fat (g)

\begin{tabular}{ccccccccccc}
1 & 28 & 21 & 1.00 & & 23 & 21 & 1.00 & & 1.00 & \\
2 & 32 & 17 & 0.79 & $0.41-1.52$ & 27 & 23 & 1.10 & $0.59-2.02$ & 0.88 & $0.56-1.40$ \\
3 & 36 & 27 & 1.23 & $0.68-2.23$ & 29 & 18 & 0.85 & $0.45-1.63$ & 0.97 & $0.62-1.52$ \\
4 & 40 & 20 & 0.90 & $0.47-1.69$ & 32 & 17 & 0.79 & $0.41-1.53$ & 0.77 & $0.48-1.23$ \\
5 & 47 & 20 & 0.90 & $0.47-1.70$ & 37 & 31 & 1.36 & $0.77-2.42$ & 1.07 & $0.69-1.66$ \\
p-value for trend & \multicolumn{3}{c}{0.882} & & & & 0.511 & & 0.914
\end{tabular}

Monounsaturated fat $(\mathrm{g})$

\begin{tabular}{ccccccccccc}
1 & 27 & 21 & 1.00 & & 22 & 20 & 1.00 & - & 1.00 & - \\
2 & 32 & 18 & 0.91 & $0.47-1.75$ & 25 & 25 & 1.19 & $0.65-2.19$ & 0.98 & $0.62-1.53$ \\
3 & 35 & 21 & 1.03 & $0.55-1.93$ & 27 & 24 & 1.15 & $0.62-2.14$ & 1.01 & $0.64-1.59$ \\
4 & 38 & 20 & 0.94 & $0.50-1.77$ & 30 & 23 & 1.10 & $0.59-2.05$ & 0.91 & $0.58-1.44$ \\
5 & 43 & 25 & 1.26 & $0.69-2.31$ & 33 & 18 & 0.88 & $0.45-1.69$ & 1.00 & $0.63-1.57$ \\
p-value for trend & \multicolumn{3}{c}{0.453} & & & & 0.628 & & 0.882
\end{tabular}

Polyunsaturated fat $(\mathrm{g})$

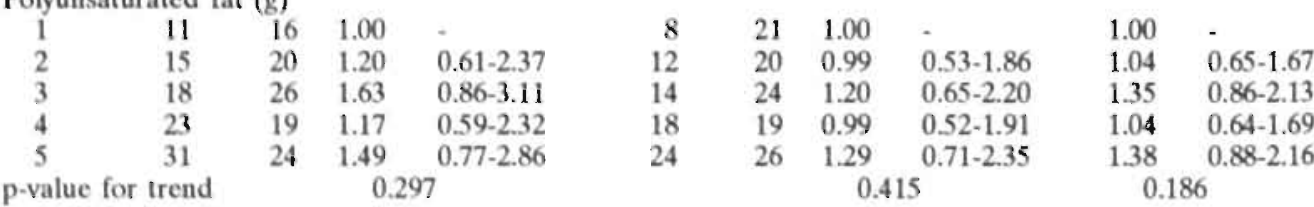

\begin{tabular}{|c|c|c|c|c|c|c|c|c|c|c|}
\hline \multicolumn{11}{|c|}{ Protein (g) } \\
\hline 1 & $6 !$ & 19 & 1.00 & - & 53 & 23 & 1.00 & - & 1.00 & - \\
\hline 2 & 69 & 24 & 1.36 & $0.72-2.56$ & 60 & 20 & 0.88 & $0.47-1.63$ & 1.10 & $0.70-1.71$ \\
\hline 3 & 75 & 25 & 1.37 & $0.74-2.55$ & 65 & 18 & 0.80 & $0.42-1.50$ & 1.05 & $0.67-1.65$ \\
\hline 4 & 81 & 25 & 1.53 & $0.82-2.87$ & 70 & 25 & 1.09 & $0.61-1.96$ & 1.28 & $0.82-2.00$ \\
\hline 5 & 90 & 12 & 0.67 & $0.32-1.43$ & 79 & 24 & 1.05 & $0.58-1.89$ & 0.90 & $0.57-1.42$ \\
\hline \multicolumn{2}{|c|}{ p-value for trend } & \multicolumn{3}{|c|}{0.553} & & \multicolumn{3}{|c|}{0.634} & \multicolumn{2}{|c|}{0.953} \\
\hline
\end{tabular}

- Median of energy or nutrient intake in the quintile.

$\dagger$ Number of colon cancer cases in the quintile.

$\ddagger$ RR also adjusted for sex and dietary fiber intake. 
Table 4. Age-adjusted relative rates (RR) for quintiles of energy-adjusted intake of fat and protein derived from meat.

\begin{tabular}{|c|c|c|c|c|c|c|c|c|c|c|}
\hline \multirow{2}{*}{$\begin{array}{l}\text { Nutrient } \\
\text { quintile }\end{array}$} & \multicolumn{4}{|l|}{ Men } & \multicolumn{4}{|l|}{ Women } & \multicolumn{2}{|c|}{ Both sexes } \\
\hline & Median & n & RR & $95 \% \mathrm{CI}$ & Median & $\mathrm{n}$ & RR & $95 \% \mathrm{CI}$ & $\mathbf{R R}^{*}$ & $95 \% \mathrm{CI}$ \\
\hline \multicolumn{11}{|c|}{ Meat fat (g) } \\
\hline 1 & 10 & 24 & 1.00 & - & 7 & 24 & 1.00 & - & 1.00 & . \\
\hline 2 & 16 & 18 & 0.75 & $0.40-1.42$ & 12 & 29 & 1.22 & $0.70-2.15$ & 0.95 & $0.62 \cdot 1.45$ \\
\hline 3 & 19 & 20 & 0.86 & $0.46-1.59$ & 15 & 13 & 0.56 & $0.28-1.12$ & 0.66 & $0.42-1.05$ \\
\hline 4 & 23 & 17 & 0.73 & $0.38-1.38$ & 19 & 22 & 0.90 & $0.49 \cdot 1.64$ & 0.76 & $0.49 \cdot 1.19$ \\
\hline 5 & 31 & 26 & 1.13 & $0.63-2.02$ & 25 & 22 & 0.94 & $0.52-1.72$ & 0.98 & $0.64-1.49$ \\
\hline \multicolumn{2}{|c|}{ p-value for trend } & \multicolumn{3}{|c|}{0.724} & & & \multicolumn{2}{|c|}{0.474} & \multicolumn{2}{|c|}{0.668} \\
\hline \multicolumn{11}{|c|}{ Meat protein $(\mathrm{g})$} \\
\hline 1 & 17 & 21 & 1.00 & - & 13 & 20 & 1.00 & . & 1.00 & . \\
\hline 2 & 23 & 26 & 1.18 & $0.65-2.15$ & 20 & 24 & 1.21 & $0.65-2.24$ & 1.16 & $0.75-1.78$ \\
\hline 3 & 27 & 18 & 0.87 & $0.45-1.67$ & 24 & 22 & 1.05 & $0.56-1.97$ & 0.91 & $0.58-1.44$ \\
\hline 4 & 32 & 20 & 0.94 & $0.50-1.77$ & 28 & 19 & 0.94 & $0.49-1.79$ & 0.90 & $0.57 \cdot 1.42$ \\
\hline 5 & 41 & 20 & 1.00 & $0.52-1.90$ & 35 & 25 & 1.24 & $0.68-2.29$ & 1.07 & $0.69-1.67$ \\
\hline \multicolumn{3}{|c|}{ p-value for trend } & \multicolumn{2}{|c|}{0.714} & & & \multicolumn{2}{|c|}{0.769} & \multicolumn{2}{|c|}{0.792} \\
\hline
\end{tabular}

- RR also adjusted for sex and dietary fiber intake.

Table 5. Relative rates (RR) for meat, meat products and fish consumption.

\begin{tabular}{|c|c|c|c|c|c|c|c|c|c|c|}
\hline \multirow{2}{*}{$\begin{array}{l}\text { Food } \\
\text { group }\end{array}$} & \multicolumn{4}{|l|}{ Men } & \multicolumn{4}{|l|}{ Women } & \multicolumn{2}{|c|}{ Both sexes } \\
\hline & Median & $\mathrm{n}$ & RR* & $95 \% \mathrm{CI}$ & Median & $n$ & $\mathrm{RR}^{*}$ & $95 \% \mathrm{CI}$ & $\mathrm{RR} \dagger$ & $95 \% \mathrm{CI}$ \\
\hline \multicolumn{11}{|c|}{ Meat $(\mathrm{g}) \neq$} \\
\hline 1 & 54 & 20 & 1.00 & - & 43 & 24 & 1.00 & - $\quad-1$ & 1.00 & - \\
\hline 2 & 84 & 22 & 1.09 & $0.58-2.04$ & 72 & 19 & 0.83 & $0.44-1.56$ & 0.92 & $0.59-1.44$ \\
\hline 3 & 101 & 30 & 1.62 & $0.89-2.93$ & 91 & 26 & 1.03 & $0.58-1.84$ & 1.24 & $0.81-1.90$ \\
\hline 4 & 123 & 18 & 0.98 & $0.51-1.91$ & 107 & 22 & 1.05 & $0.57-1.93$ & 0.98 & $0.62-1.55$ \\
\hline 5 & 158 & 15 & 0.87 & $0.43-1.77$ & 145 & 19 & 0.88 & $0.45-1.69$ & 0.84 & $0.51-1.37$ \\
\hline $\mathrm{p}$-value & r trend & \multicolumn{3}{|c|}{0.704} & & & \multicolumn{2}{|c|}{0.969} & \multicolumn{2}{|c|}{0.618} \\
\hline \multicolumn{11}{|c|}{ Meat products (g) } \\
\hline 0 & & 9 & 1.00 & - & & 14 & 1.00 & - & 1.00 & - \\
\hline $0-10$ & & 30 & 1.25 & $0.59-2.70$ & & 44 & 1.22 & $0.66-2.26$ & 1.23 & $0.76-1.98$ \\
\hline $10-20$ & & 29 & 1.45 & $0.67-3.12$ & & 30 & 1.48 & $0.77-2.87$ & 1.43 & $0.87-2.35$ \\
\hline$\geq 20$ & & 37 & 1.84 & $0.85-3.95$ & & 22 & 1.66 & $0.82-3.35$ & 1.72 & $1.03-2.87$ \\
\hline \multicolumn{2}{|c|}{ p-value for trend } & \multicolumn{3}{|c|}{0.061} & & & \multicolumn{2}{|c|}{0.097} & \multicolumn{2}{|c|}{0.017} \\
\hline \multicolumn{11}{|l|}{ Fish (g) } \\
\hline 0 & & 34 & 1.00 & - & & 36 & 1.00 & . & 1.00 & . \\
\hline $0-10$ & & 28 & 0.84 & $0.50-1.42$ & & 25 & 1.14 & $0.67-1.94$ & 1.00 & $0.68-1.47$ \\
\hline $10-20$ & & 11 & 0.41 & $0.21-0.83$ & & 22 & 1.14 & $0.66-1.97$ & 0.74 & $0.48-1.15$ \\
\hline$\geq 20$ & & 32 & 0.73 & $0.44-1.21$ & & 27 & 0.87 & $0.52-1.45$ & 0.81 & $0.56-1.17$ \\
\hline \multicolumn{2}{|c|}{ p-value for trend } & \multicolumn{3}{|c|}{0.093} & & & \multicolumn{2}{|c|}{0.635} & \multicolumn{2}{|c|}{0.139} \\
\hline
\end{tabular}

- Age (year) and energy included in model as continuous variables.

$\dagger$ RR also adjusted for sex. and dietary fiber intake.

$\ddagger$ Including all types of meat (except meat products) and chicken. 
The results of a further subdivision of meat and meat products are shown in Table 6, which displays the RRs for an increment in consumption of $15 \mathrm{~g} /$ day. For (fresh) meat, none of the results for types of meat deviated from those for total meat. For meat products, however, "other meat products", which mainly represented sausages, appeared to contribute most to the elevated RR.

Table 6. Mean, standard deviation and age- and energy-adjusted relative rate for types of meat and meat products, fitted as continuous variables.

Type (g/day)

Model*

Mean

SD

RR†

$95 \% \mathrm{CI}$

All (fresh) meat

Beef

Pork

Minced meat $\ddagger$

Liver

Chicken

Other meat

All meat products

Ham

Bacon§

I ean meat products I

Cooked liver

Other meat productsfl

$\begin{array}{ll}\text { a } & \\ \mathrm{b} & 29 \\ \mathrm{~b} & 38 \\ \mathrm{~b} & 18 \\ \mathrm{~b} & \\ \mathrm{~b} & \end{array}$

99

25

38

18

2

14

3

3

42.

0.98

0.96

0.99

0.91

0.90

1.03

0.99

6

0.99

0.93-1.03

$0.87-1.06$

$0.92-1.06$

0.80-1.04

0.54-1.48

$0.90-1.17$

0.66-1.47

1.03-1.33

1.17

1.04

$0.78-1.39$

1.25

$0.84 \cdot 1.88$

1.14

0.15

1.27

$0.82-1.61$

$0.02-1.12$

1.04-1.55

- Models were fitted for: (a) total (fresh) meat; (b) (fresh) meat decomposed in types; (c) total meat products; (d) meat products decomposed in types; all models were adjusted for sex, age and energy. L.R $-x^{2}$ for the (combined) meat terms: $0.99,3.33,5.77,11.13$ for models a to d respectively.

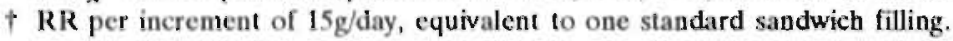

¥ Composed of beef and pork.

$\$$ Raw cured belly and dried backs.

II Raw cured smoked beef, lean cooked ham, leañ cooked pork.

9. Mainly sausages.

\section{Discussion}

We have presented evidence from a prospective study that the consumption of meat, fat from meat or protein from meat is not associated with an increased risk for colon cancer. The consumption of meat products, in contrast, appears to be consistently and positively related to risk for colon cancer.

After excluding the cases diagnosed in the first year of follow-up, this study included 215 colon cancer cases, indicating that it had reasonable but not very large power. We have thus to take into consideration that existing associations may not have been detected only because of insufficient power. Furthermore, the validity of the FFQ with respect to fat intake and consumption of meat and meat products was not very high. For (energy-adjusted) fat intake and meat consumption, this was mainly caused by the relatively small variation in intake in the population. For consumption of meat products, which varied much more in the population studied, the relatively low validity may be attributable to underreporting which may have varied among subjects (Groldbohm et al.,submitted). Taking into account these limitations, there appears nevertheless to be a considerable difference in risk for colon cancer in this population between meat (and fat) consumption on the one hand and meat products on the other, 
the latter showing a consistently increasing risk with increasing consumption in men as well as women. The consumption of (fresh) meat and specific types of meat (beef, pork, minced meat and chicken), in contrast, does not display any trends in risk, whereas the highest quintile is lower than unity most of the time.

We also have to consider the possibility that the results can be explained by confounding by dietary or other determinants of colon cancer. However, we have adjusted for intake of dietary fiber and Quetelet index, which resulted in virtually unchanged relative rate estimates. Other nutrients were no or only weak determinants of colon cancer. Smoking and alcohol consumption have also shown to be hardly related to colon cancer in this data set (Goldbohm et al., submitted for publication).

Comparing our results with findings of others, we may conclude that those for (fresh) meat are in agreement with the substantial number of epidemiologic studies showing no association $(7,9,10,14,21-27)$. The consumption of meat products or processed meat has been investigated in a smaller number of studies $(7,9,12,14,21,22,25-34)$. Most of these studies, however, did not find an increased risk for (types of) processed meat, with exception of Bjelke (18), Young et al. (27) (for lunchmeat only), Willett et al.(12) and Thun et al.(14). This does not necessarily mean that our finding for meat products is a chance finding. Meat products differ from fresh meat in that they have been processed, i.e. cured after the addition of preservatives (salt, nitrite, smoke) and other additives (phosphate, glutamate, ascorbic acid). In the Dutch population, (fresh) meat, usually beef, pork, minced meat or chicken, or fish are part of the hot meal, which is taken once per day and further includes vegetables and (usually) potatoes. Meat products, on the other hand, may or may not constitute part of the sandwich meals, which are taken by most people twice daily. It may be that in this population the circumstances in which meat products are eaten - as sandwich without vegetables and often without fruits in the same meal - are important determinants for the risk. Unfortunately, we do not yet have a sufficient number of cases to explore these possibilities.

The conflicting results between studies regarding meat consumption and colon cancer risk may be attributable to a number of sources. First, the validity of the dietary questionnaire may have been insufficient in some studies. This is in particular critical when the variability in the study population with respect to meat consumption and fat intake is small. Second, the age of study population differed between studies. Available evidence suggests that associations may be stronger at younger ages (7). This may be one of the explanations for the positive result in the Nurses' Health Study, which is based on a relatively young cohort (12). Third, risk of colon cancer may depend on the method of preparation of the meat (products), which is likely to differ between populations. Gerhardsson de Verdier et al.(32) observed an increased risk for subjects who preferred meat with a heavily browned surface. There appears, however, to be no clear relationship between risk and the temperature at which meat is prepared $(27,32,35)$. We did not inform about methods of meat preparation in our study, but in this country it is usuaily panfried or stewed. Last but not least, one of the most plausible explanations is the population level of and variability in the consumption of other foods, such as (specific) vegetables, which may modify the effects of meat consumption $(8,36,37)$. Large studies are required, however, to study effect modification in a relatively homogeneous population.

We conclude from the data presented here that our prospective study does not support the hypothesis that a higher consumption of (fresh) meat increases the risk of colon cancer within the range of meat consumption and fat intake prevailing in the population studied. Consumption of meat products, on the other hand, appears to be associated with an increased risk for colon cancer in this population. These results 
warrant further analysis, in particular in combination with other foods and nutrients, when the number of cases has accrued.

\section{References}

1. Bingham SA. Meat, starch, and non-starch polysaccharides and large-bowel cancer. Am i Clin Nutr 1988; 48: 762-767.

2. Willett W. The search for the causes of breast and colon cancer. Nature $1989 ; 338 ; 389-394$.

3. Kritchevsky D. Meat and cancer. In: Pearson AM. Meat and health. Advances in meat research volume 6. London/New York: Elsevier, 1990: 9-103.

4. Trock B, Lanza E, Greenwald P. Dietary fiber, vegetables, and colon cancer: critical review and meta-analyses of the epidemiologic evidence. J Natl Cancer Inst 1990; 82: 650-661.

5. Phillips KL, Snowdon DA, Brin BN. Cancer in vegetarians. In: Wynder EL et al. (eds). Environmentall aspects of cancer. The role macro and microcomponents and foods. Westport CT: Food and Nutrition Press Inc, 983: 53-73.

6. Bingham SA. Diet and large bowel cancer. J Roy Soc Med 1990; 83: 420-422.

7. Bjelke E. Epidemiology of colorectal cancer, with emphasis on diet. In: Maltoni C. (ed). Advances in tumour prevention, detection and characterization. 1980; Amsterdam: Excerpta Medica, 158-174.

8. Hirayama T. A large scale cohort study on cancer risks by diet - with special reference to the risk. reducing effects of green-yellow vegetable consumption. In: Y. Hayashi et al. (eds), Diet, nutrition and cancer. Tokyo: Japan Sci Soc Press, 1986: 41-53.

9. Phillips RL, Snowdon DA. Dietary relationships with fatal colorectal cancer among Seventh-day adventists. J Natl Cancer Inst 1985; 74: 307-317.

10. Heilbrun LK, Nomura A, Hankin JH, Stemmermann GN. Diet and colorectal cancer with special reference to fiber intake. Int J Cancer 1989; 44: 1-6.

11. Stemmermann GN, Nomura AMY, Heilbrun LK. Dietary fat and the risk of colorectal cancer. Cancer Res 1984; 44: 4633-4637.

12. Willet WC, Stampfer MJ, Coldiz GA, Rosner BA, Speizer FE. Relation of meat, fat, and fiber intake to the risk of colon cancer in a prospective study among women. N Engl J Med 1990; 323: $1664-1672$.

13. Kushi L, Bostick R, McKenzie D, Sellers T, Potter J, Folsom A. Calcium intake and the risk of. colon cancer in a prospective study of 35,216 women (Abstract). Am J Epidemiol (in press).

14. Thun MJ, Calle EE, Namboodiri MM, Flanders WD, Coates RJ, Byers T, Boffetta P, Garfinke! $h_{\text {, }}$ Heath CW. Risk factors of fatal colon cancer in a large prospective study. J Nall Cancer Inst 1992; 84: 1491-1500.

15. Van den Brandt PA, Goldbohm RA, Van 't Veer P, Volovics A, Hermus R.JJ, Sturmans F. A largescale prospective cohort study on diet and cancer in The Netherlands. J Clin Epidemiol 1990; 43: 285-295.

16. Van den Brandt PA, Schouten LJ, Goldbohm RA, Dorant E, Hunen PMH. Development of a record linkage protocol for use in the Dutch cancer registry for epidemiological research. Int J Epidemiol 1990; 19: 553-558.

17. Van den Brandt PA, Van 't Veer P, Goldbohm RA, Dorant E, Volovics A, Hermus RJ, Sturmans F. A prospective cohort study on dietary fat and the risk of postmenopausal breast cancer. Cancer Res (in press)

18. NEVO tabel. Dutch focd composition table 1986-1987. The Hague, Netherlands: Voorlichtingsbureau voor de Voeding, 1986.

19. Willett W, Stampier MJ. Total energy intake: implications for epidemiologic analyses. Am J Epideniol 1986; 124: 17-27.

20. Self SG, Prentice RL. Asymptotic distribution theory and efficiency results for case-cohort studies. Ann Stat 1988; 16: 64-81.

21. Higginson J. Etiological factors in gastrointestinal cancer in man. J Natl Cancer Inst 1966; 37: $527-545$.

22. Graham S, Dayal H, Swanson M, Mittelman A, Wilkinson G. Diet in the epidemiology of cancer of. the colon and rectum. $J$ Natl Cancer Inst 1978; 61: 709-714.

23. Haenszel W, Locke FB, Segi M. A case-control study of large bowel cancer in Japan. I Natl Cancer Inst 1980; 64: 17-22.

24. Kinlen IJ. Meat and fat consumption and cancer mortality: at study of strict religious orders in. Britain. Lancei 1982: 946-949. 
25. Miller AB, Howe GR, Jain M, Craib KJP, Harrison L. Food items and food groups as risk factors in a case-control study of diet and colo-rectal cancer. Int J Cancer 1983; 32: 155-161.

26. Macquart-Moulin G, Riboli E, Cornée J, Charnay B, Berthezène P, Day N. Case-control study on colorectal cancer and diet in Marseilles. Int J Cancer 1986; 38: 183-191.

27. Young TB, Wolf DA. Case-control study of proximal and distal colon cancer and diet in Wisconsin. Int J Cancer 1988; 42: 167-175.

28. Haensel W, Berg JW, Segi M, Kurihara M, Locke FB. Large-bowel cancer in Hawaiian Japanese. J Natl Cancer Inst 1973; 51: 1765-1779.

29. La Vecchia C, Negri E, Decarli A, D'Avanzo B, Gallotti L A case-control study of diet and colorectal cancer in northern Italy. Int J Cancer 1988; 41: 492-498.

30. Tuyns AJ, Kaaks R, Haelterman M. Colorectal cancer and the consumption of foods: a case-control study in Belgium. Nutr Cancer 1988; 11: 189-204.

31. Benito E, Obrabor A, Stiggelbout A, Bosch FX, Mulet M, Muñoz N, Kaldor J. A population-based case-control study of colorectal cancer in Majorca. I. Dietary factors. Int J Cancer 1990; 45: 69-76.

32. Gerhardsson de Verdier M, Hagman U, Peters RK, Steineck G, Overvik E. Meat, cooking methods and colorectal cancer: a case-referent study in Stockholm. Int J Cancer 1991; 49: 520-525.

33. Bidoli E, Franceschi S, Talamini R, Barra S, La Vecchia C. Food consumption and cancer of the colon and rectum in north-eastern Italy. Int J Cancer 1992; 50: 223-229.

34. Peters RK, Pike MC, Garabrant D, Mack TM. Diet and colon cancer in Los Angeles County, California. Cancer Causes Control 1992; 3: 457-473.

35. Lyon JL, Mahoney AW. Fried foods and the risk of colon cancer. Am J Epidemioll 1988 "128: 1000-1006.

36. Manousos O, Day NE, Trichopoulos D, Gerovassilis F, Tzonou A, Polychronopoulou A. Diei and colorectal cancer: a case-control study in Greece. Int J Cancer 1983; 32: 1-5.

37. Lee HP, Gourley L, Duffy SW, Estève J, Lee L, Day NE. Colorectal cancer and diet in an Asian population - a case-control study among Singapore Chinese. Int J Cancer 1989; 43: 1007-1016. 



\title{
Chapter 14
}

\section{Cholecystectomy and colorectal cancer: evidence from a cohort study on diet and cancer*}

\author{
R. Alexandra Goldbohrn ${ }^{1,2}$, Piet A. van den Brandt', Pieter van 't Veer' ${ }^{1}$, \\ Elisabeth Dorant ${ }^{2}$, Ferd Sturmans ${ }^{2}$, Rudolph J.J. Hermus ${ }^{1}$. \\ 1) TNO Toxicology and Nutrition Institute, Zeist, the Netherlands; 2) Universiny of Limburg, Department of \\ Epidemiology, Maastricht, the Netherlands
}

\begin{abstract}
The association between cholecystectomy and subsequent risk for colorectal carclnoma was Investigated In a prospective cohort study on dlet and cancer, whlch is being conducted In the Netherlands from 1986 onwards among 120,852 men and women, aged 55-69. After a follow-up pertod of 3.3 years, 478 Incident cases of colorectal cancer (258 men and 220 women) were Identifled in the cohort, 64 of whom reported at baseline to have undergone previous gallbladder surgery ( 21 men and 43 women). After adjustment for age and famllial history of large bowel cancer, the relative rate (RR) for colorectal cancer In cholecystectomized subjects compared to non-cholecystectomlzed subjects was 1.81 in men $(p=0.02)$ and 1.47 in women $(p=0.05)$. Additional adjustment for parity, Quetelet index, alcohol Intake and other dietary varlables resulted In an RR of 1.78 In men and 1.51 In women. In women, the highest RR was detected In the right colon ( $R R=1.89$ ), whereas in men, no specinc segment of the large bowel accounted specifically for the increased relative rate. In both men and women, the rate appeared to increase from approximately six years after cholecystectomy onward. Accordling to the TNM stage of the disease, patients who had undergone cholecystectomy were not detected at an earller stage than the other patients. It is concluded that in thls study the positive association between colorectal cancer and cholecystectomy cannot be explained by detection bias or ascertainment blas and is not confounded by risk factors for gallstone dlsease or dietary factors.
\end{abstract}




\section{Introduction}

Over the last decade, many studies have been published on the association between cholecystectomy and colorectal cancer. Many of these, often small, studies have reported a positive association. The possibly increased risk for colorectal cancer after removal of the gallbladder is often explained by the higher turnover of bile acids; bacterial degradation then increases the proportion of secondary bile acids (mainly deoxycholic: acid) in the bile and thus in the large intestine (1). It has also been shown that the mitotic index of the colonic mucosa, as indicator of cell proliferation, increases after cholecystectomy (2).

Among the studies with a reasonable number of cases (e.g., more than 30 cancer cases with cholecystectomy), the case-control studies by Vernick and Kuller (3), Moorehead et al. (4), Lee S.S. et al. (5) and Berkel et al. (6) and the follow-up studies by Linos et al. (7) in the USA and Nielsen et al. (8) in Iceland reported a positive association. The last study found the increased risk in men only, as in the autopsy study by Breuer et al. (9) on gallstones and colorectal cancer. Many of the former studies found the highest risk for right-sided colon cancer $(3,4,6,7)$, sometimes restricted to women only. Some groups compared right-sided with left-sided colon cancer cases (3,10-13). All but Abrams et al. (12) found an increased relative risk (RR) for rightsided colon cancer, varying from 1.5 to 2.4. However, a number of large studies reporied no association at all (14-16); even a negative association has been reported from a large follow-up study in Sweden (17). Trying to explain the findings, some $(15,18)$ have suggested that the positive association may be due to an artifact such as "medical attention" or "detection" bias: people seeking medical care for gallstone disease, who are more likely to undergo a cholecystectomy, may also tend to visit a doctor earlier for symptoms of colorectal cancer. Consequently, cancer in patients who underwent cholecystectomy would then be diagnosed at an earlier stage compared to those in other patients and might therefore artifactually increase relative risk

Another explanation may be "ascertainment" bias, which is introduced when ascertainment of previous cholecystectomy is more thorough for cases than for controls. For example, this may happen when hospital records are used to determine whether study subjects have undergone gallbladder surgery: it is conceivable that colorectal cancer patients, during their extensive diagnostic work-up and surgery, are more likely to have cholecystectomy recorded, if they ever had it, than control subjects with other diseases such as breast or lung cancer. However, this explanation may not be very plausible for studies comparing right-sided with left-sided colon cancer. Further explanations for the inconsistent findings include confounding or effect modification by other risk factors for colorectal cancer, such as diet. Most of the published studies controlled for age and sex only, presumably because other data were not available. The very few studies that controlled for dietary factors $(19,20)$ were very sinall.

We have conducted a prospective cohort study among more than 120,000 middleaged men and women with the primary purpose to evaluate the relation between diet and cancer (21). Risk factors for gallstone disease and colorectal cancer as well as the prevalence and age at which cholecystectomy took place were assessed. The availability of this information, together with data on the TNM stage, present for a large part of the colorectal cancers diagnosed, provided the opportunity to assess whether the suggested biases may have had any effect on the association. 


\section{Subjects and methods}

\section{The cohort}

The prospective cohort study on diet and cancer has been initiated in the Netherlands in September 1986. The cohort included 58,279 men and 62,573 women aged 55-69 at the start of the study. The study poptiation originated from 204 municipal population registries throughout the country. A.t baseline, the cohort members, completed a mailed, self-administered questionnaire on dietary habits and other risk factors for cancer. For data processing and analysis the case-cohort approach was used: the cases were enumerated for the entire cohort, while the person years at risk accumulated in the cohort were estimated from a random sample (subcohort). A subcohort of 3500 subjects ( 1688 men, 1812 women) was sampled from the cohort after baseline measurement and was followed up for vital status over 3.3 years. The study design has been described in detail elsewhere (21).

\section{Follow-up for cancer}

Follow-up for incident cancer was established by computerized record linkage with all nine regional cancer registries in the Netherlands and with PALGA, the Dutch national data base of pathology reports. The method of record linkage has been published previously (22). The present analysis is restricted to cancer incidence in the period from September 1986 (baseline measurement) to December 1989, i.e. a followup period of $\mathbf{3 . 3}$ years. In this period, completeness of follow-up (i.e. ratio between number of observed and number of expected cases) of the cohort through linkage with the cancer registries and PALGA together is estimated to be $95 \%$ (23). After excluding subjects who reported a history of cancer other than skin cancer in the baseline questionnaire, a total of 478 incident cases with microscopically confirmed primary colorectal adenocarcinoma were identified (258 men and 220 women). Colorectal cancer was classified according to site as follows. Right colon: cecum through transverse colon (ICD-Oncology codes: 153.0, 153.1, 153.4, 153.5, 153.6); left colon: splenic flexure through sigmoid colon (ICD-Oncology codes: 153.2, 153.3, 153.7); rectum: rectosigmoid and rectum (ICD-Oncology codes: 154.0 and 154.1).

To investigate whether detection bias played a role in the association between cholecystectomy and colorectal cancer, the anatomical extent of the primary tumor (T), as assessed according to the criteria of the TNM classification, was used to compare cancer patients who had undergone cholecystectomy with those who had not. The TNM stage was available for 258 cases, all originating from the cancer registries (from four of the nine registries for cases diagnosed in 1989 only). The PALGA registry, the source for $19 \%$ of all colorectal cases, did not include data on stage of disease.

\section{Questionnaire}

A self-administered questionnaire was used at baseline to collect data on dietary habits, and (possible) risk factors for colon, rectum and other cancers. The dietary part of the questionnaire, concentrating on habitual food intake during the year preceding the start of the study, includes 150 food items and has been validated against a 9-day diet record. Other factors relevant to the association between cholecystectomy and colorectal cancer concern: lifetime history of smoking habits, anthropometry (height and weight), reproductive history (for women only) and familial history of cancer. To study the association between colorectal cancer and the prevalence of (symptomatic) gallstone disease as well as cholecystectomy, the following two questions were included in the questionnaire: (a) "Has a doctor ever diagnosed gallstones, and at what age?" and 
(b) "Did you ever had gallbladder surgery, and at what age?". The applicable age was recorded in five-year age categories.

\section{Data analysis}

Questionnaire data were processed for all 478 incident colorectal cases in the cohort and for all subcohort members. After excluding prevalent cancer cases other than skin cancer from the subcohort, 3346 subjects (1630 men and 1716 women) remained in this group. The questionnaire data were key-entered twice and processed in a manner blinded with respect to case/cohort status in order to minimize observer bias in coding and interpretation of the data. Mean daily nutrient intake was calculated from the dietary questionnaire using the computerized Dutch food composition table (24). Energy adjustment of nutrient intakes was done according to Willett and Stampfer (25). Approximately $7 \%$ of the subjects were excluded for the analyses including dietary variables, because of incomplete or inconsistent dietary data. Quetelet index $\left(\mathrm{kg} / \mathrm{m}^{2}\right)$, was used as measure of obesity. Data were analysed using the case-cohort approach (26), assuming exponentially distributed survival times in the follow-up period. Since standard software was not available for this type of analysis, specific programs were developed to account for the additional variance introduced by sampling from the cohort instead of using the entire cohort (23).

Apart from the known risk factors for gallstone disease (age, Quetelet index, parity and possibly alcohol intake), the following variables were evaluated for confounding: large bowel cancer in first-degree relatives, smoking, level of education, intake of energy and energy-adjusted intake of fat, protein from meat, dietary fiber, vitamin $\mathrm{C}$ and calcium. To evaluate the effect of the time interval between cholecystectomy and diagnosis of colorectal cancer on relative rates, we calculated the interval between age at cholecystectomy, which was taken at the mid-point of the reported five-year age category, and age at start of the cohort study. Age at start of the study was chosen as end-point of the interval (instead of age at diagnosis of cancer) since the follow-up period was short and this end-point was the only strictly comparable end-point available. for both cases and subcohort. The calculated intervals were divided into four categories each containing an approximately equal number of cholecystectomy subjects in the entire data set.

\section{Results}

Table 1 presents the self-reported prevalences of ever diagnosed gallstones and of cholecystectomy categorized by age and sex in the subcohort. Restricting the subcohort to subjects with complete dietary questionnaires did not change these prevalence figures. The prevalence of cholecystectomy in women $(13.3 \%$ ) was approximately three times higher than that in men $(4.7 \%)$. Although the prevalence of reported gallstones was, as expected, higher than that of cholecystectomy, the agreement between the two was very high: $78.5 \%$ of the subjects who reported gallstones ( 72.8 and 80.7 for men and women, respectively), also reported cholecystectomy, while $87.5 \%$ of the cholecystectomized subjects reported to have had gallstones.

Table 2 shows data on the cross-sectional association between cholecystectomy and established risk factors for gallstone disease. In women a non-significant positive association is seen for the Quetelet index. Alcohol intake was negatively associated with cholecystectomy in both men and women. Nulliparous women reported significantly less cholecystectomies than parous women $(8.4 \%$ and $14.5 \%$ respectively; $p<0.01)$, but the prevalence of cholecystectomy did not increase further with parity. Adjustment for age did not change these associations. 
Table 1. Prevalence of gallstones and cholecystectomy (\%) by age at baseline and sex in the complete subcohort $(n=3500)$.

Age category

55-59 60-64 $\quad 65-69 \quad$ Total

\section{Men $(n=1688)$}

Gallstones

Cholecystectomy

Women $(\mathrm{n}=1812)$

Gallstones

Cholecystectomy

\section{5}

2.8

12.4

10.3
6.4.

5.4 .

15.7

15.0
8.0

6.3

5.7

4.7

14.7

13.3

Table 2. Association between prevalence of cholecystectomy and risk factors for gallstone disease in 3346 subcohor menbers free of cancer.

\begin{tabular}{|c|c|c|c|c|c|c|}
\hline & \multicolumn{3}{|c|}{ Men $(n=1630)^{*}$} & \multicolumn{3}{|c|}{ Women $(n=1716)^{*}$} \\
\hline & Not & Yest & $\%$ yes & Not & Yest & $\%$ yes \\
\hline \multicolumn{7}{|c|}{ Ouctelet index $\left(\mathrm{kg} / \mathrm{m}^{2}\right)$} \\
\hline$\leq 22$ & 304. & 17 & 5.3 & 385 & 51 & 11.7 \\
\hline $23 ;-24$ & 480 & $22 !$ & 4.3 & 396 & 60 & 13.2 \\
\hline $25-26$ & $398:$ & 17 & 4.1 & 289 & 44 & 13.2 \\
\hline$\geq 27$ & 310 & 17 & 5.2 & 358 & 69 & 16.2 \\
\hline$p$-value for trend & \multicolumn{3}{|c|}{0.92} & & \multicolumn{2}{|c|}{0.06} \\
\hline \multicolumn{7}{|c|}{ Alcohol consumption (g/day) } \\
\hline no & 230 & 16 & 6.5 & 430 & 83 & 16.2 \\
\hline$\leq 4$ & 309 & 16 & 4.9 & 512 & 70 & 12.0 \\
\hline $5-14$ & 427 & 14 & 4.1 & 247 & 39 & 13.6 \\
\hline $15-29$ & 340 & 18 & 5.0 & 126 & 16 & 11.3 \\
\hline$\geq 30$ & 211 & 10 & 45 & 52 & 4 & 7.1 \\
\hline p-value for trend & \multicolumn{3}{|c|}{0.39} & & \multicolumn{2}{|c|}{0.04} \\
\hline \multicolumn{7}{|l|}{ Parity } \\
\hline 0 & & & & 274 & 25 & 8.4 \\
\hline 1 & & & & 117 & 21 & 15.2 \\
\hline 2 & & & & 320 & 35 & 34.7 \\
\hline$\geq 3$ & & & & 749 & 125 & 14.3 \\
\hline p-value for trend & & & & \multicolumn{3}{|c|}{0.03} \\
\hline
\end{tabular}

- Numbers may not add up to 1630 and 1716 respectively, due to missing values for some of the variables.

+ No: no cholecystectomy reported; yes: cholecystectomy reported.

Table 3 shows the results of the prospective cohort analysis, i.e. the relative rates (RR) for colorectal cancer by cholecystectomy status, adjusted for risk factors for 
gallstone disease from Table 2 as well as for other dietary confounders. Both men and women with cholecystectomy had an increased rate for colorectal cancer $(\mathbf{R} R=1.81$, $\mathrm{p}=0.023$ and $\mathrm{RR}=1.47, \mathrm{p}=0.052$, respectively). Adjustment for the confounding variables affected estimated relative rates only slightly. For women, adjustment for confounding by parity, Quetelet index and alcohol intake increased the estimated relative rate to 1.55 (data not shown). The slight increase was virtually cancelled out by further adjustment for dietary variables ( $R R=1.51$ ). Inclusion in the multivariate models of smoking, level of education and intake of vitamin $\mathrm{C}$ and calcium did not change the estimates. In women, the relative rate after cholecystectomy appeared to be highest for the right colon ( $\mathrm{RR}=1.89$ ); other sites showed less and non-significantly increased relative rates. In men, no specific subsite accounted for the increased risk.

Table 3. Adjusted relative rate of colorectal cancer in cholecystectomized subjects compared to noncholecystectomized subjects, according to sex and subsite.

\section{Number of}

of cases

$\mathrm{RR}+\quad 95 \% \mathrm{Cl}$

RR

$95 \% \mathrm{CI}$

Men

\begin{tabular}{|c|c|c|c|c|c|}
\hline All cases & $231(19)^{*}$ & 1.81 & $1.06-3.08$ & 1.78 & $1.03-3.08$ \\
\hline Right colon & $61(5)$ & 1.74 & $0.70-4.32$ & 1.66 & $0.61-4.52$ \\
\hline Lefl colon & $68(6)$ & 1.96 & $0.89-4.45$ & 2.22 & $0.90-5.46$ \\
\hline Rectum & $88(7)$ & 1.80 & $0.80-4.05$ & 1.70 & $0.73-3.94$ \\
\hline \multicolumn{6}{|l|}{ Vomen } \\
\hline All cases & $177(34)$ & 1.47 & $0.99-2.19$ & 1.51 & $1.02-2.23$ \\
\hline Rigltht colon & $62(14)$ & 1.69 & $0.93-3.08$ & 1.89 & $1.04-3.42$ \\
\hline Lelt colon & $51(9)$ & 1.33 & $0.63-2.81$ & 1.25 & $0.60-2.59$ \\
\hline Rectum & $48(10)$ & 1.62 & $0.81-3.24$ & 1.55 & $0.73-3.27$ \\
\hline
\end{tabular}

- In parenthesis: number of cises with cholecysiectomy. Number of cases less than total number in the study due to missing values for several variables used for adjustment. In 30 cases (14 men and 16 women), virtually all originating from the PALGA registry, subsite is unknown.

† Reference category: no cholecystectomy. Adjusted for age (year) and large-bowel cancer in first-degree relatives.

\$ Reference category: no cholecystectomy. Adjusted for age (year), large-bowel cancer in first-degree relatives, Quetelet index, parity (women only), intake of energy, alcohol and energy-adjusted intakes of fat (men only), meat protein (men only) and dietary fiber.

Table 4 shows the relative rates of colorectal cancer classified by interval between cholecystectomy and start of the study. In both men and women, a significant trend with increasing length of the interval was detected; the increased rates appeared to be limited to the intervals greater than six years.

Table 5 presents data on the pathological stage of the primary tumor in the cancer cases according to cholecystectomy status. Neither men nor women with cholecystectomy showed a trend towards earlier stage of disease at diagnosis. In women an inverse trend could be detected. 
Table 4. Relative rate of colorectal cancer classified by time interval between cholecystectomy and the start of the study.

\begin{tabular}{|c|c|c|c|c|c|c|}
\hline \multirow[t]{2}{*}{ Time interval } & \multicolumn{2}{|c|}{ Number of cases ${ }^{*}$} & \multirow{2}{*}{$\frac{\text { Men }}{\text { RR† }}$} & \multirow{2}{*}{$\frac{\text { Women }}{\text { RRt }}$} & \multicolumn{2}{|c|}{ Both sexes } \\
\hline & Men & Women & & & $\mathbf{R R} \ddagger$ & $95 \% \mathrm{Ci}$ \\
\hline No cholecystectomy & 212 & 143 & 1.00 & 1.00 & 1.00 & \\
\hline 0. 6 years & 4 & 5 & 1.12 & 1.19 & 1.19 & $0.60-2.36$ \\
\hline $7-13$ years & 6 & 8 & 1.99 & 1.82 & 1.83 & $1.02-3.29$ \\
\hline $14-23$ years & 7 & 9 & 3.66 & 1.56 & 1.91 & $1.08-3.36$ \\
\hline$\geq 24$ years & 2 & 11 & 1.12 & 1.66 & 1.66 & $0.91-3.02$ \\
\hline p-trend & & & 0.044 & 0.030 & 0.001 & \\
\hline
\end{tabular}

- For 1 female colorectal cancer case age at cholecystectomy was unknown.

t Adjusted for age (year), large bowel cancer in first-degree relatives, Quetelet index, parity (women only), intake of energy , alcohol and energy-adjusted intakes of fat (men only), meat protein (men only) and dietary fiber.

\# The pooled estimate for both sexes was adjusted for sex, age, large bowel cancer in first-degree relatives, Quetelet index and alcohol intake.

Table 5. Number of incident cases of colorectal cancer classified by cholecystectomy status and sex, and according to the final pathology stage (TNM) of the disease.

\begin{tabular}{|c|c|c|c|c|c|c|c|c|}
\hline \multirow[t]{3}{*}{ TNM stage } & \multicolumn{4}{|c|}{ Men } & \multicolumn{4}{|c|}{ Women } \\
\hline & \multicolumn{2}{|c|}{ No* } & \multicolumn{2}{|c|}{ Yes* } & \multicolumn{2}{|c|}{ No ${ }^{*}$} & \multicolumn{2}{|c|}{ Yes* } \\
\hline & n & $\%$ & n & $\%$ & $\mathrm{n}$ & $\%$ & n & $\%$ \\
\hline Total number of cases & 235 & & 21 & & 177 & & 43 & \\
\hline TNM available & 122 & 100.0 & 11 & 100.0 & 103 & 100.0 & 28 & 100.0 \\
\hline$T 1+T 2$ & 54 & 44.3 & 5 & 45.5 & 4.1 & 39.8 & 7 & 25.0 \\
\hline $13+T 4$ & 64 & 52.5 & 6 & 54.5 & 60 & 58.3 & 21 & 75.0 \\
\hline $\mathrm{TX}+$ & 4 & 33 & 0 & 0 & 2 & 1.9 & 0 & 0 \\
\hline
\end{tabular}

- No: no cholecystectomy reported; yes: cholecystectomy reported.

$\dagger$ Stage of primary tumor not assessable.

\section{Discussion}

Our findings are supportive of a positive association between cholecystectomy and colorectal cancer. Considering the prospective nature of our study - all participants recorded gallbladder surgery irrespective of the later occurrence of cancer - , it is unlikely that ascertainment bias explains these findings. It is also unlikely that detection bias plays a major role, since we have shown that cholecystectomized colorectal cancer patients were diagnosed with equally advanced cancers as patients who did not have 
previous cholecystectomy; in women even an inverse trend could be observed. Although the data on TNM classification were not available for all cases, there is no reason to expect different findings in a complete data set, since the availability of TNM depended on source and year of registration and is thus likely to have influenced the results at random.

A criticism on the study might be the method used to determine presence of cholecystectomy by self-reporting without any check. The data themselves, however, appear to refute this. First, the prevalence of cholecystectomy measured in the subcohort agreed very well with that found in a recent survey in the south of the Netherlands, in which subjects were interviewed and cholecystectomy diagnoses were verified by either hospital records or ultrasound examination (27). The prevalences for men in that survey $(n=182)$ were 2.5 and $5.8 \%$ in the age groups 50 to 59 and 60 to 69 respectively. For women $(n=215)$ the corresponding figures were 13.1 and $14.1 \%$. Cholecystectomy rates are very similar throughout the country, as was shown by autopsy studies in six cities (28). Furthermore, in the subcohort restricted to subjects with complete dietary questionnaires the same prevalences were found, indicating that subjects with incomplete (dietary) questionnaires, who also might have skipped the question on galibladder surgery, did not underreport cholecystectomy. Finally, established risk factors for gallstone disease investigated in this study, i.e. age, sex, parity (29) and Quetelet index (30), were all found to be associated with cholecystectomy in the anticipated direction. Also alcohol consumption, which has often been shown to be inversely related to gallstone disease (30), was negatively associated with cholecystectomy in our data although, of course, this may have been a consequence rather than a cause of gallstones and thus cholecystectomy. We therefore conclude that underreporting of previous cholecystectomy is not likely to have been substantial. There might have been subjects who falsely reported gallbladder surgery. Although in self-reporting this is probably less likely to happen than false-negative reporting, under- and overreporting acting together will lead to misclassification that is presumably non-differential with respect to prospectively recorded disease status. In that case we would have observed a lower relative risk than the true one (31). Underreporting alone does not change the estimated relative risk.

Adjustment for confounding by risk factors for gallstone disease and by dietary factors did not strongly influence the estimated relative rates. It is unlikely, therefore, that in this study the positive association between cholecystectomy and colorectal cancer has been confounded by any of the factors investigated. This also suggests that gallstone disease is not the (sole) underlying causal link between cholecystectomy and colorectal cancer, as proposed by some (32). Unfortunately, no prospective study so far (including ours) comprised enough subjects with unoperated gallstone disease to evaluate this directly. Moreover, parity and obesity, important risk factors for gallstones in women, were found to be slightly inversely associated with colorectal cancer. Although we cannot exclude that other factors could explain the positive association (e.g. physical activity), the investigated more obvious factors do not appear to be important in this respect. This conclusion does not imply that the (lack of an) association reported in other studies is not influenced by confounding, because this depends on the distribution of potential confounders in a population. Non-adjustment for parity is likely to have underestimated the relative risk for women in other studies, since parity is a risk factor for gallstone disease, but has been found protective against colorectal cancer in a number of populations (33) including our population. However, the degree of underestimation of the relative risk by not adjusting for parity is difficult to assess and is likely to differ from one study to another. The role of parity in the relation between cholecystectomy and colorectal cancer also suggests that cholecystectomy rather than 
shared risk factors for gallstone disease and colorectal cancer causes (at least part of) the increased risk.

If the association between cholecystectomy and colorectal cancer were causal, one would expect the relative risk to start increasing some years after removal of the gallbladder. This is precisely what is suggested by our data on the time interval between age at cholecystectomy and age at the start of the study, although the number of cases in each interval category is too small to draw definite conclusions about the strength of the associations in separate intervals. Neither is it possible to determine exactly the length of the interval after which the relative rate starts to depart from unity, since the starting point of each individual interval was defined as the mid-point of a five-year age category and the end-point preceded the diagnosis of cancer by three years at most. This implies that the true individual interval was within the range of the calculated interval minus two and plus five years. Few other studies have presented data on the association within interval categories. Four of seven studies show a relative risk close to one in the first years after cholecystectomy $(3,5,8,15)$. Even the large study by Friedman et al.(15), which did not find an overall relative risk different from unity, shows evidence for an increased relative risk from five years after cholecystectomy onward. Spitz et al. (12), who used left-sided colon cancer cases as controls for right-sided colon cancer cases, did not observe a risk gradient with time interval. Kune et al. (16) and Berkel et al. (6) reported a highly increased risk in the first few years directly following cholecystectomy, which may be attributed to confounding of symptoms of (right-sided) colon cancer with those of gallstone disease (16).

In men, we found an increased relative rate for all subsites; in women, the relative rate appeared to be highest for the right side of the colon. This result in women is in accordance with many other studies $(3-7,10,16,34)$. We require, however, more cases to draw more definite conclusions with respect to subsite.

In conclusion, our study corroborates the evidence for a positive relationship between cholecystectomy and colorectal cancer, unlikely to be attributable to many well-known methodological biases. The data also provide support for the possibility that cholecystectomy is the causal factor rather than (predisposition for) gallstone disease. An intact gallbladder empties only after a (fat-containing) meal, whereas after cholecystectomy bile from the bile duct trickles continuously into the gut. The increased risk after cholecystectomy may be attributed to the difference in composition and content between bile from the gallbladder and that from the bile duct, but also to the increased time that the gut is exposed to bile. The latter possibility is consistent with observations that meal frequency and thus a more frequent exposure of the gut to bile also increases risk for colorectal cancer (35-37).

The results warrant further study into differences between subsites and into potential modification of the effect by dietary factors when, after more years of followup, a sufficient number of cases will have been accrued.

\section{References}

1. Moorchead RJ, McKelvey STD. Cholecystectomy and colorectal cancer. Br J Surg 1989; 76:250-253.

2. Bandettini $\bar{L}$, Filipponi $F$, Romagnoli $\bar{P}$. Increase of the mitotic index of colonic mucosa after cholecystectomy. Cancer 1986; 58: 685-687.

3. Vernick IJ, Kuller LH. Cholecystectomy and right-sided colon cancer: an epidemiological study. Lancet 1981: $2: 381-383$.

4. Moorehead RJ, Kernohan RM, Patterson CC, McKelvey STD, Parks TG. Does cholecystectomy predispose to colorectal cancer? A case control study. Dis Colon Rectum 1986; 29: 36-38.

5. Lee SS, Cha S, Lee RL. The relationship between cholecystectomy and colon cancer: An Iowa study. J Surg. Oncol 1989; 41: 81-85. 
6. Berkel J, Hombergen. DAMA, Hooymayers IE, Faber JAJ. Cholecystectomy and colon cancer. Am J Gastroenterol 1990; 85: 61-64.

7. Linos DA, Beard CM, O'Fallon WM, Dockerty MB, Beart Jr. RW, Kurland LT. Cholecystectomy and carcinoma of the colon. Lancet. 1981; 2: 379-381.

8. Nielsen GP, Theodors. A, Tulinius II, Sigvaldason H. Cholecystectomy and colorectal carcinoma: a total-population historical prospective study. Am J Gastroenterol 1991; 86: 1486-1490.

9. Breuer NF, Katschinski B, Mörtl E, Leder LD, Goedbell H Large bowel cancer risk in cholelithiasis and after cholecystectomy. Digestion 1988; 40: 219-226.

10. Peters H, Keimes AM. Die Cholezystektomie, als prädisponierender Faktor in de Genese des Kolorektalen Karzonoms? Deutsche Med. Wochenschr. 1979; 104: 1581.1583.

11. Vernick IJ, Kuller LH, Lohsoonthorn P, Rycheck RR, Redmond CK. Relationship between cholecystectomy and ascending colon cancer. Cancer 1980; 45: 392-395.

12. Abrams JS, Anton JR, Dreyfuss, DC. The absence of a relationship between cholecystectomy and the subsequent occurrence of cancer of the proximal colon. Dis Colon Rectum 1983; 26: 141-144.

13. Spitz MR, Russell NC, Guince VF, Newell GR. Questionable relationship between cholecystectomy and colon cancer. J Surg, Oncol 1985; 30: 6-9.

14. Eriksson SG, Lindström CG. Lack of relationship between cholecystectomy and colorectal cancer: A case control autopsy study in a defined population. Scand J Gastroenterol 1984; 19: 977-982

15. Friedman GD, Goldhaber MK, Quesenberry Jr. CP. Cholecystectomy and large bowel cancer. Lancet 1987; 1: 906-908.

16. Kune GA, Kune S, Watson LF. Large bowel cancer after cholecystectomy. Am J Surg 1988; 156: 359-362.

17. Adami HO, Krusemo UB, Meirik O. Unaltered risk of colorectal cancer within 14-17 years of cholecystectomy: updating of a population-based cohort Study, Br J Surg 1987; 74: 676-678.

18. Barker WH. Cholecystectomy and colon cancer. Lancet 1981; 2: 986.

19. Wu Ẩ, Paganini-Hill A, Ross RK, Henderson BE. Alcohol, physical activity and other risk factors for colorectal cancer: A prospective study. Br J Cancer 1987; 55: 687-694.

20. Lee HP, Gourley L, Duffy SW, Estève J, Lee J, Day NE. Colorectal cancer and diet in an Asian population - A case-control study among Singapore Chinese. Int J Cancer 1989; 43: 1007-1016.

21. Van den Brandt PA, Goldbohm RA, Van 't Veer P, Volovics A, Hermus RJJ, Sturmans F. A largescale prospective cohort study on diet and cancer in The Netherlands. J Clin Epidemiol 1990; 43: 285-295.

22. Van den Brandt PA, Schouten IJ, Goldbohm RA, Dorant E, Hunen PMH. Development of a record linkage protocol for use in the Dutch eancer registry for epidemiological research. Int J Epidemiol 1990; 19: 553.558.

23. Van den Brandt PA, Van 'i Veer P, Goldbohm RA, Dorant E, Volovics A, Hermus RJJ, Sturanans F. A prospective cohort study on dictary fat and the risk of post menopausal breast cancer. Cancer Res (in press).

24. Nivo tabel. Dutch food composition table 1986-1987. The Hague, Netherlands: Voorlichtingsbureau voor de Voeding, 1986.

25. Willeti W, Stampier MJ. Total energy intake: implications for epidemiologie analyses. Am J Epidemiol 1986: 124: 17-27.

26. Seli SG, Prentice RL. Asymptotic distribution theory and efficiency results for case-cohort studies. Ann Stat 1988; 16: 64-68.

27. Thijs $\bar{C}$ Knipschild $P$, Van Engelshoven $J$. The prevalence of gallstone disease in a Dutch population. Scand J Gastroenterol 1990; 25: 155-160.

29. Thijs C, Knipschild P, Leffers P. Pregnancy and gallstone disease = an empiric demonstration of the imporiance of specification of risk periods. Am J Epidemiol 1991; 134: 186-j95.

28. Thijs CT. How common are gallstones? In: Schumpelick V, Winkeltau G. Treutner K-H (eds), 9th Grenzland Symposium on Biliary Surgery Aachen 1990. Stuttgart. Georg Thieme Verlag, 1991.

30. Maclure KM, Hayes KC, Colditz GA, Stampfer MJ, Speizer FE, Willett WC. Weight, diet, and the risk of symptomatic gallstones in middle-age women. N Eng! J Med 1989; 21: 563.569.

31. Rothman KJ. Modern epidemiology. Eloston. Little, Brown and Company, 1986.

32. Narisawa T, Yamazaki $\mathbf{Y}$, Kusaka H, Takahashi $\mathbf{M}$, Kotanagi $\mathbf{H}$, Koyama $\mathbf{K}$. Clinical observation on the association of gallstones and colorectal cancer. Cancer 1991; 67: 1696-1700.

33. Peters RK, Pike MC, Chang WWI, Mack TM. Reproductive factors and colon cancer. Br I Cancer 1990; $61: 741-748$.

34. Mamianetti A, Cinto R.O, Altolaguirre D, Bosicio OA, Heidenreich A, Salomón M. Relative risk of colorectal cancer after cholecystectomy: A multicentre case-control study. Int J Colorect Dis 1988; 3: $215-218$ 
35. Potter JD, McMichael AJ. Diet and cancer of the colon and rectum: a case-control study. J Nat Cancer Inst 1986; 76: 557-569.

36. Deverdier MG, Longnecker MP. Eating frequency - A neglected risk factor for colon cancer. Cancer Causes \& Control 1992; 3: 77-81.

37. Franceschi S, La Vecchia C, Bidoli E, Negri E, Talamini R. Meal frequency and risk of colorectal cancer. Cancer Res 1992; 52: 3589-3592. 



\section{Chapter 15}

\section{A prospective cohort study on selenium status and the risk of lung cancer*}

Piet A. van den Brandt', R. Alexandra Goldbohm ${ }^{1,2}$, Pieter van 't Veer', Peter Bode ${ }^{3}$, Elisabeth Dorant ${ }^{1}$, Rudolph J.J. Hermus ${ }^{2}$, Ferd Sturmans ${ }^{1}$.

1) Department of Epidemiology, Universily of Limburg, Maastricht, the Netherlands; 2) Department of Nutrition, TNO-Toxicology and Nutrition Institute, Zeist, the Netherlands; 3) Interfaculty Reactor Institute, Delft Universiry of Technology, Delft, the Netherlands

\section{Abstract}

Selenium may be anticarcinogenlc because of Its role in the cellular defense system against oxldative stress. The assoclation between toenall selenium (a marker of longterm selenlum status) and lung cancer was investigated In a cohort study on dlet and cancer, that started in 1986 among 120,852 Dutch men and women aged 55-69 years. After 3.3 years of follow-up, 550 microscopically confirmed Incident cases with lung carcinoma were detected. Toenail selenlum data were avallable for 370 lung cancer cases and $\mathbf{2 4 5 9}$ members of a randomly selected subcohort. After controlling for age, gender, smoking and education, the relative rate of lung cancer for subjects in the highest compared to the lowest quintile of toenail selenium was 0.50 ( $95 \%$ confidence interval $0.30-0.81$, with a significant inverse trend across quintlles $(p=0.006)$. The protective effect of selenium was concentrated in subjects with a relatively low dietary intake of beta-carotene or vitamin $C$. The relatlve rate in the highest compared to the lowest guintile of selenium was 0.45 in the low beta-carotene group (95\% confidence interval 0.22-0.92; trend $-\mathrm{p}=\mathbf{0 . 0 2 8}$ ) and 0.36 In the low vitamin $\mathrm{C}$ group (95\% confldence interval $0.17-0.75$; trend-p<0.001). This study supports an Inverse assoclation between selenium status and lung cancer and suggests a modification of the effect of selenium by the antloxidants beta-carotene and vitamin $\mathrm{C}$. 


\section{Introduction}

It has been suggested that selenium has anticarcinogenic potential through its role as component of glutathione peroxidase, an enzyme that is part of the cellular defense system against oxidative damage (1). Animal studies have indicated that selenium compounds may have an inhibitory effect on carcinogenesis in various experimental models, although not all of these studies show a reduced tumour incidence (2). In humans, ecological studies have shown an inverse association between cancer mortality rates and selenium concentrations in forage crops and serum (2,3). Among other tumours, this inverse relationship was found for lung cancer. Since smoking induces oxidative stress $(4,5)$, studying a smoking-related cancer such as lung cancer provides an opportunity to investigate the postulated anticarcinogenic effect of selenium.

Analytic epidemiological studies on selenium and cancer frequently use biologica! markers of selenium status such as serum or toenail selenium levels, because estimation. of dietary selenium intake is considered unreliable (6). Although there are a number of case-control studies that have shown that cases have lower serum selenium levels than control subjects (2), these findings are difficult to interpret because serum levels might be reduced due to a lower intake or sequestration of selenium by tumour cells $(7,8)$. Prospective studies, in which this problem can be avoided, have yielded varying results regarding serum selenium levels and cancer risk (8). Due to their limited size, the majority of these prospective studies was confined to analysis of all tumour sites combined. A potential limitation of the serum studies is the large intraindividual variation in serum selenium levels (6). There is evidence that toenail selenium levels represent the selenium status over several months $(9,10)$ and that the levels reflect differences in selenium intake $(11,12)$. Toenail clippings have been used recently as a long-term biomarker of selenium status in various prospective studies $(13,14)$, but none of these studies dealt with lung cancer. The purpose of our study was to investigate the relationship between prediagnostic toenail selenium levels and lung cancer risk in a large-scale prospective cohort study in the Netherlands. Apart from the overall association, we evaluated associations for specific types of lung carcinoma, considering the evidence that etiological factors may differ between types $(15,16)$. In view of the suggested possible gender-specific effect of selenium (17), analyses were also conducted for men and women separately. The potential effect modification by the antioxidant vitamins beta-carotene and vitamin $C$, as well as retinol was also investigated.

\section{Materials and methods}

The cohort characteristics and the method of cancer follow-up have been described before $(18,19)$. Briefly, the cohort study started in 1986 when 58,279 Dutch men and 62,573 women aged 55-69 years were enrolled in the cohort. At baseline, cohort members completed a self-administered questionnaire on usual dietary intake and potential confounders and also provided toenail clippings. Following the case-cohort approach for analysis of the data, a subcohort of 3,500 subjects (1688 men, 1812 women) was randomly sampled from the cohort after the baseline exposure measurement. The subcohort has been followed up biennially for vital status information in order to estimate the accumulated persontime in the cohort. Incident cancer cases occurring in the cohort have been identified by record linkage to cancer registries and a pathology register. The analysis in this report is restricted to the cancer incidence in the recently completed 3.3 year follow-up period from September 1986 to December 1989. The completeness of cancer follow-up was estimated to be $95 \%$ (20). In these 3.3 years of follow-up, a total of 617 lung cancer cases were detected (542 
men, 75 women). After excluding cases who reported a history of cancer other than skin cancer in the baseline questionnaire $(n=53)$, cases with in situ carcinoma $(n=1)$ and cases without a microscopically confirmed diagnosis $(\mathrm{n}=11), 552$ incident cases remained available for analysis. Because we were interested in the association of toenail selenium with the various types of lung carcinoma, cases with a sarcoma $(n=1)$ or unspecified morphology $(\mathrm{n}=1)$ were also excluded, leaving 550 cases with lung carcinoma ( $488 \mathrm{men}, 62$ women). After excluding prevalent cancer cases other than skin cancer from the subcohort of 3500 as well, 3346 subjects (1630 men, 1716 women) remained in this group. Toenail clippings had been provided by 384 lung cancer cases and 2569 subcohort members. Problems with the detection of toenail selenium (interference by other elements such as calcium) occurred in 1 and 16 of these 384 and 2569 specimens, respectively. In addition, 13 and 94 specimens were excluded from the case and subcohort groups, respectively, because the specimens weighed less than 10 $\mathrm{mg}$, which would yield unreliable selenium measurements. Thus, toenail selenium data on 370 lung cancer cases (335 men, 35 women), and 2459 subcohort members (1211 men, 1248 women) were available for analysis.

\section{Determination of toenail selenium levels}

The toenail selenium analyses were carried out by the Interfaculty Reactor Institute (IRI) at Delft University, the Netherlands. Each analytical batch contained toenail specimens of cases and subcohort members, and specimens were analyzed in a manner blinded with respect to case/subcohort status. Toenails were first cleared by scratching off any debris with a quartz knife. After ultrasonic cleaning with acetone for 15 minutes, distilled water for 10 minutes and acetone for 15 minutes, respectively, the specimens were freeze-dried during 15 hours to eliminate any humidity variations between runs. The selenium content of the toenails was measured by instrumental neutron activation analysis of the metastable-selenium-77 isotope. The specinens were irradiated for 17 seconds in a thermal flux of $1.2 \times 10^{13}$ neutrons. $\mathrm{s}^{-1} \cdot \mathrm{cm}^{-2}$. After a decay time of 20 seconds, gamma radiation of ${ }^{77} \mathrm{~m}$ Se was measured for 60 seconds. The accuracy of the method was checked by analysis of a certified Bovine liver standard (Standard Reference Material 1577a of the US National Bureau of Standards). For 26 determinations, a mean value ( \pm SD) of $0.70 \pm 0.04 \mu \mathrm{g} / \mathrm{g}$ selenium was observed against a certified value of $0.71 \pm 0.04 \mathrm{\mu g} / \mathrm{g}$. The precision of the method was evaluated by duplicate selenium measurements of specimens from 27 randomly selected subjects; the coefficient of variation was 6.6 percent.

\section{Data analysis}

The distribution of various potential confounders known to be associated with lung cancer (gender, age, smoking habits and level of education) in the case and subcohort group were compared as well as the mean toenail selenium levels. The highest attained level of education was classified as follows: primary school only; lower level vocational education (in addition to primary school); secondary school or medium level vocational education; university or higher level vocational education. After categorization of the cases according to histological type of lung carcinoma (squamous cell carciñoma, small cell carcinoma, adenocarcinoma, large cell and other types of carcinoma) or according to the year of follow-up in which the diagnosis was made, the mean toenail selenium concentrations of the case groups were also compared with each other.

Next, case-cohort analyses (21) were conducted, based on the assumption that survival times were exponentially distributed in this follow-up period. For these analyses, toenail selenium levels were categorized into quartiles, or quintiles (depending on the available number of cases) according to the distribution in the subcohort. In the 
gender-age-stratified analyses, we computed Mantel-Haenszel relative rates of lung cancer for each of the quantiles, $95 \%$ confidence intervals, and tests for trend in the relative rates (which were corrected for the additional variance introduced by the subcohort sampling). In the multivariate case-cohort analyses, relative rates (with corrected $95 \%$ confidence intervals) of lung cancer were computed after adjustment for the effects of gender, age, smoking (expressed as packyears for past and current smokers) and highest level of education. The age and smoking variables were entered as continuous variables. Tests for trend were computed based on likelihood ratio tests with scores of 1-5 assigned to increasing quintiles of toenail selenium, as in the stratified analysis. Apart from analyses for the total group, analyses were also conducted for men and women separately and for each histological type of lung carcinoma. The interaction between toenail selenium and smoking was tested using likelihood ratio tests. To evaluate the potential influence of preclinical disease on toenail selenium levels, analyses were also conducted after excluding cases occurring, in the first year of followup. Finally, we analyzed the relationship between toenail selenium and lung cancer with regard to the dietary intake of retinol, beta-carotene and vitamin $C$ (in the subset of people with complete dietary data), to study the potential effect modification by these vitamins. Two-sided p-values are used throughout this report.

\section{Results}

Table 1 provides information on the distribution of gender, age, smoking habits and highest attained level of education among lung cancer cases and subcohort members for whom the toenail selenium level was known. As expected, large differences in the relative frequencies of gender, age and smoking habits were observed between the case and subcohort groups, while smaller differences were found with respect to highest attained level of education. For both men and women, the average toenail selenium concentration was lower among lung cancer cases than among the subcohort members. For men, the mean ( \pm SD) toenail selenium levels in cases and subcohort were 0.529 $( \pm 0.206) \mu \mathrm{g} / \mathrm{g}$ and $0.547( \pm 0.126) \mu \mathrm{g} / \mathrm{g}$, respectively, while for women values of 0.537 $( \pm 0.080)$ and $0.575( \pm 0.109) \mu \mathrm{g} / \mathrm{g}$, respectively, were observed.

When toenail selenium levels of cases according to histological subtype were compared, cases with adenocarcinoma showed the lowest toenail selenium levels while the highest levels were observed among cases with squamous cell carcinoma (table 2). For all four types of carcinoma, male cases showed somewhat lower values than female cases. When cases were categorized with respect to the year of follow-up in which they were diagnosed, there was no trend towards lower toenail selenium levels in cases occurring early in both men and women, indicating no effect of preclinical disease on toenail selenium levels (table 2).

The results of the stratified analysis of toenail selenium and lung cancer risk are shown in table 3 . Toenail selenium was inversely associated with the risk of lung cancer in this analysis (test for trend $p<0.001$ ). The relative rate of lung cancer in the highest quintile of toenail selenium compared to the lowest quintile was 0.40 (95\% confidence interval (CI) 0.27-0.59).

In the multivariate analyses we additionally adjusted for smoking and level of education; the additional information on packyears of smoking and level of education was available for 317 lung cancer cases and 2311 subcohort members. The results, shown in table 4 , indicate an inverse association between toenail selenium and lung eancer risk among the total group of subjects (trend-p=0.006), with a relative rate of lung cancer for those in the highest quintile compared to the lowest quintile of toenail selenium of $0.50(95 \% \mathrm{CI} 0.30-0.81)$. 
Table 1. Distribution of lung cancer cases and subcohort members with complete toenail selenium data according to various characteristics.

\section{Characteristic}

\section{Cases}

n* $\quad(\%)$

370

Total

Gender

Men

Women

Age (years)

$55-59$
$60-64$
$65-69$

Smoking habits

Never smoked

Ex-smoker

Current smoker

Highest level of education

Primary school

Lower vocational education

Secondary school/medium vocational

University/higher vocational

6
109

(29.6)

$\begin{array}{rrr}335 & (90.5) & 1211 \\ 35 & (9.5) & 1248\end{array}$

(49.2)

$(50.8)$

$\begin{array}{llll}100 & (27.0) & 941 & (38.3) \\ 134 & (36.2) & 855 & (34.8) \\ 136 & (36.8) & 663 & (26.9)\end{array}$

( 1.6$)$

(68.8)

863

852

(35.2)

(34.7)

740

(30.1)

$\begin{array}{rrrr}117 & (32.3) & 745 & (30.7) \\ 102 & (28.2) & 542 & (22.3) \\ 108 & (29.8) & 833 & (34.3) \\ 35 & (9.7) & 310 & (12.8)\end{array}$

Toenail selenium $(\mu \mathrm{g} / \mathrm{g})$
Men (mean $\pm \mathrm{SD}$ )
Women (mean \pm SD)
$0.529 \pm 0.206$
$0.547 \pm 0.126$
$0.537 \pm 0.080 \quad 0.575 \pm 0.109$

- Due to missing questionnaire data, numbers may not add up to 370 and 2459 , respectively.

Table 2. Toenail selenium levels $(\mu \mathrm{g} / \mathrm{g}$ ) in male and female lung cancer cases according to histological subtype and year of follow-up.

Group

Men

Women

Group

n Mean $\pm S D \quad$ n Mean $\pm S D$

Histological subtype

Squamous cell carc.

Small cell carc.

Adenocarcinoma

Large cell, other carc.

$\begin{array}{rl}161 & 0.541 \pm 0.278 \\ 56 & 0.520 \pm 0.099 \\ 65 & 0.518 \pm 0.100 \\ 53 & 0.517 \pm 0.113\end{array}$

$\begin{array}{rr}9 & 0.547 \pm 0.101 \\ 8 & 0.531 \pm 0.079 \\ 10 & 0.527 \pm 0.063 \\ 8 & 0.544 \pm 0.089\end{array}$

Year of follow-up

1
2
3
4

$\begin{array}{rr}93 & 0.540 \pm 0.348 \\ 104 & 0.530 \pm 0.123 \\ 113 & 0.516 \pm 0.092 \\ 25 & 0.548 \pm 0.146\end{array}$

$7 \quad 0.572 \pm 0.045$

$13 \quad 0.530 \pm 0.089$

$11 \quad 0.551 \pm 0.079$

$25 \quad 0.548 \pm 0.146$

$4 \quad 0.458 \pm 0.066$ 
Table 3. Mantel-Haenszel relative rate of lung cancer according to toenail selenium level in gender-agestratified analysis.

Toenail selenium level (quintile boundaries in $\mu \mathrm{g} / \mathrm{g}$ ).

\section{No. of}

cases
Person years

in subcohort
$\mathrm{RR}_{\mathrm{MH}}$

(95\% CI)

\begin{tabular}{|c|c|c|c|c|}
\hline$(\leq 0.483)$ & 134 & 1598 & $1.00^{*}$ & \\
\hline$(\leq 0.530)$ & 75 & 1597 & 0.64 & $(0.47-0.89)$ \\
\hline$(\leq 0.573)$ & 69 & 1593 & 0.66 & $(0.47-0.92)$ \\
\hline$(\leq 0.630)$ & 53 & 1587 & 0.56 & $(0.39-0.80)$ \\
\hline$(>0.630)$ & 39 & 1609 & 0.40 & $(0.27-0.59)$ \\
\hline est for trend & & & 23.159 & $(<0.001)$ \\
\hline
\end{tabular}

* Reference category.

Because of the high proportion of male lung cancer cases, the association with toenail selenium among men strongly resembles that in the total group. Nevertheless, also among women an inverse relationship was observed, with a relative rate of lung cancer of $0.40(95 \%$ CI $0.13-1.24$; trend- $p=0.101)$ for those in the highest quartile compared to the lowest quartile. After excluding cases occurring in the first year of follow-up, the inverse association between toenail selenium and lung cancer persisted (trend-p $=0.036$ ), with a relative rate of $0.52(95 \% \mathrm{Cl} 0.30-0.91)$ for those in the highest versus the lowest quintile (table 4). We also evaluated whether the association with toenail selenium was different among smoking categories (never, ex-, current smokers) by testing for interaction between smoking and toenail selenium. No significant interaction was noted, however (likelihood ratio $\chi^{2}=4.10, \mathrm{df}=8 ; \mathrm{p}=0.849$ ).

Table 4. Relative rate of lung cancer according to toenail selenium level in multivariate analysis*.

\begin{tabular}{|c|c|c|c|c|c|c|c|c|}
\hline \multirow{2}{*}{ Group } & \multirow{2}{*}{$\begin{array}{l}\text { No. of } \\
\text { cases }\end{array}$} & \multicolumn{5}{|c|}{ Quantile of toenail selenium level (boundaries in $\mu \mathrm{g} / \mathrm{g}$ ) } & \multicolumn{2}{|c|}{ Test for trend } \\
\hline & & $\begin{array}{l}1 \dagger \\
(\leq 0,483)\end{array}$ & $\begin{array}{l}2 \\
(\leq 0.530)\end{array}$ & $\begin{array}{l}3 \\
(\leq 0.573)\end{array}$ & $\begin{array}{l}4 \\
(\leq 0.630)\end{array}$ & $\begin{array}{l}5 \\
(>0.630)\end{array}$ & $x^{2}$ & (p-value) \\
\hline $\begin{array}{l}\text { All cases } \\
(95 \% \mathrm{CI})\end{array}$ & 317 & 1.00 & $\begin{array}{l}0.71 \\
(0.49-1.04)\end{array}$ & $\begin{array}{l}0.79 \\
(0.53-1.18)\end{array}$ & $\begin{array}{l}0.82 \\
(0.53-1.26)\end{array}$ & $\begin{array}{l}0.50 \\
(0.30-0.81)\end{array}$ & 7.697 & $(0.006)$ \\
\hline $\begin{array}{l}\text { Men } \\
\quad(95 \% \mathrm{CI})\end{array}$ & 285 & 1.00 & $\begin{array}{l}0.69 \\
(0.46-1.02)\end{array}$ & $\begin{array}{l}0.81 \\
(0.54-1.21)\end{array}$ & $\begin{array}{l}0.83 \\
(0.54-1.29)\end{array}$ & $\begin{array}{l}0.50 \\
(0.30-0.82)\end{array}$ & 6.415 & $(0.011)$ \\
\hline $\begin{array}{l}\text { Women } \ddagger \\
\qquad(95 \% \mathrm{CD})\end{array}$ & 32 & 1.00 & $\begin{array}{l}0.58 \\
(0.20-1.66)\end{array}$ & $\begin{array}{l}0.61 \\
(0.22-1.75)\end{array}$ & $\begin{array}{l}0.40 \\
(0.13-1.24)\end{array}$ & & 2.688 & $(0.101)$ \\
\hline $\begin{array}{l}\text { Excluding cases } \\
\text { from first yr of } \\
\text { follow-up } \\
(95 \% \mathrm{Cl})\end{array}$ & 228 & 1.00 & $\begin{array}{l}0.78 \\
(0.51-1.19)\end{array}$ & $\begin{array}{l}0.80 \\
(0.50-1.26)\end{array}$ & $\begin{array}{l}0.89 \\
(0.55-1.45)\end{array}$ & $\begin{array}{l}0.52 \\
(0.30-0.91)\end{array}$ & 4.380 & $(0.036)$ \\
\hline
\end{tabular}

- The model included terms for age, (gender), packyears of past smokers, packyears of current smokers, level of education.

$\uparrow$ Reference category.

\$ Because of the small number of cases, use was made of quartiles instead of quintiles; quartile boundaries were: $\leq 0.497, \leq 0.551, \leq 0.612,>0.612 \mu \mathrm{g} / \mathrm{g}$. 
As can be seen from table 5, the inverse association with toenail selenium is not restricted to a particular histological subtype of lung cancer. Because of the relatively small numbers, relative rates of lung cancer are presented here by quartile of toenail selenium. The relative rates (95\% CI in parentheses) for those in the highest toenail selenium quartile compared to the lowest quartile were $0.55(0.30-1.04)$ for squamous cell carcinoma, $0.19(0.04-0.87)$ for small cell carcinoma, $0.59(0.25-1.40)$ for adenocarcinoma, and $0.70(0.31-1.58)$ for large cell carcinoma and other types of lung carcinoma.

Table 5. Relative rate of lung cancer according to quartile of toenail selenium level in multivariate analysis", by histological subtype.

\begin{tabular}{|c|c|c|c|c|c|c|c|}
\hline \multirow{2}{*}{ Histology } & \multirow{2}{*}{$\begin{array}{l}\text { No. of } \\
\text { cases }\end{array}$} & \multicolumn{4}{|c|}{$\begin{array}{l}\text { Quartile of toenail selenium level } \\
\text { (boundaries in } \mu \mathrm{g} / \mathrm{g} \text { ) }\end{array}$} & \multicolumn{2}{|c|}{ Test for trend } \\
\hline & & $\begin{array}{l}1 \dagger \\
(\leq 0.497)\end{array}$ & $\begin{array}{l}2 . \\
(\leq 0.551)\end{array}$ & $\begin{array}{l}3 \\
(\leq 0.612)\end{array}$ & $\left.{ }^{4}>0.612\right)$ & $x^{2}$ & (p-value) \\
\hline $\begin{array}{l}\text { Squamous cell carc. } \\
\qquad(95 \% \text { CI) }\end{array}$ & 143 & 1.00 & $\begin{array}{l}0.95 \\
(0.60-1.50)\end{array}$ & $\begin{array}{l}0.84 \\
(0.50-1.42)\end{array}$ & $\begin{array}{l}0.55 \\
(0.30-1.04)\end{array}$ & 3.899 & $(0.048)$ \\
\hline $\begin{array}{l}\text { Small cell carc. } \\
\qquad(95 \% \mathrm{CI})\end{array}$ & 55 & 1.00 & $\begin{array}{l}0.71 \\
(0.32-1.56)\end{array}$ & $\begin{array}{l}1.59 \\
(0.76-3.30)\end{array}$ & $\begin{array}{l}0.19 \\
(0.04-0.87)\end{array}$ & 0.868 & $(0.351)$ \\
\hline $\begin{array}{l}\text { Adenocarcinoma } \\
\quad(95 \% \mathrm{CI})\end{array}$ & 62 & 1.00 & $\begin{array}{l}0.76 \\
(0.38-1.52)\end{array}$ & $\begin{array}{l}0.95 \\
(0.47-1.90)\end{array}$ & $\begin{array}{l}0.59 \\
(0.25-1.40)\end{array}$ & 0.987 & $(0.320)$ \\
\hline $\begin{array}{l}\text { Large cell, other } \\
\quad(95 \% \mathrm{CI})\end{array}$ & 57 & 1.00 & $\begin{array}{l}0.58 \\
(0.28-1.18)\end{array}$ & $\begin{array}{l}0.47 \\
(0.20-1.07)\end{array}$ & $\begin{array}{l}0.70 \\
(0.31-1.58)\end{array}$ & 2.046 & $(0.153)$ \\
\hline
\end{tabular}

- The model included terms for age, gender, packyears of past smokers, packyears of current smokers, level of education.

\$ Reference category.

In additional models we also adjusted for the intake of retinol, beta-carotene and vitamin $C$ in the subset of 293 cases and 2204 subcohort members who had both complete dietary and toenail data. This adjustment resulted in similar effect estimates (table 6, first line) as the model without adjustment for dietary variables. We also examined the association between toenail selenium and lung cancer by category of intake of these vitamins. Table 6 shows the relative rates, $95 \%$ confidence intervals and tests for trend for quintiles of toenail selenium in those who are in the lowest two quintiles and those who are in the highest two quintiles of intake of these three vitamins, respectively. The association between toenail selenium and lung cancer risk did not differ appreciably between those with a low or high retinol intake. In contrast, for beta-carotene and particularly for vitamin $\mathrm{C}$, the protective effect of selenium seems to be concentrated in those with a relatively low intake of these vitamins (trend$\mathrm{p}=0.028$ and $<0.001$ for the low beta-carotene and vitamin $\mathrm{C}$ groups, respectively). The relative rates (95\% CI in parentheses) of lung cancer for those in the highest quintiles of toenail selenium were $0.45(0.22-0.92)$ and $0.36(0.17-0.75)$ in the subjects with a low intake of beta-carotene and vitamin $C$, respectively. Tests for interaction indicated that the differences in estimates per selenium quintile between the low and high vitamin 
intake groups were not significant for beta-carotene (likelihood ratio test $\mathrm{p}=0.662$ ) or vitamin $\mathrm{C}(\mathrm{p}=0.439)$, but the compared groups are rather small.

Table 6. Relative rate of lung cancer according to toenail selenium level by category of intake of retinol, beta-carotene and vitamin $\mathrm{C}$.

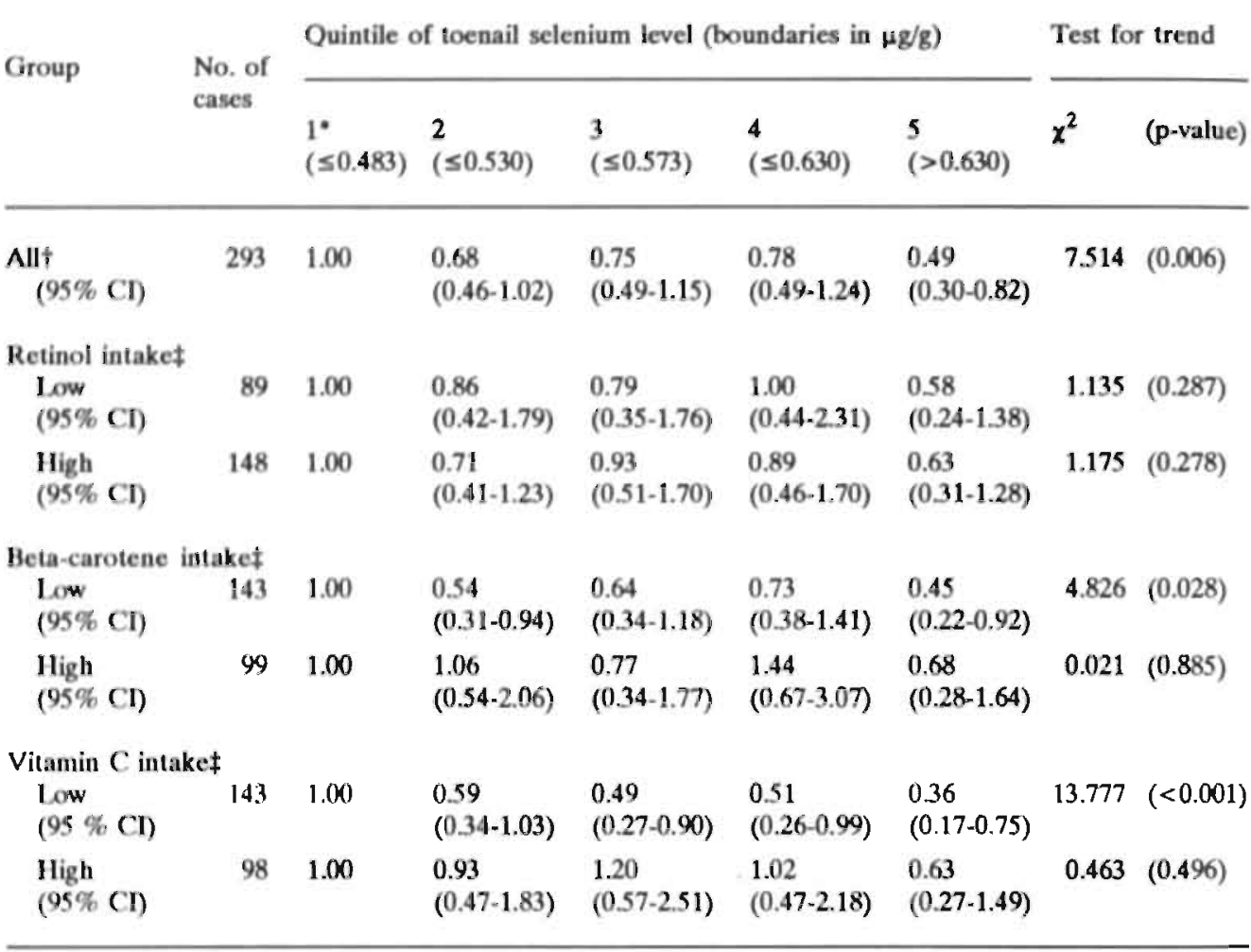

- Reícrence category.

† Model adjusted for age, gender, packyears of past smokers, packyears of current smokers, level of education, intake of retinol, beta-carotene and vitamin $\mathrm{C}$.

+ Low and high are defined as the two lowest quintiles and the two highest quintiles of intake, respectively.

\section{Discussion}

In this prospective cohort study, a statistically significant inverse trend was found between the toenail selenium level and the risk of lung cancer. A number of prospective studies on selenium in serum and lung cancer risk have been published, all using the nested case-control approach $(22,32)$. In the first prospective study that showed a significant inverse asscciation of selenium with total cancer, Willett et al. (22) found nonsignificantly lower serum selenium levels among lung cancer cases compared to controls. However, the difference between cases and controls was in fact greater for lung cancer than for all cancers combined. Because there were only 18 lung cancer cases involved, the association did not reach statistical significance. Salonen et al. did observe a significant inverse association between serum selenium and respiratory cancer risk (15 cases) in a Finnish cohort (26), whereas a nonsignificant inverse association with respiratory cancer (23 cases) was found in another Finnish cohort (27). 
Nonsignificantly lower serum selenium levels among lung cancer cases compared to controls have also been reported in four other studies $(24,28-30)$, but these nested casecontrol studies only included a small number of lung cancer cases. The largest nested case-control study on serum selenium and lung cancer to date was published by Knekt et al. (23). This Finnish cohort yielded 198 lung cancer cases and a strong, significant inverse association between serum selenium and lung cancer risk; the relative risk for men in the highest compared to the lowest quintille of serum selenium was estimated at 0.3 . In three other studies nonsignificantly positive associations between serum selenium and lung cancer have been observed $(25,31,32)$. The study by Menkes et al. (31) was the largest, involving 99 lung cancer cases. In that study " a positive association was also observed for the various histological subtypes of lung cancer.

Thus, in the majority of the studies an inverse association between serum selenium and lung cancer is observed, albeit mostly nonsignificant. It should be kept in mind that most of these studies were focused primarily on overall cancer risk and even then the number of cases was often rather small. The limited number of respiratory cancer cases in virtually all studies precludes the possibility to draw conclusions from the inconsistent findings, but in a number of these studies the association with selenium was stronger for respiratory cancer than for all cancers combined $(22-24,26,28,30)$. As has been noted before $(2,24)$, a possible explanation for the discrepant findings might be the difference in the range of selenium levels that has been investigated in the various countries. In

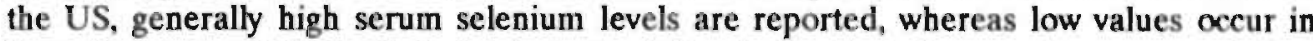
New Zealand and Finland. Indeed, most of the studies that show an inverse association originate from Finland where selenium intake used to be low (23). Selenium intake is moderate in the Netherlands and blood selenium levels are intermediate between those reported from New Zealand and the United States as is true for toenail selenium levels (33).

The inverse association between toenail selenium and lung eancer in our study was not restricted to men. This seems in apparent contrast with an earlier suggestion that the protective effect of selenium might be specific for men only (17). However, the size of most studies was not sufficient to allow site-specific analyses by gender. Genderspecific information is usually only reported for breast cancer; most studies have shown that there is no association between selenium and this type of cancer $(13,14,22-25,33)$. Because lung cancer often represents a substantial part of the male cases (in contrast to female cases) and breast cancer is the most important female cancer site, the suggested gender-specific effect of selenium might reflect site-specific effects. Indeed, as Coates et al. (24) have suggested, the differences in cancer site distributions between the various studies might also explain the inconsistent results of the published prospective studies.

As in other studies $(11,12)$, smoking was inversely related with toenail selenium levels in our population (34) and might therefore be an important confounder of the relationship between selenium and lung cancer. It is unlikely that there is major residual confounding by smoking in our analyses, however, because the inverse association persisted after presumably close control of smoking by using packyears of smoking. In addition, we found no clear indication that the selenium effect is restricted to specific subtypes of lung carcinoma that are associated more strongly with smoking such as squamous cell and small cell carcinoma (15).

Confounding by dietary variables was not observed after evaluating models that included terms for retinol, beta-carotene and vitamin $\mathrm{C}$ intake. (Because the Dutch food table does not contain information on the vitamin $E$ content of foods, we could not evaluate the influence of this vitamin.) Some evidence, however, was found for effect modification by the level of vitamin intake. While for beta-carotene and vitamin C the effect of a high toenail selenium level was concentrated in those with a relatively 
low intake of these vitamins, there was no clear effect modification by retinol intake, which is in line with the lower antioxidative capacity of retinol (35). Others have evaluated the interaction with antioxidant vitamins by studying serum levels of retinol, beta-carotene and $\alpha$-tocopherol; the interactions were only assessed with regard to overall cancer risk. While the effect modification by beta-carotene is supported by other studies $(23,32)$, the observations on interaction with retinol levels are inconsistent $(17,22-24,27,32)$. No other cohort studies on this subject have evaluated the effect modification by vitamin C. Nevertheless, since this vitamin can also protect against. oxidative damage, the observed interaction between selenium and vitamin $\mathbf{C}$ fits within the antioxidant hypothesis (36). It would be interesting to find out if a similar effect modification by vitamin $\mathrm{C}$ exists in the cohort studies that have been published. Vitamin $\mathrm{C}$ is, however, degraded rapidly in frozen serum (10), which is the probable reason why it has not been investigated in these cohort studies with nested case-control analyses. Therefore, the assessment of interaction will necessarily have to involve dietary vitamin $\mathrm{C}$ intake.

As in most serum studies regarding overall cancer risk $(22-24,27,30,31)$, although not all (29), we found no influence of preclinical lung cancer on the toenail selenium levels. This was indicated by the persistence of the inverse association after excluding cases detected in the first year of follow-up. Also, no increasing trend in the average toenail selenium levels was observed when cases were categorized by year of follow-up. This was to be expected since the toenail selenium level is assumed to be a long-term marker of selenium status, while the selenium level in serum is regarded as a short-term marker.

In conclusion, we observed an inverse association between toenail selenium and lung cancer risk. The association was not restricted to a particular gender, smoking category or histological subtype of lung cancer. The possible effect modification by beta-carotene and particularly vitamin $\mathrm{C}$ warrants further study.

\section{References}

1. Rotruck JT, Pope AI, Ganther HE, Swanson AB, Hafeman DG, Hoekstra WG. Selenium: biochemical role as a compenent of glutathione peroxidase. Science 1973; 179; 588-590.

2. Combs Gi. Seienium. In: Moon TE, Micozzi MS, eds, Nutrition and cancer prevention; investigating the role of micrenutrients. New York: Marcel Dekker, 1989: 389-420.

3. Clark L.C. Cantor KP, Allaway WII. Sclenium in forage crops and cancer mortality in U.S. counties. Arch Environ IJealth 1991; 46: 37-42.

4. Nakayama $T_{\text {, Kaneko }} \mathbf{M}$, Kodama $\mathbf{M}_{\text {n }}$ Nagata $\mathbf{C}$. Cigarette smoke induces DNA single-strand breaks in human cells. Nature $1985 ; 314: 462-464$.

5. Church DF, Pryor WA. Free-radical chemistry of cigarette smoke and its toxicological implications. Environ Health Perspect 1985; 64: 111-126.

6. Levander OA. Considerations on the assessment of selenium status. Fed Proc 1985; 44: $2579-2583$.

7. Broghamer WL, McConnell KP, Blotcky AL. Relationship between serum selenium levels and patients with carcinoma. Cancer 1976; 37: 1384-1388.

8. Willett WC, Stampfer MJ. Selenium and cancer. Br Med J 1988; 297: 373-374.

9. Morris JS, Stampfer MJ, Willett W. Dietary selenium in humans; toenails as an indicator. Biol Trace Elem Res 1983; 5: 529-537.

10. Willett W. Nutritional epidemiology. New York: Oxford University Press, 1990.

11. Hunter DJ, Morris IS, Chute $\mathrm{CG}$, et al. Predictors of selenium concentration in human toenails. An J Epidemiol 1990; 132: 114-122.

12. Swanson CA, Longnecker MP, Veillon $\mathbf{C}$, et al. Selenium intake, age, gender, and smoking in relation to indices of selenium status of adults residing in at seleniferous area. Am J Clin Nutr 1990; 52: 858-862.

13. Van Noord PA, Collette HJ, Maas MJ, de Waard F. Selenium levels in nails of premenopausal breast cancer patients assessed prediagnostically in a cohort-nested case-referent study among women screened in the DOM project. Int J Epidemiol 1987: 16(Suppl): 318-322. 
14. Hunter DJ, Morris JS, Stampfer MJ, Colditz GA, Speizer FE, Willett WC A prospective study of selenium status and breast cancer risk. JAMA 1990; 264: 1128-1131.

15. Lubin $\mathrm{JH}_{n}$ Blot WJ. Assessment of lung cancer risk factors by histologic category. J Natl Cancer Inst $1984 ; 73: 383-389$.

16. Stayner LT, Wegman DH. Smoking, occupation, and histopathology of lung cancer: a case-control study with the use of the Third National Cancer Survey. J Natl Cancer Inst 1983; 70; 421-426.

17. Kok FJ, de Bruijn AM, Hofman A, Vermeeren $\mathrm{R}$, Valkenburg HA. Is serum selenium a risk factor for cancer in men only? Am J Epidemiol 1987; 125: 12-16.

18. Van den Brandt PA, Goldbohm RA, van 't Veer P, Volovics A, Hermus RJ, Sturmans F. A large-scale prospective cohort study on diet and cancer in The Netherlands. J Clin Epidemiol 1990; 43: 285-295.

19. Van den Brandt PA, Schouten L, Goldbohm RA, Dorant E, Hunen PMII. Development of a record linkage protocol for use in the Dutch cancer registry for epidemiologicall research. Int I Epidemiol 1990; 19: 553-558.

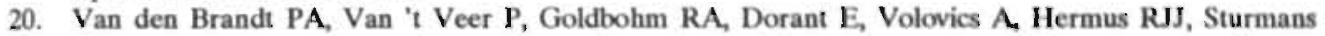
F. A prospective cohort study on dietary fat and the risk of postmenopausal breast cancer. Cancer Res (in press).

21. Self SG, Prentice RL. Asymptotic distribution theory and efficiency results for case-cohort studies. Ann Stat 1988; 16: 64-81.

22. Willett WC, Morris JS, Pressel S, et. al. Prediagnostic serum selenium and risk of cancer. Iance! 1983; ii: 130-134.

23. Knekt P, Aromaa A, Maatela J, et al. Serum selenium and subsequent risk of cancer among Finnish men and women. J Natll Cancer Inst 1990; 82: 864-868.

24. Coates RJ, Weiss NS, Daling JR, Morris JS, Labbe RF. Serum levels of selenium and retinol and the subsequent risk of cancer. Am J Epidemiol 1988; 128: 515-523.

25. Peleg I, Morris S, Hames CG. Is serum selenium a risk factor for cancer? Med Oncol Tumor Pharmacother 1985; 2 : 157-163.

26. Salonen JT, Salonein R, Lappetelainen R, Maenpaa PH, Alfthan G, Puska P. Risk of cancer in relation to serum concentrations of selenium and vitamins $A$ and $E$ : matched case-control anatysis of prospective data. Br Med J 1985; 290: 417-420.

27. Salonen JT, Alfthan G, Huttunen JK, Puska P. Association between serum selenium and the risk of cancer. Am J Epidemiol 1984; 120: 342-349.

28. Kok FJ, van Duyn CM, Hofman A, Vermeeren R, de Bruyn AM, Valkenburg HA. Micronutrients and the risk of lung cancer. N Engl J Med 1987; 316: 1416.

29. Virtamo J, Valkeila E, Alfthan G, Punsar S, Huttunen JK, Karvonen MJ. Serum selenium and rísk of cancer. A prospective follow-up of nine years. Cancer 1987; 60: 145-148.

30. Ringstad J, Jacobsen BK, Tretli S, Thomassen Y. Serum selenium conceniration associated with risk of cancer. J Cin Pathol 1988; 41: 454-457.

31. Menkes MS, Comstock GW, Vuilleumier JP, Helsing KJ, Rider AA, Brookmeyer R. Serum beta-carotene, vitamins $A$ and $E$, selenium, and the risk of lung cancer. N Engl J Med 1986; 315: 1250-1254.

32. Nomura A, Heilbrun IK, Morris JS, Stemmermann GN. Serum selenium and the risk of cancer, by specific sites: case-control analysis of prospective data. J Natl Cancer Inst 1987; 79: 103-108.

33. Van 't Veer P, van der Wielen RP, Kok FJ, Hermus RJ, Sturmans F. Selenium in diet, blood, and toenails in relation to breast cancer: a case-control study. Am J Epidemiol 1990; 131: 987-994.

34. Van den Brandt PA, Goldbohm RA, Van 't Veer P, Bode P, Hermus RJJ, Sturmans F. Predictors of toenail selenium levels in men and women. Cancer Epidemiol Biomark Prev (in press).

35. Halliwell B. How to characterize a biological antioxidant. Free Rad Res Comms 1990; 9: 1-32.

36. Bendich A, Machlin LJ, Scandurra O, Burton GW, Wayer DDM. The antioxidant role of vitamin C. Adv Free Radic Biol Med 1986; 2: 419-444. 



\section{Chapter 16}

\section{A prospective cohort study on toenail selenium and the risk of gastrointestinal cancer*}

Piet A. van den Brandt ${ }^{1}$, R. Alexandra Goldbohm ${ }^{1,2}$, Pieter van ' $t$ Veer $^{2}$, Peter Bode ${ }^{3}$, Elisabeth Dorant ${ }^{1}$, Rudolph J.J. Hermus ${ }^{2}$, Ferd Sturmans ${ }^{1}$.

1) Department of Epidemiology, University of Limburg, Maastrich, the Netherlands; 2) Department of Nutrition, TNO-Toxicology and Nutrition Institute, Zeist, the Netherlands; 3) Interfaculty Reactor Institute, Delft University of Technology, Delft, the Netherlands

\section{Abstract}

The assoclation between toenall selenium and the risk of stomach and colorectal cancer was investigated In a prospectlve cohort study on dlet and cancer, that started in the Netherlands in 1986 among 120,852 men and women aged 55.69 years. After 3.3 years of follow-up, 155, 313 and 166 microscopically confinned Incident cases of stomach, colon and rectum cancer were detected, respectively. Toenall selenium data were available for 104 stomach cancer, 234 colon cancer, 113 rectum cancer cases and 2459 members of a randomly selected subcohort. In a multivariate analysis, the relative rates of stomach cancer for subjects in increasing quintiles of toenall selenlum level were $1.00,0.44,0.59,0.84$ and 0.64 (trend $-\mathrm{p}=0.491$ ). For men, there was more evidence for an inverse association between toenail selenium and stomach cancer: the relative rate for those in the highest compared to the lowest quintile of toenail selenlum was 0.40 (95\% confidence interval, 0.17 to 0.96$)$, albeit that the test for trend was not significant $(p=0.136)$. For stomach cancer among women, there was no negative association with toenail selenium. The negative association between toenail selenium and stomach cancer was concentrated in subjects with a relatively low vitamin C intake. For beta-carotene, there was no evidence for such an effect modincation. Toenail selenium was not associated with the risk of colon or rectum cancer. After excluding cases diagnosed in the first year of follow-up, the relative rates of colon cancer for increasing quintlles of toenail selenium were 1.00, 1.27, 1.17, 0.75 and 1.07 (trend-p=0.554); for rectum cancer these relative rates were estimated at $1.00,1.73$, $0.83,1.58$ and 1.12 (trend $-\mathrm{p}=\mathbf{0 . 8 9 0}$ ). These data support a suggestlve, but inconsistent inverse association between selenium status and the risk of stomach cancer but not of colorectal cancer. 


\section{Introduction}

Animal studies (1) and ecologic studies among human populations (2-4) suggest that a low dietary selenium intake is associated with an increased risk of various types of cancer. Because the assessment of dietary selenium intake is unreliable (5), casecontrol and cohort studies on selenium and cancer have frequently used biologic markers of selenium status such as serum or toenail selenium levels. Case-control studies on serum selenium and cancer are difficult to interpret because serum levels might be reduced due to sequestration of selenium by tumor cells $(6,7)$. In patients with advanced upper gastrointestinal cancer serum selenium levels were also found to be progressively decreased (8). Prospective serum studies, in which this interpretation problem can be avoided, were mostly limited to overall cancer risk as the primary goal of the analysis (9-19). In all of these studies, a nested case-control approach was used. Although cases had significantly lower prediagnostic selenium levels than controls in various prospective studies $(9-11,13,16,19)$, the results are inconsistent. In a number of studies, however, the difference between cases and controls was greater for gastrointestinal cancer sites than for all cancer sites combined $(9,10,14,15,19)$. Apart from the study by Knekt et al. (19), the numbers of gastrointestinal cancer cases were usually too small to permit detailed analyses of these sites in the mentioned studies.

In recent years, toenail selenium has gained popularity as biomarker of selenium status, following observations that this marker is an indicator of long-term selenium status $(20,21)$ and reflects differences in selenium intake $(22,23)$. With regard to gastrointestinal cancer, one prospective study using toenail selenium has been reported. In this study among women, toenail selenium was not associated with colon cancer risk (24). The purpose of our study was to investigate the relationship between prediagnostic toenail selenium levels and the risk of stomach and colorectal cancer in a large-scale prospective cohort study among men and women in the Netherlands.

\section{Materials and methods}

\section{The cohort}

In September 1986, a prospective cohort study on diet and cancer of the breast, colon, rectum, stomach and lung, has been initiated in the Netherlands. The study design has been described in detail (25). The cohort included 58,279 men and 62,573 women aged 55-69 years at the start of the study. The study population originated from 204 municipal population registries throughout the country. At baseline, the cohort members completed a self-administered questionnaire on usual diet and potential confounding variables and also provided toenail clippings. The semi-quantitative food frequency questionnaire was specifically designed for, and pretested among men and women of the cohort age range and was validated against a 9-day dietary record method (Goldbohm et al., submitted for publication). For data processing and analysis the case-cohort approach is used: the cases are enumerated for the entire cohort (numerator information of incidence rates), while the accumulated person years of the entire cohort are estimated using a subcohort sample (providing the denominator information). Following this approach, a random subcohort of 3,500 subjects (1688 men, 1812 women) was sampled from the cohort after the baseline exposure measurement. The subcohort has been followed up for vital status information in order to estimate the accumulated persontime in the cohort. Until December 31, 1989 (the end of the present follow-up period), there were no subcohort members lost to follow-up. 


\section{Cancer follow-up}

Follow-up for incident cancer was accomplished by a computerized record linkage with all nine regional cancer registries in the Netherlands and with PALGA, the Dutch national data base of pathology reports. The method of record linkage has been published previously (26). Record linkage has been conducted annually with PALGA and the cancer registries. The lag time between diagnosis of cancer and definitive registration in the cancer registries is usually less than three months, but may occasionally extend to 1.5 years. Considering this lag time, the linkage performed in 1991 thus accounted for presumably all cancers diagnosed until the end of 1989. The analysis in this report is restricted to the cancer incidence in the period from September 17, 1986 (cohort baseline measurement) until December 31, 1989, i.e. a follow-up period of 3.3 years. In this period a total of 1882 cases of breast, colorectal, stomach or lung cancer were detected in the cohort of 120,852 subjects. The cancer follow-up was estimated to be $95 \%$ complete after comparing observed and expected numbers of incident cases in this follow-up period (27).

\section{Population for analysis}

Among the 1882 cases, there were 176 stomach, 351 colon and 185 rectum cancei cases. After excluding cases reporting a history of cancer other than skin cancer in the baseline questionnaire (18 stomach, 39 colorectal cancer cases), cases with in situ carcinoma (2 stomach, 16 colorectal cancer cases) and cases without a microscopically confirmed diagnosis ( 0 stomach $_{\|} 2$ colorectal cancer cases), 155 incident stomach cancer and 479 colorectal cancer cases were available for analysis. After excluding prevalent cancer cases other than skin cancer from the subcohort of 3500 as well, 3346 subjects remained in this group. Toenail clippings had been provided by 107 stomach cancer cases, 361 colorectal cancer cases and 2569 subcohort members. Problems with the detection of toenail selenium (interference by other elements such as calcium) occurred in 1,5 and 16 of these 107,361 and 2569 specimens, respectively. Another 2, 9 and 94 specimens were excluded from the stomach cancer, colorectal cancer and subcohort groups, respectively, because the specimens weighed less than $10 \mathrm{mg}$, which would yield unreliable selenium measurements. Thus, toenail selenium data on 104 stomach cancer ( 84 men, 20 women), 234 colon cancer (121 men, 113 women), 113 rectum cancer (77 men, 36 women), and 2459 subcohort members ( 1211 men, 1248 women) were available for analysis.

\section{Determination of toenail selenium levels}

The toenail selenium analyses were canied out by the Interfaculty Reactor Institute (IRI) at Delft University, the Netherlands. Each analytical batch contained toenail specimens of cases and subcohort members, and specimens were analyzed in a manner blinded with respect to case/subcohort status. Toenails were first cleared by scratching off any debris with a quartz knife. After ultrasonic cleaning with acetone for 15 minutes, distilled water for 10 minutes and acetone for 15 minutes, respectively, the specimens were freeze-dried during 15 hours to eliminate any humidity variations between runs. The selenium content of the toenails was measured by instrumentai neutron activation analysis of the metastable-selenium-77 isotope. The specimens were irradiated for 17 seconds in a thermal flux of $1.2 \times 10^{13}$ neutrons. $\mathrm{s}^{-1} \cdot \mathrm{cm}^{-2}$. After a decay time of 20 seconds, gamma radiation of ${ }^{77 \mathrm{~m}} \mathrm{Se}$ was measured for 60 seconds. The accuracy of the method was checked by analysis of a certified Bovine liver standard (Standard Reference Material 1577a of the US National Bureau of Standards). For 26 determinations, a mean value $( \pm$ SD) of $0.70 \pm 0.04 \mu \mathrm{g} / \mathrm{g}$ selenium was observed against. a certified value of $0.71 \pm 0.04 \mu \mathrm{g} / \mathrm{g}$. The precision of the method was evaluated 
by duplicate selenium measurements of specimens from 27 randomly selected subjects; the coefficient of variation was 6.6 percent.

\section{Data analysis}

The relationship between toenail selenium level and its various potential predictors has been analyzed before (28). For each of the three sites (stomach, colon, rectum cancer) the gender-specific mean toenail selenium levels of the cases were compared with those of the subcohort. To evaluate the potential influence of prediagnostic cancer on toenail selenium levels, the cases were categorized according to the year of follow-up in which the diagnosis was made. For each site, the mean toenail sellenium concentrations of the case groups defined by year of follow-up were then compared with each other.

Next, case-cohort analyses (29) were conducted, based on the assumption that survival times were exponentially distributed in this follow-up period (Volovics et al., in preparation). For these analyses, toenail selenium levels were categorized into quartiles or quintiles (depending on the available number of cases) according to the distribution in the subcohort. In the gender-age-stratified analyses, we computed Mantel-Haensze! relative rates of stomach, colon and rectum cancer for each of the quantiles, $95 \%$ confidence intervals, and tests for trend in the relative rates (which were corrected for the additional variance introduced by the subcohort sampling). In the multivariate casecohori analyses, relative rates (with corrected $95 \%$ confidence intervals) of cancer were computed after adjustment for the effects of several factors simultaneously. For stomach cancer, these included gender, age, smoking (expressed as packyears for past and current smokers), highest levell of education (categorized as low/medium/high) and intake of vitamin $\mathrm{C}$ and beta-carotene. For colon and rectum cancer, adjustment was made for gender, age, family history of intestinal cancer, Quetelet index, level of education and alcohol use. In addition to these factors, we also fitted models with additional adjustment for the intake of calories, fat, protein, carbohydrate, fiber, betacarotene and vitamin $\mathrm{C}$; since this additional adjustment did not yield materially different results, we only present the former, simpler models. Similarly, for the stomach cancer analyses the additional adjustment for family history of stomach cancer did not affect the relative rate estimates regarding selenium. For each analysis, tests for trend were computed based on likelihood ratio tests with scores of 1-5 assigned to increasing quintiles of toenail selenium, as in the stratified analysis. Apart from analyses for the total group, analyses were also conducted for men and women separately, and after excluding cases occurring in the first year of follow-up. Two-sided p-values are used throughout this report. The analyses were carried out using the GLIM statistical package $(30,31)$.

\section{Results}

In table 1 the average toenail selenium levels among men and women in each of the case groups are presented. For the subcohort the mean $( \pm S D)$ toenail selenium concentrations were $0.547( \pm 0.126) \mu \mathrm{g} / \mathrm{g}$ in men and $0.575( \pm 0.109) \mu \mathrm{g} / \mathrm{g}$ in women. Male stomach cancer cases showed lower selenium levels than men in the subcohort while female stomach cancer cases showed higher levels than female subcohort members. For colon cancer these differences had the same directions but were less marked. Rectum cancer cases showed higher toenail selenium levels than subcohort members in men and women. When cases were categorized with respect to the year of follow-up in which they were diagnosed, there was no trend towards lower toenail selenium levels in cases occurring closer to baseline in the stomach and rectum cancer 
group, indicating no effect of prediagnostic cancer on toenail selenium levels for these sites. For colon cancer, however, the lower selenium levels among cases occurring in the first year of follow-up suggest the presence of such an effect for this site (table 1).

Table 1. Toenail selenium levels ( $\mu \mathrm{g} / \mathrm{g}$ ) in cases according to gender and year of follow-up.

\begin{tabular}{|c|c|c|c|c|c|c|}
\hline \multirow[t]{2}{*}{ Group* } & \multicolumn{2}{|c|}{ Stomach cancer } & \multicolumn{2}{|c|}{ Colon cancer } & \multicolumn{2}{|c|}{ Rectum cancer } \\
\hline & $\mathrm{D}$ & Mean $\pm S D$ & n & Mean $\pm S D$ & n & Mean $\pm S D$ \\
\hline \multicolumn{7}{|l|}{ Gender } \\
\hline Men & 84 & $0.528 \pm 0.081$ & 121 & $0.536 \pm 0.092$ & 77 & $0.596 \pm 0.410$ \\
\hline Women & 20 & $0.647 \pm 0.184$ & 113 & $0.561 \pm 0.104$ & 36 & $0.580 \pm 0.089$ \\
\hline \multicolumn{7}{|c|}{ Year of follow-up } \\
\hline 1 & 30 & $0.566 \pm 0.142$ & 69 & $0.519 \pm 0.945$ & 31 & $0.561 \pm 0.125$ \\
\hline 2 & 31 & $0.543 \pm 0.127$ & 69 & $0.559 \pm 0.108$ & 39 & $0.632 \pm 0.542$ \\
\hline 3 & 31 & $0.548 \pm 0.088$ & 80 & $0.563 \pm 0.092$ & 31 & $0.591 \pm 0.190$ \\
\hline 4 & 12 & $0.543 \pm 0.099$ & 16 & $0.546 \pm 0.082$ & 12 & $0.534 \pm 0.131$ \\
\hline
\end{tabular}

- Mean $( \pm$ SD) selenium levels in the subcohort were $0.547( \pm 0.126)$ for men $(n=12.11)$ and 0.575 $( \pm 0.109) \mu \mathrm{g} / \mathrm{g}$ for women $(\mathrm{n}=1248)$.

The results of the gender-age-stratified analyses are presented in table 2 , for each of the three sites. This table shows, per quintile of toenail selenium, the number of cases observed in the cohort and the person years that were accumulated by the subcohort members.

Table 2. Mantel-Haenszel relative rate of stomach, colon and rectum eancer according to toenail selenium level in gender-age-stratified analysis.

\begin{tabular}{|c|c|c|c|c|c|c|c|}
\hline \multirow{2}{*}{ Tumor site } & \multicolumn{5}{|c|}{ Quintile of toenail selenium leve! (boundaries in $\mu \mathrm{g} / \mathrm{g}$ ) } & \multicolumn{2}{|c|}{ Test for trend } \\
\hline & $\begin{array}{l}1^{*} \\
(\leq 0.483)\end{array}$ & $\begin{array}{l}2 \\
(\leq 0.530)\end{array}$ & $\begin{array}{l}3 \\
(\leq 0.573)\end{array}$ & $\begin{array}{l}4 \\
(\leq 0.630)\end{array}$ & $\begin{array}{l}5 \\
(>0.630)\end{array}$ & $x^{2}$ & (p-value) \\
\hline \multicolumn{8}{|l|}{ Stomach } \\
\hline $\begin{array}{l}\text { No. of cases } \\
\text { (Person years } \\
\text { in subcohort) }\end{array}$ & $\begin{array}{l}32 \\
(1599)\end{array}$ & $\begin{array}{l}19 \\
(1596)\end{array}$ & $\begin{array}{l}17 \\
(1593)\end{array}$ & $\begin{array}{l}19 \\
(1588)\end{array}$ & $\begin{array}{l}17 \\
(1610)\end{array}$ & & \\
\hline $\begin{array}{l}\mathrm{RR}_{\mathrm{MH}} \\
(95 \% \mathrm{CI})\end{array}$ & 1.00 & $\begin{array}{l}0.69 \\
(0.38-1.25)\end{array}$ & $\begin{array}{l}0.641 \\
(0.35-1.17)\end{array}$ & $\begin{array}{l}0.83 \\
(0.45-1.53)\end{array}$ & $\begin{array}{l}0.61 \\
(0.33-1.11)\end{array}$ & 1.272 & $(0.259)$ \\
\hline \multicolumn{8}{|l|}{ Colon } \\
\hline $\begin{array}{l}\text { No. of cases } \\
\text { (Person years } \\
\text { in subcohort) }\end{array}$ & $\begin{array}{l}54 \\
(1599)\end{array}$ & $\begin{array}{l}53 \\
(1590)\end{array}$ & $\begin{array}{l}48 \\
(1592)\end{array}$ & $\begin{array}{l}36 \\
(1588)\end{array}$ & $\begin{array}{l}43 \\
(1607)\end{array}$ & & \\
\hline $\begin{array}{l}\mathrm{RR}_{\mathrm{MH}} \\
(95 \% \mathrm{Cl})\end{array}$ & 1.00 & $\begin{array}{l}0.98 \\
(0.65-1.48)\end{array}$ & $\begin{array}{l}0.89 \\
(0.58-1.36)\end{array}$ & $\begin{array}{l}0.68 \\
(0.43-1.08)\end{array}$ & $\begin{array}{l}0.77 \\
(0.49-1.19)\end{array}$ & 2.713 & $(0.100)$ \\
\hline \multicolumn{8}{|l|}{ Rectum } \\
\hline $\begin{array}{l}\text { No. of cases } \\
\text { (Person years } \\
\text { in subcohort) }\end{array}$ & $\begin{array}{l}25 \\
(1598)\end{array}$ & $\begin{array}{l}28 \\
(1595)\end{array}$ & $\begin{array}{l}13 \\
(1591)\end{array}$ & $\begin{array}{l}25 \\
(1588)\end{array}$ & $\begin{array}{l}22 \\
(1610)\end{array}$ & & \\
\hline $\begin{array}{l}\mathrm{RR}_{\mathrm{MH}} \\
(95 \% \mathrm{CI})\end{array}$ & 1.00 & $\begin{array}{l}1.20 \\
(0.68-2.12)\end{array}$ & $\begin{array}{l}0.61 \\
(0.30-1.23)\end{array}$ & $\begin{array}{l}1.20 \\
(0.67-2.14)\end{array}$ & $\begin{array}{l}1.01 \\
(0.55-1.84)\end{array}$ & $<0.001$ & $(0.991)$ \\
\hline
\end{tabular}

- Reference category. 
In the calculation of the person years at risk, it was assumed that subjects who develop a certain cancer are still at risk for cancer at a different site. It can be seen from table 2 that the Mantel-Haenszel relative rates of stomach and colon cancer are nonsignificantly decreased for subjects in the upper quintiles of toenail selenium (pvalues for trend-test for stomach and colon were 0.259 and 0.100 , respectively). For rectum cancer there was no association with toenail selenium in the gender-agestratified analysis (trend- $p=0.991$ ).

In the multivariate analysis regarding, stomach cancer we adjusted for gender, age, smoking, education and the intake of vitamin $\mathrm{C}$ and beta-carotene. When all the 92 cases with complete data on the variables in the model were used in the analysis "there was some evidence for a decreased risk of stomach cancer, with relative rates of 1.00 , $0.44,0.59,0.84$ and 0.64 for increasing quintiles of toenail selenium, but the test for trend was not significant $(p=0.491)$. Only for those in the second quintile the relative rate was significantly different from unity. (A model in which vitamin $C$ and betacarotene were replaced by the intake of vegetables and fruits yielded essentially similar results.) The negative association between stomach cancer and toenail selenium was only seen in men, where the relative rates for increasing quintiles of toenail selenium were $1.00,0.49,0.50,0.92$ and 0.40 , respectively. Although the relative rates for those in the second and fifth quintiles compared to the lowest quintile were significantly different from one, the test for trend was not significant (trend-p=0.136). In women, no evidence for a negative association was found. A more definitive evaluation of a possible modest positive association in women requires more cases than the 20 who were available now. The relative rate estimates were not materially affected after exclusion of the cases occurring in the first year of follow-up, indicating no effect of prediagnostic stomach cancer on toenail selenium levels (table 3 ).

Table 3. Relative rate of stonach cancer according to toenail selenium level in multivariate analysis*.

\begin{tabular}{|c|c|c|c|c|c|c|c|c|}
\hline \multirow{2}{*}{ Tumor site } & \multirow{2}{*}{$\begin{array}{l}\text { No. of } \\
\text { cases }\end{array}$} & \multicolumn{5}{|c|}{ Quantile of toenail selenium level (boundaries in $\mu \mathrm{g} / \mathrm{g}$ ) } & \multicolumn{2}{|c|}{ Test for trend } \\
\hline & & $\begin{array}{l}1 \dagger \\
(\leq 0.483)\end{array}$ & $\begin{array}{l}2 \\
(\leq 0.530)\end{array}$ & $\begin{array}{l}3 \\
(\leq 0.573)\end{array}$ & $\begin{array}{l}4 \\
(\leq 0.630)\end{array}$ & $\begin{array}{l}5 \\
(>0.630)\end{array}$ & $x^{2}$ & (p-value) \\
\hline $\begin{array}{l}\text { All cases } \\
\qquad(95 \% \mathrm{CI})\end{array}$ & 92 & 1.00 & $\begin{array}{l}0.44 \\
(0.22-0.88)\end{array}$ & $\begin{array}{l}0.59 \\
(0.31-1.15)\end{array}$ & $\begin{array}{l}0.84 \\
(0.44-1.61)\end{array}$ & $\begin{array}{l}0.64 \\
(0.33-1.27)\end{array}$ & 0.474 & $(0.491)$ \\
\hline $\begin{array}{l}\text { Men } \\
\quad(95 \% \mathrm{Cl})\end{array}$ & 72 & 1.00 & $\begin{array}{l}0.49 \\
(0.24-0.99)\end{array}$ & $\begin{array}{l}0.50 \\
(0.24-1.06)\end{array}$ & $\begin{array}{l}0.92 \\
(0.46-1.82)\end{array}$ & $\begin{array}{l}0.40 \\
(0.17-0.96)\end{array}$ & 2.225 & $(0.136)$ \\
\hline $\begin{array}{l}\text { Women } \$ . \\
(95 \% \mathrm{CI})\end{array}$ & 20 & 1.00 & $\begin{array}{l}0.73 \\
(0.14-3.71)\end{array}$ & $\begin{array}{l}1.36 \\
(0.33-5.60)\end{array}$ & $\begin{array}{l}1.68 \\
(0.43-6.54)\end{array}$ & & 1.305 & $(0.253)$ \\
\hline $\begin{array}{l}\text { Frcluding cases } \\
\text { from first yr of } \\
\text { follow-up } \\
(95 \% \mathrm{Cl})\end{array}$ & 67 & 1.00 & $\begin{array}{l}0.47 \\
(0.21-1.02)\end{array}$ & $\begin{array}{l}0.56 \\
(0.26-1.22)\end{array}$ & $\begin{array}{l}0.91 \\
(0.43-1.91)\end{array}$ & $\begin{array}{l}0.60 \\
(0.27-1.34)\end{array}$ & 0.440 & $(0.507)$ \\
\hline
\end{tabular}

- The model included ierms for age, (gender), packyears of past smokers, packyears of current smokers, level of education, intake of beta-carotene and vitamin $C$.

$\dagger$ Reference category.

+ Because of the small number of cases, use was made of quartiles instead of quintiles; quartile boundaries were: $\leq 0.497, \leq 0.551, \leq 0.612,>0.612 \mu \mathrm{g} / \mathrm{g}$. 
The results for colon cancer and rectum cancer are presented in a similar way in tables 4 and 5 , respectively. There seems to be some evidence for a negative relationship between toenail selenium and the risk of colon cancer in the analysis among all 216 cases with complete data (trend- $p=0.067$ ); this association is seen in both men and women. However, (as already expected from the results in table 1) the negative association disappeared when cases occurring in the first year of follow-up are excluded: the relative rates of colon cancer in that model were 1.00,1.27, 1.17, 0.75 and 1.07 for increasing quintiles (trend- $\mathrm{p}=0.554$ ) (table 4 ). With regard to rectum cancer, no association was observed with toenail selenium concentration when all cases were considered; the relative rates for increasing quintiles of toenail selenium were $1.00,1.13$, $0.58,1.19$ and 1.05 , respectively (trend-p $=0.829$ ). There was also no evidence for an association in gender-specific analyses or when cases from the first year of follow-up were excluded.

Table 4. Relative rate of colon cancer according to toenail selenium level in multivariate analysis".

\begin{tabular}{|c|c|c|c|c|c|c|c|c|}
\hline \multirow{2}{*}{ Tumor site } & \multirow{2}{*}{$\begin{array}{l}\text { No. of } \\
\text { cases }\end{array}$} & \multicolumn{5}{|c|}{ Quintile of toenail selenium level (boundaries in $\mu \mathrm{g} / \mathrm{g}$ ). } & \multicolumn{2}{|c|}{ Test for trend } \\
\hline & & $\begin{array}{l}1 \dagger \\
(\leq 0.483)\end{array}$ & $\begin{array}{l}2 \\
(\leq 0.530)\end{array}$ & $\begin{array}{l}3 \\
(\leq 0.573)\end{array}$ & $\begin{array}{l}4 \\
(\leq 0.630)\end{array}$ & $\begin{array}{l}5 \\
(>0.630)\end{array}$ & $x^{2}$ & (p-value) \\
\hline $\begin{array}{l}\text { All cases } \\
\qquad(95 \% \mathrm{CI})\end{array}$ & 216 & 1.00 & $\begin{array}{l}1.08 \\
(0.71-1.63)\end{array}$ & $\begin{array}{l}0.89 \\
(0.56-1.40)\end{array}$ & $\begin{array}{l}0.67 \\
(0.40-1.12)\end{array}$ & $\begin{array}{l}0.80 \\
(0.50-1.29)\end{array}$ & 3.353 & $(0.067)$ \\
\hline $\begin{array}{l}\text { Men } \\
\quad(95 \% \mathrm{CI})\end{array}$ & 116 & 1.00 & $\begin{array}{l}1.20 \\
(0.70-2.05)\end{array}$ & $\begin{array}{l}1.06 \\
(0.59-1.90)\end{array}$ & $\begin{array}{l}0.85 \\
(0.45-1.60)\end{array}$ & $\begin{array}{l}0.82 \\
(0.43-1.58)\end{array}$ & 0.965 & $(0.326)$ \\
\hline $\begin{array}{l}\text { Women } \\
\qquad(95 \% \mathrm{CI})\end{array}$ & 100 & 1.00 & $\begin{array}{l}0.94 \\
(0.49 \ldots 1.81)\end{array}$ & $\begin{array}{l}0.71 \\
(0.37-1.38)\end{array}$ & $\begin{array}{l}0.51 \\
(0.25-1.04)\end{array}$ & $\begin{array}{l}0.77 \\
(0.41-1.45)\end{array}$ & 2.287 & $(0.131)$ \\
\hline $\begin{array}{l}\text { Excluding cases } \\
\text { from first yr of } \\
\text { follow-up } \\
(95 \% \text { Ci) }\end{array}$ & 150 & 1.00 & $\begin{array}{l}1.27 \\
(0.76-2.12)\end{array}$ & $\begin{array}{l}1.17 \\
(0.67-2.02)\end{array}$ & $\begin{array}{l}0.75 \\
(0.40-1.43)\end{array}$ & $\begin{array}{l}1.07 \\
(0.61-1.88)\end{array}$ & 0.350 & $(0.554)$ \\
\hline
\end{tabular}

* The model included terms for age, (gender), familial intestinall cancer, level of education, Quetelet index, alcohol use.

$\dagger$ Reference category.

Because we have observed a modification of the effect of selenium by the level of intake of vitamin $\mathrm{C}$ and beta-carotene in a previous study on toenail sejenium and lung cancer (Van den Brandt et al., submitted for publication), we evaluated this possibility in the current study also for stonach cancer. The resuits are given in table 6 where the relative rates of stomach cancer per quartile (because of the small number of cases) of toenail selenium are presented for subjects with relatively low and high intake of each of the two vitamins, respectively. Only for vitamin $C$, there was some evidence of effect modification: the negative association between selenium and stomach cancer was concentrated in the low vitamin $\mathrm{C}$ group, no statistical significance was reached. Among subjects with a relatively high vitamin $C$ intake the association between selenium and stomach cancer was inconsistent. For beta-carotene no indication for effect modification 
was found, although the effect estimates were somewhat lower in the low than in the high beta-carotene group.

Table 5. Relative rate of rectum cancer according to toenail selenium level in multivariate analysis*

\begin{tabular}{|c|c|c|c|c|c|c|c|c|}
\hline \multirow{2}{*}{ Tumor site } & \multirow{2}{*}{$\begin{array}{l}\text { No. of } \\
\text { cases }\end{array}$} & \multicolumn{5}{|c|}{ Quantile of toenail selenium level (boundaries in $\mu \mathrm{g} / \mathrm{g}$ ) } & \multicolumn{2}{|c|}{ Test for trend } \\
\hline & & $\begin{array}{l}1+ \\
(\leq 0.483)\end{array}$ & $\begin{array}{l}2 \\
(\leq 0.530)\end{array}$ & $\begin{array}{l}3 \\
(\leq 0.573)\end{array}$ & $\begin{array}{l}4 \\
(\leq 0.630)\end{array}$ & $\begin{array}{l}5 \\
(>0.630)\end{array}$ & $x^{2}$ & (p-value) \\
\hline $\begin{array}{l}\text { All cases } \\
(95 \% \mathrm{CI})\end{array}$ & 102 & 1.00 & $\begin{array}{l}1.13 \\
(0.61-2.07)\end{array}$ & $\begin{array}{l}0.58 \\
(0.27-1.25)\end{array}$ & $\begin{array}{l}1.19 \\
(0.60-2.35)\end{array}$ & $\begin{array}{l}1.05 \\
(0.54-2.03)\end{array}$ & 0.047 & $(0.829)$ \\
\hline $\begin{array}{l}\text { Men } \\
\qquad(95 \% \mathrm{Cl})\end{array}$ & 70 & 1.00 & $\begin{array}{l}1.30 \\
(0.66-2.56)\end{array}$ & $\begin{array}{l}0.66 \\
(0.28-1.57)\end{array}$ & $\begin{array}{l}1.16 \\
(0.55-2.45)\end{array}$ & $\begin{array}{l}0.91 \\
(0.41-2.00)\end{array}$ & 0.116 & $(0.733)$ \\
\hline $\begin{array}{l}\text { Women } \ddagger \\
(95 \% \mathrm{CI})\end{array}$ & 32 & 1.00 & $\begin{array}{l}0.82 \\
(0.27-2.51)\end{array}$ & $\begin{array}{l}0.44 \\
(0.12-1.61)\end{array}$ & $\begin{array}{l}1.58 \\
(0.59-4.22)\end{array}$ & & 1.204 & $(0.273)$ \\
\hline $\begin{array}{l}\text { Excluding cases } \\
\text { from first yr of } \\
\text { follow-up } \\
(95 \% \mathrm{CD})\end{array}$ & 76 & 1.00 & $\begin{array}{l}1.73 \\
(0.85-3.51)\end{array}$ & $\begin{array}{l}0.83 \\
(0.34-2.01)\end{array}$ & $\begin{array}{l}1.58 \\
(0.71-3.51)\end{array}$ & $\begin{array}{l}1.12 \\
(0.49-2.55)\end{array}$ & 0.019 & $(0.890)$ \\
\hline
\end{tabular}

- The model includedi terms for age, (gender), familial intestinal cancer, level of education, Quetelet index, alcohol use.

$\uparrow$ Reference category.

¥ Because of the small number of cases, use was made of quartiles instead of quintiles; quartile boundaries were: $\leq 0.497, \leq 0.551, \leq 0.612,>0.612 \mu \mathrm{g} / \mathrm{g}$.

Table 6. Relative rate of stomach cancer according to toenail selenium level by category of intake of beta-carotene and vitamin $C$.

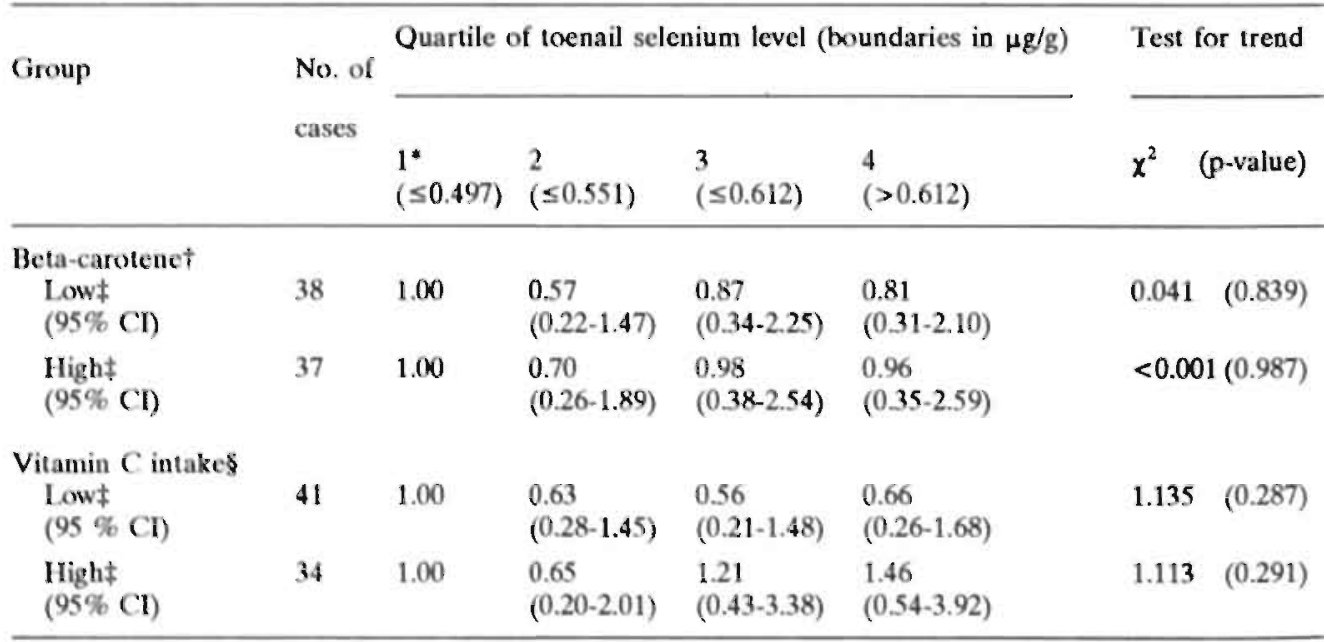

- Reference category.

† Model adjusted for age, gender, packyears of past smokers, packyears of current smokers, level of education, vitamin $\mathrm{C}$ intake.

\$ Low and high are defined as the two lowest quintiles and the two highest quintiles of intake, respectively.

$\$$ Model adjusied for age, gender, packyears; of past smokers, packyears of current smokers, level of education, beta-carotene intake. 


\section{Discussion}

In this prospective study we found suggestive, but inconsistent evidence for a negative association between toenail selenium and the risk of stomach cancer, but not for colon (after excluding cases from the first year of follow-up) or rectum cancer. The negative association between toenail selenium and stomach cancer was only observed in men. The number of female cases was too small to reach a more definitive conclusion about the possible relationship.

Few prospective studies have been carried out on selenium status and the risk of cancer of gastrointestinal sites. With regard to toenail selenium, only one other study has been reported. In that prospective study among over 14,000 Dutch women, 36 cases of colorectal cancer were observed after a mean follow-up of 5.8 years (24). As in our study, there was no evidence of an association with colorectal cancer in that study. With regard to serum selenium, a number of nested case-control studies have yielded results specifically for gastrointestinal cancers, albeit mostly for all cancers of the digestive tract combined $(9-12,14,15,17-19,32-34)$. Unfortunately, most of these studies only present information on the overall difference in mean selenium levels between cases and controls, and no information on relative risks per quantile of serum selenium (due to the small number of cases). Based on the differences in mean serum selenium level between cases and controls, in several studies the association with selenium was stronger for gastrointestinal cancer than for total cancer $(9,10,14,15,19)$. In three other studies, the association with selenium was less strong for gastrointestinal sites than for all cancers combined $(11,12,18)$. In six reports $(14,15,19,32-34)$ information is presented for specific sites within the digestive tract. A negative association with serum selenium was found for pancreatic cancer $(19,34)$, stomach cancer $(19,32)$ and oesophageal and stomach cancer combined (32), while no (significant) association was found for colorectal cancer in four studies $(14,19,32,33)$. The reports by Knekt et al. $(19,32)$ represent the largest prospective study to date on serum selenium and the risk of gastrointestinal cancer. In that Finnish cohort, a significant association between serum selenium and stomach cancer was found among men, with a relative risk for men in the upper four quintiles compared to the lowest quintile of serum selenium of 0.14 , after a median 8-year follow-up. For women, a nonsignificant negative association was found, with a corresponding relative risk of 0.28 . Thus, while our observation of a negative association between toenail selenium and stomach cancer among men is supported by the study of Knekt et al. (19), the findings among women seem to be in contrast with that report. However, our result among women is based on only 20 cases; a larger number of cases is needed for a more definitive conclusion. For both colon and rectum cancer, our observations of no effect are in agreement with the earlier studies using serum selenium $(14,19,32,33)$ and toenail selenium (24).

Whereas the toenail selenium levels seemed to be decreased by prediagnostic colon cancer in our study, this was not observed for stomach cancer. Although absence of an effect of prediagnostic cancer has been reported often in the serum studies with regard to overall cancer $(9,10,17-19)$, there are very few studies reporting site-specific analyses in this respect. Nevertheless, our findings with regard to effects of prediagnostic stomach and colon cancer are supported by the results of the serum studies by Knekt et al. (19) and the toenail study by Van Noord (24). Thus, case-control studies on selenium status and colon cancer need to be interpreted with caution.

Following the observation that smoking is negatively associated with toenail selenium levels (Van den Brandt et al., submitted for publication), we cannot exclude a possible confounding effect of smoking on the association between toenail selenium and stomach cancer. However, we have attempted to control for smoking in our analysis by 
using packyears of smoking instead of a crude categorization into never/ex/current smokers; the negative association persisted after this tighter control for smoking.

In our previous study we observed that the negative association between toenail selenium and lung cancer was modified by the level of intake of the antioxidants (35), beta-carotene and particularly vitamin C (Van den Brandi et al., submitted for publication). For stomach cancer, we also found some evidence for an effect. modification by vitamin $\mathrm{C}$ intake but it is less strong than for lung cancer. Because of the differences in etiology of stomach cancer and lung cancer, it is difficult to extrapolate from one site to the other. The discrepancies between the current finding. with regard to selenium may be partly explained by the fact that smoking, is a less important risk factor for stomach cancer than for lung cancer. Since smoking induces. oxidative stress $(36,37)$, the effects of antioxidants and the interaction between them (e.g., selenium and vitamin C) may be more evident for lung cancer than for stomach cancer. For stomach cancer, the interaction between selenium and vitamin $\mathrm{C}$ may also be of a different nature since the protective effect of vitamin $\mathrm{C}$ against this cancer may have to do with its role in blocking the formation of $\mathrm{N}$-nitroso compounds in gastric conditions (38-40), which is independent of its antioxidative capacity.

In conclusion, we found a suggestive, but inconsistent negative association between toenail selenium level and the risk of stomach cancer. The effect seemed only to be present in men and there was some evidence for an effect modification by vitamin $\mathrm{C}$. However, the number of cases is still rather small; a more definitive evaluation would require a longer follow-up period. As in other studies, no association was found between selenium status and colorectal cancer risk.

\section{References}

1. Combs GF. Selenium. In: Nutrition and Cancer Prevention; Investigating the Role of Micronutrients (Moon TE, Micozzi MS, eds). New York: Marcel Dekker, 1989, pp 389-420.

2. Shamberger RJ, Tytko SA, Willis CE. Antioxidants and cancer: VI. Selenium and age-adjusted human cancer mortality. Arch Environ Health 1976; 31: 231-235.

3. Schrauzer GN, White DA, Schneider CJ. Cancer mortality correlation studies-III: Statistical associations with dietary selenium intakes. Bioinorganic Chemistry 1977: 7: 23-34.

4. Clark L.C, Cantor KP, Allaway WH. Selenium in forage crops and cancer mortality in U.S, counties. Arch Environ Health 1991; 46: 37-42.

5. Levander $\mathrm{OA}$. Considerations on the assessment of selenium status. Fed Proc 1985; 44: 2579-2583.

6. Broghamiei WL McConnell KP, Blotky AI. Relationship between serum selenium levels and patients with carcinoma. Cancer 1976; 37; 1384-1388.

7. Rizk SL, Sky-Peck HII. Comparison between concentrations of trace elements in nornal and neoplastic human breast tissue. Cancer Res 1984; 44: $5390-5394$.

8. Pothier $\mathrm{L}$, Warren WL, Bhargava A, Michielson $\mathrm{C}$, Douglas HO. Plasnina selenium levels in patients with advanced upper gastrointestinal cancer. Cancer 1987; 60: 2251-2260.

9. Willelt WC, Morris JS, Pressel S, et al. Prediagnostic serum selenium and risk of cancer. Lancet 1983; ii: 130-134.

10. Salonen JT, Alfthan G, Huttunen JK, Puska P. Association between serum selenium and the risk of cancer. An J Epidemiol 1984; 120: 342-349.

11. Salonen JT, Salonen R, Lappetelainen R, Maenpaa PH, Alfthan G, Puska P. Risk of cancer in relation to serum concentrations of selenium and vitamins $\mathrm{A}$ and $\mathrm{E}$ : matched case-control analysis of prospective data. Br Med J 1985; 290: 417-420.

12. Peleg I, Morris S, Hames CG. Is serum selenium a risk factor for cancer? Med Oncol Tumor Pharmacother 1985: 2: 157-163.

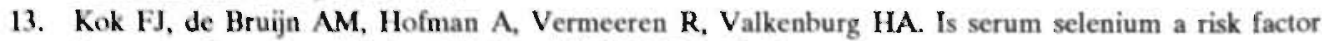
for cancer in men only? Am J Epidemiol 1987; 125: 12-16.

14. Nomura A, Heilbrun LK, Morris JS, Stemmermann GN. Serum selenium and the risk of cancer, by specific sites: case-control analysis of prospective data. J Natl Cancer Inst 1987; 79: 103-108.

15. Virtamo J, Valkeila E, Alfthan G, Punsar S, Huttunen JK, Karvonen MJ. Serum selenium and risk of cancer. A prospective follow-up of nine years. Cancer 1987; 60: 145-148. 
16. Fex G, Pettersson B, Akesson B. Low plasma selenium as a risk factor for cancer death in middleaged men. Nutr Cancer 1987 10: 221-229.

17. Coates RJ, Weiss NS, Daling JR, Morris JS, Labbe RF. Serum levels of selenium and retinol and the subsequent risk of cancer. Am J Epidemiol 1988; 128: 515-523.

18. Ringstad J, Jacobsen BK, Tretli S, Thomassen Y. Serum selenium concentration associated with risk of cancer. J Clin Pathol 1988; 41: 454-457.

19. Knekt P, Aromaa A, Maatela J, et al. Serum selenium and subsequent risk of cancer among Finnish men and women. J Natl Cancer Inst 1990; 82: 864-868.

20. Willett W. Nutritional Epidemiology. New York: Oxford University Press, 1990.

21. Morris JS, Stampfer MJ, Willett W. Dietary selenium in humans; toenails as an indicator. Biol Trace Elem Res 1983; 5: 529-537.

22. Hunter DJ, Morris JS, Chute CG, et al. Predictors of selenium concentration in human toenails. Am J Epidemiol 1990; 132: 114-122.

23. Swanson CA, Longnecker MP, Veillon $\mathrm{C}$, et al. Selenium intake, age, gender, and smoking in relation to indices of selenium status of adults residing in a seleniferous area. Am J Clin Nutr 1990; 52: $858-862$.

24. Van Noord PAH. Selenium and Human Cancer Risk: Nail Keratin as a Tool in Metabolic Epidemiology. Dissertation, Utrecht, Netherlands, 1992.

25. Van den Brandt PA, Goldbohm RA, van 't Veer P, Volovics A, Hermus RJ, Sturmans F, A large-scale prospective cohort study on diet and cancer in The Netherlands. J Clin Epidemiol 1990; 43: 285-295,

26. Van den Brandt PA, Schouten LJ, Goldbohm RA, Dorant E, Hunen PMH. Development of a record linkage protocol for use in the Dutch cancer registry for epidemiological research. Int J Epidemiol 1990; 19: 553-558.

27. Van den Brandt PA, Van 't Veer P, Goldbohm RA, Dorant E, Volovics A, Hermus RJJ, Siurmans F. A prospective cohort study on dietary fat and the risk of postmenopausal breast cancer. Cancer Res (in press).

28. Van den Brandt PA, Goldbohm RA, Van 'i Veer P, Bode P, Hermus RJJ, Sturmans F. Predictors of tocenail selenium levels in men and women. Cancer Epidemiol Biomark Prev (in press).

29. Self SG, Prentice RL. Asymptotic distribution theory and efficiency results for case-cohort studies. Ann Stat 1988; 16: 64-81.

30. Baker RJ. Glim 3.77 Reference Manual. Oxford: Numerical Algorithms Group, 1985.

31. Aitkin M, Anderson D, Francis B, Hinde J. Statistical Modelling in GLIM. Oxford: Oxford University Press, 1989.

32. Knekt P, Aromaa A, Maatela J, et al. Serum vitamin E, serum selenium and the risk of gastrointestinal cancer. Int J Cancer 1988; 42: 846-850.

33. Schober SE, Comstock GW, Helsing KJ, et al. Serologic precursors of cancer. I. Prediagnostic serum nutrients and colon cancer risk. Am J Epidemiol 1987; 126: 1033-1041.

34. Burney PGJ, Comstock GW, Morris JS. Serologic precursors of cancer: serum micronutrients and the subsequent risk of pancreatic cancer. Am J Clin Nutr 1989; 49: 895-900.

35. Halliwell B. How to characterize a biological antioxidant. Free Rad Res Comms 1990; 9: 1-32.

36. Church DF, Pryor WA. Free-radical chemistry of cigarette smoke and its toxicological implications. Environ Health Perspect 1985; 64: 111-126.

37. Nakayama T, Kaneko $M$, Kodama $M$, Nagata $C$. Cigarelte smoke induces DNA single-strand breaks in human cells. Nature $1985 ; 314: 462-464$.

38. Mirvish SS. Effects of vitamins $\mathrm{C}$ and $\mathrm{E}$ on $\mathrm{N}$-nitroso compound formation, carcinogenesis, and cancer. Cancer 1986: 58: 1842-1850.

39. Correa P. A human model of gastric carcinogenesis. Cancer Res 1988; 48: 3554-3560.

40. Groenen PJ, Busink E. Alkylating activity in food products - especially sauerkraut and sour fermented dairy products - after incubation with nitrite under quasi-gastric conditions. Fd Chem Toxic 1988; 26: 215-225. 



\title{
Chapter 17
}

\section{Toenail selenium levels and the subsequent risk of breast cancer*}

Piet A. van den Brandt', R. Alexandra Goldbohm ${ }^{1,2}$, Pieter van 't Veer', Peter Bode', Elisabeth Dorant ${ }^{1}$, Rudolph J.J. Hermus ${ }^{2}$, Ferd Sturmans ${ }^{1}$.

1) Department of Epidemiology, University of Limburg Maastricht, the Netherlands; 2) Department of Nutrition, TNO-Taxicology and Nutrition Institute, Zeist, the Netherlands; 3) Interfaculty Reactor Institute, Delfit University of Technology, Delft, the Netherlands

\begin{abstract}
The assoclation between toenail selenium levels and subsequent breast cancer risk was studled In a prospectlve cohort study on dlet and cancer among 62,573 women aged 55. 69 years. Baseline exposure measurement Included collection of toenall clipplngs, assessment of dietary habits and potential confounders. After 3.3 years of follow-up, 471 microscopically conflimed incident breast cancer cases were detected. Toenall selenium data were available for 355 cases and 1248 members of a randomly selected subcohort of women. The relative rates of breast cancer In Increasing quintiles of toenall selenium were $1.00,0.72,0.62,0.68$ and 0.84 (test for trend $p=0.307$ ), while adjusting for traditional breast cancer risk factors, alcohol and energy Intake. Selenium levels were significantly lower among cases dlagnosed early during follow-up, suggesting an influence of prediagnostic breast cancer. After excluding cases occurring In the first year of follow-up, the relative rates of breast cancer In Increasing quintlles of toenall selenlum were $1.00,0.90,0.76,0.86,0.91$ (trend-p=0.618). It is concluded that selenium status, as measured by toenail selenlum, is not assoclated with breast cancer risk.
\end{abstract}




\section{Introduction}

Studies among rodents have indicated a possible protective effect of selenium on virally or chemically induced mammary tumours. In these studies, both the addition of inorganic selenium (selenite, selenate) and organic selenium (yeast) in the diet have shown a reduction in tumour yield (1-6). In ecological studies among humans, an inverse association between per capita selenium consumption, blood selenium levels or forage crop selenium content and breast cancer mortality has been reported $(7,8)$.

However, case-control studies have yielded inconclusive results. While in some studies serum selenium, a short-term marker of selenium intake, was significantly lower in breast cancer cases compared to controls (9-11), this was not true in another study (12). Selenium in long-term markers, such as erythrocytes $(12,13)$ or toenails (12) was not significantly associated with breast cancer. In prospective cohort studies where prediagnostic selenium status can be measured and thus information bias can be avoided, the majority of studies indicates no association of breast cancer risk with serum or toenail selenium levels (14-20). This latter marker has been suggested as an indicator of long-term selenium status $(21,22)$ and it reflects differences in selenium intake $(23,24)$. We studied the relationship between toenail selenium levels and the risk of breast cancer in a prospective cohort study in the Netherlands, a country with a high incidence of breast cancer (25) and a moderate selenium intake (26).

\section{Methods}

The cohort characteristics and the method of cancer follow-up have been described before $(27,28)$. Briefly, the cohort study started in 1986 when 58,279 Dutch men and 62,573 women aged 55-69 years were enrolled in the cohort. At baseline, cohort members completed a self-administered questionnaire on usual dietary intake and potential confounders and also provided toenail clippings. Following the case-cohort approach for analysis of the data, a subcohort of 3,500 subjects (1688 men, 1812 women) was randomly sampled from the cohort after the baseline exposure measurement. The subcohort has been followed up biennially for vital status information in order to estimate the accumulated persontime in the cohort. Incident cancer cases occurring in the cohort have been identified by record linkage to cancer registries and a pathology register. The analysis in this report is restricted to the cancer incidence in the recently completed $\mathbf{3 . 3}$ year follow-up period from September 1986 to December 1989. The completeness of cancer follow-up was estimated to be $95 \%$ (29). In these 3.3 years of follow-up, a total of 553 breast cancer cases were detected among the cohort of 62,573 women. After excluding incident cases reporting a history of cancer other than skin cancer in the baseline questionnaire $(\tilde{n}=67)$ and cases with in situ carcinoma of the breast ( $n=15), 471$ microscopically confirmed incident breast cancer cases were available for analysis. After excluding prevalent cases with cancer other than skin cancer from the female subcohort of 1812 as well, 1716 subjects remained in this group. Toenail clippings had been provided by 374 breast cancer cases and 1322 female subcohort members. Problems with the detection of toenail selenium (interference by other elements such as calcium) occurred in 7 and 9 of these 374 and 1322 specimens, respectively. Another 12 and 65 specimens were excluded from the breast cancer and subcohort groups, respectively, because the specimens weighed less than $10 \mathrm{mg}$, which would yield unreliable selenium measurements. Thus, toenail selenium data on 355 breast cancer cases and 1248 female subcohort members were available for analysis.

Toenail selenium analyses were carried out by the Interfaculty Reactor Institute (IRI) at Delft University, the Netherlands. Each analytical batch contained toenail 
specimens of cases and subcohort members, and the laboratory personnel conducting the analysis was unaware of case or subcohort status. After cleaning and freeze-drying of the specimens, the selenium content of the toenails was measured by instrumental neutron activation analysis of the metastable-selenium-77 isotope. The specimens were irradiated for 17 seconds in a thermal flux of $1.2 \times 10^{13}$ neutrons. $\mathrm{s}^{-1} \cdot \mathrm{cm}^{-2}$. After a decay time of 20 seconds, gamma radiation of ${ }^{77 \mathrm{~m}} \mathrm{Se}$ was measured for 60 seconds. The accuracy of the method was checked by analysis of a certified Bovine liver standard (Standard Reference Material 1577a of the US National Bureau of Standards). For 26 determinations, a mean value $( \pm$ SD) of $0.70 \pm 0.04 \mu \mathrm{g} / \mathrm{g}$ selenium was observed against a certified value of $0.71 \pm 0.04 \mu \mathrm{g} / \mathrm{g}$. The precision of the method was evaluated by duplicate selenium measurements of specimens from 27 randomly selected subjects; the coefficient of variation was 6.6 percent.

\section{Data analysis}

Mean toenail selenium levels of breast cancer cases and subcohort were compared, as well as the mean levels of case groups, after categorizing the cases according to year of follow-up. In addition to the factors studied in a previously conducted study on predictors of toenail selenium (30), we also evaluated a possible association between toenail selenium level and traditional breast cancer risk factors. This was followed by case-cohort analyses (31), based on the assumption that survival times were exponentially distributed in this follow-up period. For these analyses, toenail selenium levels were categorized into quintiles according to the distribution in the female subcohort. In the age-stratified analyses, we computed Mantel-Haenszel relative rates of breast cancer for each of the quintiles, $95 \%$ confidence intervals, and tests for trend in the relative rates (which were corrected for the additional variance introduced by the subcohort sampling). Similar analyses were conducted for traditional risk factors for breast cancer in the groups of subjects with complete toenail data. In the multivariate case-cohort analyses, relative rates (with corrected $95 \%$ confidence intervals and tests for trend) of breast cancer were computed for each toenail selenium quintile, after adjustment for the effects of traditional breast cancer risk factors and intake of energy and alcohol. To evaluate the potential influence of prediagnostic breast cancer on toenail selenium levels, analyses were also conducted after excluding cases occurring in the first year of follow-up. The analyses were carried out using the GLIM statistical package $(32,33)$. Two-sided p-values are used throughout this report.

\section{Results}

The mean $( \pm S D)$ toenail selenium level in breast cancer cases was $0.569( \pm 0.104)$ $\mu \mathrm{g} / \mathrm{g}$, while in the female subcohort members this value was $0.575( \pm 0.109) \mu \mathrm{g} / \mathrm{g}$ (table 1). When cases were categorized with respect to the year of follow-up in which they were diagnosed, mean toenail selenium levels were increased towards later years of follow-up. Cases in the first and second year of follow-up had significantly lower toenail selenium levels than cases diagnosed in subsequent years (table 1). This suggests a possible modifying influence of prediagnostic breast cancer on toenail selenium level.

Toenail selenium levels were not significantly associated with traditional risk. factors for breast cancer (results not shown), apart from smoking status, which had already been shown to be predictive of toenail selenium levels (29). When an age-stratified analysis of toenail selenium and breast cancer was conducted, the Mantel-Haenszel relative rates were somewhat decreased in the upper four quintiles compared to the lowest quintile, but only the relative rate of 0.64 in the third quintile was significantly different from one. Also, the test for trend was not significant (table 2). 
Table 1. Toenail selenium levels $(\mu \mathrm{g} / \mathrm{g})$ in breast cancer cases according to year of follow-up.

\begin{tabular}{|c|c|c|c|c|}
\hline \multirow[t]{2}{*}{ Group* } & \multirow[t]{2}{*}{ No. of cases } & \multicolumn{3}{|c|}{ Toenail selenium level $(\mu \mathrm{g} / \mathrm{g})$} \\
\hline & & \multicolumn{2}{|l|}{ Mean \pm SD } & \multirow[t]{2}{*}{ p-value } \\
\hline All cases & 355 & $0.569 \pm 0.104$ & & \\
\hline \multicolumn{5}{|c|}{ Year of follow-up } \\
\hline 1 & 89 & $0.558 \pm 0.116$ & & 0.031 \\
\hline 2 & 117 & $0.557 \pm 0.091$ & & 0.022 \\
\hline 3 & 126 & $0.583 \pm 0.108$ & $\neg$ & \multirow{2}{*}{$-\ddagger$} \\
\hline 4 & 23 & $0.593 \pm 0.073$ & $j$ & \\
\hline
\end{tabular}

- Mean ( \pm SD) selenium level in female subcohort members was $0.575( \pm 0.109) \mu g / g(n=1248)$.

$\uparrow$ T-test between strata, based on In-transformed toenail selenium levels.

$\mp$ Reference category.

Table 2. Relative rate of breast cancer according to toenail selenium level in stratified and multivariate analyses.

Type of adjustment

Quintile of toenail selenium level (boundaries in $\mu \mathrm{g} / \mathrm{g}$ )

Test. for trend

$\begin{array}{lllllll}1 \cdot & 2 & 3 & 4 & 5 & x^{2} & \text { (p-value) }\end{array}$

Age-ștrailified

\begin{tabular}{|c|c|c|c|c|c|c|c|}
\hline $\begin{array}{l}\text { No. of cases } \\
\text { (Person years } \\
\text { in subcohort) }\end{array}$ & $\begin{array}{l}87 \\
(811)\end{array}$ & $\begin{array}{l}66 \\
(813)\end{array}$ & $\begin{array}{l}57 \\
(816)\end{array}$ & $\begin{array}{l}66 \\
(813)\end{array}$ & $\begin{array}{l}79 \\
(804)\end{array}$ & & \\
\hline $\begin{array}{l}\mathbf{R R}_{\mathbf{M H}} \\
(95 \% \text { CI })\end{array}$ & 1.00 & $\begin{array}{l}0.76 \\
(0.53-1.09)\end{array}$ & $\begin{array}{l}0.64 \\
(0.44-0.94)\end{array}$ & $\begin{array}{l}0.77 \\
(0.53-1.10)\end{array}$ & $\begin{array}{l}0.93 \\
(0.65-1.33)\end{array}$ & 0.198 & $(0.656)$ \\
\hline
\end{tabular}

Multivariate modelt

\begin{tabular}{|c|c|c|c|c|c|c|c|}
\hline $\begin{array}{l}\text { No. of cases } \\
\text { (Person years } \\
\text { in subcohort) }\end{array}$ & $\begin{array}{l}67 \\
(631)\end{array}$ & $\begin{array}{l}48 \\
(628)\end{array}$ & $\begin{array}{l}46 \\
(670)\end{array}$ & $\begin{array}{l}49 \\
(643)\end{array}$ & $\begin{array}{l}60 \\
(634)\end{array}$ & & \\
\hline $\begin{array}{l}\text { RR } \\
(95 \% \mathrm{CI})\end{array}$ & 1.00 & $\begin{array}{l}0.72 \\
(0.47-1.11)\end{array}$ & $\begin{array}{l}0.62 \\
(0.40-0.96)\end{array}$ & $\begin{array}{l}0.68 \\
(0.44-1.05)\end{array}$ & $\begin{array}{l}0.84 \\
(0.55-1.27)\end{array}$ & 1.044 & $(0.307)$ \\
\hline
\end{tabular}

Fxcluding cases from first year of follow-upt

\begin{tabular}{|c|c|c|c|c|c|c|c|}
\hline $\begin{array}{l}\text { No, of cases } \\
\text { (Person years } \\
\text { in subcohort) }\end{array}$ & $\begin{array}{l}44 \\
(437)\end{array}$ & $\begin{array}{l}39 \\
(436)\end{array}$ & $\begin{array}{l}37 \\
(465)\end{array}$ & $\begin{array}{l}40 \\
(444)\end{array}$ & $\begin{array}{l}42 \\
(438)\end{array}$ & & \\
\hline $\begin{array}{l}\text { RR } \\
(95 \% \mathrm{CI})\end{array}$ & 1.00 & $\begin{array}{l}0.90 \\
(0.55-1.46)\end{array}$ & $\begin{array}{l}0.76 \\
(0.47 \cdot 1.23)\end{array}$ & $\begin{array}{l}0.86 \\
(0.53-1.39)\end{array}$ & $\begin{array}{l}0.91 \\
(0.56-1.46)\end{array}$ & 0.248 & $(0.618)$ \\
\hline
\end{tabular}

- Reference category.

† The model included terms for age, history of benign breast disease, maternal breast cancer, breast cancer in sister(s), age at menarche, age at menopause, oral contraceptive use, parity, age at first birth, Quetclet index, education, current cigarette smoking, intake of alcohol and energy. 
In the group of subjects with complete toenail data, the effects of the established breast cancer risk factors were in the anticipated direction. After stratification by age, an elevated risk was observed for those with a history of benign breast disease (MantelHaenszel relative rate, $\mathrm{RR}=1.87 ; 95 \%$ confidence interval, $\mathrm{CI}, 1.27$ to 2.74 ), history of breast cancer in mother $(\mathrm{RR}=1.68 ; 95 \% \mathrm{CT}, 0.99$ to 2.86$)$ and history of breast cancer among one or more sisters $(\mathrm{RR}=1.76 ; 95 \% \mathrm{CI}, 1.12$ to 2.77$)$. Parity showed a significantly inverse association with breast cancer risk (chi for trend $=-2.58 ; p=0.010$ ). Age at first birth was significantly positively associated with the risk of breast cancer (chi for trend $=2.11 ; p=0.035$, among parous only). Age at menarche was inversely associated with breast cancer risk, although not significantly (chi for trend=-1.32; $\mathrm{p}=0.187$ ), whereas age at menopause showed a borderline significant positive association (chi for trend $=1.90 ; p=0.057$ ). No significant associations were observed with menopause induced by hormones or surgically ( $R R=0.96 ; 95 \% \mathrm{CI}, 0.69$ to 1.32 ), use of oral contraceptives ( $R R=1.02 ; 95 \% \mathrm{Cl}, 0.76$ to 1.37 ), level of education (chi for trend $=0.365 ; p=0.715)$, current cigarette smoking $(R R=0.98 ; 95 \% \mathbf{C l}, 0.73$ to 1.32$)$ or Quetelet index (chi for trend $=-0.68 ; \mathrm{p}=0.497$ ).

When the association between toenail selenium and breast cancer risk was tested in a multivariate model containing terms for the mentioned established and potential breast cancer risk factors, energy intake and alcohol use, the relative rates for increasing quintiles of toenail selenium were estimated at $1.00,0.72,0.62,0.68$ and 0.84 , respectively (table 2). Again, the relative rate in the third quintile was significantly below the null value, but the trend was not significant (trend-p $=0.307$ ). When a similar model was fitted after excluding cases diagnosed in the first year of follow-up, the relative rates in the upper four quintiles were closer to the null value and the relative rate in the third quintile no longer significant (table 2). Additional exclusion of cases occurring in the second year of follow-up again yielded no association between selenium and breast cancer (trend- $\mathrm{p}=0.577$ ).

\section{Discussion}

We did not find a significant inverse association between toenail selenium level and breast cancer risk in this prospective study among postmenopausal women, after excluding cases occurring in the first year of follow-up. Our findings on lowered toenail selenium levels in cases occurring early in the follow-up period are in contrast with those of Hunter et al. (20), who reported no influence of prediagnostic breast cancer on toenail selenium levels. However, in another cohort study, prevalent breast cancer cases showed slightly lower toenail selenium concentrations than incident cases (19). The possible influence of prediagnostic breast cancer on lowering selenium status is also in accordance with observations on normal and tumour tissue of breast cancer patients, indicating a sequestration of selenium by the tumour tissue (34). Furthermore, it has been found in case-control studies that plasma and erythrocyte selenium levels were lowered only in cases with large tumours (12) and that selenium levels were inversely associated with breast cancer stage (10). Given the possible modulation of selenium status by prediagnostic breast cancer, the results of case-control studies are difficult to interpret. Case-control studies on serum selenium showed varying, results (9-12); a possible explanation for this might be a dissimilarity in the stage distribution of breast cancer patients between the various studies. Case-control studies that have measured erythrocyte selenium showed no association with breast cancer $(12,13)$. Toenail selenium was modestly inversely related to breast cancer in a recent case-control study (12). Indeed, if we would limit our analysis to the first year of follow-up only, the 
relative rate estimate for increasing quintiles of toenail selenium would be $1.00,0.37$, $0.39,0.43,0.73$, with the middle three estimates being significantly different from unity.

Two prospective studies on toenail selenium and breast cancer have been published, both using a nested case-control analysis. Hunter et al. (20) found no association between toenail selenium and breast cancer in their study, encompassing 434 incident breast cancer cases originating from a cohort of 62,641 US nurses after a follow-up of 4.4 years; there was also no association when pre- and postmenopausal women were analyzed separately. The study by Van Noord et al. (19) among 8760 premenopausal women ( 27 incident cases after two years of follow-up) also showed no association with selenium levels. Apart from these toenail studies, a number of prospectively nested case-control studies using serum selenium have been conducted, from which specific results on breast cancer risk have been reported (14-18). The follow-up periods covered in these studies ranged from five $(14)$ to over 10 years $(15,18)$. None of these serum studies showed a significant association between serum selenium and breast cancer risk, but it should be mentioned that the number of breast cancer cases in most of these studies was too small to perform statistically meaningful analyses specifically for this site (14-17).

In conclusion, we found no evidence for an inverse association between selenium status, as measured by toenail selenium levels, and the risk of breast cancer. The relatively low toenail levels observed among cases occurring carly during follow-up illustrate the need to interpret case-control studies on selenium and breast cancer with caution.

\section{References}

1. Schrauzer GN, ishmael D. Effects of selenium and of arsenic on the genesis of spontaneous mammary tumors in inbred $\mathrm{C}_{3} \mathrm{H}$ mice. Ann Clin Lab Sci 1974; 4: 441-447.

2. Thempson III, Becci PJ. Selenium inhibition of $\mathbf{N}$-methyl-N-nitrosurea-induced mammary carcinogenesis in the rat. J Natl Cancer Inst 1980; 65: 1299-1301.

3. Schrauzer GN, MeGinness JE, Kuehn K. Effects of temporary selenium supplementation on the genesis of spontaneous mammary tumors in inbred female $\mathrm{C}_{3} \mathrm{H} / \mathrm{St}$ mice. Carcinogenesis 1980; 1 : 199-201.

4. Welsch CW, Goodrich-Smith M: Brown CK, Greene HD, Hamel EJ. Selenium and the genesis of murine mammary tumors. Carcinogenesis 1981; 2: 519-522.

5. Ip C. Prophylaxis of mammary neoplasia by selenium supplementation in the initiation and promotion phases of chemical carcinogenesis. Cancer Res, 1981; 41: 4386-4390.

6. Medina D, Shepherd F. Selenium-mediated inhibition of 7,12-dimethyl-benz(a)anthracene-induced mouse mammary tumorigenesis. Carcinogenesis 1981; 2: 451-455.

7. Schrauzer GN, White DA, Schneider CJ. Cancer mortality correlation studies. III: Statistical associations with dietary selenium intakes. Bioinorg Chem 1977; 7: 23-34.

8. Clark LC, Cantor KP, Allaway WH. Selenium in forage crops and cancer mortality in U.S. counties. Arch Environ Health 1991; 46: 37-42.

9. McConnell KR, Jager RM, Bland KI, Blotcky AJ. The relationship of dietary selenium and breast cancer. J Surg Oncol 1980; 15: 67-70.

10. Chaitchik S, Shenberg C, Nir-El Y, Mantel M. The distribution of selenium in human blood samples of Israeli population - comparison between normal and breast cancer cases. Biol Trace Elem Res 1988; 15: 205-212.

11. Schrauzer GN, Molenaar T, Mead $S_{n}$ Kuehn K, Yamamoto H, Araki E. Selenium in the blood of Japanese and American women with and without breast cancer and fibrocystic disease. Jpn J Cancer Res 1985; 76: 374-377.

12. Van 'I Veer P, Van der Wielen RPJ, Kok FJ, Hermus RJJ, Sturmans F. Selenium in diet, blood, and toenails in relation to breast cancer: a case-control study. Am J Epidemiol 1990; 131: 987-994.

13. Meyer F, Verrault R. Erythrocyte selenium and breast cancer risk. Am J Epidemiol 1987; 125: 917 919.

14. Willett WC, Morris JS, Pressel S, Taylor JO, Polk BF, Stampfer MJ, et al. Prediagnostic serum selenium and risk of cancer. Lancet 1983; ii: $130-134$. 
15. Peleg I, Morris S, Hames CG. Is serum selenium a risk factor for cancer? Med Oncol Tumor Pharmacother 1985; 2: 157-163.

16. Coates RJ, Weiss NS, Daling JR, Morris JS, Labbe RF. Serum levels of selenium and retinol and the subsequent risk of cancer. Am I Epidemiol 1988; 128: 515-523.

17. Ringstad J, Jacobsen BK, Tretli S, Thomassen Y. Serum selenium concentration associated with risk of cancer. J Clin Pathol 1988; 41: 454-457.

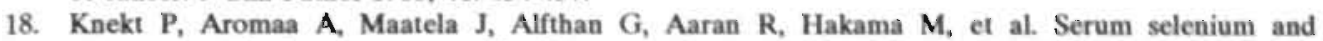
subsequent risk of cancer among Finnish men and women. J Natl Cancer Inst 1990; 82: 864-868.

19. Van Noord PAH, Collette HJA, Maas MJ, de Waard F. Selenium levels in nails of premenopausal breast cancer patients assessed prediagnostically in a cohort-nested case-referent study among women screened in the DOM project. Int J Epidemiol 1987; 16(SuppL): 318-322.

20. Hunter DJ, Morris JS, Stampfer MJ, Colditz GA, Speizer FE, Willett WC. A prospective study of selenium status and breast cancer risk. JAMA 1990; 264: 1128-1131.

21. Willett W. Nutritional Epidemiology. New York: Oxford. University Press, 1990.

22. Morris JS, Stampfer MJ, Willett WC. Dietary selenium in humans. Toenails as an indicator. Biol Trace Elem Res 1983; 5: 529-537.

23. Hunter DJ, Morris JS, Chute CG, Kushner E, Colditz GA, Stampfer MJ, et al. Predictors of selenium concentration in human toenails. Am J Epidemiol 1990; 132: 114-122.

24. Swanson CA, Longnecker MP, Veillon C, Howe SM, Levander OA, Taylor PR, et al. Selenium intake, age, gender, and smoking in relation to indices of selenium status of adults residing in a seleniferous area. Am J Clin Nutr 1990; 52: 858-862.

25. Muir C, Waterhouse J, Mack T, Powell J, Whelan S, eds. Cancer incidence in five continents. Vol V. IARC Sci Publ no. 88. Lyon: International Agency for Research on Cancer, 1987.

26. Van Dokkum W, De Vos RH, Muys TH, Wesstra JA. Minerals and trace elements in total diets in the Netherlands. Br J Nutr 1989; 61: 7-15.

27. Van den Brandt PA, Goldbohm RA, van 't Veer P, Volovics A, Hermus RJ, Sturmans F. A large-scale prospective cohort study on diet and cancer in The Netherlands. J Clin Epidemiol 1990; 43: $285-295$,

28. Van den Brandt PA, Schouten LJ, Goldbohm RA, Dorant E, Hunen PMIH. Development of a record linkage protocol for use in the Dutch cancer registry for epidemiological research. In1 J Epidemiol 1990; 19: 553-558.

29. Van den Brandt PA, Van 'I Veer P, Goldbohm RA, Dorant E, Volovics A, Hermus RJJ, Sturmans F. A prospective cohort study on dietary fat and the risk of postmenopausal breast cancer. Cancer Res (in press).

30. Van den Brandt PA, Groldbohm RA, Van 't Veer P, Bode P, Hermus RJJ, Sturmans F. Predictors of toenail selenium levels in men and women. Cancer Epidemiol Biomark Prev (in press).

31. Self SG, Prentice RL. Asymptotic distribution theory and efficiency results for case-cohori studies. Ann Stat 1988; 16: 64-81.

32. Baker RJ. Glim 3.77 Reference Manual. Oxford: Numerical Algorithms Group, 1985.

33. Aitkin M, Anderson D, Francis B, Hinde J. Statistical Modelling in GLIM. Oxford: Oxford University Press, 1989.

34. Rizk SL, Sky-Peck HH. Comparison between concentrations of trace elements in normal and neoplastic human breast tissue. Cancer Res 1984; 44: 5390-5394. 



\section{Chapter 18}

\section{Epilogue}

Instead of elaborating on all results described in the various chapters, the primary objective of this epilogue is to evaluate the choice to conduct a prospective cohort study rather than conducting several case-control studies. In addition, we will discuss the results on selenium and cancer now that the analyses of the relation between selenium status and cancer sites of initial interest (stomach, colon, rectum, breast and lung) have been completed.

\section{Choice of study design}

Textbooks on epidemiology state that prospective cohort (follow-up) studies may provide a better basis for inference than other types of epidemiologic research, except experiments (1). The reason for this is that case-control studies may suffer from selection and information or recall bias, which are avoided in a prospective design. Potential drawbacks of prospective studies - most of them related to the large scale required for such studies - include recruitment of a cohort of sufficient size, follow-up of that cohor for the end-points of interest, the possibly less accurate assessment of exposure, and last but not least, the presumably high costs. Did the advanitages outweigh the disadvantages in the present prospective study as compared to a case-control study? We will attempt to formulate a (preliminary) answer by reviewing the points successively.

\section{Selection bias}

Selection bias may operate in case-control studies if enrollment of cases or controls is associated with exposure, either direct or mediated by some other factor. Known as well as unknown factors may introduce this type of bias. If these factors are known, selection bias can be controlled by proper selection of case and control groups $(2,3)$. In prospective studies selection bias does not play a role, since cases originate from the cohort. Substantial non-response of either cases or controls in case-control studies may also result in selection bias, since response may be related to the exposure of interest, but differentially so for cases and controls. For example, in a recent case-control study on oral contraceptives and breast cancer the non-responders among the control subjects appeared to have used less oral contraceptives than the responders, resulting in a lower relative risk estimate (4). In prospective cohort studies, bias due to non-response is presumed to be absent, since there are no cases yet at the time of the baseline exposure measurement. Thus, although non-response may introduce selection that is related to exposure, this is likely to be independent of the (future) case status of the cohort members and will, therefore, not introduce bias. In cohort studies, however, the counterpart of selection bias due to non-response is exposure-related loss to follow-up; this will be discussed later.

\section{Information bias}

Information bias is considered to be a more serious problem than selection bias in some case-control studies, in particular in studies that assess dietary habits. Information bias can arise as a result of altered dietary habits in cases due to the disease under study (e.g., cancers of the digestive tract). Even if the participant is asked to recall his habits before the development of symptoms, recall bias may play a role since studies have consistently shown that recall from the past is influenced by current habits $(5,6,7)$. 
Studies on diseases that do not directly affect dietary habits (e.g., breast cancer) may also suffer from recall bias, since cases may be more aware of their dietary habits and more motivated to recall them. Although empirical evidence for the existence of information bias in case-control studies on diet and cancer is limited, the plausibility of its existence has been one of the main reasons to launch prospective cohort studies (8). Available evidence comes from two cohort studies. In the Nurses' Health Study, 398 breast cancer cases, who had completed a baseline questionnaire, again completed the questionnaire after diagnosis of the disease. The relative risk for the highest versus the lowest quintile of fat consumption was 0.97 in the prospective analysis compared to 1.43 in the case-control analysis (9). A similar study did not provide evidence for recall bias in a case-controll study on breast cancer (10).

Prospective cohort studies, however, may also suffer from information bias, albeit only bias due to a change in dietary habits caused by symptoms of prediagnostic disease. In our data there was some evidence for this type of bias. For example, alcohol and energy intake in men with colorectal cancer as well as toenail selenium in subjects with breast and colon cancer appeared to be lower in cases diagnosed in the first year of follow-up. However, this bias can be corrected by excluding cases with a short period between baseline exposure measurement and diagnosis as we have done.

\section{Cholce of the study population and recruitment of the cohort}

One of the attractive options in cohort studies compared to case-control studies is that the investigator can choose the study population. Of course, the choice may also depend on practical aspects of cohort recruitment and follow-up. Given the existing opportunities in the Netherlands, we were able to assemble a cohort originating from the general population. Several well-known cohort studies from other countries have made use of professional groups because sampling from the general population was not feasible (e.g., Nurses' Health Study (11) or Health Professionals' Follow-up Study (12)). Studying such groups, particularly those occupied in health-related fields, may have several advantages as compared to a general population cohort. Besides the availability of professional listings for recruitment, and the ability of these subjects to complete detailed questionnaires, a very important point is the access to diagnostic information that the investigators can obtain through cohort members (who can be both patients and treating physicians). This enables the investigators to study a range of different diseases (e.g., cancer, heart disease, osteoporosis, gallstones, hypertension (12-15)). A final point is the lack of confounding by occupation which is imposed by the restriction to the occupational group in the design.

However, studying the general population has several important advantages as well which, conversely, can be viewed as disadvantages of studying an occupational group. First, the results may be more easily extrapolated to the population for which all the research is meant. This may be important when translating the results into cancer prevention programs for the general public and when use is made of population attributable risks. Second, by studying the general population, one may provide a greater contrast in the exposures of interest since diet is related to socioeconomic status. Furthermore, it enables the investigator to study interaction between diet and, for example, occupation in relation to cancer. Surprisingly, the fields of occupational cancer research (i.e., dealing with occupation as possible cause) and dietary cancer research are almost totally separated, while it is not unlikely that these two exposures may modify each others' effects on cancer risk. By studying our general population cohort, we were also able to assess effects of dietary exposures on cancer in men and women simultaneously (e.g., selenium and various cancers, alcohol and colorectal cancer). When cohort studies are conducted in certain professional groups these are often restricted to one gender. Subsequent comparisons of results from separate male 
and female cohort studies may be more difficult to interpret, because different results may be attributable to variations in design, exposure measurement techniques, quality of follow-up information, and so on.

A third option is to recruit a cohort from an ongoing screening program (e.g., 16,17). With regard to pros and cons this can be viewed as a mixture of the two previous options. The screening program is usually offered to the general population, but by the very nature of the screening it is often limited to a particular gender. A distinct advantage of this approach is that biomarkers of exposure or susceptibility can be obtained during the screening visit(s). These personal contacts also facilitate followup of the cohort members. However, because screening programs are gradually introduced into the population, more time is needed to assemble the totall cohort. In the first years of a study this may be a disadvantage since it takes longer until a sufficient number of cases has emerged. Also, the staggered entry and inclusion of expensive biomarker measurements often results in somewhat smaller cohorts.

Some cohort studies bave created a greater contrast in dietary exposure by including vegetarians (18). We have also made an attempt to overrepresent vegetarians in the cohort. Since the population registries do not contain such data, we had to call for participation in magazines, and in leaflets displayed in health food stores and packaged in vegetarian products. Although vegetarians could potentially be overrepresented by a factor five at most, as was deducted from the observation that $20 \%$ of the vegetarians who applied for participation were also present in the random sample from the general population, the specially recruited vegetarians increased the proportion of vegetarians in the cohort from 1.0 to $1.2 \%$ only. Nevertheless, this implies that in a cohort of 120,000 people a substantial number is vegetarian; after a longer follow-up period it will be interesting to study cancer incidence in this particular group. The unsuccessful attempt to overrepresent vegetarians does illustrate, however, how inefficient such recruitment procedures are: (a) response to the call for participation was extremely low in comparison with the population sampled from the population registries, who received a personal invitation letter; (b) although the calls for participation included criteria, such as age and residence, one third of the applicants did not meet the criteria; (c) the names, birth dates and addresses provided by the applicants were often incomplete, illegible or incorrect, resulting in decreased sensitivity of record linkage with the cancer registries. Thus, the yield of this recruitment procedure (larger contrast in dietary habits) relative to the amount of work involved was very low. In contrast, sampling of the general population from the population registries appeared to be extremely efficient, even when we take into account the relatively high costs of sampling and the rather low response $(36 \%)$. The advantages, i.e. selection of the required age group, complete and correct identifying information not requiring computer entry, outweighed the disadvantages (costs) by far. In 1985, it appeared to be relatively easy to get permission from the municipalities to draw a large sample, since only $7 \%$ of the municipalities refused. Since then, however, privacy regulations have become more riged, resulting in less efficient procedures or even the impossibility of sampling. For epidemiologic research this is a very unfortunate development, which can only be diverted when researchers and administrators would agree on guidelines for handling privacy-sensitive (identifying) information.

\section{Follow-up}

Completeness of follow-up is important in prospective studies for two reasons: (1) loss to follow-up may introduce (positive or negative) bias into the results, if this loss is related to the exposure of interest and (2) loss to follow-up that is random, i.e. not dependent on the exposure of interest leads to loss of efficiency (power), since less 
cases with the disease of interest are identified. In our cohort study, loss to follow-up appeared to be very small as a result of a high degree of coverage by the cancer registries and PALGA and high accuracy of the record linkage. We therefore conclude that bias or decreased efficiency due to loss of follow-up is no issue in this study.

The follow-up procedures appeared to be very feasible, although time had to be invested in the development of record linkage procedures. It appeared that in a relatively short time period, the cancer registries have successfully organized the data collection and in such a way that epidemiological studies like this cohort study are greatly facilitated. In this respect, the patient-based cancer registry data require less work for the investigators than the specimen-based PALGA data. In the PALGA data base multiple pathology reports of one patient are stored as separate records without a patient identification key, requiring extensive proceduresi using additional identifying information to assign records belonging to a specific patient. The diagnostic information of the aggregated records then has to be coded per tumor site into ICD-Oncology. The efficiency of this procedure could be improved substantially if pathology laboratories would label records as belonging to a particular patient. Nevertheless, the PALGA data were of great importance for us, given our interest is in microscopically confirmed cancers, because the cancer registries were not yet fully covering the whole country at the start of the study. An additional advantage of PALGA is that the data are available shortly after diagnosis.

The possibilities for follow-up through cancer registries and PALGA contrast sharply with follow-up for causes of death. Although privacy considerations are likely to be even more important for incident cancer data than for mortality data, privacy regulations have been formulated such that they do not hamper use of the cancer registries and PALGA for prospective studies. Unfortunately, record linkage of cohorts to the national data base of causes of death, maintained by the Central Bureau of Statistics, is hindered by privacy arguments (19).

\section{Assessment of exposure}

Large-scale cohort studies have to assess exposure using relatively inexpensive methods that do not require involvement of personnel such as interviewers. For dietary assessment a self-administered food frequency questionnaire is then the method of choice. Compared to case-control studies, in which a more elaborate, intervieweradministered method of diet assessment can be used, the food frequency questionnaire may be less accurate. Although many data are available on the validity of food frequency questionnaires, such data are scarce for the more elaborate methods. Pietinen et al. (20) used an extensive, interviewer-checked, guestionnaire with picture booklets. Its validity, as compared to a diet record method, however, is similar to the food frequency questionnaire (FFQ) used in our study. Van Beresteijn et al. (21) used a very comprehensive diet history method. Its reproducibility, which may give an indication of the validity, is substantially higher than that of our FFQ. We may conclude that a FFO is less accurate than an extensive, interviewer-administered method. The consequence is that the observed associations between diet and cancer are somewhat more attenuated, requiring larger numbers of cases $(22,23)$. For example, Walker and Blettner (20) have calculated that in a cohort study $50 \%$ more cancer cases. are required to account for an assumed 0.10 decrease in correlation between the FFQ and "true" intake as compared to the correlation between an elaborate method and "true" intake. Although it is possible to correct relative risk estimates that are attenuated by imperfect measurement methods, it is important in this respect that the estimates should not biased by selection and information bias (23).

An advantage of case-control studies is that biomarkers of exposure (given that they represent long-term status and are not influenced by disease) can sometimes be 
included in the exposure assessment, whereas this is momentarily often too expensive for large-scale cohort studies. An exception to this rule may be provided by cohorts recruited from screening programs, for which exposure and biomarker assessment can be combined with the screening. We also considered the possibility of including biomarkers of exposure in our cohort study. In a pilot study we studied the feasibility of including the following biochemical parameters: fecal $\mathrm{pH}$, nitrite concentration of saliva and selenium concentration in toenails. The pilot study showed that assessment of fecal $\mathrm{pH}$ and salivary nitrite by the participants (using test strips) led to considerable misclassification when comparing it with the laboratory analyses of the returned stools and saliva specimens, respectively $(24,25)$. Only toenaill specimens appeared to be feasible to collect on a large scale. This study has shown the potential usefulness of toenails in epidemiologic research. There is clearly a need for developing more exposure biomarkers that reflect the long-term status of particular nutrients or metabolites and have an established feasibility in (large-scale) epidemiologic studies. Likewise, there is a need for studying feasibility aspects, sources of variation and, in particular, the predictive value of disease biomarkers $(26,27)$.

\section{Conclusion}

Considering the points discussed above, we think that this cohort study has already outweighed a case-control study on diet and cancer as far as the methodological aspects are concerned. Furthermore, the conduct of the study appeared to be very feasible. With respect to the costs, we have calculated that for the first five years of follow-up the cost per case of breast, stomach, colorectal and lung cancer amounted to approximately NLG 1000. Prolongation of follow-up of the cohort will further reduce the cost invested per identified case. We had to devote much time to development and validation of methods, e.g., the dietary questionnaire and its further processing and the record linkage to the cancer registries and PALGA. It is to be expected that future epidemiologic (cohort) studies may benefit from this work.

\section{Selenium and cancer}

Following observations on a possible protective effect of selenium in animal studies and ecological studies among humans, a large number of prospective cohort studies have been conducted in the 1980s on the hypothesized inverse association between serum selenium and cancer risk. Although the cohorts studied were considered large, the actual number of incident cancer cases was rather small. Therefore, in virtually all of these cohort studies analyses focused on selenium and overall cancer risk. The sitespecific number of cases was usually too small to perform statistically meaningful analyses, with the exception of the Finnish cohort study by Knekt et al. (28) and the Washington County cohort (29). The informativeness of relationships with overall cancer risk is limited, since it is unlikely that a single (nutritional) factor would be protective against all or most types of cancer. Indeed, the etiology of most cancers seems to be so variable that it would be very fortunate if a ubiquitous cancer-preventive agent would exist. Differences in results between the conducted cohort studies on selenium and cancer risk may therefore be partially explained by differences in distribution of the tumor site. The sample size of serum selenium studies is usually limited, because of the invasive sampling and logistic problems (collection, transport, storage). Collecting toenail clippings is more attractive because sampling is noninvasive, collection can be done by study subjects themselves, transport by mail poses no problem and specimens can be stored at room temperature. Furthermore, they reflect long-term selenium status and differences in selenium intake (8). A recent study among Finnish 
men also showed that toenails reflect selenium intake from diets rich in organic selenium compounds (the predominant form in the human diet) but not from inorganic selenium compounds such as selenite or selenate (30). The collection of toenails hence enables investigators to assemble a larger cohort and consequently perform site-specific analyses. Indeed, two prospective studies on breast cancer and toenail selenium have been published previously $(17,31)$.

When studying the association between selenium and cancer, this should preferably be done in a setting in which sufficient variation in selenium levels exists between individuals and where selenium intake may be limiting. In this respect, it has been noted (32-34) that discrepancies in the findings from the cohort studies may partially be explained by differences in the range of selenium levels that has been investigated in the various countries. The lowest mean serum selenium levels have been reported from Finland and New Zealand, whereas in North Americans the levels are two or three times higher (35). Indeed, most of the studies that show a negative association originate from Finiand where selenium intake used to be low (28). Selenium levels in the Finnish population have recently increased, following the use of selenium-enriched fertilizer to increase the selenium content of the soil, and consequently of foods (36). Studying the relationship between selenium and cancer was also interesting in the Netherlands, because the estimated intake is moderate and serum selenium levels are intermediate between those reported from New Zealand and those in the USA (37), as we found for toenail selenium levels (38).

The postulated mechanisms by which selenium may exert a beneficial effect include altered metabolism of carcinogens, reduction of the mutagenicity of carcinogens, inhibition of cell proliferation, stimnulation of the immune system and protection against oxidative damage via the selenoenzyme glutathione peroxidase $(34,39,40)$. The latter mechanism has received most attention. If selenium indeed would be protective against oxidative stress, studying smoking-related cancers might be informative since smoking induces oxidative stress $(41,42)$. Now that we have studied the association between toenail selenium and the risk of several cancers simultaneously after 3.3 years of followup and can compare these results, such a pattern indeed seems to emerge: the inverse association with selenium is present in cancers of the lung and stomach, where smoking plays a more important role in the etiology, whereas it is not seen in the other cancer sites studied, i.e. breast, colon, rectum.

This pattern is in line with site-specific results from other studies. Knekt et al. (28) observed the strongest association with serum selenium for lung, stomach and pancreatic cancer in Finland, while in the Washington County cohort significant inverse associations were found for pancreatic and bladder cancer but not for lung cancer (29). In the majority of the other, smaller, cohort studies a nonsignificant inverse association with lung cancer was seen which was often stronger than for total cancer. Finally, the risk of oral cancer, another smoking-related cancer, was inversely related to toenail selenium levels in a recent case-control study; this association was further modified by the level of vitamin $C$ intake (43). We also found evidence of a modification of the effect of selenium by the antioxidant vitamins $C$ and $B$-carotene, which lends additional support to the antioxidant hypothesis regarding selenium and cancer. We could not evaluate possible effect modification by vitamin $E$, since data on vitamin $E$ content are currently not available in the Dutch NEVO food table. This is unfortunate, since an interaction between this antioxidant vitamin and selenium has been described in several studies regarding overall cancer risk $(28,44-46)$. Extending the food table to vitamin $E$, other antioxidants and (yet) nonnutritive elements is warranted, as is further study of the interactions between antioxidants such as vitamin $C$, vitamin $E$ and selenium with regard to cancer risk. 
Of course, a disadvantage of our study is that no definitive conclusion can be drawn about a true independent inverse association of toenail selenium with lung and stomach cancer. Although we have controlled for smoking in our analyses fairly rigorously using pack-years of current and past smokers, residual confounding by smoking cannot be excluded, given the observation that smoking is inversely related to toenail and other selenium status parameters $(8,38,47)$. Therefore, it may be that a low toenail selenium level is only a consequence of smoking and that it has no independent contribution to the occurrence of these tumors. We did find an inverse association between toenail selenium and lung cancer within categories of smoking (never/ex/current), but it is then still possible that heavy smokers have both the lowest selenium levels and the highest cancer risk. A randomized controlled trial would be needed to solve this issue more definitively; several trials have been considered (34,48-51).

Finally, a criticism of our selenium analyses may be the relatively short follow-up time of 3.3 years. This potential problem was addressed by evaluating mean toenail selenium levels of cases occurring in each of the years of follow-up, as well as by excluding cases diagnosed in the first year of follow-up from the relative risk analyses. Only for breast and colon cancer toenail selenium levels appeared to be decreased by prediagnostic cancer, which nnay be due to lowered selenium intake, altered absorption and excretion or sequestration by the tumors $(33,52,53)$. Because we did not find an effect of prediagnostic stomach cancer on toenail selenium levels, the first possibility seems less likely for breast and colon cancer. Nevertheless, the lowered selenium levels of breast and colon cancer cases occurring early during follow-up indicate the need to interpret case-control studies on this issue with caution. The number of stomach cancer cases after 3.3 years of follow-up was still rather low for analyzing the association with toenail selenium. For this site, as well as for other, less frequent sites a longer follow-up period is warranted to study the relationship with selenium status in a more definitive way.

Also, when more cases are available, it will be possible to evaluate more fully effect modification by other antioxidants. The size of most epidemiological studies precludes an investigation of interaction between several exposures. The size of our cohort is such that, with a sufficiently long follow-up, effect modification can be studied with regard to the occurrence of cancer. What becomes more important then is information on the reproducibility of toenail selenium levels over time (31). Included in our dietary reproducibility study was a collection of toenail clippings. Due to a lack of funds we have not yet been able to assess the reproducibility using these five repeated measurements. Information on intraindividual variation (of exposure and covariates) can be used to correct relative rates for attenuation. This requires further development and use of statistical methods that take into account random errors in both exposure and covariates $(54,55)$. Irrespective of whether selenium will be shown to be protective against (smoking-related) cancers such as lung cancer, it is important to note that the magnitude of the smoking-lung cancer association is much stronger than that between selenium and lung cancer. Cancer prevention through smoking reduction therefore remains substantially more important.

\section{Concluding remarks}

Many epidemiologists and textbooks commonly recommend prospective investigators to use a prospective study design to avoid several biases and to conduct pilot studies to develop the methods of data collection. Fewer recommend their colleagues also to document the validity and reproducibility of these methods, as applied in the actual epidemiological study. This latter point is clearly appreciated by 
methodological investigators but much less so by funding agencies. Furthermore, the general rule of funding research projects for four years is hardly compatible with conducting a truly prospective study with sufficient follow-up time. This then usually leads to carrying out a case-control study or may lead to a prospective study with an unrealistically short follow-up with low power and, depending on type of exposure, interpretation problems similar to case-control studies.

We have conducted a prospective cohort study on diet and cancer that would both yield a number of answers to diet-cancer questions in a relatively short period of time and satisfy methodological interests. Conducting such a study in four years is impossible, certainly after incorporating a number of methodological substudies. Fortunately, the Dutch Cancer Society has been supporting not only a two-year pilot study but also two four-year periods during which the actual cohort study was initiated and carried out so far. The methodological substudies, some of which have been financed by our own institutes, included testing the feasibility of a cohort study in the Netherlands, developing and testing the dietary questionnaire both in terms of validity and reproducibility, development of the record linkage for cancer follow-up and development of methods for statistical analysis of case-cohort studies. Conducting such methodological investigations has several advantages for the cohort study itself. They can be viewed as prerequisites for the interpretation of the results of the main study, because they give information on the quality of the various aspects of the study. This information can also be useful when the data from the cohort study are being used together with those of other studies in pooling or meta-analyses. We are currently participating in a pooling project on diet and various cancers in which data from five North American cohorts, one Swedish cohort and our cohort are analyzed in a collaborative effort. Particularly with regard to less frequent tumor sites, such an approach can, in addition to the individual studies, yield more definitive information on the relationship between diet and human cancer.

This thesis contains the first results of analyses of a number of diet-cancer relationships, conducted after 3.3 years of follow-up. With continued follow-up of the cohort, the study can be extended to the analyses of various other associations between diet (nutrients, non-nutritive factors, foods, dietary patterns) and the risk of common as well as rare cancers. Also, interactions between dietary factors or between diet and other factors (e.g., smoking, occupation) in relation to cancer risk can be investigated in more detail.

\section{References}

1. Sackelt DL, Haynes RB, Gouyat GH, Tugwell P. Clinical Epidemiology. A basic science for clinical medicine. Boston: Litile, Brown and Company, 1991.

2. Miettinen OS. Theoretical epidemiology. New York: Wiiey 1985.

3. Rothman KJ. Modern epideniology. Boston: Litule, Brown and Company 1986.

4. Rookus MA, Van Leeuwen FE. Misclassification and selection bias in a case-control study of breast cancer and oral contraceptives. (abstract). Am J Epidemiol 1992 (in press).

5. Jensen $O M$, Wahrendorf $J$, Rosenqvisi $A$, Geser $A$. The reliability of questionnaire-derived historical dietary information and temporal stability of food habits in individuals. Am $y$ Epidemiol 1984: 120: 281.290 .

6. Byers T, Marshall J, Anthony E, Fiedler R, Zielezny M. The reliability of dietary history from the distant past. Am J Epidemiol 1987; 125: 999-1011.

7. Wu ML. Whittemore AS, Jung DL Errors in reported dietary intakes, II. Long-term recall. Am J Epidemiol 1988; 128: 1137-1145.

8. Willett W. Nutritional cpidemiology. New York: Oxford University Press, 1990.

9. Giovanucci E, Stampfer MJ, Colditz GA, et al. A comparison of prospective and retrospective assessments of diet in the study of breast cancer (abstract). Am J Epidemiol 1991; 134: 714. 
10. Friedenreich $\mathrm{CM}$, Howe GR, Miller $\mathrm{AB}$. The effect of recall bias on the association of calorieproviding nutrients and breast cancer. Epidemiology 1991; 2: 424-429.

11. Willett WC, Stampfer MU, Colditz GA, Rosner BA, Hennekens CH, Speizer FE. Dietary fat and risk of breast cancer. N Engl J Med 1987; 316: 22-28.

12. Rimm EB, Giovannucci E, Willett WC, et al. Prospective study of alcohol consumption and risk of coronary disease in men. Lancet $1991 ; 338: 464-468$.

13. Maclure KM, Hayes KC, Colditz GA, Stampfer MJ, Speizer FE, Willett WC. Weight, diet, and the risk of symptomatic gallstones in middle-aged women. N Engl J Med 1989; 321: 563-569.

14. Witteman JC, Willett WC, Stampfer MJ, et al. Relation of moderate alcohol consumption and risk of systemic hypertension in women. Am J Cardiol 1990; 65: 633-637.

15. Hernandez Avila M, Colditz GA, Stampfer MJ, Rosner B, Speizer FE, Willett WC. Caffeine, moderate alcohol intake, and risk of fractures of the hip and forearm in middle-aged women. Am I Clin Nutr 1991; 54: 157-163.

16. Howe GR, Friedenreich CM, Jain M, Miller AB. A cohort study of fat intake and risk of breast cancer. J Natl Cancer Inst 1991; 83: 336-340.

17. Van Noord PAH, Collette HJA, Maas MJ, De Waard F. Selenium levels in nails of premenopausal breast cancer patients assessed prediagnostically in a cohort-nestedi case-referent study among women screened in the DOM project. Int J Epidemiol 1987; 16(suppl): 318-322.

18. Thorogood M, Carter R, Benfield L, McPherson K, Mann Jl. Plasma lipids and lipoprotein cholesterol concentrations in people with different diets in Britain. Br Med J 1987: 295: 351-353.

19. Vandenbroucke JP. Het Centraal Bureau voor de Statistiek: de begraafplaats van onze doodsoorzaken. Ned Tijjschr Geneeskd 1989; 133: 2112-2114.

20. Pietinen P, Hartman AM, Haapa E, et al. Reproducibility and validity of dietary assessment instruments: a self-administered food use questionnaire with a portion size picture booklet. Am J Epidemiol 1988; 128: 655-666.

21. Van Beresteyn ECH, van 'i Hof MA, van der Heiden-Winkeldermaat JHJ, ten Have-Witjes A, Neeter R. Evaluation of the usefulness of the cross-check dietary history method in bngitudinal studies. J Chron Dis 1987; 40: 1051-1058.

22. Walker AM, Blettner M. Comparing imperfect measures of exposure. Am J Epidemiol 1985; 121: 783-790.

23. Freedman LS, Schatzkin A, Wax Y. The impact of dietary measurement error on planning sample size required in a cohort study. Am J Epidemiol 1990; 132: 1185-1195.

24. Bausch-Goldbohm RA, van den Brandt PA, van 'I Veer P, van Faassen A, Hermus RJJ, Sturmans F. Diet and cancer of the breast, colon, rectum, stomach and lung: a pilot study for a prospective cohort study. Progress Report nr. 2. Zcist/Maastricht, 1985.

25. Van Faassen A, van 't Veer P, Goldbohm RA et al. Self-assessment of faecal pH and faccal bulk in epidemiological studies. Br J Cancer 1992; 65: 735-736.

26. Schatzkin A, Freedman LS, Schiffman MH, Dawsey SM. Validation of intermediate end points in cancer research. J Natl Cancer Inst 1990; 82: 1746-1752.

27. Hulka BS, Margolin BH. Methodological issues in epidemiologic studies using biologic markers. Am J Epidemiol 1992; 135: 200-209.

28. Knekt P, Aromaa A, Maatela J, et al. Serum selenium and subsequent risk of cancer among Finnish men and women. J Natl Cancer Inst 1990; 82: 864-868.

29. Comstock GW, Bush $\mathrm{T}_{\mathrm{h}}$, Hetzlsouer $\mathrm{K}$. Serum retinol, beta-carotene, vitamin $\mathrm{E}$, and selenium as related to subsequent cancer of specific sites. Am J Epidemiol 1992; 135: 115-121.

30. Alfthan G, Arno A, Arvilommi H, Iluttunen JK. Deposition of selenium in toenails in dependent on the from of dietary selenium. In: Kok FJ, Van ' 1 Veer P, eds. Biomarkers of dietary exposure. Proceedings of the 3rd meeting on Nutritional Epidemiology. London: Smith-Gordon, 1991, pp 110.

31. Hunter DJ, Morris JS, Stampfer MJ, Colditz GA, Speizer FE, Willett WC. A prospective study of selenium status and breast cancer risk. JAMA 1990; 264: 1128-1131.

32. Coates RJ, Weiss NS, Daling JR, Morris JS, Labbe RF. Scrum levels of selenium and retinol and the subsequent risk of cancer. Am J Epidemiol 1988; 128: 515-523.

33. Willett WC, Stampfer MJ. Selenium and cancer. Br Med J 1988; 297: 373-374.

34. Combs GF. Selenium. In: Moon TE, Micozzi MS, eds. Nutrition and cancer prevention; investigating the role of micronutrients. New York: Marcel Dekker, 1989: 389-420.

35. Lockitch G. Selenium: clinical significance and analytical concepts. Crit Rev Clin Lab Sci 1989; 27: 483-541.

36. Varo $P$, Affthan G, Ekholm P, Aro A, Koivistoinen P. Selenium intake and serum selenium in Finland: effects of soil fertilization with selenium. Am J Clin Nutr 1988; 48: 324.329. 
37. Van 't Veer P, van der Wielen RP, Kok FJ, Hermus RJ, Sturmans, F. Selenium in diet, blood, and toenails in relation to breast cancer: a case-control study. Am J Epidemiol 1990; 131: 987-994.

38. Van den Brandt PA, Goldbohm RA, Van 't Veer P, Bode P, Hermus RJ, Sturmans F. Predictors of toenail selenium levels in men and women. Cancer Epidemiol Biomark Prev (in press).

39. Hebzlsouer KJ. Selenium and cancer prevention. Semin Oncoll 1983; 10: 305-310.

40. Medina D. Mechanisms of selenium inhibition of tumorigenesis. J Am Coll Toxicol 1986; 5: 21-28.

41. Nakayama T, Kaneko M, Kodama M, Nagata C. Cigarette smoke induces DNA single-strand breaks in human cells. Nature $1985 ; 314: 462-464$.

42. Church DF, Pryor WA. Free-radical chemistry of cigarette smoke and its toxicological implications. Environ Health Perspect 1985; 64: 111-126.

43. Rogers MA, Thomas DB, Davis $\mathrm{S}$, Weiss NS, Vaughan Th, Nevissi AE. A case-control study of oral cancer and pre-diagnostic concentrations of selenium and zinc in nail tissue. Int J Cancer 1991; 48: 182-188.

44. Willett WC, Morris JS, Pressel S, et al. Prediagnostic serum selenium and risk of cancer. Lancet 1983; ii: $130-134$.

45. Salonen J,, Salonen R, Lappetelainen R, Maenpaa PH, Alfthan G, Puska P. Risk of cancer in relation to serum concentrations of selenium and vitamins $A$ and $E$ : matched case-control analysis of prospective data. Br Med J 1985; 290: 417-420.

46. Kok Fj, de Bruijn AM, Hofman A, Vermeeren R, Valkenburg HA. Is serum selenium a risk factor for cancer in men only? Am J Epidemiol 1987; 125: 12-16.

47. Hunter DJ, Morris JS, Chute CG, et al. Predictors of selenium concentration in human toenails. Am J Epidemioll 1990; 132: 114-122.

48. Chen J, Clark LC. Proposed supplemental dosages of selenium for a phase I trial based on dietary and supplemental selenium intakes and episodes of chronic selenosis. J Am Coll Toxicoll 1986; 5: $71-78$,

49. Clark LC, Combs GF. Selenium compounds and the prevention of cancer: research needs and public health implications. J Nutr 1986; 116: 170-173.

50. Yu SY, Mao BL, Xiao P, et al. Intervention trial with selenium for the prevention of lung cancer among tin miners in Yunnan, China. A pilot study. Biol Trace Elem Res 1990; 24: 105-158.

51. Xuan XZ, Schatzkin A, Mao BL, Taylor PR, Li JY, Tangrea J, Yao SX, Qiao YL, Giffen C, McAdams M. Feasibility of conducting a lung-cancer chemoprevention trial among tin miners in Yunnan, P. R. China. Cancer Causes Control 1991; 2: 175-182.

52. Broghamer WL, McConnell KP, Blotcky AL. Relationship between serum selenium levels and patients with carcinoma. Cancer 1976; 37: 1384-1388.

53. Rizk SL, Sky-Peck HII. Comparison between concentrations of trace elements in normal and neoplastic human breast tissue. Cancer Res 1984; 44: 5390-5394.

54. Prentice RL. Covariate measurement erions and parameter estimation in a failure time regression model. Biometrikal 1982; 69: 331-342.

55. Rosner B, Speigelman D. Willett WC. Correlation of logistic regression relative risk estimates and confidence intervals for measurement error: the case of multiple covariates measured with error. Am J Epidemiol 1990; 132: 734-745. 


\section{Summary}

Various types of cancer are thought to be related to dietary habits, but the epidemiologic evidence (mostly from case-control studies) is not always consistent. A prospective cohort study avoids at least one of the possible sources of inconsistency, namely retrospective assessment of dietary habits, which may introduce recall or information bias. However, required budget and time or feasibility considerations often preclude the conduct of such a study. In the Netherlands, the opportunity existed to efficiently recruit a cohort from the general population and, more importantly, to use the newly established cancer registries and PALGA (a computerized database of pathology reports) for follow-up. This thesis describes a prospective cohort study on dietary habits and the risk of cancer, conducted in the Netherlands since 1986 among more than 120,000 men and women. Methodological issues, such as the design of the study and the development and validation of methods used, form a substantial part of the thesis. First results after 3.3 years of follow-up are also presented.

The design of the prospective cohort study is presented in Chapter 2. In September 1986 , a cohort of 58,279 men and 62,573 women, aged 55-69, was recruited from 204 municipal population registries. The participants completed a self-administered questionnaire on dietary habits and potential confounders (e.g., smoking, occupation, education, reproductive variables); about two-thirds of them also provided toenail clippings, which were used to assess their selenium status. The initial interest was in stomach, colorectal, breast and lung tumors. To reduce costs, a case-cohort approach was applied in which a subcohort of 3500 subjects (1688 men, 1812 women) was randomly sampled from the cohort and followed up for vital status biennially to estimate the person-time accumulated in the entire cohort. The 204 municipalities were selected on the basis of two criteria: (a) availability of a computerized population registry - for practical reasons - and (b) sufficient coverage by cancer registries and/or PALGA to minimize loss to follow-up. The estimation of the coverage of the municipalities by these registries using hospital discharge data is being described in Chapter 3. It was estimated that the mean coverage degree of the cohort sample was $98.5 \%$ at the start of the study and was $100 \%$ complete in 1988.

Chapter 4 describes the development of a protocol, in collaboration with the IKLcancer registry, to link the cohort to the cancer data contained in the cancer registries and PALGA. The optimal procedure starts with a computerized linkage using a key consisting of encrypted information on the first four characters of the family name, date of birth and gender to match a cohort member to a cancer patient. Subsequent visual verification with additional identifying information (place: of birth, migration, first initial) is used to separate computer matches into true and false positive matches. In the pilot linkage with this protocol, a sensitivity of $98 \%$ was achieved, whereas the predictive value of (definitely) accepted matches was $100 \%$. The protocol has subsequently been adopted by all cancer registries and PALGA which have performed record linkages periodically.

An important aspect of this study was the assessment of usual dietary intake. In a pilot study in 1984-85 detailed dietary history interviews had been conducted among 169 men and women in the age range of the cohort. Regression analysis was employed to select from the data set those food items that contributed most to interindividual variation in intake of the 15 nutrients of primary interest. A total of 150 selected food items were incorporated in a semi-quantitative food frequency questionnaire, which was pretested twice. The validity and the reproducibility of the self-administered food 
frequency questionnaire (FFQ) are described in Chapters 5 and 6, respectively. The validity was investigated in a subgroup of the cohort ( 59 men and 50 women) two years after the baseline questionnaire was completed. A dietary record, kept over three 3-day periods, four to five months apart, served as reference method. Pearson correlation coefficients between nutrient intakes assessed by the record and the FFQ completed afterwards ranged from 0.40 for vitamin B-1 to 0.86 for alcohol intake, with correlations for most nutrients between 0.6 and 0.8. Adjustment for energy intake and sex did not materially affect these correlations, except that for fat intake, which changed from 0.72 to 0.52 . To evaluate the representativeness of the validation study population for the entire cohort, their baseline questionnaires were compared to those of a random sample of the cohort. Correlation coefficients were only slightly modified when the results were extrapolated to the cohort at large.

The reproducibility of the FFQ was determined from five annually repeated questionnaire administrations in independent random samples from the cohort. Pearson correlation coefficients between the baseline and the repeated measurement of nutrient intake were calculated for each time interval, i.e. ranging from one to five year. Linear regression of the correlation coefficients on time interval provided estimates of the testretest correlation of the FFQ (intercept of the regression line) and of the decline in correlation over time (slope). The test-retest correlation averaged over all nutrients was 0.66. The decline in correlation amounted on average to 0.07 after five years. It was concluded that the food frequency questionnaire is able to rank subjects according to intake of food groups and nutrients and that this capacity was maintained over time. Thus, a single baseline measurement with the FFQ is a good indicator of dietary habits over a period of at least five years.

Besides exposure information obtained by questionnaire, we also collected toenail clippings as a biomarker of the nutritional status of certain trace elements, in particular selenium. Toenail selenium levels and questionnaire data of subcohort members were used to identify potential determinants of toenail selenium in men and women, which may act as potential confounders in subsequent analyses of selenium and cancer risk (Chapter 7). Toenail selenium data were available for 2459 subcohort members. This analysis revealed that smoking, gender and selenium intake were independently associated with toenail selenium levels, whereas age, alcohol intake and Quetelet index were not. Current smokers showed lower selenium levels than non-smokers or past smokers; men showed lower levels than women. Selenium intake was weakly positively associated with toenail selenium levels.

When collection of biomarkers on a large scale is not feasible, biomarkers may be used to validate other exposure measurements. In this respect, nitrate levels from two overnight urine specimens were compared with nitrate intake information obtained by a semiquantitative food frequency questionnaire in a comparable cohort study on diet and cancer in the United States (Chapter 8). After correction for within-person variation in urinary excretion, the partial coefficient between dietary and urinary nitrate was 0.59 . These data suggested that a self-administered questionnaire may provide useful information on usual nitrate intake. In addition, these results were used in the construction of the Dutch cohort questionnaire.

In case-control studies, cancer cases may recall previous dietary habits (i.e., before diagnosis) differently from control subjects without cancer, leading to information bias. One of the reasons this might occur is that dietary habits were altered due to the disease. To test this assumption, we compared a cross-sectional analysis of the association between meat consumption and the prevalence of breast or colorectal cancer (both at the time of baseline measurement in the cohort) with a longitudinal analysis taking timing of a change in meat consumption frequency in relation to the 
date of cancer diagnosis into account (Chapter 9). These analyses revealed that meat consumption decreased substantially after cancer is diagnosed, which complicates the interpretation of cross-sectional and possibly case-control studies, since the recall of past dietary habits is influenced by current habits.

The method of statistical analysis of case-cohort studies is presented in Chapter 10. The chapter concentrates on methods for stratified analysis and regression analysis. The proposed methods were illustrated with the analysis of a well-known association in cancer epidemiology, namely that between smoking habits and lung cancer. The casecohort analysis of our cohort data confirmed the strong positive dose-response relationship between smoking and lung cancer. Compared to never smokers, the incidence rate of lung cancer was estimated to be 3.6 and 9.8 times higher for past and current smokers, respectively.

In Chapters 11-17, analyses of diet and cancer relationships are presented. For this thesis cancer follow-up data are used from the period September 1986-December 1989. In this 3.3 year period, the following numbers of microscopically confirmed incident cancer cases were detected in the cohort: 155 stomach, 313 colon, 166 rectal, 471 breast and 552 lung cancer cases. Regarding person-time, there were no subcohort members lost to follow-up in these 3.3 years.

Chapter 11 deals with the association between dietary fat and breast cancer. For this analysis dietary data were available for 437 incident breast cancer cases. No significant associations with breast cancer risk were detected for total fat, monounsaturated or polyunsaturated fat. For saturated fat, there was some evidence for a. weak positive association but its statistical significance was inconsistent. While this analysis did not support a major role of dietary fat in the etiology of breast cancer, it confirmed previously reported associations with nondietary breast cancer risk factors. Elevated risks were found for women with a family history of breast cancer, a history of benign breast disease, an early menarche and a late menopause, while decreased risks were observed for those with an early first pregnancy and high parity.

Chapters 12 and 13 describe the relationship between colorectal cancer risk and the consumption of alcohol and meat respectively. After exclusion of cases diagnosed in the first year of follow-up, the analysis of alcohol consumption was based on 217 incident cases of colon cancer and 113 cases of rectal cancer. For colon cancer, no association with intake of alcohol nor with the consumption of beer or wine could be demonstrated; for liquor a statistically significant decreasing risk with increasing consumption was observed. For rectal cancer in men, positive trends were observed for alcohol, beer and liquor. Multivariate models including alcohol intake and one beverage type at a time showed that the increased risk was mainly restricted to consumption of beer; the relative rate (RR) of beer drinkers compared to non-beer drinkers was 1.9. Results for rectal cancer in women were consistent with those in men, but data were too scarce to provide stable estimates. It was concluded that only consumption of beer appeared to increase risk of rectal cancer, but not colon cancer. It was speculated whether the high nitrosamine content of beer in the past has caused the increased risk.

The association between the consumption of meat and cancer of the colon was based on 215 incident cases, excluding those diagnosed in the first year of follow-up. No trends in relative rates were detected for intake of energy nor for energy-adjusted intake of fats, protein, fat from meat and protein from meat. Consumption of (fresh) meat, beef, pork, minced meat, chicken and fish was not associated with risk of colon cancer either. Meat products, however, were shown to increase risk in men and women ( $R R=1.17$ per $15 \mathrm{~g} /$ day). 
Risk of colorectal carcinoma after previous gallbladder surgery was investigated in 478 incident cases of colorectal cancer, 64 of whom reported at baseline to have undergone previous gallbladder surgery (Chapter 14). The relative rate for colorectal cancer in subjects who had undergone cholecystectomy compared to subjects who had not resulted in an RR of 1.8 in men and 1.5 in women. In women, the highest RR (1.9) was detected in the right colon, whereas in men, no site within the large bowel accounted specifically for the increased relative rate. In both men and women, the relative rate appeared to increase from approximately six years after cholecystectomy onward. According to the TNM stage of the disease, cholecystectomized patients were not detected in an earlier stage than the other patients. It was concluded that the positive association between colorectal cancer and cholecystectomy could not be explained by detection bias or ascertainment bias and was not confounded by risk factors for gallstone disease or by dietary factors.

In Chapters 15-17 results are presented on the association between toenail selenium and the risk of lung, stomach, colon, rectal and breast cancer. Toenail selenium data were available for 370 incident lung, 104 stomach, 234 colon, 113 rectal and 355 breast cancer cases. After controlling for smoking and various other factors, a significant inverse association was observed between toenail selenium levels and lung cancer risk (Chapter 15), with a RR of 0.50 for those in the highest selenium quintile compared to those in the lowest quintile. The inverse association was found in both men and women and persisted after excluding cases diagnosed in the first year of follow-up. The protective effect of selenium was concentrated in subjects with a low intake of Bcarotene and, in particular, vitamin C. This effect modification by vitamins with antioxidant properties supports the hypothesized anticarcinogenic effect of selenium based on its role in the cellular antioxidant system. For stomach cancer, suggestive, but inconsistent, evidence was found for an inverse relationship with toenail selenium $(R R=0.6$ for highest vs. lowest selenium category). The effect was only seen in men $(\mathrm{RR}=0.4)$, but the number of female cases was too small to draw more definitive conclusions about a possible gender specificity of the effect (Chapter 16). Although there was some evidence for modification of the effect of selenium on stomach cancer risk by the level of vitamin $\mathrm{C}$ intake, this was less clear than for lung cancer. This may be related to differences in etiology of lung and stomach cancer and in the importance of smoking as a risk factor. For colon and rectal cancer, no association was found between toenail selenium status and cancer risk. As for colon cancer (but not for lung, stomach and rectal cancer), breast cancer cases diagnosed in the first year of follow-up showed lower toenail selenium levels than cases diagnosed in subsequent years, suggesting an influence of prediagnostic disease (Chapter 17). After taking this into account, there was no association between toenail selenium and breast cancer risk. The observations on the tumors studied are in line with those of other reports and suggest that selenium status is inversely related to cancers of the upper digestive tract, as well as to lung cancer, which are associated with smoking. Our observations on the possible influence of prediagnostic disease also illustrate the need to interpret case-control studies on selenium and certain tumors with caution because of the likelihood of information bias.

While this thesis is limited to results of several diet-cancer analyses after 3.3 years of follow-up, continued follow-up of the cohort will enable the investigation of various other associations between diet (nutrients, non-nutritive factors, foods, dietary patterns) and the risk of common as well as rare cancers. Also, interactions between dietary factors or between diet and other factors (e.g., smoking, occupation) in relation to cancer risk can be investigated in more detail. 


\section{Samenvatting}

Een aantal soorten kanker wordt in verband gebracht met voedingsgewoonten, maar resultaten van epidemiologisch onderzoek (meestal patiênt-controle onderzoeken) zijn niet altijd consistent. Een prospectief cohortonderzoek vermijdt tenminste één van de mogelijke bronnen van inconsistentie, namelijk het vaststellen van iemands voedingsgewoonten in het verleden dat tot vertekening van het onderzoeksresultaat kan leiden (informatiebias). De kosten, de tijdsduur en de praktische uitvoerbaarheid verhinderen echter meestal dat zo'n onderzoek wordt opgezet. In Nederland bestond gelegenheid om op een efficiēnte wijze een cohort uit de algemene bevolking samen te stellen en - van nog groter belang - voor het vaststellen van kanker gebruik te maken van de pas opgezette kankerregistraties en PALGA (een computerbestand van p.a.rapporten): Dit proefschrift beschrijft een prospectief cohortonderzoek naar de relatie tussen voedingsgewoonten en het risico voor het krijgen van kanker, dat sinds 1986 wordt uitgevoerd onder meer dan 120.000 Nederlandse mannen en vrouwen. Methodologische onderwerpen, zoals de onderzoeksopzet en de ontwikkeling en validering van methoden die in het onderzoek worden gebruikt, vormen een belangrijk onderdeel van het proefschrift. Tevens worden de eerste resultaten na een follow-up periode van 3,3 jaar gepresenteerd.

De opzet van het prospectief cohortonderzoek staat weergegeven in hoofdstuk 2. Het cohort omvat 58.279 mannen en 62.573 vrouwen van 55-69 jaar, afkomstig uit 204 gemeentelijke bevolkingsregisters. De cohortdeelnemers hebben in september 1986 een schriftelijke vragenlijst ingevuld over hun gewoonlijke voedselconsumptie en potentiële confounders (bijv. rookgewoonten, beroep, opleiding, medische informatie); circa tweederde van hen stuurde ook teennagelknipsels in, waarmee de seleniumstatus in het lichaam gemeten kan worden. In eerste instantie gaat de aandacht uit naar maligniteiten van de maag, colon en rectum, borst en long. Om kosten te besparen is een case-cohort benadering gebruikt waartoe aselect een subcohort van 3500 mensen (1688 mannen, 1812 vrouwen) is getrokken uit het cohort; door dit subcohort elke twee jaar aan te schrijven wordt ingeschat hoeveel persoonsjaren 'at risk' in het totale cohort worden opgebouwd. De 204 deelnemende gemeenten werden doelbewust gekozen op grond van twee criteria: (a) de aanwezigheid van een geautomatiseerd bevolkingsregister - om praktische redenen - en (b) voldoende dekking van de gemeente door de kankerregistraties en/of PALGA om een zo compleet mogelijke follow-up te bewerkstelligen. In hoofdstuk 3 wordt beschreven hoe de dekkingsgraad van de gemeenten door deze registraties is geschat met behulp van gegevens over ontslagdiagnoses uit ziekenhuizen. De gemiddelde dekkingsgraad van het cohort werd geschat op $98.5 \%$ bij de start van het onderzoek en op $100 \%$ in 1988 .

In hoofdstuk 4 wordt de ontwikkeling (i.s.m. het Integraal Kankercentrum Limburg) beschreven van een protocol voor de koppeling van de cohortgegevens met de gegevens van de kankerregistraties en PALGA. De optimale procedure gaat uit van een geautomatiseerde koppeling met een koppelingssleutel bestaande uit geëncrypteerde informatie over de cerste 4 letters van de geboortenaam, de geboortedatum en het geslacht, waarmee cohortleden gekoppeld kunnen worden aan kankerpatiënten. Vervolgens vindt handmatige verificatie hiervan plaats met additionele identificerende gegevens (geboorteplaats, migratie, eerste voorletter), waardoor de computerkoppelingen gescheiden kunnen worden in terecht en fout-positieve koppelingen. In een proefkoppeling werd met dit protocol een sensitiviteit van $98 \%$ bereikt, terwijl de predictieve waarde van (definitjef) geaccepteerde koppelingen $100 \%$ bedroeg. Dit. 
protocol is vervolgens overgenomen door alle regionale kankerregistraties en PALGA, die daarna periodiek koppelingen hebben uitgevoerd.

Een belangrijk onderdeel van het onderzoek was het vaststellen van de gebruikelijke voedingsgewoonten bij de leden van het cohort. Tijdens een vooronderzoek in 1984 en 1985 werden uitgebreide mondelinge voedingsenquêtes afgenomen bij 169 mannen en vrouwen in de leeftijd van het cohort. Met behulp van regressieanalyse werden uit het resulterende gegevensbestand die (groepen van) voedingsmiddelen geselecteerd die het meest bijdroegen aan de variantie in inneming van 15 nutriënten die van mogelijk belang geacht werden voor kanker. De 150 geselecteerde voedingsmiddelen werden verwerkt in een schriftelijke zogenaamde voedselfrequentielijst (food frequency questionnaire, FFQ), die tweernaal werd uitgetest.

De validiteit en de reproduceerbaarheid van de FFQ zijn respectievelijk beschreven in hoofdstukken 5 en 6. De validiteit is onderzocht in een subgroep uit het cohort (59 mannen en 50 vrouwen) twee jaar na de afname van de lijst bij de start van het cohortonderzoek. Gedurende drie perioden van drie opeenvolgende dagen, met tussenpozen van vier tot vijf maanden, werd door de deelnemers een gedetailleerd voedingsdagboek bijgehouden. De hieruit verkregen gegevens werden gebruikt als. referentie voor de voedselfrequentielijst, die twee maanden na de laatste opschrijfperiode nogmaals werd ingevuld. De Pearson correlatiecoëfficiënten tussen de opschrijfmethode en de FFQ varieerden van 0,40 voor de inneming van vitamine B-1 tot 0,86 voor de alcoholinneming; de meeste correlaties lagen tussen 0,6 en 0,8 . Correctie voor energieinneming en geslacht hadden weinig invloed op de correlatie, behalve voor vet, waarvan de correlatie daalde van 0,72 naar 0,52 . Om de representativiteit van de deelnemers aan het valideringsonderzoek ten opzichte van het gehele cohort te beoordelen, werden hun oorspronkelijke ("baseline") vragenlijsten vergeleken met die van een aselecte steekproef uit het cohort. De correlatiecoëfficiënten veranderden slechts weinig bij extrapolatie van de resultaten uit het valideringsonderzoek naar het gehele cohort.

De reproduceerbaarheid van de FFQ werd gemeten via vijf jaarlijkse herhalingen van de vragenlijst bij aselecte, onafhankelijke steekproeven uit het cohort. Pearson correlatiecoëfficiënten tussen de eerste en de herhaalde meting van de nutriëntinneming werden berekend voor ieder tijdsinterval variërend van een tot vijf jaar. Lineaire regressie van de correlatiecoëfficiënten op tijdsinterval leverdeln schattingen op van de test/hertest correlatie van de FFQ (intercept van de regressielijn) en van de afname van de correlatie in de tijd (helling, van de lijn). De test/hertest correlatie bedroeg gemiddeld 0,66. De gemiddelde afname in correlatie bedroeg 0,07 na vijf jaar. De conclusie is dat de voedselfrequentielijst in staat is personen te rangschikken volgens hun voedingsmiddelengebruik en nutriëntinneming en dat blijft over een periode van tenminste vijf jaar. Een enkele meting met behulp van de FFQ geeft dus een goede indicatie van de voedingsgewoonten gedurende langere tijd.

Behalve vragenlijstinformatie over de blootstelling zijn ook teennagelknipsels verzameld als "biomerker" van de lichaamsstatus van een aantal sporenelementen, met nane selenium. Seleniumgehalten in teennagels en vragenlijstgegevens van de mensen uit het subcohort zijn gebruikt om potentiële determinanten van teennagelseleniumgehalten in mannen en vrouwen op te sporen, die als mogelijke confounders op kunnen treden in de analyse van selenium en kankerrisico (hoofdstuk 7). In totaal waren van 2459 subcohortleden seleniumgehalten in nagels beschikbaar. Deze analyse liet zien dat roken, geslacht en seleniumconsumptie onafhankelijk van elkaar waren gerelateerd aan seleniumwaarden van de nagels; dat gold niet voor 
leeftijd, alcohol en Quetelet index. Rokers vertoonden lagere seleniumgehalten dan exrokers of personen die nooit gerookt hadden; mannen lieten lagere seleniumwaarden zien dan vrouwen.

Als biomerkers niet op grote schaal verzameld kunnen worden, kunnen ze eventueel wel dienen om andere blootstellingmetingen te valideren. Als illustratie hiervan werden nitraatgehalten van 2 urinemonsters, telkens verzameld gedurende de avond en nacht, vergeleken met de nitraatconsumptie zoals gemeten met een semikwantitatieve voedselfrequentievragenlijst in een gerelateerd Amerikaans cohortonderzoek naar voeding en kanker (hoofdstuk 8). Na correctie voor binnenpersoons-variatie in de urineuitscheiding bedroeg de partiële correlatiecoëfficiēnt tussen nitraat uit de voeding en urine 0,59 . Uit deze bevindingen was af te leiden dat met een schriftelijke vragenlijst bruikbare informatic over de gewoonlijke nitraatconsumptie is te verkrijgen. Tevens konden deze resultaten gebruikt worden bij de constructie van de Nederlandse cohortvragenlijst.

In patiënt-controleonderzoek kan informatiebias ontstaan wanneer kankerpatiènten zich vroegere voedingsgewoonten (van vóór de diagnose) anders herinneren dan controlepersonen zonder kanker. Dit kan onder andere gebeuren doordat voedingsgewoonten gewijzigd kunnen zijn als gevolg van de ziekte. Om deze aanname te verifiëren vergeleken we een cross-sectionele analyse van het verband tussen vleesconsumptie en de prevalentie van borst of darmkanker (beide ten tijde van de baselinemeting in het cohort) met een longitudinale analyse waarin het tijdstip van verandering in vleesconsumptie in relatie tot het tijdstip van kankerdiagnose verdisconteerd werd (hoofdstuk 9). Uit deze analyses bleek dat de frequentie van vleesconsumptie in kankerpatiēnten substanticel gedaald is na de kankerdiagnose. Dit bemoeilijkt de interpretatie van dwarsdoorsnede- en mogelijk ook patiëntcontroleonderzoek, aangezien de herinnering van vroegere gewoonten beïnvloed wordt door de huidige eetgewoonten.

In hoofdstuk 10 is de methode gepresenteerd om case-cohortonderzoeken statistisch te analyseren. Daarbij wordt aandacht geschonken aan methoden voor gestratificeerde analyse en regressieanalyse. De voorgestelde methoden werden geillustreerd met een analyse van een bekende relatie in de kankerepidemiologie, namelijk die tussen rookgewoonten en longkanker. Onze case-cohortanalyse bevestigde de sterk positieve dosis-responsrelatie tussen roken en longkanker. Ten opzichte van nooit-rokers werd het relatief risico (in feite: relatieve rate, $R$ ) op longkanker voor vroegere en huidige rokers geschat op respectievelijk 3,6 en 9,8 .

In de hoofdstukken 11-17 wordt ingegaan op resultaten uit analyses van enkele verbanden tussen voeding en kanker. Hierbij zijn follow-up gegevens gebruikt uit de periode van september 1986 tot en met december 1989. Giedurende deze 3,3 jaren van follow-up werden de volgende aantallen microscopisch bevestigde incidente kankerpatiënten gedetecteerd in het cohort: 155 maag-, 313 colon-, 166 rectum-, 471 borst- en 552 longkankerpatiënten. De follow-up van subcohortleden (persoonsjaren) was compleet.

In hoofdstuk 11 wordt de analyse van het verband tussen voedingsvet en borstkanker gepresenteerd. Voor deze analyse waren voedingsgegevens beschikbaar van 437 incidente borstkankerpatiënten. Er werden geen significante verbanden gevonden tussen het risico op borstkanker en de consumptie van totaal vet, enkelvoudig- en meervoudig onverzadigd vet. Verzadigd vet leek zwak positief geassocieerd te zijn met borstkanker maar de statistische significantie van deze bevinding was niet consistent. Teruijl deze analyse geen ondersteuning bood voor de hypothese dat vet sterk 
gerelateerd is aan borstkanker, bleken een aantal traditionele risicofactoren ook in ons onderzoek gerelateerd te zijn aan het borstkankerrisico. Verhoogde risico's werden waargenomen voor vrouwen met een positieve familieanamnese voor borstkanker, een goedaardig borstgezwel in de voorgeschiedenis, een vroege menarche en een late menopauze; verlaagde risico's werden gevonden voor vrouwen met een vroege eerste zwangerschap en een hoge pariteit.

Hoofdstukken 12 en 13 beschrijven het verband tussen het risico op dikke darm kanker enerzijds en het gebruik van respectievelijk alcohol en vlees anderzijds. De analyse van alcoholgebruik was, na uitsluiting van patiënten die gediagnostiseerd werden in het eerste jaar na de start van het onderzoek, gebaseerd op respectievelijk 217 en 113 inciđente colon- en rectumkankerpatiënten. Voor colonkanker werd geen verband gevonden met alcoholgebruik, noch met de consunptie van bier en wijn; voor jenever werd een met de gebruikte hoeveelheid afnemend risico geconstateerd. Voor rectumkanker bij mannen werd een positief verband met alcohol, bier en jenevergebruik gevonden. Multivariate modellen, waarin alcohol en één soort drank steeds tegelijk: opgenomen werden, maakten aannemelijk dat het verhoogde risico voornamelijk. beperkt is tot biergebruik; het relatieve risico (RR) voor bierdrinkers vergeleken met niet-bierdrinkers was 1,9 . De resultaten voor rectumkanker bij vrouwen kwamen

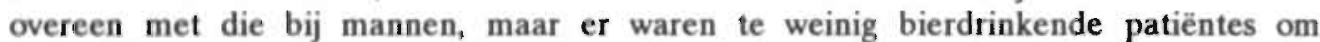
betrouwbare schattingen te maken. Mogelijk, doch onbewezen, heeft het hoge gehalte aan nitrosaminen dat vroeger in bier voorkwam bijgedragen aan het verhoogde risico.

De analyse van het verband tussen vleesconsumptie en colonkanker was gebaseerd op 215 incidente patiènten na uitsluiting van patiënten gediagnostiseerd in het eerste jaar. Er werd geen verband aangetoond met de inneming van energie en voor energie gecorrigeerde inneming van vetten, eiwit en van vlees afkomstig vet en eiwit. Ook de consumptie van (vers) vlees, rundvlees, varkensvlees, gehakt, kip en vis bleek niet. geassocieerd te zijn met het risico op colonkanker. Vleeswaren gaven echter een verhoogd risico te zien, zowel bij mannen als bij vrouwen ( $R R=1,17$ per $15 \mathrm{~g} / \mathrm{dag}$ ).

Het risico op dikke darmkanker na en galblaasoperatie is onderzocht bij 478 patiënten, waarvan 64 in de vragenlijst hadden aangegeven die operatie ondergaan te hebben (hoofdstuk 14). Bij mannen en vrouwen kwam respectievelijk 1,8 en 1,5 maal zo vaak darmkanker voor na een galblaasoperatie. Bij vrouwen werd het hoogste relatieve risico (1,9) gevonden voor het proximale deel van het colon, terwijl bij mannen geen verschil tussen localisaties kon worden aangetoond. Zowel bij mannen als bij vrouwen deed de stijging van het relatieve risico zich pas voor ongeveer zes jaar na de operatie. Darmkankerpatiënten die eerder aan de galblaas waren geopereerd werden niet in een vroeger stadium gediagnostiseerd dan de overige patiënten. De conclusie luidt dat het waargenomen positieve verband tussen cholecystectomie en darmkanker niet verklaard wordt door een aantal bekende bronnen van vertekening noch door samenhang met risicofactoren voor galstenen of met voedingsvariabelen.

In de hoofdstukken $15-17$ zijn de resultaten gepresenteerd van het verband tussen seleniumgehalten in teennagels en het risico op long-, maag-, colon-, rectum- en borstkanker. Voor deze analyses waren seleniumwaarden beschikbaar van 370 incidente longkankerpatiënten, 104 maag, 234 colon, 113 rectum en 335 borstkankerpatiënten. Deze analyses lieten, na controle voor roken en enkele andere factoren, een significant negatief verband zien tussen teennagelselenium en het risico op longkanker (honfdstuk: 15); het relatieve risico voor degenen uit het hoogste quintiel van selenium ten opzichte van het laagste quintiel bedroeg 0,5. De negatieve associatie werd zowel bij mannen als bij vrouwen waargenomen en bleef bestaan nadat patiènten waren uitgesloten die in het 
eerste jaar van follow-up waren gediagnostiseerd. Het beschermende effect van selenium werd vooral aangetroffen bij mensen met een lage inneming van beta-caroteen en met name vitamine C. Deze effectmodificatie door vitaminen met antioxidanteigenschappen ondersteunen de hypothese dat selenium anticarcinogeen kan werken via zijn rol in het cellulaire verdedigingssysteem tegen oxydatieve schade. Voor maagkanker werd ook een negatieve associatie met selenium gevonden maar de bevindingen waren minder consistent ( $R R=0,6$ voor hoogste versus laagste selenium categorie). Het effect werd alleen bij mannen waargenomen $(R R=0,4)$, maar het aantal vrouwelijke maagkankerpatiënten was te gering om meer definitieve conclusies te trekken over een mogelijke geslachtsspecifiek effect (hoofdstuk 16). Hoewel er bij maagkanker ook enige aanwijzing was voor een effectmodificatie van het seleniumeffect door de vitamine C consumptie, was dit minder duidelijk dan voor longkanker. Mogelijk heeft dit te maken met verschillen qua etiologie tussen long- en maagkanker en het belang van roken daarin. Voor colon- en rectumkanker werd geen verband gevonden tussen seleniumconcentratie in teennagels en het kankerrisico. Evenals voor colonkanker (maar niet voor long-, maag- en rectumkanker) vertoonden borstkankerpatiēnten die in het eerste jaar van follow-up gediagnostiseerd werden, lagere seleniumwaarden in de nagels dan patiènten uit latere follow-up jaren, hetgeen op een invloed van preklinische ziekte wijst (hoofdstuk 17). Wanneer hiermee rekening werd gehouden bleek er geen verband te bestaan tussen seleniumwaarden en het risico op borstkanker. De waarnemingen ten aanzien van deze kankervormen komen overeen met bevindingen uit andere onderzoeken en suggereren dat selenium negatief gerelateerd is aan kanker van het bovenste deel van de tractus digestivus, alsmede aan longkanker, kankervormen die geassocieerd zijn met roken. Onze bevindingen ten aanzien van de mogelijke invloed van preklinische kanker illustreren tevens dat patiënt-controleonderzoeken naar selenium en bepaalde kankervormen voorzichtig moeten worden geïnterpreteerd vanwege de mogelijke aanwezigheid van informatiebias.

Terwijl dit proefschrift zich beperkt tot de eerste resultaten van een follow-up periode van 3,3 jaar, zullen na continuering van de follow-up ook andere associaties tussen voeding (voedingsstoffen, andere bestanddelen van de voeding, voedingsmiddelen, voedingspatronen) en het risico op zowel frequente als zeldzame kankervormen onderzocht kunnen worden. Ook kunnen dan interacties tussen voedingsfactoren of tussen voeding en andere factoren (bijvoorbeeld roken, beroep) met betrekking tot kankerrisico meer in detail worden bestudeerd. 



\section{Dankwoord}

Het zal duidelijk zijn dat ook dit proefschrift niet alleen het werk is van de promovendi. Op de eerste plaats willen we hierbij alle deelnemers aan het cohortonderzoek hartelijk danken voor het invullen van de vragenlijst en insturen van teennagels in 1986. Hoewel we uit vooronderzoek hadden geschat dat het invullen ongeveer één uur in beslag zou nemen, hebben we later nog vaak kunnen vernemen dat het ook veel langer kan duren. Ook heeft $2 / 3$ van de deelnemers zelf voor postzegels voor de retourzending gezorgd en daarmee $f 120.000,-$ bespaard aan onderzoeksgeld. Hierbij willen we vooral ook de leden van het subcohort danken voor hun inzet om regelmatig een vervolgvragenlijst in te vullen, waarmee cruciale informatie werd verkregen voor het onderzoek. We hopen dat we dit ook in de toekomst nog voort mogen zetten.

Ten tweede willen we onze promotoren danken voor hun bijdragen. Ferd, jij hebt ons steeds en op diverse wijzen gesteund bij het uitvoeren van onze ideeën. Vooral in het begin van 1986 was jouw hulp en daarmee die van de RU Limburg van groot belang voor het daadwerkelijk starten van het onderzoek toen niet voor iedereen duidelijk was dat ook postzegels een essentieel onderdeel uit kunnen maken van wetenschappelijk onderzoek. We hopen tevens dat we je voldoende gecompenseerd hebben. Ook jouw steun, Ruud, is altijd van groot belang geweest om het project te continueren. ledere wetenschapper weet dat de beginjaren van een prospectief́ onderzoek moeizaam kunnen verlopen. Het duurt lang voordat er 'resultaten' komen en er moet zeer veel moeite gestoken worden in de opzet en uitvoering, zeker wanneer het op deze schaal wordt aangepakt. We stellen het erg op prijs dat jullie altijd vertrouwen in ons en de goede afloop hebben gehad, want het was toch wel een riskante onderneming.

We owe very special thanks to professor Walter Willett of the Marvard School of Public Health. You infected us with your enthusiasm for nutritional epidemiology and convinced us that conducting a prospective cohort study on diet and cancer wits not a mad idea. Your example and transatlantic support has been very important to us, in particular at times when other people were sceptic. We enjoy collaborating with you in the Pooling Project of seven prospective studies and look forward to the intellectually very stimulating discussions on the first analyses this summer. We also want to thank you very much for taking the time to come to Maastricht as a member of the Committee.

Vervolgens willen we graag Pieter van 't Veer en Elisabeth Dorant bijzonder bedanken voor hun inzet in de diverse fasen van het onderzoek. Pieter, jij was vanaf het begin bij de opzet van dit project betrokken. De eerste idecên voor een cohortonderzoek in Nederland zijn geboren in Shattuck House in Boston en namen daar, na vele lekkere door Matthea gekookte maaltijden, steeds reëler vormen aan. Aangezien je patiënt-controleonderzoek een steeds groter deel van je tijd ging opeisen, heb je je daar op toegelegd. Echter ook in de analysefase heb je ons gevraagde en ongevraagde adviezen kunnen geven die goed van pas kwamen. We hopen dat we deze samenwerking kunnen continueren nu je in Wageningen werkt. Elisabeth, ok jij vervulde een cruciale rol in het onderzoek, met name bij de follow-up via de kankerregistraties en PALGA. Jij weet als geen ander dat het uitvoeren van dergelijke koppelingen mooi klinkt maar desalniettemin nog zeer veel input vergt bij de interpretatie van hieruit voortkomende informatie. Daarnaast speelde je een belangrijke rol bij de vele ad boc vragen tijdens de invoer en bij het opzetten van de database. Net als wij zag jij in dat het uitvoeren van cohortonderzoek gewoon erg veel inzet vergt vooraleer er resultaten geboekt kunnen worden. Je moet er dan ook niet aan beginnen 
als je op korte termijn-successen uit bent. We zijn erg verheugd dat je in de komende tijd de gelegenheid krijgt ook jouw resultaten te boeken.

Henny Brants, jij kwam in 1984 voor het eerst als uitzendkracht voor ons project op TNO werken. Daar deed je, samen met Anneke Huldij, het veldwerk voor de pilot study. Nadat je een paar jaar de interviews voor het patiënt-controleonderzoek naar borstkanker had gedaan, heb je je ervaring ingezet voor supervisie en training van de diëtisten die het valideringsonderzoek uitvoerden. Daarna was (en is nog steeds) jouw voedingskundige inbreng bij het invoeren en het verwerken van de voedingsvragenlijst van groot belang. Je gedegen werkwijze, de manier waarop je alles tot in details documenteert, je vermogen om alle kleine en grote problemen te overzien zijn belangrijke schakels geweest in het succes van het voedingskundige deel van het onderzoek. Tot slot bleek je ook nog in staat je aan te passen bij onze kronkels, die wel. eens van de bestaande paden afweken.

Zeer veel mensen waren in de afgelopen negen jaar voor kortere of langere tijd bij dit onderzoek betrokken als medewerker, uitzendkracht of student, in Maastricht en. Zeist. We noemen hierbij in de eerste plaats de administratieve assistenten (in chronologische volgorde): Hans Smit, Judith Knipsael, Dave van Gelder, Mariëlle Bethlehem, Gemma Snijders, Patricia Florax, Arthur van Aken, Judith van den Brink, Jolanda Nelissen, Willy van Dijk en Annemie Pisters. Desirée van de Cappellen en Jenny Goes hebben in recordtempo een record aantal vragenlijsten ingevoerd. Het zoeken van de vragenlijsten en (nog erger) teennagels in die enge kelder vol spinnewebben, het invoeren van duizenden vragenlijsten, en het versturen van talloze mailings, jullie deden het allemaal. Zonder jullie inzet was er niets van het hele project terechtgekomen. Jullie hebben. soms heel wat van ons en onze ideeën te verduren gehad. Sacha van de Crommert, door jouw inzet in de verschillende fasen van het onderzoek werd de invoer in goede banen geleid en door jouw consequente manier van werken werd het datamanagement verder verbeterd. Arthur Schiphorst, van jou hebben wij kneepjes van het publiciteitsvak geleerd toen het onderzoek van start ging. De automatisering in het cohortonderzoek was natuurlijk geen sinecure. Gelukkig konden we hierbij gebruik maken van de diensten van het Medisch en Maatschappelijk Informatiecentrum van de RL en de rekencentra van beide instellingen. We willen hier graag Harry van Montfort, Ruud Schmeitz, Gregor Franssen, Marlène Kruijen en Tony van Montfort danken voor hun betrokkenheid bij dit onderzoek. Door belangrijke steun van professor Riet Drop (Medische Sociologie) werd het mogelijk orn de gegevensinvoer via een personal computer daadwerkelijk gestalte te geven. Ook waren je adviezen met betrekking tot de meting van alcoholconsumptie waardevol. Cor Kistemaker, jouw VEVES programma is zeer plezierig voor het laatste onderdeel van de vragenlijstverwerking. namelijk de berekening van autriënten en voedingsmiddelengebruik.

Van de vele studenten en uitzendkrachten noemen we Carla van Deursen en Hannelore Hofhuis, die de vragenlijst uittestte en de contacten met de drukker onderhield toen Sandra met zwangerschapsverlof was. Monique Al, Ingeborg van den Heuvel, Anita Langeveld, Renée Boogerd, Dian Drenth, Marieke Rouwhorst, Ellen van Vliet en Jacqueline Neijenhuis hebben tijdens het veldwerk voor het valideringsonderzoek heel wat fiets-, bus- en autokilometers afgelegd. Jeanne van Loon, het was een plezierige ervaring om met jou de analyses van de respons en vlees en kanker te verrichten; je tegenwoordige rol in het cohortonderzoek stellen we ook zeer op prijs. Hanneke den Breeijen, jij hebt, na je doctoraalvak, de spits afgebeten bij het verwerken van de voedingsvragenlijst, waarbij je bleek aan te voelen waarom mensen bepaalde invulfouten maakten. Stagiaires van de Hogere Informaticaopleiding in Heerlen (Jacqueline Vliegen, Helma van der Linden, Anky Gense, Michiel van Kessel, 
Marc Houben, Luc Crousen en Michel Koken) hebben allerlei nuttige programmatuur geschreven.

Lex Volovics en Marion de Leeuw willen we hartelijk danken voor hun bijdragen bij de opzet en de statistische analyse van het onderzoek. Martin van 't Hof, dankzij de uit de KRIS periode stammende contacten met jou hebben wij niet één maar wel vijf herhaalde metingen in het design geïncorporeerd en geanalyseerd. Ook zijn we Jet Smit en Svenneke van de Heuvel van het NIPG dankbaar voor de verwerking van beroepsgegevens. Voor het uitvoeren van de seleniumanalyses in circa 4000 nagels zijn we Peter Bode, Anneke Ammerlaan en Frank van Paassen van het Interuniversitair Reactor Instituut in Delft veel dank verschuldigd. Wat het aantal nagelanalyses betreft, zijn jullie momenteel vast en zeker wereldrecordhouders.

Vervolgens willen we natuurlijk stilstaan bij de zeer belangrijke rol die de kankerregistraties en PALGA hebben gespeeld in het tot stand komen van dit onderzoek. De (combinatie van) gegevens van beide soorten instellingen vormden voor ons precies datgene wat een groot cohortonderzoek mogelijk maakt in Nederland. PALGA vormt met zijn geautomatiseerde landelijke databank van pathologischanatomische gegevens een unieke gegevensverzameling. Wij zijn PALGA, tegenwoordig SIG-Amsterdam, zeer erkentelijk voor de bereidwilligheid om deze gegevens te gebruiken voor het cohortonderzoek en willen hierbij m.n. Renso Camps, Harmen Schut, Ben van den Bergh, Han Hol en Lodewijk. Otto danken voor hun medewerking en adviezen.

De Integrale Kankercentra zijn erin geslaagd om in relatief korte tijd een complete, landelijk dekkende kankerregistratie (de landelijke als som van de regionale registraties) op te zetten. Dit mag gezien de eerdere ervaringen in de jaren vijftig en zestig gerust een prestatie genoemd worden. Het is daarbij mogelijk. gebleven - ook in deze tijd van privacydiscussies - de gegevens te gebruiken voor o.a. epidemiologisch onderzoek. Wij willen alle regionale kankerregistraties van de verschillende Integrale Kankercentra (IKA, IKL, IKMN, IKN, IKO, IKR, IKST, IKW, IKZ), alsmede het Landelijk Overlegorgaan Kankercentra hartelijk danken voor hun bereidwilligheid mee te werken aan dit onderzoek.

Een zeer speciaal woord van dank gaat uit naar Leo Schouten van het Integraal Kankercentrum Limburg. Leo zag al erg vroeg het belang in van dit onderzoek, ook voor de kankerregistratie. Door de intensivering van onze contacten (via het IKLconsulentschap van één van ons) gaf hij ons een duidelijker inzicht in de werkwijze en ontwikkelingen in de registraties. Vooral door het gezamenlijk ontwikkelen van het. koppelingsprotocol werd de follow-up veel concreter en beter uitvoerbaar. Daarnaast. heeft Leo een belangrijke rol gespeeld bij het adopteren van het. protocol door de andere IKCs. Leo, het is helaas niet gelukt onze samenwerking verder formeel uit te bouwen, maar we hopen die in de toekomst toch yoort te zetten. Daarnaast mag ook de. rol van de andere Hoofden en automatiseringsdeskundigen van de kankerregistraties uit: de verschillende IKCs niet onvermeld blijven. We willen hierbij met name noemen: Tiny Benraadt en Otto Visser (IKA/IKST); Perry Hünen en Loek Smeets (IKL); Hans Berkel, Charles Gimbrère en Frits Bosman (IKMN); Renée Otter, Joyce Span en Jos Schakenraad (IKN); Bart Kiemeney en Herman Ament (IKO); Mary-Ann Fijn vạn Draat, Ronald Damhuis en Marja Tolsma (IKR); Hajo Kruijff, Roel van Westering en Harry Verschuur (IKW); Marijke Verhagen-Teulings en Louis van der Heijden (IKZ). De Nederlandse Vegetariërsbond, de VNR, Theo van Rooy van Smiths Reform en Frans van der Reep van Natufood willen wij bedanken voor hun hulp bij het recruteren van vegetariërs. Aan hen heeft. het zeker niet gelegen dat dit onderdeel niet zo'n succes werd. Ook willen we op deze plaats onze dank betuigen aan de leden van de 
Begeleidingscommissie en de Stuurgroep, de deelnemende gemeenten, de Stuurgroep Epidemiologie in de Basisgezondheidsdiensten, de Vereniging van Directeuren Basisgezondheidsdiensten, het Bureau Kwaliteitsaangelegenheden van TNO (J. Remmerswaal, M. Gruisen) en de gemeente Maastricht (met name J. Kikken) voor hun medewerking aan het onderzoek.

Verder willen we de (ex-)collega's van de vakgroep Epidemiologie en de afdeling Voeding danken voor de plezierige werksfeer en de hulp in voorkomende gevallen. Met name willen we hierbij noemen Ad Vissers, de stille ksacht die door zijn bufferende werking het project in goede banen hielp leiden; Diny van Faassen voor haar rol in de pilot-fase van het onderzoek en adviezen ten aanzien van biomarkers in bet algemeen en teennagels in het bijzonder; Frans Kok voor je steun en goede adviezen. Naast Thum 'Thesis' Aarts, die je over lay-out niets hoeft uit te leggen, zoals men kan zien, willen we ook Willy en Patricia speciaal bedanken voor hun bijdragen aan dit proefschrift en het ontcijferen van de manuscripten. Zoals jullie gemerkt hebben, was het goed voor je Grieks! Dirk van der Heij was daarentegen goed voor ons. Engels en werd vee! vaker dan "biennially" te hulp geroepen.

En, last but surely not least, zijn we Geja en Edward zeer dankbaar voor hun enorme steun vóór, tijdens en na dit onderzoek. Alleen julliie weten echt wat dit project aan ijjsinvestering heeft gekost, waardoor veel andere dingen erbij inschoten. Door jullie motivatie - en kennis van zaken - tijdens de moeilijker fasen bleven we in de goede afloop geloven. Jullie bijdragen waren ons het meest dierbaar. Bep van Vlooten en Ankie Gerrits, jullie waren onovertroffen "kunstmoeders" voor Sandra's kinderen. Dankzij jullie liefde en huishoudcapaciteiten heeft de hele familie de afgelopen acht jaar uitstekend doorstaan. 
Please answer these questions as indicated in the example on the explanation form, preferably with pencil.

If this is not possible, you may use a blue or black pen.

The answering possibilities have a white background.

C TNO-AL, august 1986

1 Here your date of birth is printed, as given by you or your municipality:

Is this date correct? $\square$ yes ( $g 0$ on to question 2) $\square$ no

If not, are you the the person whose name and address are printed on the envelope?

$\square$ yes $\rightarrow$ If so, what is your correct date of birth? $\square \square-\square \square-19 \square \square$

no $\rightarrow$ If so, could you give this letter to the addressee?

2 In what municipality were you born? ___ (country)

3 What is your marital status? $\square$ never married $\square$ divorced

4 Do (or did) you have twin brother? $\square$ no $\square$ yes

If so, is his first initial the same as yours? $\square$ no $\square$ yes

$\begin{array}{clll}5 \text { Do you smoke currently? } \square \text { no, l've never smoked } & \square \text { no, but formerly I did } \square \text { yes } \\ \text { What do (or did) you smoke? (more than one answer possible) } & \square \text { cigarettes } & \square \text { cigars } \\ & \square \text { hand-rolled cigarettes } \square \text { pipe }\end{array}$

6 Do you have any special eating habits?

$\square$ no $\square$ macrobiotic $\square$ vegetarian $\square$ anthroposophic $\square$ vegan

$\square$ 7th day adventist other, namely

If so, since when? Since 19

7 How many days on average per week do you eat meat?

$\square 0$ days $\square 1$ day $\square 2$ days $\square 3$ days $\square 4$ days $\square 5$ days $\square 6$ days $\square 7$ days per week In case you never eat meat or only eat it once a week, since when have you been doing this? Since $19 \square \square$

8 Do (or did) you have any type of cancer? $\square$ no (go on to question 10) $\square$ yes

\begin{tabular}{|c|c|c|c|c|}
\hline If so, what type(s)? & $\begin{array}{l}\square \text { stomach } \\
\square \text { esophagus } \\
\square \text { large bowel } \\
\square \text { prostate }\end{array}$ & $\begin{array}{l}\square \text { bladder } \\
\square \text { skin } \\
\square \text { brains } \\
\square \text { testis }\end{array}$ & $\begin{array}{l}\square \text { kidney } \\
\square \text { leukemia } \\
\square \text { Hodgkin's disease } \\
\square \text { bone }\end{array}$ & $\begin{array}{l}\square \text { lung } \\
\square \text { oral cavity } \\
\square \text { non-Hodgkin lymphoma } \\
\square \text { liver }\end{array}$ \\
\hline
\end{tabular}

口 other, namely

\section{When was this discovered tor the first time? $\square 1986 \quad \square 1984 \quad \square 1982$}

$\square 1985 \square 1983 \square$ betore 1982, namely in $19 \square \square$

10 Has a physician ever diagnosed one of the following conditions and what was your age at that time?

(Put a mark in front of the condition and mark your age behind it)

\begin{tabular}{|c|c|c|c|c|c|c|c|c|c|c|}
\hline \multicolumn{3}{|c|}{ younger than 30} & \multirow{2}{*}{$\begin{array}{c}30-34 \\
\square\end{array}$} & \multirow{2}{*}{$\begin{array}{c}35-39 \\
\square\end{array}$} & \multirow{2}{*}{$\begin{array}{c}40-44 \\
\square\end{array}$} & \multirow{2}{*}{$\begin{array}{c}45-49 \\
\square\end{array}$} & \multirow{2}{*}{$\begin{array}{c}50-54 \\
\square\end{array}$} & \multirow{2}{*}{$\begin{array}{c}55-59 \\
\square\end{array}$} & \multirow{2}{*}{$\begin{array}{c}60-64 \\
\square\end{array}$} & \multirow{2}{*}{$\begin{array}{r}65-69 \\
\square\end{array}$} \\
\hline$\square$ & asthma & 口 & & & & & & & & \\
\hline$\square$ & chronic bronchitis & ロ & ロ & $\square$ & 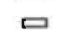 & 口 & 口 & 口 & 口 & 口 \\
\hline$\square$ & diabetes & ロ & ロ & $\square$ & $\sqsubset$ & ロ & 口 & 口 & 口 & 口 \\
\hline 口 & high blood pressure & 口 & 口 & 口 & 口 & 口 & ص & 口 & 口 & 口 \\
\hline$\hookrightarrow$ & heart attack & ロ & $\varpi$ & ص & ص & ロ & 口 & ロ & 口 & 口 \\
\hline ص & angina pectoris & ص & ロ & 口 & ロ & ロ & ロ & 口 & $\square$ & ロ \\
\hline 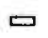 & gall-stones; & $\sqsubset$ & $\square$ & $\square$ & ص & $\square$ & $\square$ & $\square$ & 口 & ロ \\
\hline ص & kidney stones & 口 & 口 & ص & ロ & 口 & 口 & ロ & ロ & 口 \\
\hline$\square$ & thrombosis (in the leg) & $\curvearrowleft$ & 口 & $\square$ & 口 & 口 & $\square$ & $\sqsubset$ & $\square$ & 口 \\
\hline$\square$ & stroke & 口 & 口 & 口 & 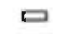 & 口 & $\square$ & 口 & 口 & 口 \\
\hline$\square$ & fuberculosis & ロ & $\square$ & $\square$ & $\square$ & 口 & $\square$ & $\square$ & $\square$ & 口 \\
\hline ש & gastric ulcer/bleeding & 口 & ロ & 口 & 口 & ロ & 口 & ロ & 口 & ロ \\
\hline$\square$ & chronic bowel irritation & ᄃ & 口 & $\square$ & 口 & $\square$ & $\square$ & 口 & $\square$ & 口 \\
\hline 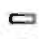 & polyps in bowel & 口 & ロ & 口 & 口 & 口 & 口 & 口 & 口 & ロ \\
\hline$=$ & hepatitis & ص & 口 & 口 & च & 口 & 口 & ロ & 口 & ロ \\
\hline 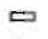 & rheurnatoid arthritis & ロ & 口 & 口 & 口 & $\square$ & 口 & 口 & $\square$ & 口 \\
\hline \multicolumn{11}{|c|}{ Have you ever had surgery on your gall-bladder or for a gastric ulcer, what was your age at that time? } \\
\hline$\square$ & gall-bladder & ロ & ロ & 口 & 己 & $\square$ & 口 & ロ & $\square$ & 口 \\
\hline$\square$ & stomach & 口 & 口 & 口 & 口 & 口 & 口 & ص & 口 & 口 \\
\hline
\end{tabular}


Please answer these questions as indicated in the example on the explanation form, preferably with pencil.

If this is not possible, you may use a blue or black pen. The answering possibilities have a white background.

(c) TNO/RL august 1988

1 Here your date of birth is printed, as given by you or your municipality:

Is this date correct? $\square$ yes (go on to question 2) $\square$ no

If not, are you the the person whose name and address are printed on the envelope?

$\square$ yes $\rightarrow$ If so, what is your correct date of birth? $\square \square-\square \square-19 \square \square$

$\square$ no $\rightarrow$ If so, could you give this letter to the addressee?

2 In what municipality were you born? (country)

\begin{tabular}{|lll}
\hline 3 What is your marital status? $\square$ never married & $\square$ divorced \\
& $\square$ married & $\square$ widowed
\end{tabular}

4 Do (or did) you have twin brother? $\square$ no $\square$ yes

If so, is his first initial the same as yours? $\square$ no $\square$ yes

5 Do you smoke currently? 口 no, l've never smoked

What do (or did) you smoke? (more than one answer possible)

$$
\begin{array}{ll}
\square \text { no, but formerly I did } & \square \text { yes } \\
\square \text { cigarettes } & \square \text { cigars } \\
\square \text { hand-rolled cigarettes } & \square \text { pipe }
\end{array}
$$

\section{Do you have any special eating habits?}
$\square$ no
ㅁ. macrobiotic
$\square$ vegetarian
$\square$ anthroposophic $\square$ vegan
$\square$ 7th day adventist
$\square$ other, namely
(don't mention diets)
If so, since when? Since 19

7 How many days on average per week do you eat meat?

$\square 0$ days $\square 1$ day $\square 2$ days $\square 3$ days $\square 4$ days $\square 5$ days $\square 6$ days $\square 7$ days per week

In case you never eat meat or only eat It once a week, since when have you been doing this? Since $19 \square \square$

8 Do (or did) you have any type of cancer?

If so, what type(s)? $\begin{aligned} & \square \text { stomach } \\ & \\ & \quad \square \text { esophagus } \\ & \square \text { large bowel } \\ & \square \text { breast } \\ & \square \text { uterus }\end{aligned}$

$\square$ no (go on to question 10) $\square$ yes

$\square$ bladder

$\square$ skin

$\square$ kidney

$\square$ leukemia.

$\square$ lung

$\square$ brains.

$\square$ Hodgkin's disease

$\square$ oral cavity

$\square$ ovary

$\square$ bone

口non-Hodgkin lymphoma

$\square$ uterus

$\square$ cervix

$\square$ other, namely

\begin{tabular}{|l|llll}
\hline 9 When was this discovered for the first time? & $\square 1986$ & $\square 1984$ & $\square$ 1982 \\
& $\square 1985$ & $\square 1983$ & $\square$ befóre 1982, namely in $19 \square$ \\
\hline
\end{tabular}

10 Has a physiclan ever diagnosed one of the following conditions, and what was your age at that tlme?

(Put a mark in front of the condition and mark your age behind it)

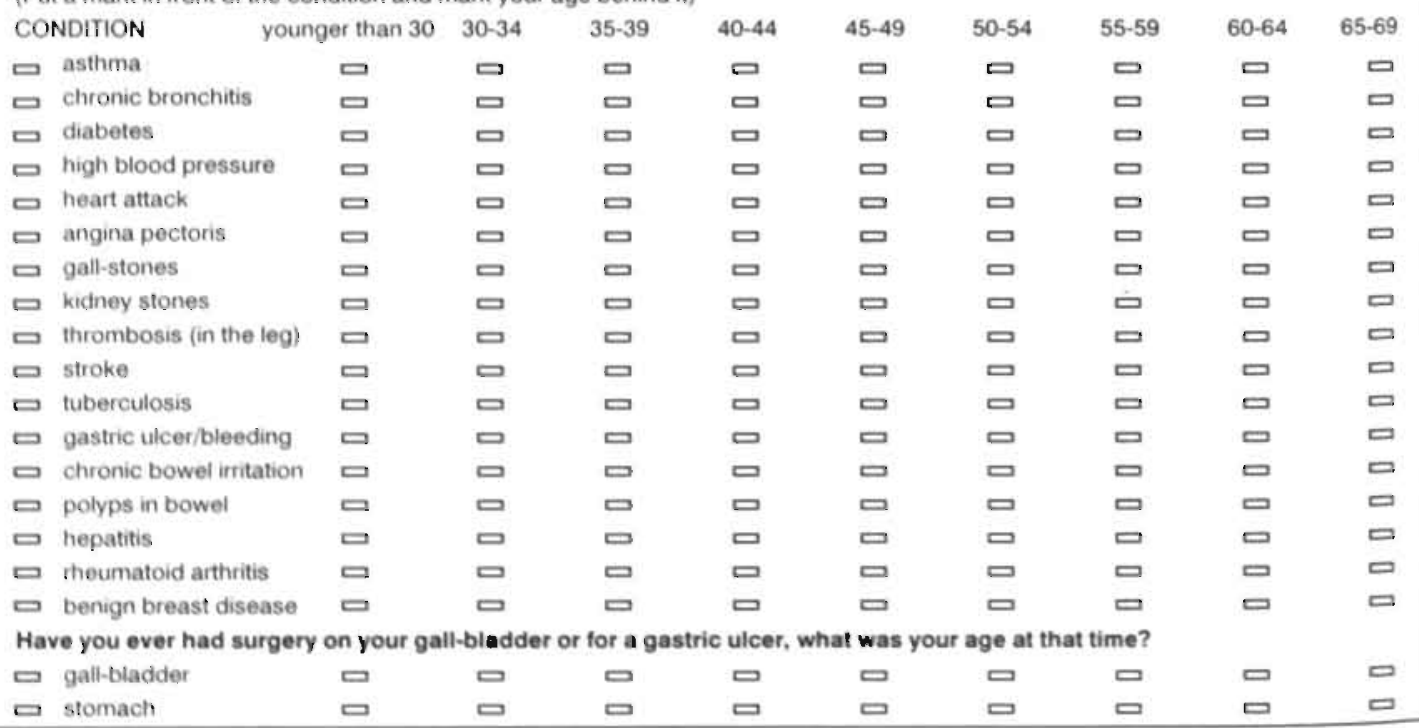


The following questions concern your EATING AND DRINKING habits of the past year, in case you don't know an answer because you don't prepare meals yoursell, please ask the one who does. Remember that the questions concern the things you eat and drink. If you don't understand some questions, please ask your partner, friends or childeren to help you.

Sometimes your are requested to specify a certain brand. The reason for this is that different brands may difler in compostion; the information is not used for commercial purposes. When the distinction "in summer" and "in winter" is used, approximate periods are meant, not strictly periods of three months.

11 How many eggs do you eat per week?
Do you drink coffee?
Do you take sugar in your coffee?

12 How many times per week do you use a hot meal? $\square \square$ times per week Do you prepare the hot meals yourseln? $\square$ no, seldom or never $\square$ yes

How often did you have these products for your hot meal during the past year?

bread instead of a hot meal

Chinese or Indonesian food

- Italian tood (e.g. pasta. pizza)

- soup as main course

fish

egg instead of meat

cheese instead of meat

meat or chicken

. pulses (e.g. white or kidney beans, lentils, marrowiats)

. soybean products (e.g. tofu, tempeh)

white rice (not in Chinese food)

brown rice

millet, buckwheat, wheat, barley,

oats and other cereals

- trench fried potatoes

. mayonnaise

potatoes (boiled, fried, mashed or

In a mixed dish)

raw vegetables and boiled

vegetables: in winter

in summer

only raw vegetables without boiled

vegetables: in winter

in summer

. only boiled vegetables: in winter

in summer

\begin{tabular}{|c|c|c|c|c|c|c|c|}
\hline never or & $1 x$ & $2-3 x$ & $1 x$ & $2-3 x$ & $4-5 x$ & $6-7 x$ & how much dida yout eat? \\
\hline less than & per & per & per & per & per & per & \\
\hline 1x/month & mo. & mo. & $\begin{array}{l}\text { week } \\
\square\end{array}$ & $\begin{array}{l}\text { week } \\
\square\end{array}$ & $\begin{array}{l}\text { week } \\
\square\end{array}$ & week & shices \\
\hline ص & 口 & ص & 口 & $\square$ & 口 & 口 & serving spoons \\
\hline 口 & $\square$ & 口 & 口 & $\square$ & $\square$ & 口 & serving spoons \\
\hline 口 & $\square$ & $\square$ & $\square$ & 口 & $\square$ & 口 & serving spoons \\
\hline 口 & ص & च & च & ロ & 口 & 口 & \\
\hline 口 & ロ & $\square$ & 口 & $\square$ & 口 & $\square$ & \\
\hline 口 & $\square$ & 口 & $\square$ & $\square$ & 口 & 口 & \\
\hline • & ロ & 口 & 口 & 口 & $\square$ & 口 & \\
\hline$\square$ & 口 & $\square$ & $\square$ & $\square$ & $\square$ & $\square$ & serving spoons \\
\hline$\square$ & ロ & $\square$ & $\square$ & $\square$ & $\square$ & $\square$ & tablespoons \\
\hline 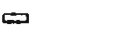 & 口 & घ & $\sqsubset$ & 口 & $\square$ & 口 & serving spoons \\
\hline$\square$ & $\varnothing$ & ఐ & ص & $\square$ & $\curvearrowleft$ & 口 & serving spoons \\
\hline$\square$ & $\square$ & $\square$ & $\square$ & ص & $\square$ & $\square$ & serving spoons \\
\hline$\square$ & $\square$ & $\bullet$ & $\square$ & $\varnothing$ & $\Xi$ & ص & \\
\hline$\infty$ & (두 & $\square$ & 口 & $\curvearrowleft$ & $\square$ & $\square$ & tablespoons \\
\hline 口 & C & $\square$ & $\square$ & $\infty$ & $\square$ & $\square$ & pieces the size \\
\hline$\square$ & ए & $\square$ & $\sqsubset$ & $\infty$ & $\square$ & $\square$ & of an eggl \\
\hline$\square$ & ᄃ & $\varpi$ & $\square$ & a & $\Leftrightarrow$ & $\varphi$ & \\
\hline$\square$ & $\varpi$ & $\square$ & $\square$ & 口 & $\square$ & $\square$ & \\
\hline$\infty$ & $\varpi$ & $\square$ & $\boxminus$ & ص & $\square$ & $\square$ & \\
\hline$\square$ & 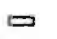 & ص & $\square$ & 口 & ص & 口 & \\
\hline$\square$ & $\square$ & $\square$ & 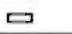 & $\square$ & ロ & $\varpi$ & \\
\hline
\end{tabular}

(0) TNO/AL august 1986 
38 Have you ever chewed tobacco? $\square$ no $\square$ yes, during $\square$ years Have you ever sniffed tobacco? $\square$ no $\square$ yes, during $\square \square$ years

39 Did your father or mother smoke when you were living with them?

$\begin{array}{ll}\square \text { no } & \square \text { mother only } \\ \square \text { both } & \square \text { father only }\end{array}$

Does or did your partner smoke in your company? no What does or did your partner smoke in your company? Are or were you exposed to cigarette smoke at work? $\square$ cigarettes $\square$ father only How many hours a day on average are you exposed to cigarette smoke? $\quad \square \quad \square$ hours (remember home, car, work, canteens etc.)

\section{EDUCATION AND OCCUPATION}

40 Which of the following schools did you attend and which did your partner attend?

me pertner

$\square$ primary school

$\square$ lower vocational education (e.g. technical, domestic science school)

$\square$ junior high school

$\square$ senior high school

$\square$ higher vocational education

口 university

o other, e.g. specific part-time education, namely

口 other, e.g. specific part-time education, namely

41 Have you ever had paid employment? $\square$ yes, wage-earning $\square$ no, but did have an unpaid job $\square$ yes, sell-employed $\square$ no (go on to question 43 )

What have been your occupations In your llfetime? At what companies or instltutions did you work, what was belng produced? What was your job and for how long dld you work there? Fill in this information in the space below, as in the example on the first line. If you are/were self-employed, fill in the type of company and your exact job only.

name of company of institution?

Johuson lac

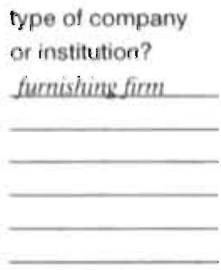

what was being produced your exact at your department job? sofar etc.

$\square$ na $\square$ yes

42 Are you currently employed?

If not, which situation applies to you?

$\begin{array}{ll}\square & \text { temporarily unemployed } \\ \square & \text { retired } \\ \square \quad \text { ather, namely }\end{array}$

$\square$ disablẹ

$\square$ exclusively homemaker (only included in questionnaire for women)

C TNO/RL. august 1986 
43 How do you consider your health in general? $\square$ excellent $\square$ good $\square$ reasonable $\square$ poor $\square$ bad How many hours of sleep do you usually get per day? in the day-time and at night) $\quad \square$ hours

44. What surgical operations have you had and at what age? (name the last three)

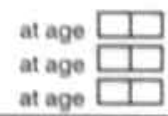

45. Have you ever had an X-ray photograph taken of your:

$\begin{array}{llll}\text { stomach } & \square \text { no } & \square \text { yes if so, how often? } & \square \text { times } \\ \text { boweis } & \square \text { no } & \square \text { yes if so, how often? } & \square \text { times } \\ \text { lung/chest } & \square \text { no } & \square \text { yes if so, how often? } & \square \text { times } \\ \text { neck or shoulder } & \square \text { no } & \square \text { yes if so, how often? } \\ \text { back } & \square \text { no } & \square \text { yes if so, how often? }\end{array}$

other, namely — If so, how often? $\square \square$ times.

Have you ever had radiotherapy? no $\square$ yes if so, for what disease?

46. Please list below what medicine(s) you have used longer than six months, for what condition(s) and in what periods)? name of medicine for what condition

\begin{tabular}{|c|c|}
\hline from & to \\
\hline from 19 & to 19 \\
\hline from 19 & to 18 \\
\hline from 19 & to 19 \\
\hline om 19 & to 18 \\
\hline
\end{tabular}

47 What is your blood type? $\square$ don't know $\square 0 \square \square A \square$

What is your rhesus factor? $\square$ don't know $\square$ positive $\square$ negative

48 Did any of your parents, brothers or sisters have cancer? $\square$ no $\square$ yes If so, please fill this in in the space below, as in the example.

what relation type of cancer

sister

How many brothers and sisters do (did) you have?

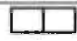
biothers and $\square$ sisters.

age at diagnosis if alive, give current age

at age 512 at age $\square$ at age $\square$ at age $\mathrm{T}$ 5 [. 1 years oid $\square$ years ald $\square$ years old $\square$ years old if dead, give age at death at age at age at age at age In what year were your father and mother born? In case they have died, in what years did they die, and what was the cause of death? father: born in $19 \square$ (died in $19 \square$; cause of death: mother: born in $19 \square \square$ (died in $19 \square \square$; cause of death: 
49 How often do you usually have a bowel movement? $\square$ more than 2 times per day $\square_{1-2}$ times per day $\square 1$ time per day $\square$ 1 time per 2 days $\square 2$ times per week or less Do you ever suffer from obstipation? $\square$ never $\square$ seidom $\square$ sometimes $\square$ often $\square$ very often

\section{OTHEA QUESTIONS}

50 How tall are you?

How much do you weigh?

What size do you wear? centimeters

kilograms

shirt:
What was your weight at age 20 ? $\square \square \square$ kilograms pants: $\square$

51. Please fill in below the last four residences where you have liveci.

residence

province

residence

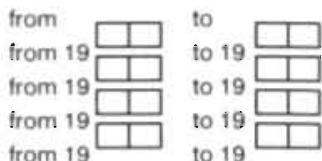

to 19

What was your residence during the winter from 1944-1945?

52 Was your father employed during the crisis (1932-1940)?

$\square$ no $\square$ yes, he was employed in that period from 19

$1019 \square$

53 How many minutes do you spend on average per day walking or cycling to your work, to go shopping or to take out your dog? $\square \square$ minutes per day

How many hours of your leisure time do you spend on average per week on the following acivities?
gardening
$\square$ I never do this
$\square$ less than 1 hour
ㄷ $1-2$ hours
$\square$ more than 2 hours
cycling, walking
$\square$ I never do this
$\square$ less than 1 hour
口 1.2 hours
$\square$ more than 2 hours
sports, gymnastics
$\square$ I never do this
$\square$ less than 1 hour
口 $t-2$ hours
more than 2 hours

In case you play sports, what spoirts do you play?

54 In case you formerly played sports, piease indicate in the table below: what sport(s), was it in a competition system, how many hours per week did you spend on each sport (exercise included) and in what period?

type of sport

$\begin{array}{lll}\text { competition how many hours per week } & \text { from } \\ \square \text { no } \square \text { yes } \square \text { hours per week } & \text { from 19 } \square \\ \square \text { no } \square \text { yes } \square \square \text { hours per week } & \text { from 19 } \square \square \\ \square \text { no } \square \text { yes } \square 1 & \text { hours per week } & \text { from } 19 \square \square\end{array}$

to

55. This question concems leisure interests you have (had), e.g. fishing, woodcarving, dark room activities, painting. On what leisure intereats do you spend a faitr amount of time?

56 Do you keep pets inside your home? (e.g. birds, hamster, dog) $\square$ no $\square$ yes If so, what animals? 
43 How do you consider your health in general? $\square$ excellent $\square$ good $\square$ reasonable $\square$ poor $\square$ bad How many hours of sleep do you usually get per day? (in the day-time and at night) $\square \square$ hours

44 What surgical operations have you had and at what age? (name the last three)

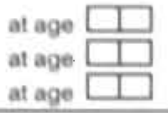

45. Have you ever had an X-ray photograph taken of your:
$\begin{array}{ll}\text { stomach } & \square \text { no } \square \text { yes if so, how often? } \\ \text { bowels } & \square \text { no } \square \text { yes if so, how often? } \\ \text { lung/chest } & \square \text { no } \square \text { yes it so, how often? } \\ \text { neck or shoulder } & \square \text { no } \square \text { yes if so, how often? } \\ \text { back } & \square \text { no } \square \text { yes it so, how often? } \\ \text { breasts } & \square \text { no } \square \text { yes if so, how often? }\end{array}$

other, namely

Have you ever had radiotherapy? $\square$ no. $\square$ yes if so, for what disease?

If so, how often? $\square$ times

46. Please list below what medicine(s) you have used longer than six months, for what condition(s) and in what periods)? name of medicine for what condition

from $\begin{aligned} & \text { to } \\ & \text { from } 19 \square \square \\ & \text { from } 19 \square \square \\ & \text { to } 19 \\ & \text { from } 19 \square \square \\ & \text { from } 19 \square \square \\ & \text { to } 19 \square \square \\ & \text { to } 19 \square \square\end{aligned}$

47 At what age did you have your first menstrual period? at age $\square$

Have you ever used oral contraceptives? $\square$ no $\square$ yes

If so, at what age did you start using oral contraceptives? at age $[\square$

Are you still using oral contraceptives? $\square$ yes $\square$ no If not, when did you stop? at age $\square[]$

How many years have you used oral contraceptives, excluding the years you stopped? $\square$, years

At what age did your menopause start oft? at age $\square$

How did your menopause start off? $\square$ naturally $\square$ after surgery $\square$ with medication

Have you ever used hormones because of a discomfort during/after the menopause? $\square$ no $\square$ yes $\square$ don'tknow

If so, for how long did you use these hormones? fiom ig i

48 Have you ever had a Pap smear? $\square$ no $\square$ yos if so, when was the last time? in $19 \square$

Were you ever screened for breast cancer? $\square$ na $\square$ yes

If so, when was the last time? in i9 $\square$

49 In case you are (or were) married, in what year were you married for the first time? in $19 \square \square$

Did you have childeren? $\square$ no $\square$ yes if so, how many $[\perp]$ (also mention stillbirths, don't mention miscarriages)

If so, in what year was your first child born? in $19 \square$ 
50 Did any of your parents, brothers or sisters have cancer? $\square$ no $\square$ yes if so, please fill this in in the space below, as in the example.

what relation

type of cancer

sister

lireast

How many brothers and sisters do (did) you have? brothers and

\section{age at diagnosis}

at age $5 \mathrm{SL}$

at age $\frac{1}{\square}$

at age $\square$

at age $\square$

In what year were your father and mother born?

In case they have died, in what years did they die, and what was the cause of death?

father: born in $19 \square$ (died in $19 \square$; cause of death:

mother: born in $19 \square \square$ (died in $19 \square \square$; cause of death:

\section{OTHER QUESTIONS}

51 How tall are you?

How much do you weigh?

What size do you wear?

\section{$\square$ centimeters \\ $\square$ kilograms} blouse: $\square$
What was your wolght at age 20? $\square \square$ kilograms skirt: $\square$

52 Please IIII In below the last four residences where you have lived.

residence

province

residence

if alive, give

current age

$5[2$ years old

I years old

$\square$ years old

$\square$ years oid if dead, give age at death at age $\square$ at age at age at age resconger

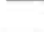

What was your residence during the winter from $1944-1945 ?$ from

from $19 \square$

from $19 \square$

from $19 \square$

from $19 \square \square$

province (country)

53 Was your father employed during the crisls (1932-1940)?

CJ no $[J$ yes, he was employed in that period from 19

to $19 \square \square$

54 How many minutes do you spend on avr rage per day walking or cycling to your work, to go shopping or to take out your dog? LI minutes per day

How many hours of your leisure time do you spend on average per week on the following acivities?

$\begin{array}{llll}\text { gardening } & \square \text { I never do this } \square \text { less than } 1 \text { hour } \square 1-2 \text { hours } & \square \text { more than } 2 \text { hours } \\ \text { cycling, walking } & \square \text { I never do this } \square \text { less than } 1 \text { hour } \square 1-2 \text { hours } & \square \text { more than } 2 \text { hours } \\ \text { sports, gymastics } \square \text { I never do this } \square \text { less than } 1 \text { hour } \square 1-2 \text { hours } & \square \text { more than } 2 \text { hours }\end{array}$

In case you play sports, what sports do you play?

55 In case you formerly played sports, please indicate in the table below: what sport(s), was it in a competition system, how many hours per week did you spend on each sport (exercise included) and in what period?

type of sport

competition how many hours per week
$\square$ no $\square$ yes $\square \square$ hours per week
$\square$ no $\square$ yes $\square$ nours per week
$\sigma$ no $\square$ yes $\square$ hours per week

$\begin{array}{ll}\text { from } & \text { to } \\ \text { from } 19 \square \square & \text { to } 19 \square \square \\ \text { from } 19 \square \square & \text { to } 19 \square \square \\ \text { from } 19 \square \square & \text { to } 19 \square \square\end{array}$




\section{About the authors}

Sandra Goldbohm was born in Noordwijk on October 21, 1951. After graduating from Gymnasium- $\beta$ at the Kennenner Lyceum in Overveen in 1969, she spent a year in Switzerland to learn French. In 1970, she entered the Agricultural University in Wageningen to study Human Nutrition and graduated (cum laude) in 1978 (majors: nutrition and epidemiology). During this study, she spent six months as "stageaire" with the Secretary of the Scientific Conmmittee for Food of the EC in Brussels. From 1978 to 1982 she worked in the Department of Environmental and Tropical Health of the same university and was engaged in clata analysis and publication of a monograph on the Kaunas Rotterdam Intervention Study on cardjovascular disease and in teaching epidemiology. At the same time, she was a member of a governmentall committee to design a study on the efficacy of the "Moerman" therapy. She was also involved in founding of the WEON, predecessor of the Netherlands Epidemiological Society, of which she later became board member. From 1982 to 1984, she was awarded a research fellowship by the Dutch Cancer Society. The first year was spent to obtain a MSc degree in epidemiology at the Harvard School of Public Health (Boston, USA), whereas during the second year she wrote a grant proposal for the cohort study described in this thesis and started with the pilot study. Since 1983, she runs the cohort study, while stationed at the department of Nutrition of the TNO Toxicology and Nutrition Institute in Zeist. She is married to Edward Bausch and since 1984 they both combine their work with the care of their three sons of 8,6 and 3 years old.

Piet van den Brandt was born on October 21, 1957 in Vljinen, the Netherlands. After graduating from Gymnasium- $\beta$ at the Prof. ter Veenlyceum in Emmeloord in 1976, he started to study Human Nutrition at the Agricultural University in Wageningen. In 1980 he obtained his BSc degree (cum laude) and in 1983 his MSc degree in Human Nutrition. The MSc program was focused on nutrition (in particular nutritional epidemiology), toxicology and statistics. Part of the MSc program was spent at the Dunn Nutritional Laboratory (Cambridge, UK). In 1983-1985 he was awarded a research fellowship by the Dutch Cancer Society. During part of this period he worked at the Department of Epidemiology, University of Limburg in Maastricht and the Department of Nutrition, TNO Toxicology and Nutrition Institute in Zeist. In addition, he obtained a Master of Science degree in Epidemiology in 1984 at the Harvard Scbool of Public Health (Boston, USA). Research during this fellowship was concentrated on the design and pilot study of a prospective colort study on diet and cancer. In 1985 he became research associate at the Department of Epidemiology of the University of Limburg. Since 1986, he is assistant professor at this Department, first, in the Faculty of Medicine and from 1991 onwards in the Faculty of Health Sciences. His research activities focus on the prospective cohort study on diet and cancer described in this thesis. From 1987 onwards, be also works as Consultant on Epidemiology at the Comprehensive Cancer Centre Limburg in Maastricht. 
P.A. van den Brandt \& R.A. Bausch-Goldbohm

\section{A prospective cohort study on diet and cancer in the Netherlands}

This thesis describes a prospective cohort study on dietary habits and the risk of cancer, conducted in the Netherlands since 1986 among more than 120,000 men and women. Methodological issues, such as the design of the study and the development and validation of methods, form a substantial part of the thesis. First results after 3.3 years of follow-up are also presented. Chapters are devoted to the associations between the dietary intake of fat and the risk of breast cancer, and between consumption of alcoholic beverages and meat and the risk of cancer of the large bowel. Furthermore, the risk of bowel cancer after gallbladder surgery is being assessed as well as the association between selenium content of toenail elippings and the risk of cancer of the lung, gastrointestinal tract and breast. 